\title{
RSSDI-ESI Clinical Practice Recommendations for the Management of Type 2 Diabetes Mellitus 2020
}

Rajeev Chawla', S. V. Madhu², B. M. Makkar³, Sujoy Ghosh", Banshi Saboo ${ }^{5}$, Sanjay Kalra ${ }^{6}$, On behalf of the RSSDI-ESI Consensus Group*

${ }^{1}$ North Delhi Diabetes Centre, Rohini, New Delhi, ${ }^{2}$ Centre for Diabetes, Endocrinology and Metabolism, UCMS-GTB Hospital, ${ }^{3}$ Dr. Makkar's Diabetes and Obesity Centre, Paschim Vihar, New Delhi, ${ }^{4}$ Department of Endocrinology and Metabolism, Institute of Post Graduate Medical Education and Research, Kolkata, West Bengal, ${ }^{5}$ DiaCare - A Complete Diabetes Care Centre, Ahmedabad, Gujarat, ${ }^{6}$ Department of Endocrinology, Bharti Hospital, Karnal, Haryana, India

\section{*RSSDI-ESI Consensus Group}

\section{Diagnosis of Diabetes}

Prevention (Including Screening and Early Detection)

Treatment 1: Medical Nutrition Therapy and Lifestyle Modification

Treatment 2: Oral Antidiabetic Agents

Treatment 3: Injectables

Treatment 4: Individualizing Therapy

Post Prandial Hyperglycaemia

Acute Metabolic Complications

Hypoglycaemia

Chronic Complications 1: Retinopathy, Neuropathy, Diabetic Kidney Disease

Chronic Complications 2: Diabetic Foot and Peripheral Arterial Disease
Coordinators: S. R. Aravind, Ganapathi Bantwal Members: A. H. Zargar Nihal Thomas, Alok Kanugo Coordinators: S. V. Madhu, R. M. Anjana Members: A. Ramachandran, Eesh Bhatia, Amitesh Agarwal Coordinators: Anoop Misra, Naval Vikram, Members: Parminder Singh, Sambit Das, Vageesh Ayyar Coordinators: Shashank Joshi, Krishna Seshadri Members: Vijay Panikar, Sameer Agarwal, V. Sringesh Coordinators: A. K. Das, Sanjay Agarwal Members: Ajay Kumar, Sunil Jain, Sujit Jha Coordinators: Banshi Saboo, A. K. Singh Members: Muralidharan, Santosh Singh, Rajesh Khadgawat Coordinators: Hari Kumar, Kaushik Pandit Members: S. K. Sharma, Mathew John, Pradip Mukhopadhyay Coordinators: Jubin Jacob, Anil Bhansali

Members: Sushil Jindal, Kamlakar Tripathi, Narsingh Verma Coordinators: Anand Moses, Jamal Ahmed Members: D. C. Sharma, G. Vijaykumar, Dheeraj Kapoor Coordinators: Rajeev Chawla, Rakesh Sahay Members: Shalini Jaggi, R. K. Lalwani, Hitesh Punyani Coordinators: S. K. Singh, Vijay Vishwanathan Members: Ashu Rastogi, Ghanshyam Goyal, Ashraf Ganie 


\begin{tabular}{ll}
\hline Diabetes and CVD & Coordinators: V. Mohan, Arvind Gupta \\
Obesity and Type 2 Diabetes Mellitus & Members: Shaileja Kale, Sudhir Bhandari, R. V. Jayakumar \\
Coordinators: B. M. Makkar, Neeta Deshpande, & Members: C. L Nawal, K. A. V. Subramanian, Rucha Mehta \\
Infections and Vaccinations & Coordinators: Subhankar Chowdhary, Jayant Panda \\
Sexual Dysfunction & Members: Pramod Gandhi, J. K. Sharma, Vinay Dhandhania \\
Clycaemic Monitoring & Members: Mithun Bhartiya, Urman Dhruv, Premlata vartakavi \\
Technologies & Coordinators: Siddhartha Das, A. G. Unnikrishnan \\
Special Situations & Members: Rajiv Kovil, Alok Sachan, Girish Varma \\
Fasting and diabetes & Coordinators: Jothydev, Ambrish Mithal \\
Education & Members: K. M. Prasanna Kumar, Manoj Chawla, Ameya Joshi \\
Psychosocial Issues & Coordinators: Nikhil Tandon, Padma Menon \\
Complementary and Alternate Therapies & Members: Sujoy Ghosh, Nalini Shah, Usha Sriram, Rajesh Rajput \\
& Coordinators: Sarita Bajaj, Manas Barua \\
& Members: M. K. Garg, Sachin Chhitawar, Hemant Thacker \\
& Coordinators: Vasanth Kumar, Sunil Gupta \\
& Members: Meena Chhabra, Amit Gupta, G. D. Ramchandani \\
& Coordinators: H. B. Chandalia, Sanjay Kalra \\
Members: G. R. Sridhar, Rishi Shukla, Salam Ranabir
\end{tabular}

\section{Diagnosis OF Diabetes}

\section{RECOMMENDATIONS}

\section{Recommended Care}

Prediabetes can be diagnosed with any of the following criteria

- Impaired fasting glucose (IFG): FPG $100 \mathrm{mg} / \mathrm{dL}$ to $125 \mathrm{mg} / \mathrm{dL}$ or

- Impaired glucose tolerance (IGT): 2-h plasma glucose (2-h PG) during 75-g OGTT $140 \mathrm{mg} / \mathrm{dL}$ to $199 \mathrm{mg} / \mathrm{dL}$ or

- $\mathrm{HbA} 1 \mathrm{c} \geq 5.7 \%-6.4 \%$

Diabetes can be diagnosed with any of the following criteria:

- $\mathrm{FPG} \geq 126 \mathrm{mg} / \mathrm{dL} *$ or

- $\mathrm{FPG} \geq 126 \mathrm{mg} / \mathrm{dL}$ and/or $2-\mathrm{h} \mathrm{PG} \geq 200 \mathrm{mg} / \mathrm{dL}$ using 75 -g OGTT

- $\mathrm{HbA} 1 \mathrm{c} \geq 6.5 \% * *$ or

- Random plasma glucose $\geq 200 \mathrm{mg} / \mathrm{dL}$ in the presence of classical diabetes symptoms

Asymptomatic individuals with a single abnormal test should have the test repeated to confirm the diagnosis unless the result is unequivocally abnormal.

\section{Limited Care}

Diabetes can be diagnosed with any of the following criteria:

- $\mathrm{FPG} \geq 126 \mathrm{mg} / \mathrm{dL} *$ or

- $\mathrm{FPG} \geq 126 \mathrm{mg} / \mathrm{dL}$ and/or 2 -h plasma glucose $\geq 200 \mathrm{mg} / \mathrm{dL}$ using 75 -g OGTT or

- Random plasma glucose $\geq 200 \mathrm{mg} / \mathrm{dL}$ in the presence of classical diabetes symptoms

Asymptomatic individuals with a single abnormal test should have the test repeated to confirm the diagnosis unless the result is unequivocally abnormal

\section{NOTE}

- Estimation of HbAlc should be performed using NGSP standardized method.

- Capillary glucose estimation methods are not recommended for diagnosis

- Venous plasma is used for estimation of glucose
- Plasma must be separated soon after collection because the blood glucose levels drop by $5 \%-8 \%$ hourly if whole blood is stored at room temperature.

For more details on glucose estimation visit: http://www.ncbi. nlm.nih.gov/books/NBK248/ 
*FPG is defined as glucose estimated after no caloric intake for at least 8-12 hours.

**Using a method that is National Glycohaemoglobin Standardization Program (NGSP) certified. For more on HbAlc and NGSP, please visit http://www.ngsp.org/index. asp

\section{BACKGROUND}

The diagnostic criteria of diabetes have been constantly evolving. Both type 1 and type 2 Diabetes mellitus (DM) are diagnosed based on the plasma glucose criteria, either the fasting plasma glucose (FPG) levels or the 2-h plasma post-prandial glucose (2-h PPG) levels during a 75 -g oral glucose tolerance test (OGTT), or the newer glycosylated haemoglobin $(\mathrm{HbA} 1 \mathrm{c})$ criteria which reflects the average plasma glucose concentration over the previous 8-12 weeks. ${ }^{[1,2]}$ The International Expert Committee Report recommend a cut-point of $\geq 6.5 \%$ for $\mathrm{HbA} 1 \mathrm{c}$ for diagnosing diabetes as an alternative to fasting plasma glucose (FPG $\geq 7.0 \mathrm{mmol} / \mathrm{L}){ }^{[3]} \mathrm{HbA} 1 \mathrm{c}$ testing has some substantial advantages over FPG and OGTT, such as convenience, pre-analytical stability, and less day-to-day fluctuations due to stress and illness. ${ }^{[3]}$ Additionally, HbA1c has been recognized as marker to assess secondary vascular complications due to metabolic derailments in susceptible individuals. ${ }^{[2,4,5]}$ However, given ethnic differences in sensitivity and specificity of $\mathrm{HbA} 1 \mathrm{c}$ population-specific cut-offs might be necessary. ${ }^{[6,7]}$ Moreover, measuring $\mathrm{HbA} 1 \mathrm{c}$ is expensive as compared to FPG assessments and standardization of measurement techniques and laboratories are poorly practiced across the country. ${ }^{[8]}$ Also, in several countries including India, $\mathrm{HbA} 1 \mathrm{c}$ demonstrated inadequate predictive accuracy in the diagnosis of diabetes, there is no consensus on a suitable cut-off point of HbAlc for diagnosis of diabetes in this high-risk population. ${ }^{\left[{ }^{[9]}\right.}$ In lieu of this, the panel expressed concerns on using $\mathrm{HbA} 1 \mathrm{c}$ as sole criteria for diagnosis of diabetes particularly in resource constraint settings. Therefore, a combination of HbAlcand FPG would improve the identification of individuals with diabetes mellitus and prediabetes in limited resource settings like India.

\section{Considerations}

The decision about setting diagnostic thresholds values was based on the cost-effective strategies for diagnosing diabetes that were reviewed in Indian context.

\section{Rationale and Evidence}

\section{Glycosylated haemoglobin cut off for diagnosis of diabetes} in Indian patients

- The RSSDI expert panel suggests

- HbA1c $\geq 6.5 \%$ as optimal level for diagnosis of diabetes in Indian patients

- HbAlc cannot be used as 'sole' measurement for diagnosis of diabetes in Indian settings.

These recommendations are based on the Indian evidences

- A recent study conducted in Singapore residents of Chinese, Malay and Indian race to assess the performance of $\mathrm{HbAlc}$ as a screening test in Asian populations suggested that $\mathrm{HbA} 1 \mathrm{c}$ is an appropriate alternative to FPG as a first-step screening test, and a combination of $\mathrm{HbA} 1 \mathrm{c}$ with a cut-off of $\geq 6.1 \%$ and FPG level $\geq 100 \mathrm{mg} / \mathrm{dL}$ would improve detection in patients with diabetes. ${ }^{[6]}$

- A study to assess the diagnostic accuracy and optimal $\mathrm{HbA1c}$ cutoffs for diabetes and prediabetes among highrisk south Indians suggested that $\mathrm{HbA} 1 \mathrm{c} \geq 6.5 \%$ can be defined as a cut-off for diabetes and $\mathrm{HbAl} 1 \mathrm{c} \geq 5.9 \%$ is optimal for prediabetes diagnosis and value $<5.6 \%$ excludes prediabetes/diabetes status. ${ }^{[8]}$

- Data from a community based randomized cross sectional study in urban Chandigarh suggest that $\mathrm{HbAlc}$ cut point of $6.5 \%$ has optimal specificity of $88 \%$, while cut off point of $7.0 \%$ has sensitivity of $92 \%$ for diagnosis of diabetes. ${ }^{[10]}$

- The results of the Chennai Urban Rural Epidemiology Study (CURES) demonstrated $88.0 \%$ sensitivity and $87.9 \%$ specificity for detection of diabetes when $\mathrm{HbA1c}$ cut off point is $6.1 \%$ (based on 2-h post load plasma glucose) and $93.3 \%$ sensitivity and $92.3 \%$ specificity when $\mathrm{HbA} 1 \mathrm{c}$ cut off point is $6.4 \%$ (when diabetes was defined as $\mathrm{FPG} \geq 7.0 \mathrm{mmol} / \mathrm{l}) .{ }^{[11]}$

However, panel emphasized that $\mathrm{HbAlc}$ can be used in settings where an appropriate standardized method is available.

\section{IMPLEMENTATION}

Individuals should be educated on the advantages of early diagnosis and should be encouraged to participate in community screening programs for diagnosis. 


\section{Prevention}

\section{ReCOMmEndations}

\section{Recommended Care}

Screening and early detection

- Each health care service provider should have a program to detect people with undiagnosed diabetes.

- This decision should be based on the prevalence of undiagnosed diabetes and available support from health care system/service capable of effectively treating newly detected cases of diabetes.

- Opportunistic screening for undiagnosed diabetes and prediabetes is recommended. It should include:

- Individuals presenting to health care settings for unrelated illness

- Family members of diabetes patients

- Antenatal care

- Dental care

- Overweight children and adolescents at onset of puberty

- Wherever feasible, community screening may be done

- Detection programs should be usually based on a two-step approach:

- Step 1: Identify high-risk individuals using a risk assessment questionnaire

- Step 2: Glycaemic measure in high-risk individuals

- Where a random non-FPG level $\geq 100$ (or random capillary glucose $>110 \mathrm{mg} / \mathrm{dL}$ ) to $<200 \mathrm{mg} / \mathrm{dL}$ is detected, OGTT should be performed.

- Use of HbA1c as a sole diagnostic test for screening of diabetes/prediabetes is not recommended.

- Universal screening and diagnosis of gestational diabetes mellitus must be done to identify women at very high risk of future diabetes and cardiovascular diseases (CVD).

- People with high blood glucose during screening need further diagnostic testing to confirm diabetes while those with screen-negative for diabetes should be retested as advised by the physicians.

- Paramedical personnel such as nurses or other trained workers should be included in any basic diabetes care team.

Prediabetes

- People who are screen-positive for prediabetes (FPG=100-125 mg/dL or 2-h PG in the $75-\mathrm{g}$ OGTT=140-199 mg/dL or HbA1c=5.7\%-6.4\%) should be monitored for development of diabetes annually.

- Should be simultaneously screened and treated for modifiable risk factors for CVD such as hypertension, dyslipidaemia, smoking, and alcohol consumption.

- Screening strategies should be linked to health care system with capacity to provide advice on lifestyle modifications:

- May be aligned with ongoing support national programs available at community health centres

- Patients with IGT, IFG should be referred to these support programs.

- People with prediabetes should modify their lifestyle including:

- Attempts to lose $5 \%-10 \%$ of body weight if overweight or obese

- Participate in physical activity (e. g., walking) for at least 1-h daily if overweight or obese. and at least half hour daily if normal weight

- 6-8 h of sleep daily

- Healthy lifestyle measures including diet and physical activity are equally important for non-obese patients with prediabetes.

- People with prediabetes failing to achieve any benefit on lifestyle modifications after 6 months may be initiated on oral antidiabetic agents (OADs):

- Metformin: In younger individuals with one or more additional risk factors for diabetes regardless of BMI, if overweight/obese and having IFG + IGT or IFG $+\mathrm{HbA} 1 \mathrm{c}>5.7 \%$, addition of metformin $(500 \mathrm{mg}$, twice daily) after 6 months of follow-up is recommended.

- Alternatively, alpha-glucosidase inhibitors (AGIs) such as acarbose or voglibose may be initiated if metformin is not tolerated.

- Other pharmacological interventions with pioglitazones, orlistat, vitamin $\mathrm{D}$, or bariatric surgery are not recommended.

- People with prediabetes should be educated on:

- Weight management through optimal diet and physical activity

- Stress management

- Avoidance of alcohol and tobacco

\section{Limited Care}

- The principles for screening are as for recommended care.

- Diagnosis should be based on FPG or capillary plasma glucose if only point-of-care testing is available.

- Using FPG alone for diagnosis has limitations as it is less sensitive than 2-h OGTT in Indians.

Prediabetes

- The principles of detection and management of prediabetes are same as for recommended care.

- Linkages to healthcare system with capacity to provide advice on lifestyle modifications and alignment with on-going support national programs available at community health centres where patients detected with prediabetes can be referred are critical. 


\section{BACKGROUND}

Chronic hyperglycaemia is associated with significantly higher risk of developing diabetes related micro-and macrovascular complications. Early detection of diabetes/prediabetes through screening increases the likelihood of identifying asymptomatic individuals and provides adequate treatment to reduce the burden of diabetes and its complications. Through a computer simulated model on the data from the Anglo-Danish-Dutch study of intensive treatment in people with screen-detected diabetes in primary care (ADDITION-Europe), Herman et al. have demonstrated that the absolute risk reduction (ARR) and relative risk reduction (RRR) for cardiovascular (CV) outcomes are substantially higher at 5 years with early screening and diagnosis of diabetes when compared to 3 years (ARR: 3.3\%; RRR: $29 \%$ ) or 6 years of delay (ARR: $4.9 \%$; RRR: $38 \%)^{\left[{ }^{[12]}\right.}$ Adopting a targeted approach and utilizing lowcost tools with meticulous planning and judicious allocation of resources can make screening cost-effective even in resourceconstrained settings like India. ${ }^{[13]}$ Furthermore, in a systematic review and meta-analysis, screening for type 2 DM (T2DM) and prediabetes has been found to be cost-effective when initiated at around 45-50 years of age with repeated testing every 5 years. ${ }^{[14]}$

Prediabetes is defined as blood glucose concentration higher than normal, but lower than established thresholds for diagnosis of diabetes. People with prediabetes are defined by having IGT (2-h PG in the 75-g OGTT: $140-199 \mathrm{mg} / \mathrm{dL}$ ) or IFG (FPG: $100-125 \mathrm{mg} / \mathrm{dL}$ ). It is a state of intermediate hyperglycaemia with increased risk of developing diabetes and associated CV complications and therefore early detection and treatment of IGT and IFG is necessary to prevent the rising epidemic of diabetes and its associated morbidity and mortality. Although, IDF guideline does not deal with screening and management of prediabetes, the ADA recommends screening for prediabetes and T2DM through informal assessment of risk factors or with an assessment tool. ${ }^{[1]}$ To prevent the progression of pre-diabetes to T2DM, ADA recommends an intensive behavioural lifestyle intervention (BLI) programme (eg, medical nutrition therapy (MNT) and physical activity) in susceptible individuals..$^{[15]}$

Based on the Indian Council of Medical ResearchINdiaDIABetes (ICMR-INDIAB) study conducted in 15 states, the overall prevalence of diabetes and prediabetes was $7.3 \%$ (95\% CI: $7.0,7.5)$ and $10.3 \%(10.0,10.6)$, respectively. ${ }^{[16]}$ Another study conducted among residents of urban areas of east Delhi-The Delhi Urban Diabetes Survey (DUDS) demonstrated a strikingly high prevalence of diabetes (18.3\%: known, $10.8 \%$; newly detected, $7.5 \%)$ and prediabetes (21\% [WHO criteria], 39.5\% [ADA criteria]). ${ }^{[17]}$ Given the high prevalence rates of prediabetes in our country, the RSSDI panel holds the opinion that including screening and management aspects of prediabetes is logical and will provide an important opportunity for prevention of diabetes in India.

\section{Considerations}

The decision about conducting a screening program should be based on the following local factors that were reviewed in Indian context: limited resources, lack of quality assurance in labs, high-risk population for diabetes, large unrecognized burden of undiagnosed diabetes, high prevalence of prediabetes, fast conversion rates from population prediabetes to diabetes, large rural-urban divide, largely sedentary population in urban areas, onset of T2DM at least a decade earlier that in western countries, newer technologies for screening, cost of early detection to the individual, capacity for carrying out screening and capacity to treat/manage screen-positive individuals with diabetes and prediabetes.

\section{Rationale and Evidence \\ Opportunistic screening}

The panel suggests that screening should be opportunistic but not community-based as they are less effective outside health care setting and poorly targeted, i. e., it may fail to identify individuals who are at risk. In a cross-sectional study on 215 participants in a tertiary care hospital in Haryana, opportunistic screening showed that for every seven patients with known diabetes, there are four undiagnosed diabetes patients. ${ }^{[18]}$ In the ICMR-INDIAB study, the ratio of known-to-unknown diabetes was at least 1:1, with rural areas being worse than urban. ${ }^{[16]}$ Opportunistic screening is more cost-effective with better feasibility within the health care system while minimizing the danger of medicalization of a situation. Furthermore, patients diagnosed through this screening have good prognosis over those diagnosed by clinical onset of symptoms. ${ }^{[19]}$

\section{Risk assessment questionnaire}

There are two risks cores specific for Indians developed by Madras Diabetes Research Foundation (MDRF) and by Ramachandran et al. ${ }^{[20]}$ [Annexures 1 and 2]. Both risk scores are validated and are being used widely in our country. The MDRF-Indian Diabetes Risk Score (IDRS) tool has been found to be useful for identifying undiagnosed patients with diabetes in India and could make screening programs more cost-effective. ${ }^{[21]}$ It is also used in several national programs for prevention of not only diabetes but also cardiometabolic diseases such as stroke. Also its applicability in identifying prevalence of diabetes-related complications such as CAD, peripheral vascular disease (PVD), and neuropathy among T2DM patients has been found to be successful. ${ }^{[22]}$ Risk scores by Ramachandran et al. is simple with few risk variables listed and can be applied at any worksite by a paramedical personnel and help identification of the high risk group by the presence of a minimum of 3 or more of the risk variables used in the risk score. ${ }^{[23]}$

\section{Random plasma glucose level}

- The panel endorse the IDF recommendation on the need to measure FPG and perform OGTT based on random plasma glucose levels which are associated 
with the development of diabetes ( $2-\mathrm{h} P G \geq 200 \mathrm{mg}$ / $\mathrm{dL}$ ) or prediabetes ( $2-\mathrm{h} P G \geq 140$ to $<200 \mathrm{mg} / \mathrm{dL}$ ). ${ }^{[24]}$ According to IDF guidelines, FPG values $\leq 100 \mathrm{mg} /$ $\mathrm{dL}$ are considered normal and $\mathrm{FBG}>100 \mathrm{mg} / \mathrm{dL}$ is considered to be at risk of developing diabetes. Further, individuals with FPG between $100-125 \mathrm{mg} / \mathrm{dL}$ have IFG, suggesting an increased risk of developing T2DM. Confirming the FPG levels $\geq 126 \mathrm{mg} / \mathrm{dL}$ by repeating tests on another day, confirm diabetes. ${ }^{[25]}$ In a crosssectional study on 13,792 non-fasting National Health and Nutrition Examination Surveys (NHANES) in participants without diagnosed diabetes, random blood sugar level of $\geq 100 \mathrm{mg} / \mathrm{dL}$ was strongly associated with undiagnosed diabetes. ${ }^{[26]}$ Prediction of diabetes carried out on the basis of this data showed that random blood glucose $\geq 100 \mathrm{mg} / \mathrm{dL}$ was $81.6 \%$ (95\% CI: $74.9 \%$, $88.4 \%$ ) sensitive and 78\% (95\% CI: 76.6\%, 79.5\%) specific to detect undiagnosed diabetes, which is better than current screening guidelines. ${ }^{[27]}$ Evidence from community-based opportunistic screening in India suggests that random capillary blood glucose level of $\geq 110 \mathrm{mg} / \mathrm{dL}$ can be used to identify those individuals who should undergo definitive testing for diabetes or prediabetes. ${ }^{[28]}$ In patients with no history of diabetes or prediabetes, random blood glucose screening is effective in promoting additional screening among high-risk age groups and encourages patients to make lifestyle changes. ${ }^{[29]}$

- The panel suggest that although the present criteria of IFG (100-125 mg/dL) may be sensitive and has lesser variability, measuring 2-h PG levels may give more accuracy and confidence in targeting this population for prevention strategies.

\section{Glycosylated haemoglobin as criteria for screening}

- A meta-analysis of 49 studies involving patients aged $\geq 18$ years reported that $\mathrm{HbAlc}$ as a screening test for prediabetes has lesser sensitivity (49\%) and specificity (79\%) ${ }^{[30]}$ Moreover, the use of ADA recommended HbAlc threshold value of $6.5 \%$ for diagnosis of diabetes may result in significant under diagnosis. ${ }^{[31]}$ The predictive value of $\mathrm{HbA} 1 \mathrm{c}$ for $\mathrm{T} 2 \mathrm{DM}$ depends on various factors such as ethnicity, age, and presence of iron deficiency anaemia (IDA) ${ }^{[32-35]}$ In a cohort study on individuals from Swedish and Middle-East ancestry, $\mathrm{HbA} 1 \geq 48 \mathrm{mmol} / \mathrm{mol}$ had a predictive sensitivity of $31 \%$ and $25 \%$, respectively, for T2DM. ${ }^{[34]}$ Furthermore, HbA1c values $\geq 42$ and $\geq 39 \mathrm{mmol} / \mathrm{mol}$ as predictors for prediabetes were associated with a sensitivity of $15 \%$ and $34 \%$ in individuals of Swedish and $17 \%$ and $36 \%$ in individuals of Middle-East ancestry. Similarly, a systematic review and meta-analysis of 12 studies including 49,238 individuals without $\mathrm{T} 2 \mathrm{DM}$ reveal that $\mathrm{HbA} 1 \mathrm{c}$ values are higher in Blacks (0.26\% (2.8 mmol $/ \mathrm{mol}), \mathrm{p}<0.001)$, Asians (0.24\% $(2.6 \mathrm{mmol} / \mathrm{mol}), \mathrm{p}<0.001)$, and Latinos $(0.08 \%$
$(0.9 \mathrm{mmol} / \mathrm{mol}) ; \mathrm{p}<0.001)$ when compared to Whites. ${ }^{[33]}$ Moreover, significantly high HbA1c levels are observed in patients with IDA when compared to healthy subjects $(5.51 \pm 0.696$ vs $4.85 \pm 0.461 \%, \mathrm{p}<0.001)$ and $\mathrm{HbA} 1 \mathrm{c}$ levels decline significantly after treatment with iron supplements in IDA subjects ( $5.51 \pm 0.696$ before treatment vs $5.044 \pm 0.603$ post-treatment; $\mathrm{p}<0.001){ }^{[35]}$

- The panel recommends the use of HbAlc as sole criteria for screening of diabetes/prediabetes would be inappropriate in most settings in India, at this time. However, HbAlc may be utilized for screening if it is being done from a laboratory known to be well-equipped with external quality assurance. ${ }^{[36]}$

- The panel expresses concerns of high prevalence of anaemia and high prevalence of haemoglobinpathies in certain regions/populations particularly from the North East as these can have significant impact when $\mathrm{HbA} 1 \mathrm{c}$ is used as diagnostic test for screening.

\section{Diagnosis of prediabetes}

- The panel endorses the ADA criteria for diagnosis of prediabetes for Indian context [Table 1]:[1]

\section{Pregnancy as a critical target for diabetes prevention strategies}

- Gestational diabetes mellitus (GDM) is a known risk factor for T2DM. Women with GDM have a 7-fold higher risk of developing T2DM and are at a higher risk of developing metabolic syndrome and CVD. ${ }^{[37,38]}$ Also children of GDM mothers are at higher risk of development of T2DM later in life compared with nonGDM mothers. ${ }^{[39]}$

- Given the high prevalence of GDM in Indian women, early detection and effective treatment can prevent all adverse outcomes of pregnancy and result in a normal and healthy postpartum course for both mother and baby. ${ }^{[40]}$

\section{Children and adolescents: Screening strategies}

- Screening studies in obese adolescents have reported a prevalence of $0.4 \%$ up to $1 \%$ of type 2 diabetes mellitus in obese children $\geq 12$ years. ${ }^{[4]}$

- Overweight (BMI $>90$ percentile) or obese children (BMI $>99.5$ percentile) with familial history of T2DM, children from a predisposed race/ethnicity such as Asian, American Indian, etc., with associated risk factors such as IR, dyslipidemia, polycystic ovarian syndrome must be screened periodically.

- Consistent with the recommendations for screening in adults, children at substantial risk for the development of T2DM should also be tested. The ADA recommends screening in high risk overweight children and adolescents at onset of puberty. The screening must be performed every 2 years from diagnosis using fasting glucose or OGTT.

\section{Rescreening}

- The panel emphasize on striking balance between cost of screening and cost of treating complications. 
- On the basis of expert opinion of the panel, general population should be evaluated for the risk of diabetes by their health care provider on annual basis beginning at age 30 .

- Yearly or more frequent testing should be considered in individuals if the initial screen test results are in the prediabetes range or present with one or more risk factors that may predispose to development of diabetes.

- The panel opine that screening programs should be linked with health care system and ongoing national prevention programs that will facilitate effective and easy identification of people at high-risk of developing diabetes and its complications.

\section{Paramedical personnel}

- Paramedical personnel play a key role as facilitators in imparting basic self-management skills to patients with diabetes and those at risk. They can be actively involved in implementing diet and lifestyle changes, behavioural changes, weight management, pre-pregnancy counselling, and other preventive education.

- Nurses or other trained workers in primary care and hospital outpatient settings can:

- Help in identification of individuals at risk of diabetes

- Help in recognition of symptoms of diabetes, hypoglycaemia, and ketosis

- Help in timely referral of these cases.

- Nurses or nurse educators in secondary and tertiary care settings can:

Perform all the above activities

Help in prevention and treatment of hypoglycaemia

Help in problems with insulin use.

\section{EVIDENCE}

It has been observed that Indians are more prone to diabetes at a younger age and at a lower BMI compared to their Western counterparts. ${ }^{[42,43]}$ The reason for this difference has been attributed to the "Asian Indian phenotype" characterized by low BMI, higher body fat, visceral fat and WC; lower skeletal muscle mass and profoundly higher rates of IR. ${ }^{[44,45]}$ The 10-year follow-up data of the CURES that assessed incident rates of dysglycaemia in Asian Indians are now available. ${ }^{[4]}$ In a cross-sectional study on slum dwellers in Bangalore, prevalence of diabetes and prediabetes was identified as $12.33 \%$ and $11.57 \%$ in people aged $\geq 35$ years. ${ }^{[47]}$ Moreover, in case of female gender, increasing age, overweight and obesity, sedentary lifestyle, tobacco consumption, and diet habits were strongly associated with prevalence of diabetes and prediabetes. Similarly, in a cross-sectional study in Tamil Nadu, prevalence of diabetes and prediabetes was identified as $10.1 \%$ and $8.5 \%$ respectively. ${ }^{[48]}$ Risk factors associated with prediabetes in this study were age of 40 years, male gender, BMI $>23 \mathrm{~kg} / \mathrm{m}^{2}$, WHR for men $>1$ and women $>0.8$, alcohol intake and systolic blood pressure (SBP)
$>140 \mathrm{mmHg}$. Likewise, in a household survey in Punjab, using World Health Organization STEP wise Surveillance (WHO STEPS) questionnaire, prevalence of diabetes and prediabetes were identified as $8.3 \%$ and $6.3 \%$ respectively. ${ }^{[4]}$ Risk factors that were significantly associated with diabetes were age (45-69 years), marital status, hypertension, obesity and family history of diabetes. A study on 163 north Indian subjects proposed severity of IR and family history of diabetes as determinants of diminished beta-cell function leading to diabetes in MS. ${ }^{[50]}$ Predictors of progression to impaired glycaemia were advancing age, family history of diabetes, 2-h PPG, HbA1c, low density lipoprotein (LDL) and HDL, and physical inactivity.

- Despite the escalating burden, the current evidence on the prevention of T2DM and its complications in India is scarce. Though the general practitioners in India are well aware of symptoms and complications of T2DM, they are oblivious regarding the use of standard screening tests resulting in significant delay in diagnosis and treatment. ${ }^{[51]}$

- Considering significant resource constraints together with awareness levels of patients and physicians, there is a need for prevention strategies that are culturally relevant and cost-effective. ${ }^{[52]}$ Following section covers evidence from Indian studies on various strategies that are helpful in detecting and minimizing the risk of development of diabetes and its associated complications.

- Simplified tools for detection of diabetes such as IDRS developed by MDRF and Diabetes Risk Score for Asian Indians devised by Prof. A. Ramachandran are found to be useful for identifying undiagnosed patients with diabetes in India. Use of these tools could make screening programs more costeffective. ${ }^{[20,21]}$ Studies from different regions of India including Jammu, Kashmir, Chennai, Haryana, Delhi, Jabalpur, and Kerala estimated the utility of MDRFIDRS in identifying risk for DM and prediabetes in Indian adult population and found statistically significant association between IDRS and DM patients indicating MDRF-IDRS to be efficient tool to screen and diagnose the huge pool of undiagnosed diabetics in India. ${ }^{[53-58]}$

- It is also found by that identifying the presence of multiple risk factors could be used as a simple measure of identifying people at high risk of diabetes. ${ }^{[59]}$

- The panel suggests that individuals with diabetes or at risk of developing diabetes should be advised on lifestyle changes and implementing strategies focusing on diet, exercise and weight loss to prevent the risk of progression and thus complications of diabetes. ${ }^{[60,61]}$

- Several landmark studies have shown that lifestyle intervention could prevent the progression to T2DM 
by about $30-60 \% .^{[62]}$ Evidence from literature suggests that initial lifestyle interventions are cost-effective ${ }^{[63]}$ and can significantly reduce the incidence of diabetes in Asian Indians with IGT or with combined IGT+IFG. ${ }^{[64-66]}$ The evidence from the randomized, controlled, D-CLIP program showed that adding metformin in a stepwise manner to lifestyle education is an effective method for preventing or delaying diabetes in adults with prediabetes, even in a resource-challenged setting. During the 3 years of follow-up, the RRR was $32 \%$ (95\% CI: 7-50) in intervention participants compared with control. The RRR varied by prediabetes type (IFG+IGT, 36\%; iIGT, 31\%; iIFG, $12 \% ; \mathrm{p}=0.77)$ and was stronger in patients aged $\geq 50$ years, male, or obese. ${ }^{[66]}$

- In patients in whom metformin is contraindicated, AGIs such as acarbose or voglibose may be used, as they confer lesser side effects compared to other OADs. Furthermore, lifestyle intervention with diet and exercise in those with IGT can significantly decrease the incidence of diabetes and its complications ${ }^{[67,68]}$ while providing long-term beneficial effects for up to 20 years. ${ }^{[69]}$

- Several observational studies have reported an association between low levels of vitamin D and increased risk for T2DM, and a few clinical studies that vitamin D could improve the function of beta cells, which produce insulin. However, in the recent D2D study, vitamin D supplementation for a median 2.5 years did not significantly affect prevent T2DM development in a high-risk population. ${ }^{[70]}$

A systematic review and meta-analysis of 50 trials identified that lifestyle intervention reduced risk of progression to diabetes by $36 \%$ over 6 months to 6 years which attenuated to $20 \%$ by the time of follow-up results of the trials were measured. ${ }^{[30]}$ Another systematic review and meta-analysis showed that physical activity in prediabetes subjects improves oral glucose tolerance, FPG and $\mathrm{HbAlc}$ levels, and maximum oxygen uptake and body composition. ${ }^{[71]}$ Results indicated that physical activity promotion and participation slow down the progression of disease and decrease the morbidity and mortality associated with T2DM. Optimal sleep (7-8 h/night) has been shown to maintain metabolic health, aid in weight loss, and increase insulin sensitivity, while short duration $(<5-6 \mathrm{~h})$ or longer duration $(>8-9 \mathrm{~h})$ of sleep was associated with increased risk of diabetes. ${ }^{[72,73]}$ Similar results were observed in a systematic review and meta-analysis of 10 articles which determined that the pooled relative risks for T2DM were 1.09 (95\% CI: 1.04, 1.15) for each 1-h shorter sleep duration among individuals who slept $<7 \mathrm{~h} /$ day and $1.14(1.03,1.26)$ for each 1 -h increment of sleep duration among individuals who slept longer, when compared to 7-h sleep/day. ${ }^{[74]}$

- Interventions predominantly based on counselling and education are found to be effective in preventing/ reducing the risk of developing diabetes and its complication and also helps in improving dietary patterns of individuals with prediabetes and diabetes. ${ }^{[52,75]}$ Mobile phone messaging was found to be an inexpensive and most effective alternative way to deliver educational and motivational advice and support towards lifestyle modification in highrisk individuals. ${ }^{[23]}$ Recent studies have proven the advantages of using mhealth strategies in promotion of prevention of NCDs. The WHO has recommended the use of such strategies for large scale programmes..$^{[76,77]}$

- Dietary interventions such as high-carbohydrate, lowfat diet, ${ }^{[78]}$ fibre-rich, ${ }^{[79]}$ and protein-rich diet $^{[80,81]}$ were found to have definite role in prevention of diabetes. Furthermore, components of whole grains, and fruit and green leafy vegetables such as cereal fibre and magnesium, are consistently associated with lower risk of developing T2DM. ${ }^{[80]}$

- Evidence from the CURES and Prevention Awareness Counselling and Evaluation (PACE) diabetes project suggests that awareness and knowledge regarding diabetes is inadequate among patients in India and implementation of educational programs at massive level can greatly improve the awareness on diabetes and its associated CVD ${ }^{[82,83]}$ Moreover, mass awareness and screening programs through community empowerment were found to effectively prevent and control diabetes and its complications such as foot amputations. ${ }^{[84]}$

- A prospective, randomized controlled study of Fenugreek conducted in nondiabetic people with prediabetes demonstrated a significant reduction in FPG, PPG and LDL and showed insulinotropic effect. ${ }^{[85]}$

- Currently, the role of yoga and fenugreek in the prevention of diabetes is being evaluated in the Indian prevention of Diabetes Study by RSSDI.

\section{IMPLEMENTATION}

A clear and transparent decision should be made about whether or not to endorse a screening strategy. If the decision is in favour of screening, this should be supported by local protocols and guidelines, and public and health-care professional education campaigns. 


\section{Treatment 1: Medical Nutrition Therapy (MNT) and Lifestyle Modifications}

\section{ReCOMmendations}

\section{Recommended Care}

MNT

- The nutrition chart and support should be made in conjunction with a trained nutritionist along with physician/diabetologist.

- Carbohydrates

- Carbohydrate content should be limited to $50 \%-60 \%$ of total calorie intake.

- Complex carbohydrates should be preferred over refined products.

- Low glycaemic index (GI) and low glycaemic load (GL) foods should be preferred.

- Quantity of rice (GI: 73) should be limited as it has high GI; Brown rice (GI: 68) should be preferred over white rice.

- Fibre intake: 25-40 gm per day.

- Proteins

- Protein intake should be maintained at about $15 \%$ of total calorie intake.

- Quantity of protein intake depends on age, sarcopenia and renal dysfunction.

- Non-vegetarian foods are sources of high quality protein, however intake of red meat should be avoided.

- Fats

- Fat intake should be limited ( $<30 \%$ of total calorie intake).

- Oils with high monounsaturated fatty acid (MUFA) and polyunsaturated fatty acid (PUFA) should be used.

- Use of 2 or more vegetable oils is recommended in rotation.

- For non-vegetarians, consumption of 100-200 g of fish/week is advised as good source of PUFA and for vegetarians, vegetable oils (soybean/ safflower/sunflower), walnuts and flaxseeds are recommended.

- Avoid consumption of foods high in saturated fat (butter, coconut oil, margarine, ghee).

- Saturated fatty acids (SFAs) intake should be less than $10 \%$ of total calories/day ( $<7 \%$ for individuals having high triglycerides).

- Use of partially hydrogenated vegetable oils (Vanaspati) as the cooking medium should be avoided.

- Reheating and refrying of cooking oils should be avoided.

- Food groups and patterns

- Diet rich in fruits, leafy vegetates, nuts, fibre, whole grains and unsaturated fat is preferred.

- Food plate should include pulses, legumes, unprocessed vegetables and low fat dairy.

- Extreme diets including low-carbohydrate ketogenic must be planned and executed following consultation with physician and nutritionist, and for a short period.

- Overall salt consumption should be $<5 \mathrm{~g} /$ day (with sodium consumption $<2300 \mathrm{mg} /$ day).

- Avoid or decrease alcohol intake.

- Smoking cessation should be advised to all. Smoking cessation therapies may be provided under observation for patients who wish to quit in step-wise manner

- Sugar sweetened beverages are best avoided.

- Artificial sweeteners may be consumed in recommended amounts.

- Meal plans with strategic meal replacements (partial or full meal replacements) may be an option under supervision when feasible.

- Lifestyle modifications

- Recommended care could be imparted by physician and diabetes educator.

- Careful instructions should be given for initiating exercise programme. Help of a physical instructor can be taken.

- Lifestyle advice should be given to all people with T2DM at diagnosis. It should be an effective option for control of diabetes and increasing CV fitness at all ages and stages of diabetes.

- Lifestyle intervention is a cost-effective approach in prevention of T2DM.

- Lifestyle interventions should be reviewed yearly or at the time of any treatment or at every visit.

- Advise people with T2DM that lifestyle modification, by changing patterns of eating and physical activity, can be effective in managing several adverse risk factors related to T2DM.

- Physical activity should be introduced gradually, based on the patient's willingness and ability and the intensity of the activity should be individualized to the specific goals

- A minimum of $150 \mathrm{~min} /$ week of physical activity is recommended for healthy Indians in view of the high predisposition to develop T2DM and CAD

- $\geq 30$ min of moderate-intensity aerobic activity each day

- 15-30 min of work-related activity

- 15 min of muscle-strengthening exercises (at least 3 times/week)

- While effect of yogic practices is encouraging, it should not replace aerobic exercise.

- Use of monitoring tools like accelerometers, GPS units, pedometers, mobile based apps or devices to measure the intensity and duration of physical activity may be encouraged.

Behavioural lifestyle intervention (BLI)

- BLI involves patient counselling for strategies such as tailoring goals, self-monitoring and stimulus control.

- BLI approaches have shown to improve adherence to lifestyle changes and achieve more sustained effects.

- Diabetes self-management support is important and could be done with physician or educator with small groups or face-to-face discussions in chat rooms. 


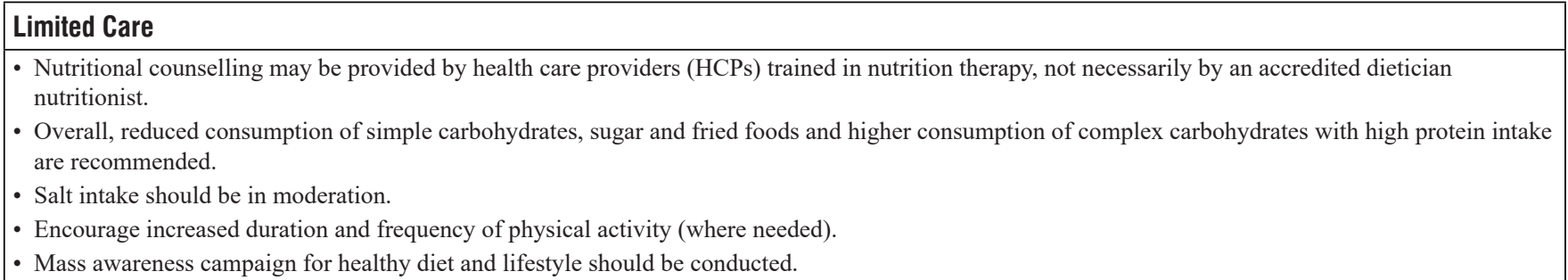

\section{BACKGROUND}

Unhealthy diet and sedentary lifestyle have been identified as fundamental modifiable risk factors in T2DM. Rapid urbanization and rampant availability of westernized, processed foods that contain high amounts of refined carbohydrates, saturated fats and added sugars have dramatically changed the local food environment in India.

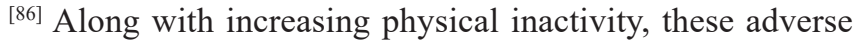
dietary changes have been associated with detrimental influences on the onset and progression of T2DM in India. ${ }^{[87-89]} \mathrm{MNT}$ is a systematic approach to optimize dietary intake in order to achieve metabolic control and maximize favourable treatment outcomes in T2DM. Conceptually, MNT involves counselling and recommendations from a registered dietician (RD) under the regular supervision of the consulting diabetologists.

Current global clinical practice guidelines for T2DM from the ADA, American Association of Clinical Endocrinologists (AACE) and IDF advocate the importance of integrating MNT in the management of T2DM as a first-line therapy and provide consistent recommendations for day-to-day nutritional requirements. ${ }^{[00,91]} \mathrm{MNT}$ is a lifestyle transforming process that is beyond calorie restriction and portion control. Implementation of MNT in India is challenging owing to its cultural and culinary diversity. Consumption of high amounts of carbohydrates including ghee-laden sweets loaded with sugar or jaggery are inherent to the standard Indian diet and have religious significance, thus escalating the challenges of restricting carbohydrate intake. Therefore designing individualized diet plans as a part of MNT in India should consider regional, cultural, economic and agricultural factors as these have a marked influence on the acceptance of MNT by the patient.

\section{Role of medical nutrition therapy in prevention and management}

Dietary counselling and adherence of healthful, calorierestricted diet and regular exercise have been associated with lower rates of incident diabetes in Indian men with impaired glucose tolerance. Community health programs and implementation of MNT-based model meals in rural and urban populations from South and North of India have shown favourable changes in dietary patterns and improvements in several parameter including BMI, waist circumference, fasting blood glucose and so on. ${ }^{[75,92,93]}$ A stepwise Diabetes Prevention Program lowered the 3-year risk of diabetes by $32 \%(95 \%$ CI: 7,50$)$ in obese Asian Indian adults with any form of prediabetes. ${ }^{[66]}$ These studies including few others involving Indians with risk factors for diabetes reported benefits of dietary approaches such as high consumption fibre-rich foods, high-protein meal replacements, or replacement of polished white rice with whole grain brown rice and increased intake of fruits and vegetables. ${ }^{[94,95]}$

The landmark lifestyle intervention program, Look Ahead examined the effects of calorie-restricted diet and reduce intake of high-GI carbohydrates such as sugar, flavoured beverages and high calorie snacks on glycaemic control and prevention of $\mathrm{CV}$ complications. At 11 years, participants benefited from the regimented diet and had an average weight loss of 5\% along with substantial improvements in $\mathrm{HbA} 1 \mathrm{c}$ levels, blood pressure, lipid profile and overall fitness and well-being. ${ }^{\left[{ }^{[6]}\right]}$ In a year-long prospective randomized study from India, individuals with T2DM, randomized to MNT, achieved significant lowering of $\mathrm{HbAlc}$ and all lipid parameters, especially triglyceride levels. This study involved 20 dieticians and reported the success of a guided, evidence-based, individualized MNT versus usual diabetes care. ${ }^{[97]}$ Based on these clinically relevant observations in the Indian population, the RSSDI recommends the adoption of dietician-guided MNT as an integral component of diabetes management [Figure 1]. The MNT and lifestyle modifications should be individualized based on diseases profile, age, sociocultural factors, economic status, and presence of sarcopenia and organ dysfunction.

\section{Rationale and Evidence}

\section{Carbohydrate monitoring}

Meal planning approaches should include carbohydrate counting, exchanges or experience based estimation and measurement of GI and GL to monitor the amount of carbohydrate in food and understand its physiological effects of high-carbohydrate diets. ${ }^{[98,99]}$

\section{High-carbohydrate, low-fat diets}

Although there is a dichotomy in recommendations with regard to high-or low-carbohydrate diets, historic data from India suggest the metabolic benefits of high- 


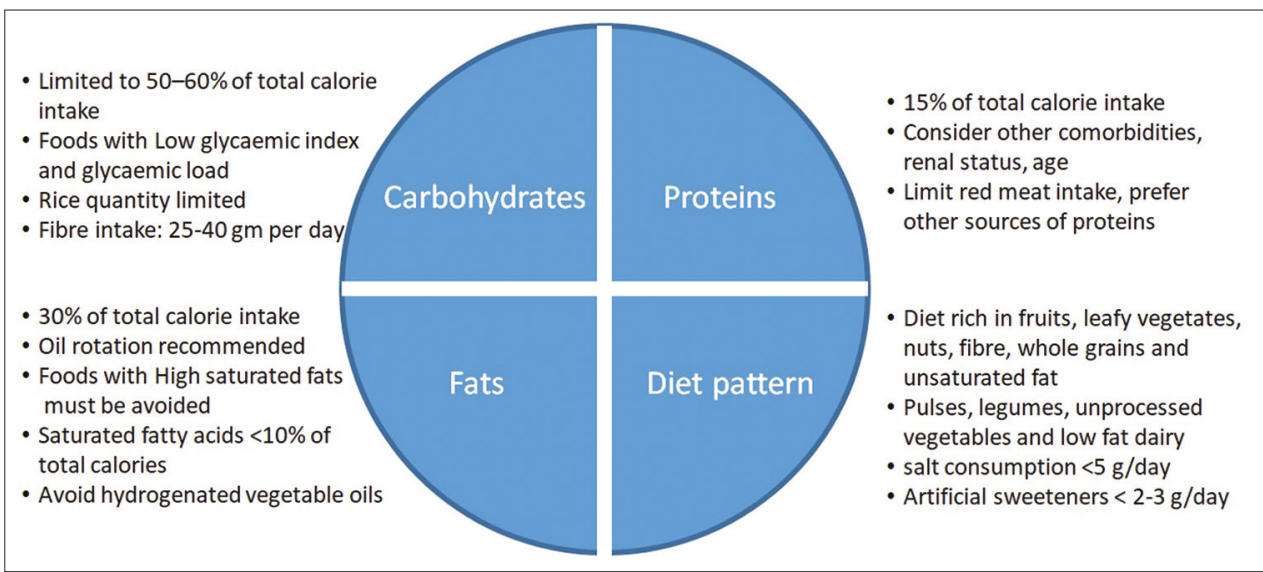

Figure 1: Recommendation for MNT in patients with T2DM. MNT: Medical nutrition therapy; T2DM: Type 2 diabetes mellitus

carbohydrate, high-fibre, low-fat diets as opposed to a highfat, low-carbohydrate diet. ${ }^{[100,101]}$ Recent studies support the implementation of a long-term high-carbohydrate high-fibre diet in promoting weight loss, improving glycaemic control, and lowering CV risk. ${ }^{[102-105]}$ High carbohydrate diets should comprise large amounts of unrefined carbohydrate and fibre such as legumes, whole grains, unprocessed vegetables, and fruits. ${ }^{[87,106,107]}$ High carbohydrate diet regimens in T2DM patients have been associated with favourable weight loss and reductions in plasma glucose, HbA1c and LDL levels with good adherence and sustainability, comparable with low carbohydrate diets. The concern of possible untoward effect of high carbohydrate diet on lipid profile (increase in triglycerides and reductions in HDL) and $\mathrm{CV}$ risk can be mitigated by lowering the glycaemic index of diets by incorporating fibre-rich foods. ${ }^{\text {[99] }}$

- Cross-sectional data from the CURES suggests that Indians consume high amounts of refined grains ( $47 \%$ of total calories), which is associated with significant increases in waist circumference $(\mathrm{p}<0.0001)$, systolic blood pressure $(p<0.0001)$, diastolic blood pressure $(p=0.03)$, fasting blood glucose $(p=0.007)$, serum triglyceride $(p<0.0001)$, lower HDL $(p<0.0001)$, and insulin resistance $(p<0.001)$. Further, Indians who consumed refined grains were more predisposed develop metabolic syndrome (odds ratio [OR]: 7.83; 95\% confidence interval [CI]: 4.72, 12.99) and insulin resistance versus those consumed lower quantities. ${ }^{[108]}$

- In an assessment of the quality and type of carbohydrates in a subset of patients from the CURES study, consumption of refined grain (OR: 5.31 ; 95\% CI: 2.98 , 9.45; $\mathrm{p}<0.001$ ), total carbohydrate (OR: 4.98; 95\% CI: 2.69, 9.19; $\mathrm{p}<0.001$ ), GL (OR: 4.25; 95\% CI: 2.33, 7.77; $<<0.001$ ), and GI (OR: $2.51 ; 95 \%$ CI: 1.42, 4.43; $\mathrm{p}=0.006$ ) positively correlated with the risk of T2DM. In contrast, high intake of dietary fibre showed an inversely correlation with T2DM (OR: 0.31; 95 \% CI: 0.15, 0.62; $\mathrm{p}<0 \cdot 001){ }^{[107]}$
- Additional analysis of the data from the CURES study population revealed the detrimental dietary habits among South Indian adults (daily energy intake: carbohydrates [64\%], fat [24\%], protein [12\%]) that escalates the risk of T2DM. It was observed that refined cereals contributed to nearly $46 \%$ of total energy intake, followed by visible fats and oils (12.4\%), pulses and legumes (7.8\%), and intake of micronutrient-rich foods (fruits, vegetables, fish etc.) was inadequate and below the recommended standards of FAO/WHO. ${ }^{[109]}$

- Given that carbohydrates are an inherent part of the staple Indian diet and Indians habitually tend to consume high amounts of carbohydrates, improving the quality of carbohydrates in the diet by replacing high-GI carbohydrates with fibre-rich, low GI counterparts. ${ }^{[110]}$ It was observed that consumption of brown rice significantly reduced 24-h glycaemic response 24-h $(p=0.02)$ and fasting insulin response $(\mathrm{p}=0.0001)$ in overweight Asian Indians. ${ }^{[111]}$

- $\quad$ Replacement of white rice with brown rice was found to be feasible and culturally appropriate in Indian overweight adults and correlated with lower risk of T2DM. ${ }^{[112]}$ Fortification of humble Indian dishes with fibre-rich alternatives, for example adding soluble fibre in the form of oats in upma or improving the glycaemic quality of Indian flatbreads (rotis or chapattis) by adding wheat flour with soluble viscous fibres and legume flour have shown favourable outcomes on the lipid profile and postprandial glucose and insulin responses in T2DM patients. ${ }^{[113-116]}$

- Sugar and sugar-sweetened beverages increase the dietary GL. Overall, the consumption of total sugar (25.0 kg/capita) among Indians exceeds the average global annual per capita consumption $(23.7 \mathrm{~kg})$.

- Consumption of sweets, sweetened beverages (e. g. lassi, aamras) or addition of sugars in curries, gravies etc. have customary and regional importance in India. ${ }^{[17]}$ It is observed that in urban South India, the added sugars in 
hot beverages (tea or coffee) majorly contribute to sugar intake and account for around $3.6 \%$ of total GL. ${ }^{[107]}$

\section{Low-carbohydrate, ketogenic diet}

Low-carbohydrate diets may be particularly beneficial in patients with impaired glucose tolerance, and obesity, however to balance the macronutrient content, these diets tend to be high in fats and proteins. Therefore, while adopting such diets, fat intake should occur mainly in the form of MUFA with a parallel decrease in saturated fatty acids (SFAs) and trans fatty acids (TFAs). As the metabolic pathways of carbohydrates and fats are interlinked, low carbohydrate diets that are high on fats and protein are associated with long-term effects such as ketosis, and adverse lipid, and renal effects. ${ }^{[118]}$

- $\quad$ Evidence suggest that T2DM patients on low carbohydrate diet achieve favourable outcomes due to reduced energy intake and prolonged calorie restriction and not due to low carbohydrate intake. Obese T2DM patients should therefore consider switching to a low-carbohydrate diet designed based on calorie restriction as well as regulated intake of fats to reduce the incidence of T2DM and myocardial infarction. ${ }^{[119,120]}$ These diets should be considered for a limited period only.

- In a small study, overweight patients with T2DM were randomized to a very low carbohydrate ketogenic diet and lifestyle modifications such as physical activity, sleep etc. had significantly improved their glycaemic control $(p=0.002)$ and lost more weight $(p<0.001)$ than individuals on a conventional, low-fat diabetes diet program. ${ }^{[121]}$

- In another similarly designed randomized controlled trial, overweight individuals with T2DM or elevated HbA1c levels on very low carbohydrate ketogenic diet for a period of 12 months had significant reductions in HbA1c levels $(p=0.007)$ and body weight $(p<0.01)$ than participants on moderate-carbohydrate, calorie-restricted, low-fat diet. ${ }^{[122]}$

- In a 24-week interventional study, a low-carbohydrate ketogenic diet in patients with T2DM favourably improved body weight, glycaemic and lipid profiles in patients with T2DM as compared with patients on a low calorie diet. ${ }^{[123]}$

\section{Low glycaemic index of pulses and pulse-incorporated cereal foods}

As compared with other Western or Asian diets, traditional Indian diets comprising dal, roti, rice, and curry provide a wholesome supply of balanced, mixed nutrients. The mix of various pulses and legumes in a standard Indian meal offer variations in the glycaemic and insulinaemic indices that are attributed to the nature of available and non-available (non-starchy polysaccharides) carbohydrates in the foods and alterations in rates of carbohydrate absorption. ${ }^{[124,125]}$ Inclusion of grams and pulses in rice or wheat-based starchy high GI diets reduces the glycaemic index and brings satiety along with adequate supply of calories. Meals with mixed sources of cereals, pulses and legumes contribute to regulation of insulin and glycaemic responses.

- Combining acarbose in regular daily diets was associated with significant decline in postprandial blood glucose in T2DM patients, including those who failed prior treatment with OADs. $^{[126]}$

- Similarly, consumption of adai dosa (a type of Indian pancake with $75 \%$ pulses and $25 \%$ cereals) versus normal diet ( $75 \%$ cereal and $25 \%$ pulses) was associated with reduction in body weight and significant $(\mathrm{p}<0.01)$ lowering of $\mathrm{HbA} 1 \mathrm{c} .{ }^{[127]}$

- Inclusion of nuts (almond, walnuts, cashews, pistachios, hazelnuts) in diet corresponding to approximately $56 \mathrm{~g}$ ( $1 / 2$ cup) of nuts was associated with significant reduction in HbA1c (mean difference: - 0.07\% [95\% CI: -0.10 , $-0.03 \%$ ]; $\mathrm{p}=0.0003$ ) and fasting glucose (mean difference: $-0.15 \mathrm{mmol} / \mathrm{L}$ [95\% CI: $-0.27,-0.02 \mathrm{mmol} / \mathrm{L}] ; \mathrm{p}=0.03$ ) in individuals with T2DM versus isocaloric diets without nuts. The improvement was mainly attributed to lowering of GI due to replacement by nuts. ${ }^{[128]}$

- In an analysis of dietary patterns in India, diets rich in rice and pulses were associated with lower risk of diabetes versus diet models that had more sweets and snacks. ${ }^{[129]}$

- Legumes such as chickpeas are also low glycaemic foods and when substituted for similar serving of egg, baked potato, bread, or rice lower the risk of T2DM and may be beneficial in elderly individuals with $\mathrm{CV}$ risk. ${ }^{[130]}$

\section{Consumption of oils among Indian population}

- In the rural South Indian population from the CURES study, highest intake of fats directly correlated with risk of abdominal obesity $(p<0.001)$, hypertension $(p=0.04)$, and impaired fasting glucose $(\mathrm{p}=0.01)$. In particular, sunflower oil was found to be most detrimental when compared to traditional oils and palmolein. ${ }^{[131]}$

- $\quad$ Supporting this finding, the risk of metabolic syndrome was higher among users of sunflower oil (30.7\%) versus palmolein $(23.2 \%)$ or traditional (groundnut or sesame) oil $(17.1 \%, p<0.001)$ in Asian Indians. Higher percentage of linoleic acid PUFA in sunflower oil was correlated with the risk of metabolic syndrome. ${ }^{[132]}$ The observations from these studies are preliminary and should be further investigated.

- Managing dietary intervention by replacing refined cooking oils with those containing high percentage of MUFA (canola and olive oil) in Asian Indians with nonalcoholic fatty liver disease was associated with significant reduction in body weight and BMI ( $\mathrm{p}<0.01$, olive oil), improvements in fasting insulin level and homeostasis model of assessment for insulin resistance and $\beta$-cell function $(p<0.001$, olive oil), increase in high-density lipoprotein level $(p=0.004$, olive oil), and decrease in fasting blood glucose $(p=0.03)$ and triglyceride $(p=0.02)$ level (in canola group). ${ }^{[133]}$ 
- Increasing use of saturated fat, low intake of n-3 PUFAs, and increase in TFAs was observed among India patients with T2DM and obesity and it is recommended that improving quality of fats in diet (more MUFAs and omega 3 PUFAs) would be beneficial in T2DM. ${ }^{[134,135]}$

- Repeated heating/frying or reusing of oils at high temperature, which is a common practice in India should be avoided as it, induces chemical changes that increases the amounts of harmful TFAs, which greatly elevate the risk of CV complications in T2DM patients. ${ }^{[136,137]}$

\section{Fibre and diabetes mellitus}

- Increasing the intake of dietary fibres is known to have a favourable effect on the overall metabolic health. Fibrerich foods contain complex carbohydrates that are resistant to digestion and thereby reduce glucose absorption and insulin secretion. ${ }^{[79,138,139]}$

- In overweight or obese patients with T2DM, a low glycaemic-index, high-fibre diet significantly $(\mathrm{p}<0.001)$ reduces glucose and insulin area under the curve compared with high-glycaemic index, high carbohydrate diets. The favourable effects on postprandial glucose and insulinemia were sustained through an entire day. ${ }^{[140]}$

- Consumption of high-carbohydrate, low-GI diets that contain high proportions of dietary fibres also mitigate the risk of increase in serum triglyceride levels which is a common consequence of high-carbohydrate diet. ${ }^{[99]}$

- Intake of both soluble and insoluble fibres have been associated with increased post-meal satiety and decrease in consequent hunger episodes. ${ }^{[141]}$

- In randomized, cross-over study in 56 healthy Indian participants, consumption of flatbreads with addition of fibrous flour such as chickpea (15\%) and guar gum (3\% or $4 \%$ ) to wheat flour significantly reduced postprandial glucose $(\mathrm{p}<0.01)$ and postprandial insulin $(\mathrm{p}<0.0001)$ when compared with flatbreads made from control flour (100\% wheat flour). ${ }^{[113]}$

- In a dietary assessment study in urban Asian Indians with T2DM, low consumption of dietary fibres ( $<29 \mathrm{~g} /$ day) was associated with higher prevalence of hypercholesterolemia $(p=0.01)$ and higher LDL $(p=0.001)$ than individuals with greater median intake of fibres. ${ }^{[142]}$

- In a randomized study, daily consumption of $3 \mathrm{~g}$ of soluble fibre from $70 \mathrm{~g}$ of oats in form of porridge or upma for 28 days in mildly hypercholesterolaemic Asian Indians was associated with significant reduction in serum cholesterol $(\mathrm{p}<0.02)$ and LDL $(\mathrm{p}<0.04)$ versus control group (routine diet). ${ }^{[14]}$

- From a meta-analysis of 17 prospective cohort studies, an inverse relation was observed between dietary intake and risk of T2DM based on which it was recommended that intake of $25 \mathrm{~g} /$ day total dietary fibre may T2DM. ${ }^{[143]}$

\section{Physical activity}

- The International Physical Activity Questionnaire-Long Form and accelerometer can be used to measure and monitor the intensity of physical activity. ${ }^{[144]}$

- Physical inactivity is regarded as a major risk factor for T2DM and evidence suggests that adequate physical activity may reduce the risk by up to $27 \% .^{[110,145]}$

- Structured exercises have found to significantly reduce $(p<0.001)$ post interventional HbA1c levels versus control group and this effect was independent of body weight. ${ }^{[146]}$

- In the Indian Diabetes Prevention Program report, lifestyle modification that included a minimum of 30 min/day of physical labour, exercise, or brisk walking showed significant relative risk reductions for T2DM either alone $(28.5 \% ; \mathrm{p}=0.018)$ or in combination with metformin $(28.2 \% ; \mathrm{p}=0.022)$ versus the control group. ${ }^{[65]}$

- In a cross-sectional comparative study, South Asians were found to need an additional $10-15 \mathrm{~min} /$ day of moderateintensity physical activity more than the prescribed 150 $\mathrm{min} /$ week to achieve the same cardio-metabolic benefits as the European adults. ${ }^{[147]}$

- Resistance training either alone or in combination with aerobic exercises or walking has also shown to significantly improve risk factors of T2DM such as waist circumference, abdominal adiposity, HDL levels etc. $^{[148-150]}$

- $\quad$ Based on all available evidence, the ADA and IDF recommend a total of at least 150 min of moderateintensity physical activity per week that can be a combination of aerobic activities (such as walking or jogging) or resistance training. ${ }^{[151]}$

- For Asian Indians who are predisposed to develop T2DM or CV risks, an additional $60 \mathrm{~min}$ of physical activity each day is recommended, although there is limited data to support this recommendation. ${ }^{[152]}$

\section{Behavioural lifestyle intervention}

- $\quad$ BLI involves patient counselling for strategies such as tailoring goals, self-monitoring, stimulus control etc. that would help motivate patients to integrate the lifestyle management measures into their day-to-day life and identify and manage potential lapses. ${ }^{[153]}$

- BLI approaches have shown to improve adherence to lifestyle changes and achieve more sustained effects. ${ }^{[154]}$

- In patients with T2DM implementation of a six-month BLI program was reported to significantly reduce $\mathrm{HbA} 1 \mathrm{c}$ levels from baseline at 3 months $(-1.56 \pm 1.81, \mathrm{p}<0.05)$ and 6 months $(-1.17 \pm 2.11, \mathrm{p}<0.05)$. The BLI used cognitive behaviour therapy that mainly involved monitoring of carbohydrate intake (using diet charts) and setting targets for weight loss and physical activity across of 8 sessions (4 face-to-face and 4 telephone sessions) administered by clinical dietitians. ${ }^{[155]}$

- $\quad$ BLI using smartphone or paper-based self-monitoring of patient behaviours on weight loss and glycaemic control (based on Look AHEAD study) in overweight or obese 
adults with T2DM showed significant improvements in $\mathrm{HbAlc}(\mathrm{p}=0.01)$ at 6 months and meaningful weight loss that was not significant. ${ }^{[156]}$

- A systematic review of randomized studies evaluating lifestyle-based interventions for T2DM found that robust behavioural strategies were essential for successful implementation of such prevention programs. This study reviewed the Indian Diabetes Prevention Programme that included individual patient counselling and goal setting for diet and exercise. ${ }^{[65,157]}$

\section{Treatment 2: Oral Antidiabetic Agents}

\section{ReCOMMENDATIONS}

\section{Recommended Care}

General Principles

- Metformin should be initiated in combination with lifestyle interventions at the time of diagnosis.

- Maintain support for lifestyle measures throughout

- Consider each initiation or dose increase of OADs as a trial, monitoring the response through glucose monitoring (FPG, PPG, self-monitoring of blood glucose [SMBG] or HbA1c) every 2-3 months.

- Consider CV/heart failure risk, renal/hepatic (NASH) risk while deciding therapy

- Patient-centric approach: consider cost and benefit risk ratio when choosing OADs

- Customise therapy focusing on individualised target HbA1c for each patient based on: age, duration of diabetes, comorbidities, cost of therapy, hypoglycaemia risk, weight gain, durability

- Consider initiating combination therapy if the HbA1c $>1.5$ above the targetInitial Therapy

- Metformin should be initiated in combination with lifestyle interventions at the time of diagnosis unless contra-indicated or not tolerated.

- If eGFRis between $45-30 \mathrm{~mL} / \mathrm{min} / 1.73 \mathrm{~m}^{2}$ : reduce dose of metformin by $50 \%$; stop metformin if eGFR $<30 \mathrm{~mL} / \mathrm{min} / 1.73 \mathrm{~m}^{2}$. Closely monitor renal function every 3 months

- Other options: sulfonylurea (or glinides), dipeptidyl peptidase-4 (DPP-4) inhibitors, SGLT2 inhibitors, or AGIs can be used initially for cases where metformin is contraindicated or not tolerated

- In some cases, dual therapy may be indicated initially if it is considered unlikely that single agent therapy will achieve glucose targets

- Dualtherapy: Patient-centric approach

- If glucose control targets are not achieved: Add sulfonylurea or thiazolidinediones (TZDs) or sodium-glucose cotransporter 2 inhibitors (SGLT2) inhibitor, or DPP-4 inhibitor, or AGI

- Individualise patient care based on comorbidities

- Tripletherapy: Patient-centric approach

- If glucose targets are not achieved with two agents: start third oral agent-AGI, DPP-4 inhibitor, SGLT2 inhibitor, or TZDs (depending on second-line agent used) or start insulin or glucagon-like peptide 1 (GLP-1) agonists

- Intensification of therapy: Patients not achieving glycemic targets on 3 oral agents

- Consider GLP-1 agonists or insulin if glucose targets are not achieved with OADs

- Exceptionally, addition of fourth agent may be considered.

- In the presence of IR, addition of TZDs may be considered

- For patients with established atherosclerotic cardiovascular disease (ASCVD), heart failure, diabetic kidney disease (DKD) or in need of weight reduction consider using SGLT2 inhibitors

- If postprandial hypoglycaemia is the issue AGI, glinides or SGLT2 inhibitors may be considered

- In elderly patients with increased risk of hypoglycaemia, use a DPP-4 inhibitor as an alternative to sulfonylurea

\section{Limited Care}

- The principles are same as for recommended care along with considerations for cost and availability of generic therapies. In resource constrained situations, sulfonylurea or metformin or TZDs may be used.

- Newer sulfonylureas have benefit of low cost and reduced hypoglycaemia (than older OADs); comparable CV safety with DPP4i may be considered. TZDs have established CV safety and may be considered as add on to metformin. 


\section{BACKGROUND}

T2DM occurs due to a complex interaction between genetic inheritance and risk factors such as obesity and sedentary lifestyle. ${ }^{[158]}$ Diminished insulin secretion, incretin deficiency/resistance, upregulated lipolysis, increased glucose reabsorption from kidney, along with downregulated glucose uptake, neurotransmitter dysfunction, increased hepatic glucose production, and glucagon secretion are the reported metabolic derailments that contribute to hyperglycaemia and T2DM [Figure 2]. ${ }^{[159]}$ Among Indians, high familial aggregation, central obesity, insulin resistance, and life style changes due to rapid urbanization are the primary causes of T2DM. ${ }^{[160]}$ Greater degree of insulin resistance paired with higher central adiposity compared to Caucasians is a characteristic feature of T2DM in Asian Indians. ${ }^{[161,162]}$

Treatment options for T2DM have been developed in parallel to the increased understanding of underlying pathophysiological defects in T2DM. A patient-centric and evidence-based approach that may take into account all the metabolic derailments accompanying T2DM, is now gaining impetus. Therefore, treatments that target factors beyond glycaemic control, such cardiovascular risks, weight management, along with improvements in quality of life have been introduced. ${ }^{[163]}$ Several guidelines/recommendations provide treatment algorithms on ways in which glucoselowering agents can be used either alone or in combination.
Ideally, treatment decisions should be directed based on glycaemic efficacy and safety profiles, along with impact on weight and hypoglycaemia risk, comorbidities, route of administration, patient preference, as well as treatment costs. ${ }^{[164]}$ Here the guideline is based on clinical evidences and provides overview on available OADs. The treatment algorithms in this chapter attempt to provide practical recommendations for optimal management of T2DM in Asian Indians.

\section{Considerations}

The decision on choice of OAD therapy in T2DM patients is based on the cost, safety, and efficacy and comorbidities that were reviewed in Asian Indian context.

\section{Rationale and Evidence \\ Oral antidiabetic agents [Table 2] \\ Biguanides}

Metformin remains the first choice in the management of patients with T2DM. ${ }^{[165]}$ Metformin is efficacious in managing hyperglycaemia, increasing insulin sensitivity, along with beneficial effects in reducing cardiovascular and hypoglycaemia risk, improving macrovascular outcomes, and lowering mortality rates in T2DM. ${ }^{[166]}$ Metformin is a complex drug that exerts its action via multiple sites and several molecular mechanisms. Metformin is known to down regulate the hepatic glucose production, act on the gut to

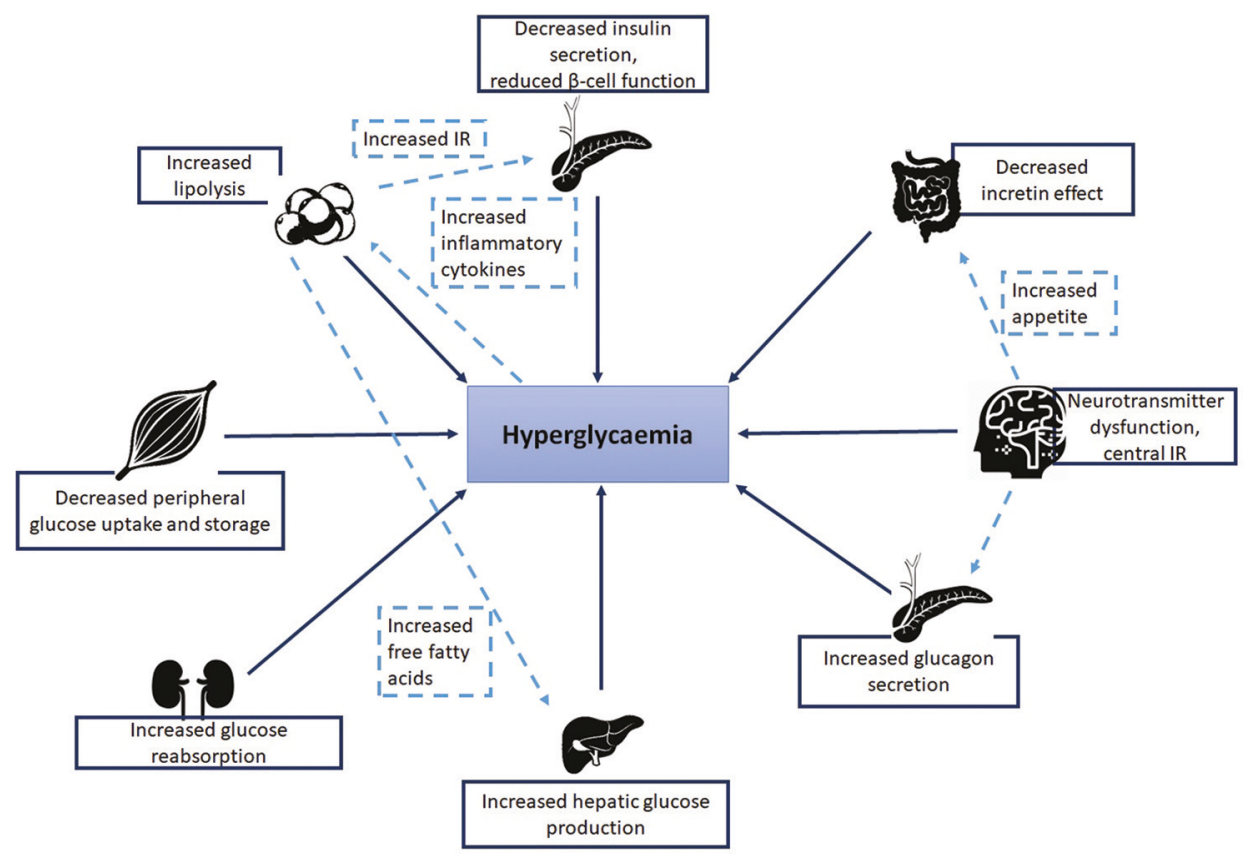

Figure 2: Metabolic derailments in T2DM. ${ }^{[159]}$

IR: Insulin resistance; T2DM: Type 2 diabetes mellitus 
increase glucose utilisation, enhance insulin, increase GLP-1 and alter the microbiome. ${ }^{[167]}$ TheUK Prospective Diabetes Study (UKPDS) Group study in over weight T2DM patients suggested that intensive glucose control that metformin lowered the risk of diabetes-related endpoints, diabetesrelated deaths, and all-cause mortality in overweight T2DM patients, compared to insulin and sulphonylureas. ${ }^{[168]} \mathrm{A}$ 10-year follow-up study of the UKPDS reported continued benefit following intensive glucose control with metformin in terms of reduced diabetes-related endpoints, diabetesrelated deaths, and all-cause mortality. ${ }^{[169]}$ Along with substantial improvements in hyperglycaemia, metformin improved endothelial dysfunction, oxidative stress, insulin resistance, lipid profiles, and fat redistribution. ${ }^{[170]}$ Owing to the concerns of lactic acidosis and gastrointestinal effects (nausea, vomiting diarrhoea and flatulence) metformin should be used cautiously in patients with renal insufficiency or elderly patients. In patients with an eGFR $<60 \mathrm{~mL} /$ $\min / 1.73 \mathrm{~m}^{2}$ metformin can be used, but should not be initiated in patients with an eGFR of 30 to $45 \mathrm{~mL} / \mathrm{min} / 1.73$

\begin{tabular}{lc}
\hline $\begin{array}{l}\text { Table 1: Glycaemic values for Indian patients with type } \mathbf{2} \\
\text { diabetes mellitus }\end{array}$ \\
\hline Glycaemic parameter & Values \\
\hline FPG (mg/dL) & $100-125$ \\
2-h PPG during 75-g OGTT (mg/dL) & $140-199$ \\
HbA1c (\%) & $5.7-6.4$ \\
\hline
\end{tabular}

2-h PPG: 2-h postprandial glucose, FPG: Fasting plasma glucose, HbA1c: Glycosylated haemoglobin, OGTT: Oral glucose tolerance test $\mathrm{m}^{2}$ and must be contraindicated in patients with an eGFR $<30 \mathrm{~mL} / \mathrm{min} / 1.73 \mathrm{~m}^{2}{ }^{[164]}$

\section{Sulfonylureas}

Sulfonylureas are second line agents used in patients with T2DM patients who are not obese. Sulfonylureas are insulin secretagogues that act on the ATP-sensitive $\mathrm{K}+$ channels on the $\beta$ cells and stimulate endogenous insulin secretion. ${ }^{[171]}$ As a single therapy, sulfonylureas are efficacious in lowering fasting plasma glucose and HbAlc. However, concerns of modest weight gain and moderate to severe hypoglycaemia and cardiovascular risk limit their clinical benefits. ${ }^{[172]}$ As a consequence of closure of cardiac $\mathrm{K}_{\text {ATP }}$ channel, the use of sulfonylureas have also been related to adverse $\mathrm{CV}$ effects due to impaired hypoxic coronary vasodilation during increased oxygen demands such as acute myocardial ischemia. ${ }^{[173]}$ The use of glibenclamide was associated with an increased risk of in-hospital mortality in patients with diabetes and acute myocardial infarction. ${ }^{[174]}$ Adverse cardiovascular outcomes with sulfonylureas in some observational studies have raised concerns, although findings from recent meta-analysis that included several RCTs reported that sulfonylureas when added to metformin were not associated with allcause mortality and CV mortality. ${ }^{[175]}$ New generation sulfonylureas have demonstrated superior safety, mainly due to reducing hypoglycaemia, and improved cardiac profile. Sulfonylureas particularly gliclazide modified release (MR) and glimepiride have a lower risk of hypoglycaemia and are preferred in south Asian T2DM patients. ${ }^{[176]}$ Caution must be exercised while prescribing sulfonylureas for patients at a

\begin{tabular}{|c|c|c|c|c|}
\hline & Effect on weight & CV effects & $\begin{array}{l}\text { Significant side } \\
\text { effects }\end{array}$ & Summary \\
\hline $\begin{array}{l}\text { Biguanides } \\
\text { Metformin }\end{array}$ & $\begin{array}{l}\text { Weight loss/ } \\
\text { neutral }\end{array}$ & $\begin{array}{l}\text { Beneficial CV effects, reduces } \\
\text { LDL-C and increases HDL-C; } \\
\text { however, reports of caution indicated } \\
\text { particularly in older patients with CHF }\end{array}$ & Lactic acidosis & $\begin{array}{l}\text { First-line drug therapy of } \\
\text { choice for T2DM } \\
\text { Acceptable CV safety profile }\end{array}$ \\
\hline $\begin{array}{l}\text { SU } \\
\text { Gliclazide, glimepiride, glipizide, } \\
\text { glyburide, glibenclamide }\end{array}$ & Gain & $\begin{array}{l}\text { Increased risk of CV mortality and } \\
\text { all-cause mortality }\end{array}$ & $\begin{array}{l}\text { Hypoglycaemia, } \\
\text { weight gain }\end{array}$ & Second-line drug therapy \\
\hline $\begin{array}{l}\text { TZDs } \\
\text { Rosiglitazone, pioglitazone }\end{array}$ & Gain & $\begin{array}{l}\text { Increased risk of MI, CHF, Increased } \\
\text { levels of LDL-C, triglycerides }\end{array}$ & $\begin{array}{l}\text { Weight gain, } \mathrm{CV} \\
\text { outcomes }\end{array}$ & $\begin{array}{l}\text { To be avoided in patients with } \\
\text { or at risk for heart failure }\end{array}$ \\
\hline $\begin{array}{l}\text { DPP-4 inhibitors } \\
\text { Sitagliptin, saxagliptin, } \\
\text { linagliptin, alogliptin, } \\
\text { tenaligliptin }\end{array}$ & Weight neutral & Neutral effect on CV outcomes & $\begin{array}{l}\text { GI effects, } \\
\text { pancreatitis }\end{array}$ & $\begin{array}{l}\text { Caution to be exercised while } \\
\text { prescribed in patients with } \\
\text { heart failure }\end{array}$ \\
\hline $\begin{array}{l}\text { AGIs } \\
\text { Acarbose, miglitol, voglibose }\end{array}$ & Weight neutral & Neutral effect on CV outcomes & $\begin{array}{l}\text { Flatulence, } \\
\text { diarrhoea }\end{array}$ & $\begin{array}{l}\text { Low risk of hypoglycaemia. } \\
\text { CV safety to be evaluated }\end{array}$ \\
\hline $\begin{array}{l}\text { SGLT2i } \\
\text { Dapagliflozin, canagliflozin, } \\
\text { empagliflozin, remogliflozin }\end{array}$ & Weight loss & Beneficial CV effects & $\begin{array}{l}\text { Mycotic infections } \\
\text { of the genital tract } \\
\text { and osmotic diuresis }\end{array}$ & $\begin{array}{l}\text { Favoured as the second-line } \\
\text { agent of choice in T2DM } \\
\text { patients with a history of CVD }\end{array}$ \\
\hline
\end{tabular}

AGIs: Alpha-glucosidase inhibitors, CHF: Congestive heart failure, CV: Cardiovascular, HDL-C: High-density lipoprotein-cholestrol, DPP-4: Dipeptidyl peptidase, GI: Gastrointestinal, MI: Myocardial infarction, LDL-C: Low-density lipoprotein-cholestrol, SGLT2i: Sodium-glucose co-transporter 2 inhibitors, DM: Diabetes mellitus T2DM: Type 2 DM, TZDs: Thiazolidenediones, SU: Sulfonylureas, CVD: CV disease 
high risk of hypoglycaemia, older patients and patients with CKD. ${ }^{[177]}$ Shorter-acting secretagogues, the meglitinides (or glinides), also stimulate insulin release through similar mechanisms and may be associated with comparatively less hypoglycaemia but they require more frequent dosing. Moreover, modern sulfonylureas exhibit more reductions of $\mathrm{HbA} 1 \mathrm{c}$ than glinides. ${ }^{[178]}$

\section{Thiazolidinediones}

Pioglitazone and rosiglitazone are peroxisome proliferator activated receptor $\gamma$ activators that improve insulin sensitivity by increasing insulin-mediated glucose uptake in skeletal muscle, suppressing hepatic glucose output, and improving the secretory response of insulin in pancreatic $\beta$-cells. ${ }^{[179]}$ The risk of hypoglycaemia is negligible and TZDs may be more durable in their effectiveness than sulfonylureas. ${ }^{[180]}$ TZDs have been constantly under the authority scrutiny for their cardiovascular safety. A meta-analysis considering data from 42 trials and 27,847 patients indicated that treatment with rosiglitazone was associated with an increase in the odds of MI (odds ratio $1.43,95 \%$ CI 1.03 to $1.98, \mathrm{p}=0.03$ ) and a nonsignificant increase in the odds of cardiovascular death (odds ratio $1.64,95 \% \mathrm{CI} 0.98: 2.74, \mathrm{p}=0.06$ ) compared with a control group (active comparator or placebo). ${ }^{[181]}$ The RECORD study evaluated the effect of rosiglitazone (plus metformin or sulfonylurea) in lowering the combined end point of hospitalization or cardiovascular death versus metformin plus sulfonylurea. Although, rosiglitazone did not increase the risk of overall cardiovascular morbidity or mortality it increased the risks of heart failures causing admission to hospital or death when compared to the active controls (HR: 2.10, 95\% CI, 1.35:3.27). ${ }^{[182]}$ Conversely, pioglitazone is known to exert pleotropic effects on cardiovascular event; pioglitazone improves endothelial dysfunction, lowers hypertension, improves dyslipidaemia, and lowers circulating levels of inflammatory cytokines and prothrombotic factors. ${ }^{[183]}$ In the PROactive study, pioglitazone lowered the composite of all-cause mortality, non-fatal myocardial infarction, and stroke in T2DM patients with at risk of macrovascular events along with improvements in $\mathrm{HbA1c}$, triglycerides, LDL, and $\mathrm{HDL}$ levels. However, rate of heart failure was increased. ${ }^{[184]}$ TZDs have demonstrated beneficial effects in attenuating dyslipidaemia commonly observed in patients with chronic T2DM. Rosiglitazone treatment with metformin showed an HDL increase of $13.3 \%$, LDL increase of $18.6 \%$ and a neutral effect on total cholesterol, following 26 weeks of treatment. ${ }^{[185]}$ Furthermore, the IRIS trial was among the 1st studies to document the CV benefits of TZDs in non-diabetic individuals. Pioglitazone improved CV outcomes (recurrent stroke and MI) and prevented the development of T2DM in insulin-resistant, non-diabetic patients with cerebrovascular disease. ${ }^{[186]}$ In a recent post hoc study of the IRIS trial, conducted in prediabetic population, pioglitazone effectively lowered the risk of stroke, MI, acute coronary syndrome, and hospitalization for heart failure. ${ }^{[187]}$ Pioglitazone had been linked with a possible increased risk of bladder cancer, possibly in a dose-and time-dependent manner. ${ }^{[188,189]}$ However data from a retrospective study in India involving 2222 (pioglitazone users, $\mathrm{n}=1111$; pioglitazone non-users, $\mathrm{n}$ =1111) T2DM patients found no evidence of bladder cancer in any of the group, including patients with age $>60$ years, duration of diabetes $>10$ years, and uncontrolled diabetes. ${ }^{[190]}$ Recognized side effects of TZDs include weight gain (3-5 kg), fluid retention leading to oedema, and/or heart failure in predisposed individuals and patients with increased risk of bone fractures. ${ }^{[180,184,190]}$

\section{Dipeptidyl peptidase-IV inhibitors}

Vildagliptin, saxagliptin, gemigliptin, evogliptin sitagliptin, teneligliptin, and linagliptin are incretin enhancers; they enhance circulating concentrations of active GLP-1 and gastric intestinal polypeptide (GIP). ${ }^{[191]}$ These incretins stimulates insulin secretion, suppresses glucagon synthesis, lower hepatic gluconeogenesis, and slow gastric emptying. Their major effect is the regulation of insulin and glucagon secretion; they are weight neutral. ${ }^{[192]}$ DPP-4 inhibitors are efficient in improving glycaemia both as monotherapy and as add-on to metformin, sulfonylurea and TZDs in patients with inadequate glycaemic control. A reduction in $\mathrm{HbA} 1 \mathrm{c}$ levels from baseline of $8.1 \%$ was observed with sitagliptin monotherapy (100 mg:-0.5\%, $200 \mathrm{mg}:-$ $0.6 \%$ ) in 521 patients treated for 18 weeks. Additionally, homeostasis model assessment of beta cell function index, fasting proinsulin-insulin ratio which are the markers of insulin secretion, and beta cell function were also improved significantly. ${ }^{[193]}$ The overall incidence of adverse events with sitagliptin is comparable to other OADs when used as monotherapy or as add-on to existing OADs ${ }^{[194]}$ Adverse effects (AEs) such as constipation, nasopharyngitis, urinary tract infection, myalgia, arthralgia, headache, and dizziness are the commonly reported AEs with the use of these agents. ${ }^{[195]}$ Cardiovascular outcomes trial (CVOT) studies with DPP-4 inhibitors have shown that these agents are safe in patients with established CVD and those at increased risk of CVD except for increased risk of heart failure risk. ${ }^{[196]}$ Results of the SAVOR-TIMI study and EXAMINE Study have reported higher rates of hospitalization for heart failure with saxagliptin and alogliptin, respectively. ${ }^{[197,198]}$ Owing to the increased risk of hospitalization due to heart failure in patients with cardiovascular disease, the US FDA issued a warning, suggesting the associated risk to be a "class-effect" of the DPP-4 inhibitors and issued a warning for their use. ${ }^{[199]}$ However, some landmark studies have been conducted to evaluate relationship between these drugs and the adverse effects. In the CARMELINA study, linagliptin demonstrated a long-term $\mathrm{CV}$ safety profile in patients with $\mathrm{T} 2 \mathrm{D}$, including those with $\mathrm{CV}$ and/or kidney disease and no increased risk of 
hospitalisation for heart failure versus placebo was reported. [200] The CAROLINA study was designed to evaluate the long-term CV safety profile of linagliptin versus glimepiride in patients with early T2D at increased CV risk. Recently released top line results highlight a non-inferiority between linagliptin versus glimepiride in time to first occurrence of CV death, non-fatal MI, or non-fatal stroke (3P-MACE) with a median follow-up of more than 6 years. ${ }^{[201]}$

\section{Sodium-glucose co-transporter 2 inhibitors}

They provide insulin-independent glucose-lowering by blocking glucose reabsorption in the proximal renal tubule. The capacity of tubular cells to reabsorb glucose is reduced by SGLT2 inhibitors leading to increased urinary glucose excretion and consequently, correction of the hyperglycaemia. ${ }^{[202]}$ Dapagliflozin, canagliflozin, empagliflozin, and remogliflozin are the 4 Drug Controller General of India (DCGI) approved agents used in patients with T2DM. ${ }^{[203,204]}$ The EMPA-REG OUTCOME trial evaluated the non-inferior cardiovascular safety of empagliflozin in high-CV-risk T2D patients with an estimated glomerular filtration ate (eGFR) of at least $30 \mathrm{~mL} / \mathrm{min} / 1.73 \mathrm{~m}^{2}$. Empagliflozin reduced the rate of new onset or worsening nephropathy, which were defined as new-onset microalbuminuria, doubling of creatinine, and eGFR $\leq 45 \mathrm{~mL} / \mathrm{min} / 1.73 \mathrm{~m}^{2}$, initiation of renal replacement therapy, and death due to renal disease (hazard ratio [HR]: $0.61,95 \%$ CI: 0.53, 0.70; $<<0.0001) .{ }^{[205]}$ A secondary analysis of CANTA-SU study confirmed the renoprotective effects of canagliflozin. The eGFR decline was significantly slower in patients receiving canagliflozin than in those receiving glimepiride $\left(0.5 \mathrm{~mL} / \mathrm{min} / 1.73 \mathrm{~m}^{2}\right.$ per year with $100 \mathrm{mg}$ daily [95\% CI: $0.0,1.0]$ and $0.9 \mathrm{~mL} / \mathrm{min} / 1.73 \mathrm{~m}^{2}$ per year with $300 \mathrm{mg}$ daily [95\% CI: $0.4,1.4]$, compared with glimepiride $\left(3.3 \mathrm{~mL} / \mathrm{min} / 1.73 \mathrm{~m}^{2}\right.$ per year [95\% CI: $2.8,3.8]){ }^{[206]}$ The CANVAS Program integrated data from two trials involving a total of 10,142 participants with type 2 diabetes and high cardiovascular risk. Treatment with canagliflozin showed a possible benefit with respect to the progression of albuminuria (HR: $0.73 ; 95 \% \mathrm{CI}$ : $0.67,0.79$ ) and the composite outcome of a sustained $40 \%$ reduction in the estimated glomerular filtration rate, the need for renalreplacement therapy, or death from renal causes (HR: 0.60; 95\% CI: 0.47, 0.77). ${ }^{[207]}$ Canagliflozin in combination with metformin significantly improved glycaemic control in patients with T2DM and significant weight loss along with low incidence of hypoglycaemia have been reported. ${ }^{[208]} \mathrm{A}$ recent meta-analysis concluded that SGLT2 inhibitors, as a class, significantly reduce $24-\mathrm{h}$ ambulatory blood pressure further substantiating their favourable cardiovascular profile. ${ }^{[209]}$ The most common AEs involving this class are genital mycotic infections, which are believed to be mild and respond favourably to anti-fungal therapy. ${ }^{\text {210-214] }}$

\section{Alpha glucosidase inhibitors}

These agents delay the absorption of consumed carbohydrates by competitively inhibiting the aglucosidase enzymes at the enterocyte brush border. This inhibition delays the digestion of starch and sucrose and maintains levels of postprandial blood glucose excursions. ${ }^{[215]}$ The action of these agents are independent of insulin action and hence are devoid of hypoglycaemic adverse effects. In the Essen-II Study, conducted in 96 patients, acarbose significantly lowered HbA1c levels when compared with placebo and treatment with acarbose was associated with a weight reduction of $-0.8 \mathrm{~kg} .{ }^{[216]}$ When added to background of metformin, treatment with acarbose led to $\mathrm{HbA} 1 \mathrm{c}$ reduction of $0.7 \% .^{[217]}$ The AGIs have demonstrated an acceptable safety profile with major complaints being of flatulence and diarrhoea.

The glucose-lowering effectiveness of OADs is said to be high with metformin, sulfonylureas, and TZDs (expected HbAlc reduction $\sim 1.0-1.5 \%$ ) and comparatively lower for meglitinides, DPP4 inhibitor, SGLT2 inhibitor, AGIs. ${ }^{[195]}$ However, older drugs have typically been tested in clinical trial participants with higher baseline HbA1c, which is associated with greater treatment emergent glycaemic reductions, irrespective of therapy type. In head-to-head trials, any differential effects on glucose control between different OADs are small. So agent and patient-specific properties, such as ease of administration, dosing frequency, side effect profiles, cost, and other benefits, often help in their selection.

- Two-drug combination therapies with metformin (such as metformin plus TZDs, metformin plus sulfonylureas, metformin plus SGLT2 inhibitors, and metformin plus DPP4 inhibitors, DPP4+ SGLT2) were more effective in reducing $\mathrm{HbA} 1 \mathrm{c}$ than metformin monotherapy by about $1 \% .{ }^{[218]}$ In addition, triple FDC of metformin and sulfonylurea plus pioglitazone are also available in India.

- $\quad$ RSSDI wheel given along with this recommendation book will help a practitioners choose an ideal drug for his patient based on cost, weight, hypoglycaemia risk, and other comorbid conditions. 


\section{Treatment 3: InJectables}

\section{RECOMMENDATIONS}

\section{Recommended Care}

- Insulin therapy should be considered in all patients failing to achieve glycemic targets on three oral agents

- Consider initiating Insulin in type-2 diabetes patients with symptomatic severe hyperglycemia or unstable state.

- A three step protocol involving initiation, titration, and intensification is recommended for all patients requiring insulin.

- Initiation

- "Providers should avoid using insulin as a threat or describing it as a sign of personal failure or punishment and should work to alleviate patient anxiety about hypoglycaemia, dependence, injection-site pain etc., commonly attributed to insulin"

- Therapeutic choice of regimen, preparation, and delivery device, should be made through a process of shared, informed decision making

- Initiate with once-daily basal insulin, once-daily premixed/co-formulation insulin, or twice-daily premixed insulin, either alone or in combination with or other OADs, based upon patient's age, clinical features, glucose profile, risk of hypoglycaemia, and patient preference. Basal insulin may also be initiated in combination with GLP-1 analogues

- Basal bolus insulin regimens may be needed in severe hyperglycaemia, and in life threatening or organ/limb threatening clinical situations.

- Analogue insulins may be used in preference to human insulins with possible lower risk of nocturnal and symptomatic hypoglycaemia; however, economic considerations must be taken into account.

- Match timing of insulin and meals.

- Counselling/education about SMBG and hypoglycaemia prevention, recognition and treatment are recommended to all patients initiating with insulin.

- Provide guidance for adjusting insulin dose adjustments, administration, storage and other practical aspects.

- Titration

- Initiate insulin as defined in the algorithm, using a self-titration regimen (dose increases of 2-4 unit (U) units every three days or biweekly) or with more frequent contact with a healthcare professional

- Aim for pre-meal glucose levels of $<115 \mathrm{mg} / \mathrm{dL}$ and PPG levels of $140-160 \mathrm{mg} / \mathrm{dL}$. These targets can be individualized, based upon the risk of hypoglycaemia and the urgency for glycaemic control

- Titration should be done at regular and short intervals, to attain glycaemic goals without causing hypoglycaemia and as guided by the physician or HCPs

- Titration should be done to control FBG first, followed by prandial control of meal with highest glycaemic excursion in sequential order.

- Intensification

- Intensification of insulin therapy is recommended when patients fail to achieve glycaemic goals even after optimal dose titration.

- Several options can be considered during intensification. In patients on basal insulin-

- Switch to premix insulin twice-daily or premix analogues twice or thrice-daily

- Switch to insulin co-formulation based regimen

- Add prandial insulin (basal plus or basal bolus) with largest meal of the day

- Add GLP-1 analogues

- The choice of intensification strategy should be based upon dietary pattern, lifestyle, risk of hypoglycaemia and weight gain, affordability, as well as patient preference.

- Basal plus regimen can be used as a stepwise approach to insulin intensification, leading to basal-bolus prescription. It is associated with lesser risk of hypoglycaemia and weight gain than basal bolus regimen.

- Both premix insulin therapy and co-formulation insulins are acceptable methods of intensification. Co-formulation insulin offers the advantage of lower risk of hypoglycaemia and nocturnal hypoglycaemia.

- Follow insulin intensification as recommended in the algorithm.

- GLP-1 analogues

- GLP-1 analogues with proven CV benefit should be considered to reduce the risk.

- Viable second-line or third-line options for the management of patients with uncontrolled hyperglycaemia.

- Can be considered in overweight/obese patients as second line therapy in patients with metformin inadequacy and as first line therapy in patients with metformin intolerance.

- To be added to insulin therapy preferably basal insulin only if glycaemia goals are not achieved with reasonably high doses of insulin, or if unacceptable weight gain or hypoglycaemia occurs. Dose reduction of insulin may be needed in such cases. Transient gastrointestinal side effects may occur.

\section{Limited Care}

- All conventional insulins have similar glycaemic lowering efficacy as analogues but with slight increased risk of hypoglycaemia and lack of flexibility of administration.

- Insulin supplies should be assured and be of consistent quality and type. 


\section{BACKGROUND}

Most of treatments available to control glycaemia impact the pathways targeting $\beta$-cells or IR and are dependent upon the presence of insulin for their therapeutic effect. The durability of these medications varies and their safety is occasionally under scrutiny. Over a period, patients fail to achieve or maintain HbAlc levels even with multiple OADs and will require insulin therapy. Although insulin is the most effective option for glycaemic control, it should not be used as firstline treatment in T2DM, as it can predispose hypoglycaemia, weight gain, and large doses over prolonged duration might increase the risk of malignancy and cardiovascular diseases. However, it is equally important to ensure timely initiation of insulin without delay once optimal combinations of oral hypoglycaemic drugs have failed to achieve the target $\mathrm{HbA} 1 \mathrm{c}$.

Most guidelines recommend early short term insulin therapy in patients with high $\mathrm{HbAlc}$ at the time of presentation. ${ }^{[219-221]}$ Landmark trials in last decade suggest that glycaemic control should be intensive in early stages of diabetes, preferably in first four years of diagnosis ${ }^{[222-224]}$ The traditional postponement of insulin therapy up to prolonged failure of lifestyle and oral agents to achieve glycaemic control has been revised in the last decade to incorporate insulin therapy much earlier, often in combination with OADs or GLP-1 analogues. Non-insulin injectables such as GLP-1 analogues and amylin analogues (pramlintide) have been approved in various countries. The GLP-1 analogues improve glycaemic control through multiple mechanisms, with low risk of hypoglycaemia, and clinically relevant weight loss. ${ }^{[225]}$ As pramlintide is not available in India, it will not be covered in these recommendations.

\section{Considerations}

- The decision on choice of injectable therapy in T2DM patients is based on clinical, pharmacological and psychosocial factors [Table 3]. Additionally, local factors such as cost, quality, cold chain maintenance, and perennial availability of insulin preparations as well as delivery devices, must be considered in the Indian context.

- If a study of adequate doses of two to three non-insulin agents for 3-6 months fails to achieve HbA1c targets, or if organ dysfunction contraindicates use of oral agents, addition of insulin may be justified. This idea is supported by the landmark studies suggest that achieving intensive glycaemic control (if not contraindicated) in initial few years of diagnosis is of profound benefit.

- In these cases, insulin may be started as monotherapy or with metformin if the latter is not contraindicated and is well tolerated. As the patient's glucose toxicity resolves, the regimen can potentially be de-escalated, and a switch over to oral therapy may be considered.

- HbAlc targets must be determined as per criteria set for individualized therapy and efficacy of each agent as combination therapy must be considered. ${ }^{[226]}$

- Near-normal glycaemic targets should be considered for younger patients with recent onset of T2DM with few or no micro or macrovascular complications, while slightly higher $\mathrm{HbAc}$ targets may be considered for older patients with long-standing T2DM and evidence of CVD, end organ failure, and terminal illnesses. ${ }^{[227]}$

- While initiating insulin, doses of OADs should modified as following:

- No change in dose of metformin, DPP4i, SGLTi, AGI, TZDs

- Dose of sulphonulurea should be reduced when prandial insulin is introduced.

- Adequate doses of oral agents do not necessarily mean the highest administrable doses because, in most of the cases, doubling the doses of these medicines does not necessarily increment their effects.

\section{Rationale and Evidence}

\section{The insulin strategy}

- While initiating the insulin therapy, the following features have to be considered in a sequential order: choosing the right regimen, identifying the appropriate preparation, prescribing the available strength of the preparation, matching it with the correct delivery device, deciding the proper insulin dose, and following the optimal titration strategy.

- Ideally, an insulin treatment program should be designed specifically for an individual patient, to match the supply of insulin to his or her dietary/exercise habits, and prevailing glucose trends, as revealed through selfmonitoring. Anticipated glucose-lowering effects should be balanced with the convenience of the regimen, in the context of an individual's specific therapy goals.

\section{Patient education}

- Proper patient education regarding monitoring of glucose, insulin injection technique, insulin storage, recognition/

\section{Table 3: Clinical situations where use of injectables is recommended}

\begin{tabular}{lll}
\hline Short term insulin & GLP-1 analogues & Basal insulin + GLP-1 \\
\hline Catabolic symptoms & Recommended as first injectable & $\begin{array}{l}\text { Recommended in } \\
\text { situations where further }\end{array}$ \\
High glycaemic parameters: HbA1c $>9.0 \%, F P G>200$ and $/$ or PPG $>300 \mathrm{mg} / \mathrm{dL}$ & $\begin{array}{l}\text { option in following situations } \\
\text { (standard care): Concomitant }\end{array}$ & $\begin{array}{l}\text { intensification of } \\
\text { Acute diabetic complications/medical conditions }\end{array}$ \\
$\begin{array}{l}\text { Hospitalised patients not suitable for OADs: Perioperative, transplants, critical care units } \\
\text { Pregnancy }\end{array}$ & $\begin{array}{l}\text { CVD, CKD, where hypoglycaemia } \\
\text { and weight loss must be avoided }\end{array}$ &
\end{tabular}

CKD: Chronic kidney disease, CV: Cardiovascular, CVD: CV disease, HbA1c: Glycosylated haemoglobin, OADs: oral antidiabetics, FPG: Fasting plasma glucose, GLP-1: Glucagon-like peptide 1, PPG: Postprandial glucose 
treatment of hypoglycaemia, and sick day management is imperative. Where available, certified diabetes educators can be invaluable in guiding the patient through treatment course of diabetes.

\section{Adverse events and barriers}

- Hypoglycaemia is a major safety concern with insulin treatment and can be a barrier for initiation or intensification. ${ }^{[228]}$

- Addition of Sulphonylurea and TZDs can accentuate the risk of weight gain with insulin treatment ${ }^{[229]}$ and therefore agents which are weight neutral or weight reducing should be combined with insulin where weight gain is a concern. Combination with DPP4 inhibitors resulted in weight neutrality and combination with metformin or AGIs produced weight loss compared to insulin monotherapy. ${ }^{[230]}$

- Various barriers to insulin use prevail in society. A recent National Insulin Summit (NIS) consensus lists the barriers to insulin therapy as related to patient/community, physician/provider, and drug/device and proposes different bridges to overcome these hurdles. Patient-related barriers such as inability to inject, monitor, or titrate the insulin dose, weight gain, hypoglycaemia, and lack of awareness of uncontrolled diabetes can be bridged with patient education and training, support, and counselling, and social marketing. Physician and provider barriers such as inadequate communication or motivation skills, inability to initiate, optimize or intensify insulin, and lack of awareness may be addressed through relevant skill development training, and continuing medical education
(CME). Furthermore, drug or device-specific barriers such as suboptimal effects of insulin, lack of flexibility, and device discomfort can be surmounted through CME, flexible insulin regimens, and preparations and modern devices. ${ }^{[231]}$

\section{Initiation of insulin therapy [Figures 3 and 4]}

- As initial therapy, unless the patient is passing through an acute medical, surgical or obstetric crisis, or in metabolic decompensation, premixed or basal insulin is typically initiated [Tables 5 and 6]. ${ }^{[219,221,232,233]}$ General concept is to first correct the fasting hyperglycaemia with a dinner/bed time injection, and then address postprandial hyperglycaemia.

- Choice of initial insulin is often dictated by subjective features such as disease severity, and ability of the patient to self-inject at specific times of the day [Table 4]. Even though FPG and PPG measurements together provide sufficient information to choose an insulin type, it is difficult to make an appropriate decision when they are considered separately. Similarly, choice of insulin on the basis of HbAlc value alone can be challenging. ${ }^{[234]}$

- All international and Indian guidelines recommend insulin initiation either with a basal or premixed/co-formulation insulins except ADA/EASD and AACE guidelines which prefer basal insulin for initiation.

\section{Intensification of Insulin therapy [Tables 7 and 8]}

- $\quad$ Although most of the patients with T2DM requiring insulin therapy can be successfully treated with one or two doses

Table 4: Choice of insulin therapy

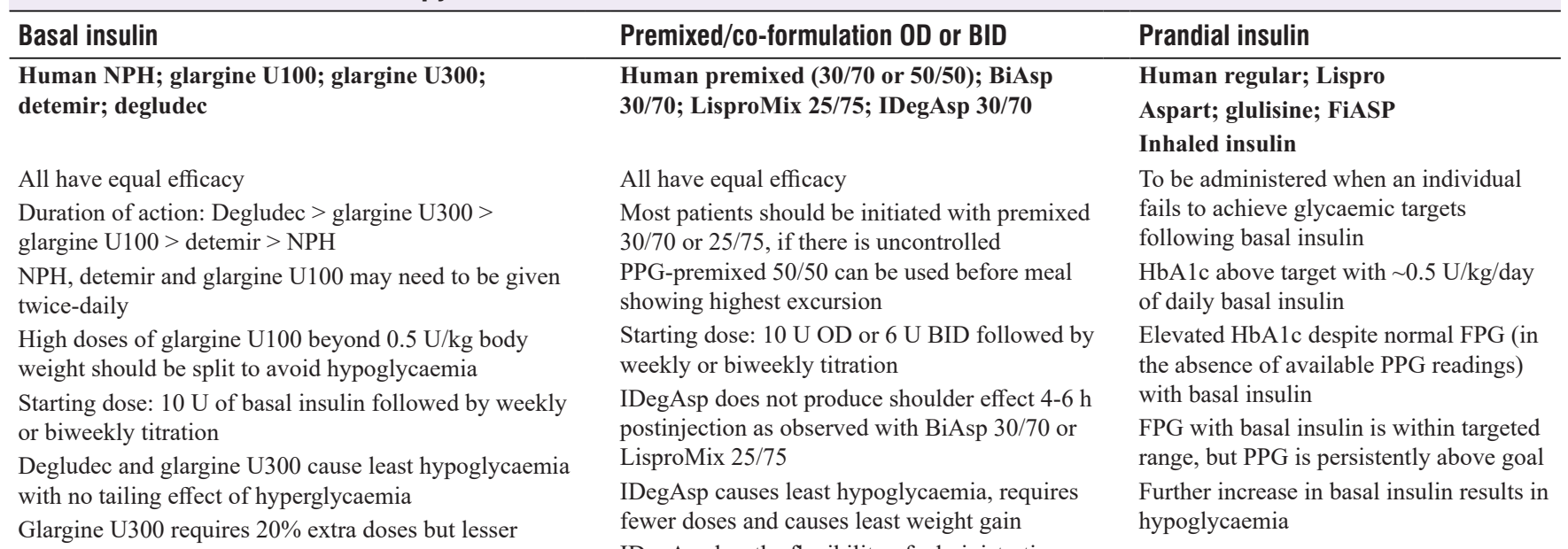

volume is required

Glargine U300 can be used where high volume of insulin is required

Detemir causes least weight gain

Basal insulin should be given preferably at bedtime to

achieve adequate suppression of HGP

$\mathrm{NPH}$ and detemir are approved for use in pregnancy and

IDegAsp has the flexibility of administration before any large meal of the day and can be given with different meals on different days BiAsp 30/70 and LisproMix 25/75 are approved for uses in pregnancy with steroid use

BID: Twice daily, BiAsp: Biphasic insulin aspart, IDegAsp: Mix of insulin degludec and insulin aspart, NPH: Neutral protamine Hagedorn, OD: Once daily, HGP: Hepatic glucose production, FPG: Fasting plasma glucose, HbA1c: Glycated haemoglobin, PPG: Postprandial glucose 


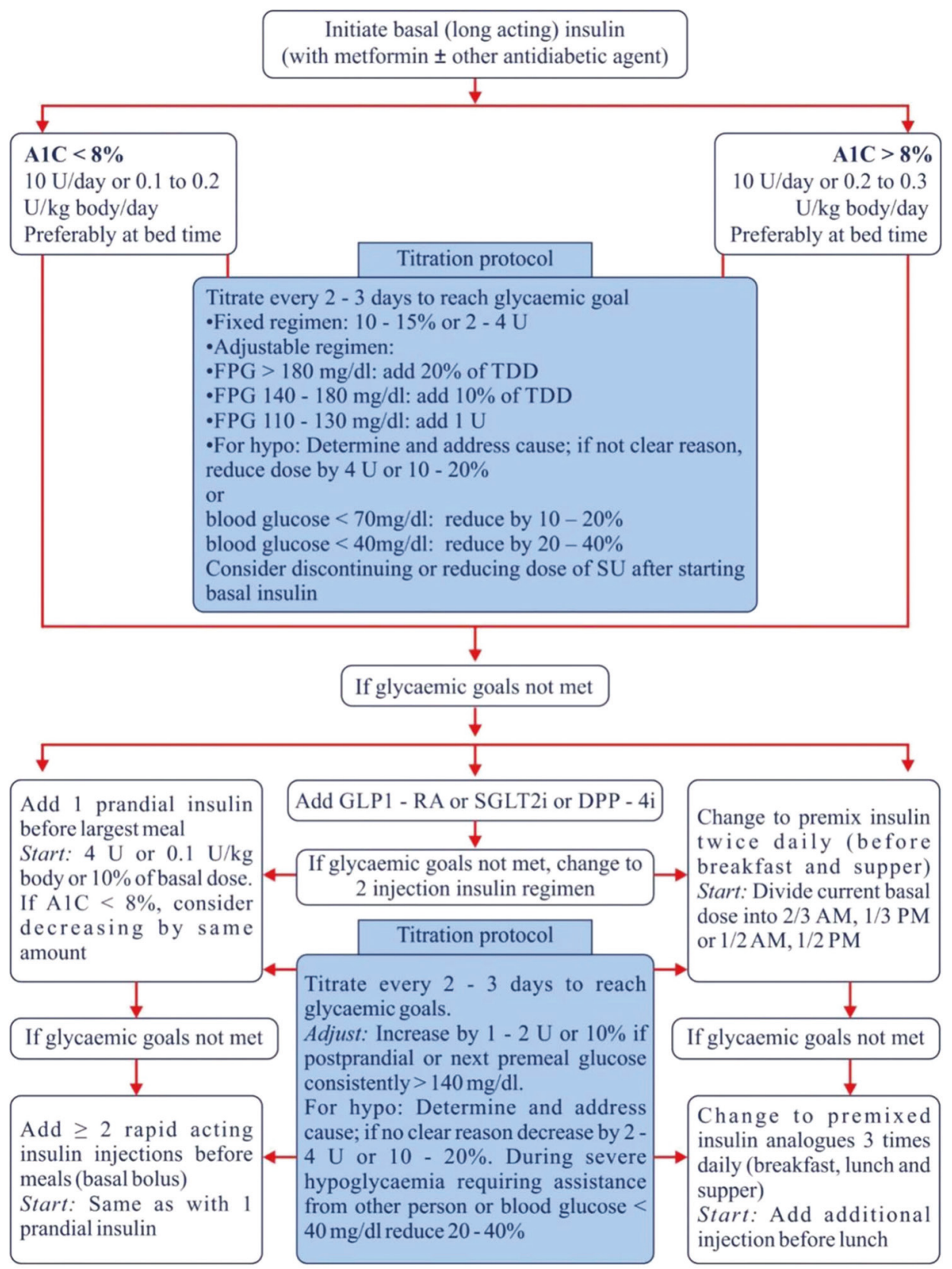

Figure 3: Approaches for initiating insulin. OD: Once daily; BID: Twice daily; TID: Three times a day; GLP-1 RA: Glucagon like peptide-1 receptor agonist; IAsp: Insulin aspart; IDegAsp: Mix of insulin degludec and insulin aspart; A1c: Glycated haemoglobin; DPP-4i: Dipeptidyl peptidase-4 inhibitors; FPG: Fasting plasma glucose; GLP-1 RA: Glucagon like peptide-1 receptor agonist; SGLT2i: Sodium glucose co-transporter 2 inhibitors; TDD: Total daily dose

of insulin, some, because of progressive diminished in their insulin secretory capacity, will require prandial insulin therapy as well. This is typically achieved with regular insulin administered about 30 min before meals or rapid insulin analogues such as insulin lispro (ILis), insulin aspart (IAsp), or insulin glulisine (IGlu), which can be injected just before or with the meal. They result in better PPG control than human regular insulin.

\section{Glucagon-like peptide 1 analogues}

- The injectable GLP-1 analogues like liraglutide, exenatide, lixisenatide, dulaglutide and albiglutide imitate the effects of endogenous GLP-1, thereby stimulating pancreatic insulin secretion in a glucose dependent fashion, suppressing pancreatic glucagon output, slowing gastric emptying, and decreasing appetite. Their main advantage is weight loss, which can be significant in some of the patients. Limiting side effects of these agents are nausea and vomiting, particularly early in the course of treatment. ${ }^{[235]}$

- In combination with basal insulin they have proved to be extremely useful option for intensification because of complimentary action (IDegLira, Lixilan). Tirate as per dose

- There have been concerns regarding an increased risk of pancreatitis with GLP-1 analogues but recently published 


\section{Table 5: Steps for initiating basal insulin}

\begin{tabular}{lcc}
\hline & Glucose value & TDD \\
\hline $\begin{array}{l}\text { Step 1: Initiationwith basal } \\
\text { insulin* }\end{array}$ & HbAlc $<8 \%$ & $0.1-0.2 \mathrm{U} / \mathrm{kg}$ \\
$\begin{array}{l}\text { Step 2: Titration\# (every 2-3 days } \\
\text { to reach glycaemic goals) }\end{array}$ & HbAlc $>8 \%$ & $0.2-0.3 \mathrm{U} / \mathrm{kg}$ \\
& Fixed regimen & Increase by $2 \mathrm{U} / \mathrm{day}$ \\
& Adjustable regimen & Add $4 \mathrm{U}$ \\
Step 3: Monitor for & $\mathrm{FPG}>180 \mathrm{mg} / \mathrm{dL}$ & Add $2 \mathrm{U}$ \\
hypoglycaemia & FPG $140-180 \mathrm{mg} / \mathrm{dL}$ & Add $1 \mathrm{U}$ \\
& FPG $110-139 \mathrm{mg} / \mathrm{dL}$ & Reduce by $10 \%-20 \%$ \\
\end{tabular}

*Consider discontinuing SU therapy and basal analogues should be preferred over NPH insulin, ${ }^{\#}$ For most patients with T2DM taking insulin, glucose goals are $\mathrm{HbAlc}<7 \%$ and fasting and premeal $\mathrm{BG}<110 \mathrm{mg} / \mathrm{dL}$ in the absence of hypoglycaemia. HbA1c and FPG targets may be adjusted based on patients age, duration of diabetes, presence of comorbidities, diabetic complications, and hypoglycaemia risk. DM: Diabetes mellitus, BG: Blood glucose, FPG: Fasting plasma glucose, HbA1c: Glycosylated haemoglobin, NPH: Neutral protamine Hagedorn, SU: Sulfonylureas, T2DM: Type 2 DM, TDD: Total daily dose

\section{Table 6: Titration algorithm}

\section{Basal insulin Prandial insulin}

Given preferably at bedtime to achieve adequate suppression of HGP Target: FPG $<120$

Initiate with $10 \mathrm{U}$ at bedtime and check FBSL

Increase dose by $1 \mathrm{U} /$ day or $3 \mathrm{U}$ every 3 days by patient self-titration till target FBSL is achieved
Initiate along with meal with highest glycaemic excursion

Start with $4 \mathrm{U}$ and increase by $1 \mathrm{U} /$ day or $3 \mathrm{U} / 3$ days till PPG $<180$

Next meal with highest glycaemic excursion should be titrated similarly

Full basal bolus can be considered for effective prandial control after all meals

\section{Premixed insulin}

Calculate the total dose

Start with 6 U BID day for analogues and 2/3 dose in morning and $1 / 3$ dose in evening for human insulins

Titration can be done for morning dose based on predinner values and for evening dose based on FBG

Titration can be done by $1 \mathrm{U} /$ day or $3 \mathrm{U} /$ day to achieve required BG targets

BID: Two times daily, BG: Blood glucose, FBG: Fasting BG, FBSL: Fasting blood sugar level, FPG: Fasting plasma glucose, HGP: Hepatic glucose production, PPG: Postprandial glucose

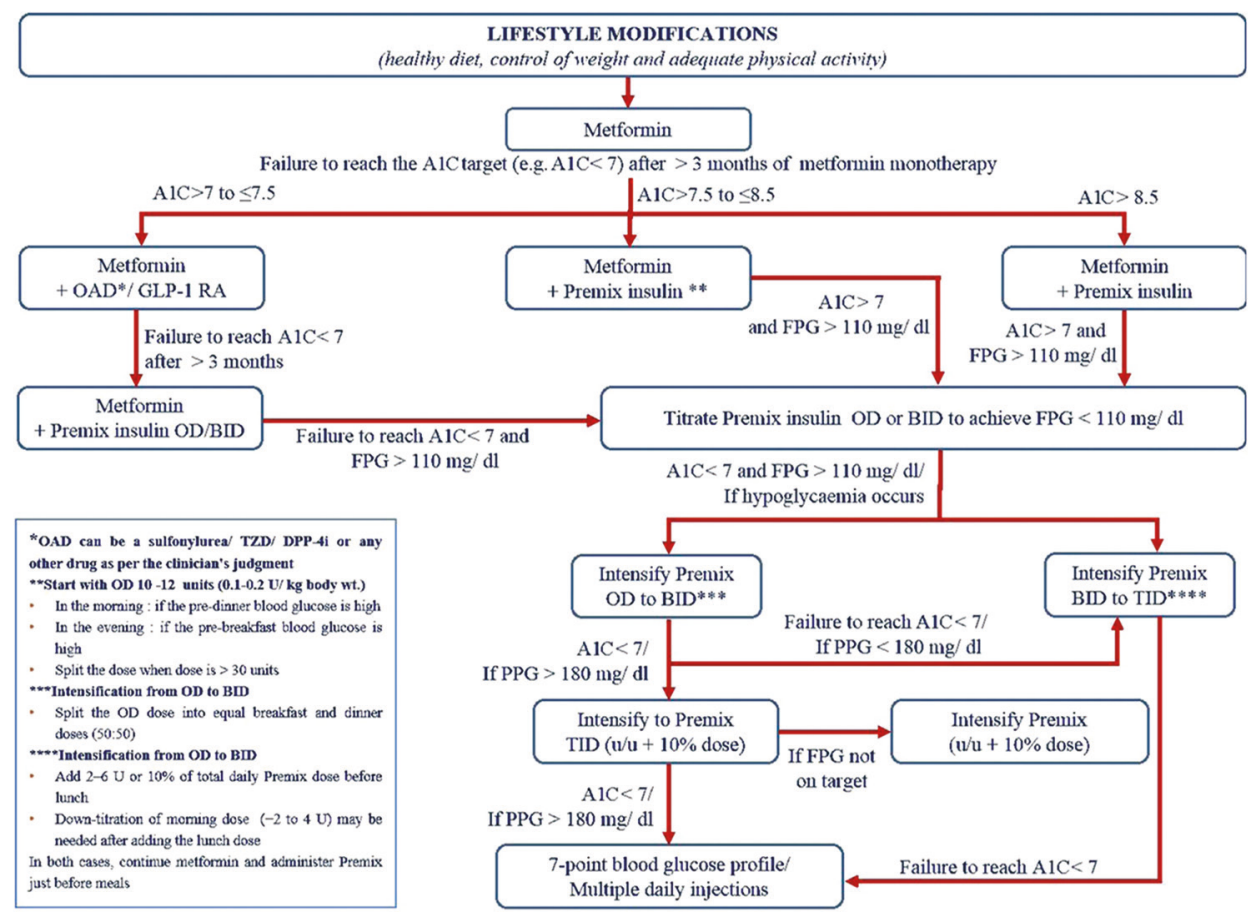

Figure 4: Steps for initiating premixed insulin. OAD: Oral antidiabetic agents; GLP-1 RA: Glucagon like peptide-1 receptor agonist; 0D: Once-daily; BID: Twice-daily; TID: Thrice-daily; TZD: Thiazolidinedione; DPP-4I: Dipeptidyl peptidase-4 inhibitors 
ELIXA and LEADER study do not show any increased risk of pancreatitis, pancreatic cancer, or thyroid cancer with lixisenatide or liraglutide. ${ }^{[236,237]}$

- Furthermore, in the LEADER trial, the primary composite outcome of first occurrence of death from CV causes, non-fatal MI, or non-fatal stroke was significantly less with liraglutide as compared to placebo (HR, 0.87; 95\% CI: $0.78,0.97 ; p<0.001$ for non-inferiority; $p=0.01$ for superiority) ${ }^{[237]}$ On the basis of this result, liraglutide is approved for its CV benefits as well by FDA "as an adjunct to standard treatment of $\mathrm{CV}$ risk factors to reduce the risk of major adverse $\mathrm{CV}$ events (CV death, non-fatal MI, or non-fatal stroke) in adults with T2DM and high CV risk."[238]

\section{IMPLEMENTATION}

Timely initiation and appropriate intensification of injectable therapy is a challenging aspect of diabetes care. This must be addressed by focusing on patient education and motivation, as well as updating knowledge of health care professionals. Lifestyle modification, self-monitoring, and insulin education, should be integral parts of insulin therapy in T2DM. Structured guidelines and protocols should be shared, and glycaemic audits of persons on oral medications performed to address the issue.

\section{Table 7: Insulin intensification options}

\begin{tabular}{|c|c|}
\hline Insulin regimen & Characteristics \\
\hline Basal plus regimen & $\begin{array}{l}\text { One basal insulin along with one prandial insulin before the meal showing largest PPG excursion } \\
\text { Advantages: Flexibility and can be further intensified to cover } 2 \text { or } 3 \text { meals (full basal bolus regimen) } \\
\text { Disadvantages: Needs two insulins, two pens, and two different titrations for individual components bringing in } \\
\text { complexity in the regimen }\end{array}$ \\
\hline $\begin{array}{l}\text { Premixed insulins BID or } \\
\text { TID }\end{array}$ & If used thrice-daily then afternoon dose should be $4-6 \mathrm{U}$ to start with and morning dose to be reduced by $10 \%-20 \%$ \\
\hline Co-formulation IDegAsp & $\begin{array}{l}\text { BID is sufficient and has been found to be as effective as basal bolus regimen } \\
\text { Can be given before any two large meals of the day so long as the interval between two injections is } 6 \mathrm{~h} \\
\text { (corresponding to the time action profile of aspart component) }\end{array}$ \\
\hline & $\begin{array}{l}\text { Compared with Basal plus regimen twice-daily, IDegAsp causes similar efficacy, lesser nocturnal hypoglycaemia } \\
\text { and has the convenience of one insulin and one pen } \\
\text { Compared with basal bolus regimen, IDegAsp causes similar reductions in HbA1c, lesser hypoglycaemia, requires } \\
\text { lesser doses, lesser weight gain, and is simple to administer }\end{array}$ \\
\hline Basal bolus regimen & $\begin{array}{l}\text { Most physiological and most effective regimen for intensification } \\
\text { Requires one or two injections of basal insulin, and } 3 \text { injections of prandial insulins } \\
\text { Complex regimen and requires understanding of different titration schedules for basal and prandial components } \\
\text { Knowledge of carbohydrates counting is desirable for proper dosing of prandial component } \\
\text { Needs very frequent monitoring which could be painful and expensive }\end{array}$ \\
\hline
\end{tabular}

BID: Twice daily, IDegAsp: Mix of insulin degludec and insulin aspart, PPG: Postprandial glucose, TID: Thrice daily, HbA1c: Glycosylated haemoglobin

Table 8: Steps for intensification of insulin therapy

\begin{tabular}{|c|c|c|}
\hline & Therapeutic option & TDD \\
\hline Step 1: Add prandial insulin & When glycaemic targets are unmet & TDD $0.3-0.5 \mathrm{U} / \mathrm{kg}(40 \%-50 \%$ basal: $50 \%-60 \%$ prandial $) *$ \\
\hline \multirow{8}{*}{$\begin{array}{l}\text { Step 2: Titration\# (every 2-3 days } \\
\text { to reach glycaemic goals) }\end{array}$} & Fixed regimen (prandial insulin) & Increase TDD by $2 \mathrm{U} /$ day \\
\hline & Adjustable regimen (prandial insulin) & \\
\hline & $\mathrm{FPG}>180 \mathrm{mg} / \mathrm{dL}$ & Increase TDD by $4 \mathrm{U}$ \\
\hline & FPG $140-180 \mathrm{mg} / \mathrm{dL}$ & Increase TDD by $2 \mathrm{U}$ \\
\hline & FPG $110-139 \mathrm{mg} / \mathrm{dL}$ & Increase TDD by $1 \mathrm{U}$ \\
\hline & 2-h PPG or next premeal glucose $>180 \mathrm{mg} / \mathrm{dL}$ & Increase prandial dose for the next meal by $10 \%$ \\
\hline & Premixed insulin & \\
\hline & FPG/premeal BG $>180 \mathrm{mg} / \mathrm{dL}$ & Increase TDD by $10 \%$ \\
\hline \multirow{3}{*}{$\begin{array}{l}\text { Step 3: Monitor for } \\
\text { hypoglycaemia }\end{array}$} & Fasting hypoglycaemia & Reduce basal insulin dose \\
\hline & Night time hypoglycaemia & $\begin{array}{l}\text { Reduce basal insulin or reduce short/rapid-acting insulin } \\
\text { taken before supper or evening snack }\end{array}$ \\
\hline & Between meal hypoglycaemia & Reduce previous premeal short/rapid acting insulin \\
\hline
\end{tabular}

*Basal + prandial insulin analogues preferred over NPH + regular insulin or premixed insulin, ${ }^{\text {} F o r ~ m o s t ~ p a t i e n t s ~ w i t h ~ T 2 D ~ t a k i n g ~ i n s u l i n, ~ g l u c o s e ~ g o a l s ~}$ are $\mathrm{HbA} 1 \mathrm{c}<7 \%$ and fasting and premeal $\mathrm{BG}<110 \mathrm{mg} / \mathrm{dL}$ in the absence of hypoglycaemia. HbA1c and FPG targets may be adjusted based on patient's age, duration of diabetes, presence of comorbidities, diabetic complications, and hypoglycaemia risk. BG: Blood glucose, FPG: Fasting plasma glucose, NPH: Neutral protamine Hagedorn, 2-h PPG: 2-h postprandial glucose, TDD: Total daily dose, FPG: Fasting plasma glucose, T2D: Type 2 diabetes, HbA1c: Glycosylated haemoglobin 


\section{Treatment 4: Individualized Therapy}

\section{RECOMMENDATIONS}

\section{ABCD (EFGH) approach for diabetes management}

Choice of any OADs agent should take into account the patient's general health status and associated medical disorders. This patient centric approach may be referred to as the ABCD (EFGH) approach for diabetes management. As shown in the Figure 1 and Table 2 for any T2DM patients, first line of therapy should be metformin unless it is not tolerated by the patient or contraindicated.

\section{Individualized treatment}

- For patients who have been diagnosed with diabetes, consider a combination of metformin and one of the treatment options based on patients Age, BMI, CKD, Duration of diabetes, Established CVD, Financial condition, Glycaemic status and Hypoglycaemia concern.

- Drug choice should be based on patient preferences as well as presence of various comorbidities and complications, and drug characteristics, with the goal of reducing blood glucose levels while minimizing side effects, especially hypoglycaemia and weight gain.

- A comparative effectiveness meta-analysis suggests that most of available non-insulin agents added to metformin therapy lowers HbAlc around 0.9-1.1\%. ${ }^{[239]}$ Moreover, current National Insulin Summit (NIS) consensus from India report that all oral antidiabetic agents and GLP-1 agonists can reduce $\mathrm{HbA} 1 \mathrm{c}$ to a range of $0.5-2.0 \%$ and insulin can reduce $\mathrm{HbA} 1 \mathrm{c}$ up to $3.5 \%$ when used as monotherapy. ${ }^{[240]}$

Age

- DPP-4 inhibitors may be a suitable addition to metformin for elderly patients (65 years) to avoid hypoglycaemia and weight gain, however, the dosage should be adjusted as per eGFR. ${ }^{[238]}$ Recent double-blind RCTs have reported that gliptins are efficacious and safe with minimal tolerability issue, when used as an add-on therapy in elderly patients with T2DM. ${ }^{[24-244]}$

- Agents belonging to AGIs could also be important choice in elderly patients. These agents have modest efficacy and do not cause hypoglycaemia, but the major limiting factor for their use is the gastrointestinal side-effects, such as flatulence and diarrhoea. ${ }^{[24]} \mathrm{A}$ double-blind RCT revealed that compared to diet alone, addition of acarbose improved the glycaemic profile and insulin sensitivity in elderly patients with T2DM. ${ }^{[246]}$

- The use of glitazones is restricted in elderly T2DM patients owing to the anticipated complications like weight gain, fluid retention, peripheral oedema, aggravation of congestive heart failure, and osteoporosis in postmenopausal women. Newer SUs like gliclazide MR and low-dose glimepiride (due to low risk of hypoglycaemia) can be safely used in elderly patients with T2DM. ${ }^{[171,247]}$

- Evidence regarding the use of GLP-1 agonists and SGLT2i in elderly T2DM patients has been made recently available. The available data suggests that agents of both the classes provide good glycaemic control in patients with T2DM and can reduces CV risk. However, certain drawbacks such as cost, discomfort of injection with GLP-1 agonists, and increased risk of genital mycotic infections and urinary tract infections, hypovolaemia, postural hypotension and weight loss with SGLT2i may limit their usage in some frail elderly T2DM patients. ${ }^{[47]}$

- Evidence suggests that basal insulin analogues such as glargine, detemir and degludec are effective and safe with less risk of hypoglycaemia and weight gain compared to NPH or Premix insulins. ${ }^{[28,249]}$ Moreover a pooled analysis from RCTs revealed that addition of modern insulin analogues compared to NPH insulin to oral antidiabetic drugs in older adults was effective with regards to lower risk of hypoglycaemia. ${ }^{[250]}$

- Individualization of therapy is highly desirable based on risk of hypoglycaemia, comorbidities, functionality, cost, and personal preference in elderly diabetics.

\section{Body mass index}

- While prescribing pharmacological treatments for overweight or obese patients with T2DM, providers should first consider anti-diabetes medications which cause either weight loss or weight neutrality. GLP1 agonists, SGLT2i are associated with weight loss while DPP-4 inhibitors and AGIs appear to be weight neutral. ${ }^{[251,252]} \mathrm{A}$ systematic review and meta-analysis of 62 randomized trials revealed that, when compared to other antidiabetic agents, SGLT2i and GLP-1 agonists were associated with clinically significant body weight loss (range, $1.15-2.26 \mathrm{~kg}$ ) as add-on to metformin. . $^{[253]}$

- GLP-1 agonists and/or SGLT2i seems to be the best add on therapy for those having high BMI. This group of medications has highest weight reducing property in addition to excellent efficacy. A recent systematic review and mixed treatment comparison meta-analysis report thatGLP-1 agonists are associated with weight loss $(-1.62 \mathrm{~kg}$ to $-1.01 \mathrm{~kg})$ in overweight or obese patients with $\mathrm{T} 2 \mathrm{DM}$ with no difference in weight loss between different types of GLP-1 agonists. ${ }^{[254]}$

- $\quad$ SGLT2i also have a weight reduction property. Evidence suggests that SGLT2i were associated with weight loss in patients with T2DM. ${ }^{[255,256]}$ Agents of this class have an additional advantage of acceptable tolerance and can be given orally. However, a careful consideration should be taken with regards to known side effects such as recurrent genital infections, postural hypotension and dehydration especially in summer seasons

- Gliptins are weight neutral and thus can be used as third line of agents..$^{[257,258]}$ Newer agents such as teneligliptin treatment once daily led to significant and clinically meaningful reductions in HbAlc and PPG in Indian patients with T2DM. ${ }^{[259]}$ 
- Use of newer SUs such as gliclazide MR and glimiperide compared to older SUs and other OADs do not result in significant weight gain in patients with T2DM. ${ }^{[260-262]}$

- The lean patients with T2DM with low-normal body mass index $(<18)$. are a distinct group of patients. These patients are usually younger age at onset, have more prevalent use of insulin, higher prevalence in males and higher rates of cigarette smoking and alcohol abuse. ${ }^{[263]}$

\section{Complications (diabetic kidney disease)}

- In patients with renal impairment, preference of therapy would be gliptins as add on therapy with metformin. Few of the gliptins need dose adjustment as per eGFR; linagliptin and teneligliptindoes not require any dose adjustment in renal disease. ${ }^{[264-267]}$

- Repaglinide is another agent which may be used across all stages of renal insufficiency. Similarly pioglitazones may be used in CKD; however, one has to be careful about fluid retention and $\mathrm{CCF}^{[268,269]}$

- Short acting SUs like glipizide and gliclazide can be preferred in patients with moderate/severe renal impairment. Furthermore, in mild/moderate renal impairment, gliclazide MR and glimepiride can also be used, preferably at lower doses. ${ }^{[171]}$

- Although GLP-1 agonists, especially liraglutide and dulaglutide are recommended up to eGFR $15 \mathrm{ml} / \mathrm{min}$, however, owing to their GI adverse effect, their use in renal insufficiency patients is practically limited. ${ }^{[268]}$

- $\quad$ AGIs can be used in patients with mild to moderate renal disease up to eGFR $>30 \mathrm{ml} / \mathrm{min}^{[269]}$

- Insulin can be used in any stages of renal insufficiency. Insulin analogues are preferred over conventional insulins, ${ }^{[270]}$ however, insulin doses may require reduction with falling eGFR and individualized HbA1c targets. ${ }^{[271,272]}$

- Refer to DKD section for the dose modification for SGLT2 $\mathrm{i}$ in patient s with DKD.

\section{Duration of diabetes}

- Patients with long-standing T2DM are difficult to treat because these patients often lack sufficient $\beta$-cell function to respond to secretagogues. Additionally they may have other comorbidities including renal impairment. ${ }^{[273]}$

- Insulins are often used in patients with long-standing diabetes to address insulinopenic states. ${ }^{[273]}$

- Incretin-based therapies, particularly GLP-1 agonists, also lowers HbA1c significantly and have lower risks of hypoglycaemia than insulin. ${ }^{[273]}$ Longer-acting GLP-1 agonists may be preferred over DPP-4 inhibitors as they are more effective than gliptins.

- $\quad$ SGLT2i may also be useful as second add on agent due to their insulin independent action. ${ }^{[274]}$

- AGI's can be also effective sometime owing to their betacell independent actions.

\section{Established cardiovascular diseases}

- UKPDS-33 have suggested that intensive glycaemic control can reduce cardiovascular risk and microvascular complications in patients with T2DM. ${ }^{[275]}$

- In patients with established CVD, GLP-1 analogues and SGLT2i with proven efficacy may be preferred. ${ }^{[205,207,237,276-280]}$

- In patients with heart failure and CKD, SGLT2i or GLP-1 agonists may be preferred unless contraindicated. ${ }^{[205,207,278,281,282]}$

- Pioglitazone has been shown in few studies to reduce CVD risk, ${ }^{[184,186]}$ however, pioglitazone should not be used in patients with subclinical heart failure ${ }^{[280]}$ or patients with low ejection fraction. ${ }^{[283]}$

- Linagliptin, glimepiride and gliclazide MR can be preferred over conventional sulfonylureas in patients at increased risk of CVD or with CVD. ${ }^{[171,284,285]}$

\section{Financial concern}

- Considering that many Indian patients do not have medical insurance and treatment needs to be continued lifelong, cost of therapy also plays a crucial role in T2DM patients from Indian subcontinent.

- Sulphonylureas can be good partner to metformin considering their cost in the light of recent CAROLINA trial demonstrating the $\mathrm{CV}$ neutrality of glimepiride compared to linagliptin. ${ }^{[284]}$ Pioglitazone or inexpensive DPP-4 inhibitors or SGLT2i can also be considered when combinations of SUs and metformin cannot achieve the desired target. Conventional insulin can be used at any stage considering its efficacy and cost.

\section{Glycaemic status}

- The order of glucose lowering agents according to their efficacy of $\mathrm{HbA} 1 \mathrm{c}$ reduction are insulin, GLP-1 agonists, SGLT2i, pioglitazone, DPP-4 inhibitors, SUs, glinides and AGIs. ${ }^{[240,286-288]}$

\section{Hypoglycaemia concern}

- Hypoglycaemia is an important limiting factor during treatment course of diabetes while targeting a good glycaemic control.

- Insulins, Sulfonylureas and glinides have an increased risk of moderate to severe hypoglycaemia compared with other class of agents in monotherapy. Moreover, sulfonylureas as a second-line agent have a greater risk of moderated-severe hypoglycaemia than DPP-4 inhibitors and SGLT2i. ${ }^{[286]}$

- A meta-analysis reported that SUs (relative risk [RR]: 4.57), and glinides (RR: 7.50), were associated with increased risk of hypoglycaemia, compared to thiazolidinediones (RR: 0.56), AGIs (RR: 0.42), DPP-4 inhibitors (RR: 0.63), and GLP-1 agonists (RR: 0.89). [289]

- While initiating DPP-4 inhibitor on a background of secretagogues such as SUs, the dose of secretagogues needs to be reduced and close monitoring of blood glucose 
is necessary. ${ }^{[290]}$ Similarly, while initiating SGLT2i on a background of insulin or secretagogues, the dose of insulin or secretagogues needs to be reduced. ${ }^{[291]}$

- In patients with history of hypoglycaemia or for those at high risk of hypoglycaemia, GLP-1 agonists or SGLT2i or DPP-4 inhibitors or AGIs or pioglitazone should be considered as first choice with metformin. ${ }^{[292]}$

- Patients prone to hypoglycaemia should not preferably be put on glinides, SUs or insulin, since there are high chances of hypoglycaemia with these agents.

- Patients in whom hypoglycaemia further poses risk include:

- Patients with established cv disease

- Elderly patients

- Patients suffering from CKD with or without retinopathy and cannot perform SMBG without the help of others

- Patients who stay alone, especially in remote areas

- Patients who are having poor longevity

- Patients who are having documented hypoglycaemia unawareness
- Patients who met with severe symptomatic hypoglycaemia requiring hospitalization.

\section{IMPLEMENTATION}

The physical impact of T2DM is well known, and its management has substantial effects on individual and societal health, psychological well-being, and quality of life, as well as economic repercussions. Clinical practice recommendations in diabetes management are tools for healthcare providers that can ultimately improve health across populations; however, for improved outcomes, diabetes care must also be individualized for each patient. There is not a one-size-fits-all treatment pattern for patients with T2DM, and diabetes management planning should be individualized. The ADA also highlights the importance of patient-centred care, defined as care that is respectful of and responsive to individual patient preferences, needs, and values and that ensures that patient values guide all clinical decisions. ${ }^{[293]}$ An individualized therapy for T2DM could serve as a "realworld" approach, providing care that is responsive to individuals" specific and unique needs, preferences, and values, and also helping to combat negative long-term outcomes. 
RSSDI-ESI TheRAPEUTIC WheEL

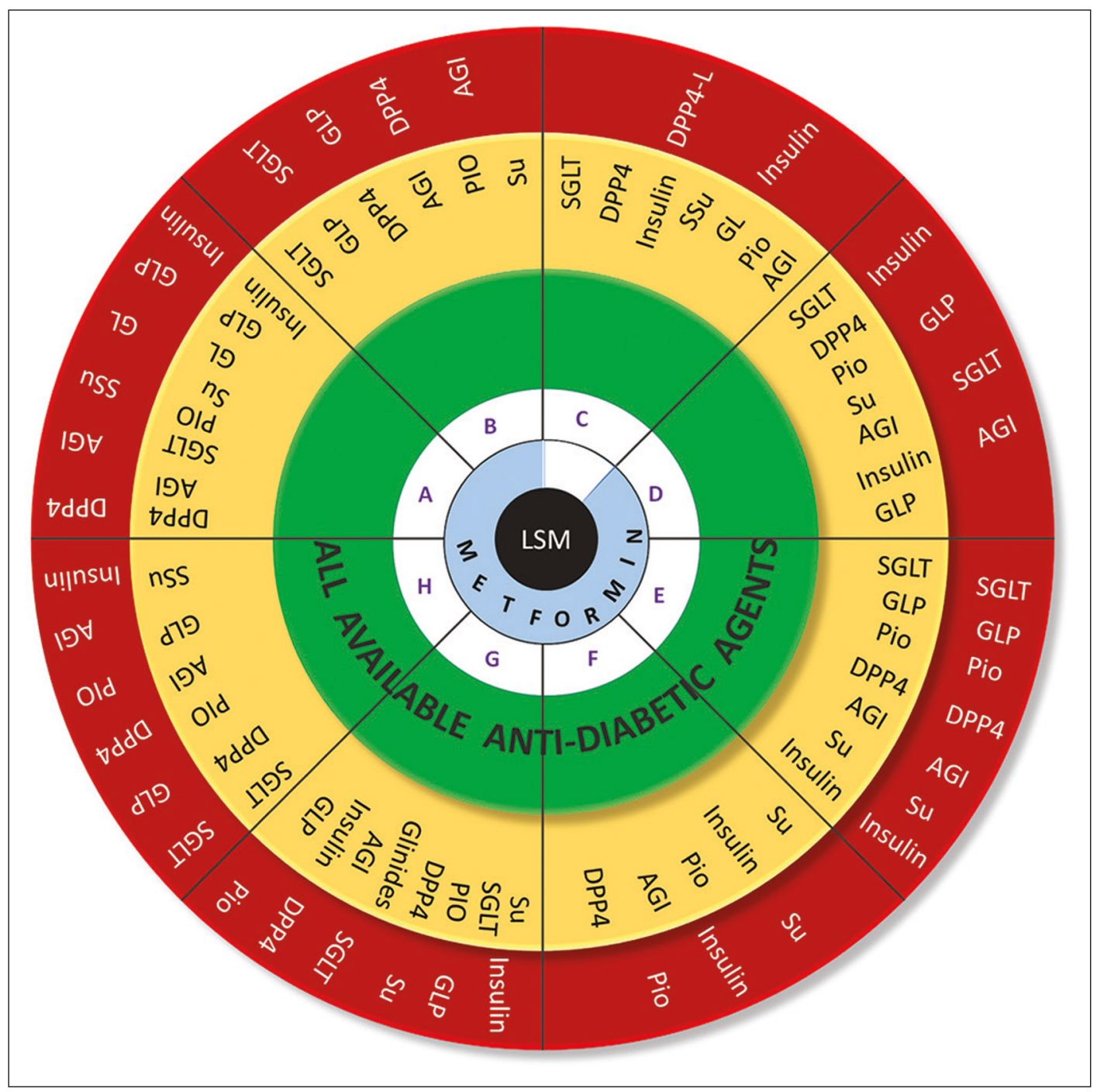

Note: Hydroxychloroquinone is approved by DCGI for the treatment of T2DM as third line therapy

From Innermost to Outermost

$\mathrm{A} \rightarrow$ Age $=$ Advancing age

$\mathrm{B} \rightarrow \mathrm{BMI}=$ Increasing $\mathrm{BMI}$

$\mathrm{C} \rightarrow \mathrm{CKD}=$ Advancing $\mathrm{CKD}$

$\mathrm{D} \rightarrow$ Duration of Diabetes $=$ Increasing duration

$\mathrm{E} \rightarrow$ Established CVD $=$ Low CVD risk to Established CVD Risk

$\mathrm{F} \rightarrow$ Finance $=$ Adequate to Limited

$\mathrm{G} \rightarrow$ Glycemic Status $=$ Worsening glycemic control

$\mathrm{H} \rightarrow$ Hypoglycemia $=$ Hypoglycemia concern

AGI, Alpha-glucosidase inhibitor; DPP4, Dipeptidyl Peptidase-4 (DPP 4) Inhibitors; DPP4-L, Dipeptidyl Peptidase-4 Inhibitors-Linagliptin; GL, Glinides; GLP, Glucagon-like peptide-1 receptor agonist; PIO, Pioglitazone; SGLT, Sodium-glucose Cotransporter 2 Inhibitors; SSu,short acting sulphonylureas; Su, Sulphonylurea; LSM, lifestyle modification

Note: Hierarchy of therapy is depicted in clock-wise manner

GLPS must be used based on costs. Any of the drugs can be used in the green. For other zones, drugs must be used in the given order. 


\section{Postprandial HyPerglycaemia}

\section{ReCOMMENDATIONS}

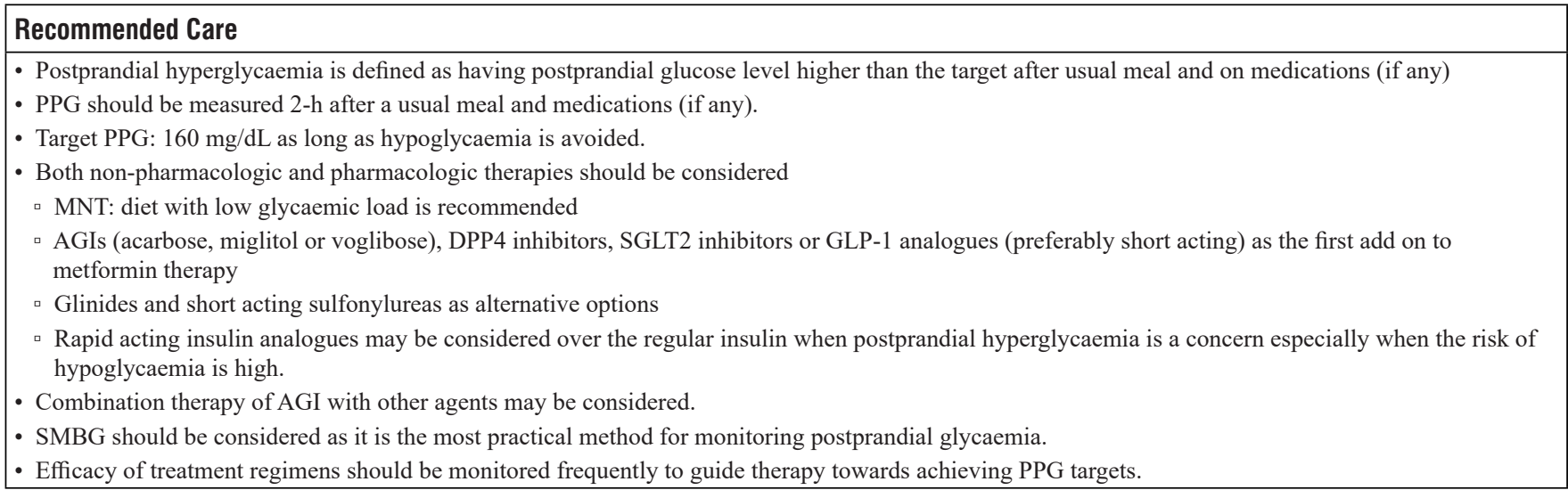

\section{BACKGROUND}

Patients with poorly controlled diabetes frequently develop micro-and macro-vascular complications and evidence from large controlled clinical studies suggest that intensive glycaemic control can significantly reduce their risk of development and/or progression.. ${ }^{[168,222,294-296]}$ Until recently, the predominant focus of diabetes treatment has been on lowering HbAlc levels, with emphasis on FPG. ${ }^{[297,298]}$ However, control of fasting hyperglycaemia alone is insufficient to obtain optimal glycaemic control as evidence suggests that reducing PPG excursion is as important, or perhaps more important for achieving desired glycaemic targets. ${ }^{[299]}$ It is understood that HbAlc is largely impacted by FPG when it is away from target. As it comes closer to the target, PPG starts taking upper hand and contributes predominantly. Therefore, patients who achieved 2-h PPG within the reference limit will better accomplish target $\mathrm{HbA} 1 \mathrm{c}$ values than patients realized FPG within recommended range. ${ }^{[300]}$ In Indians, the PPG remains relatively high all across the $\mathrm{HbA} 1 \mathrm{c}$ spectrum and very high even at higher HbA1c values. ${ }^{[301-303]}$ Relative contribution of postprandial hyperglycaemia to HbAlc levels in patients with T2DM is higher than FPG levels when HbA1c is $<7.5 \%$ and it decreases progressively as HbAlc levels increase. ${ }^{[304]}$ Therefore targeting both PPG and FPG is an ideal strategy for achieving optimal glycaemic control. The purpose of these recommendations is to assist clinicians in developing strategies to consider and effectively manage post-meal glucose in people with T2DM in Asian countries.

\section{Considerations}

India has a high prevalence of diabetes and onset of diabetes is a decade early. Postprandial hyperglycaemia is more prominent in Indians due to high traditional diets with high glycaemic index. Literature is limited regarding postprandial hyperglycaemia despite its definite role in micro-and macrovascular complications.

\section{Rationale and Evidence}

\section{Definition of postprandial hyperglycaemia}

- ADA 2019 and the IDF 2018 define postprandial hyperglycaemia as a 2-h plasma glucose level of $>200 \mathrm{mg} / \mathrm{dL}(11.1 \mathrm{mmol} / \mathrm{L})$ during an oral glucose tolerance test (OGTT). It recommends the use of glucose load equivalent to $75 \mathrm{~g}$ anhydrous glucose dissolved in water as prescribed by $\mathrm{WHO} .^{[1,238]}$

- Asian Indians displayed a marked rise in prandial glucose excursion after consumption of $75 \mathrm{~g}$ of bread meal compared to their Caucasian counterparts. ${ }^{[305,306]}$

- Elevations in PPG are due to decreased first-phase insulin secretion, reduced insulin sensitivity in peripheral tissues, and consequent decreased suppression of hepatic glucose output after meals, unsuppressed glucagon levels and deficiency of intestinal incretin hormones GLP-1 and (glucose-dependent insulinotropic polypeptide) GIP. ${ }^{[297]}$

- The causes of postprandial hyperglycaemia are influenced by many factors which include a rapid flux of glucose from the gut, impaired insulin release, endogenous glucose production by the liver and peripheral IR. ${ }^{[309]}$

- Recent evidences suggest that the value of glycaemia at 1-h during an OGTT is a stronger predictor for developing diabetes than the value at $2-\mathrm{h}^{\left[{ }^{[310-312]}\right.}$ Therefore, in clinical practice, targeting PPG at 1-h instead of 2-h could have significant impact on reducing 


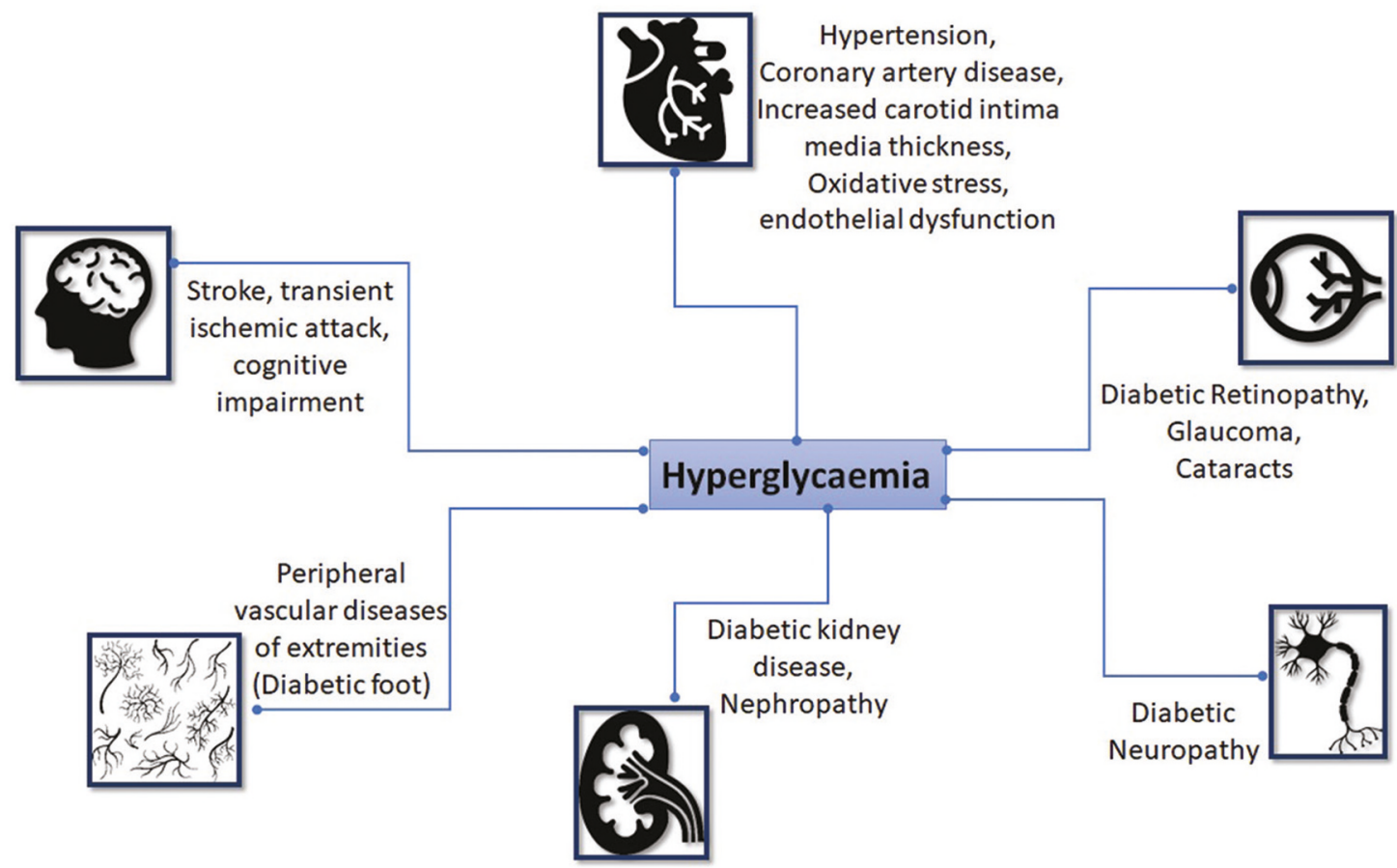

Figure 5: Secondary complications of postprandial hyperglycaemia ${ }^{[307,308]}$

the risk for CVD. The 1-h PPG has been correlated with increased left ventricular mass, left ventricular diastolic dysfunction, and carotid intima-media thickness (CIMT). ${ }^{[313-316]}$

- Evidence from an Indian study based on patients with a history of T2DM for more than 25 years, suggests that postprandial hyperglycaemia was associated with increased risk of both diabetic nephropathy and neuropathy. ${ }^{[301,317]}$ and Kumamoto study suggested reductions in retinopathy and nephropathy with reduced PPG [Figure 5]. ${ }^{[296,318]}$

\section{Addressing postprandial hyperglycaemia}

- Currently, there is lack of data linking improved clinical outcomes with that of correcting postprandial hyperglycaemia. The HEART2D (Hyperglycaemia and Its Effect After Acute Myocardial Infarction on Cardiovascular Outcomes in Patients With Type 2 Diabetes Mellitus) and the NAVIGATOR (Nateglinide and Valsartan in Impaired Glucose Tolerance Outcomes Research) study could demonstrate direct benefit of lowering postprandial hyperglycaemia in reducing CVD in patients with T2DM. ${ }^{[319-321]}$

- However, emerging evidence indicates that agents that target PPG show significant positive trends in risk reduction for all selected CV events. Findings from the Study to Prevent Non-Insulin-Dependent Diabetes Mellitus (STOP-NIDDM) trial highlight that treating people with IGT with acarbose was associated

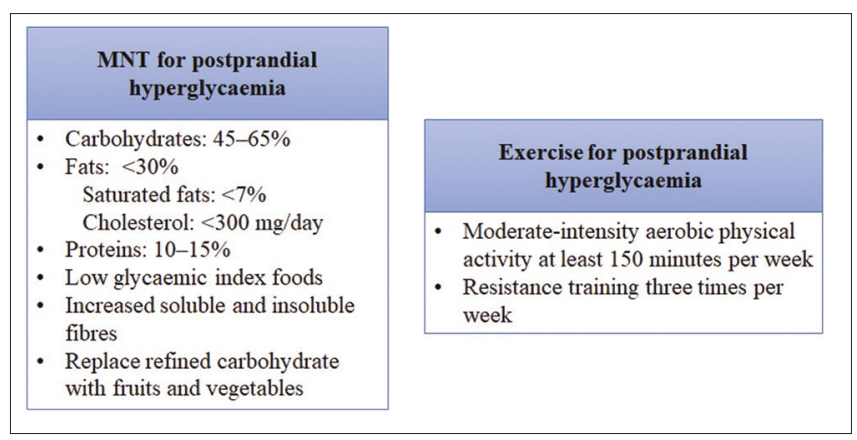

Figure 6: MNT to prevent postprandial hyperglycaemia. MNT: Medical nutrition therapy

with a significant reduction in the risk of CVD and hypertension. ${ }^{[322]}$ The Acarbose Cardiovascular Evaluation (ACE) trial, highlighted that there was no significant impact of acarbose therapy in reducing the risk of major $\mathrm{CV}$ events. However, the incidence of diabetes was reduced, which may mitigate cardiovascular risk in the longer term by delaying the onset of T2DM in the high-risk population. ${ }^{[323]}$

- Postprandial hyperglycaemia is an important pathophysiological state contributing to the several secondary complications including $\mathrm{CV}$ events. Management of postprandial hyperglycaemia is central to long-term glycaemic control and an essential part of CVD prevention in IGT and T2DM. Therefore, it should be routinely monitored in T2DM patients using 2-h post- 
OGTT. Thus, screening for prediabetes and monitoring the glycaemic control in patients with T2DM should include PPG as a predictive marker for all-cause premature death, $\mathrm{CV}$ risks, along with FPG and HbA1c levels. ${ }^{[324-326]}$ The level of implementation of routine screening for post-meal hyperglycaemia, using the OGTT, should be improved in the Asia-Pacific region, combined with wider use of effective interventions to manage postprandial hyperglycaemia. ${ }^{[327]}$

\section{Strategies to prevent postprandial hyperglycaemia Non-pharmacological}

- Physical activity and MNT [Figure 6] are the cornerstones of non-pharmacologic therapy in T2DM patients. ${ }^{[318]}$

- A randomized crossover study showed that in T2DM patients, walking after meals is more effective for lowering postprandial glycaemia. ${ }^{[328]}$

- Traditional Asian Indian and Chinese diets are carbohydrate-rich (as high as $80 \%$ of the macronutrient composition) with high glycaemic index values. ${ }^{[329]}$ Consumption of rice is very high in South India which is associated with 4-5 fold increase in risk of diabetes. ${ }^{[107]}$ The higher carbohydrate load in the Indian diet leads to greater PPG excursion, increased glucosidase and incretin activity in the gut, which leads to higher lipaemic peaks and associated CVD. ${ }^{[329]}$ Evidence suggests that diets with low glycaemic index values are beneficial in controlling postprandial hyperglycaemia. ${ }^{[297,330,331]}$ For details please refer to the MNT and lifestyle section

\section{Pharmacological}

- Based on limited Indian evidence available from literature, the panel relied on expert opinion for pharmacological management of postprandial hyperglycaemia which includes the following:

- GLP-1 analogues are effective in controlling postprandial glucose either when used in association with metformin or part of a combination therapy including basal insulin. The short acting GLP-1 agonists (exenatide and lixisenatide) are preferred when isolated postprandial hyperglycaemia is present.

- The ultrafast acting insulin analogue (FiAsp) has demonstrated significant benefits in reducing 1-h PPG following mealtime administration.

- DPP-4 inhibitors have shown significant benefits in reducing PPG excursions and lowering HbAlc.

- Use of glinides is limited to the treatment of postprandial hyperglycaemia only if sulfonylureas are contraindicated or economic consideration prohibits the use of newer and expensive agents.

- AGIs (acarbose, miglitol, and voglibose) can be used as first-line drug in early T2DM, as well as in combination with nearly all established OADs and insulin. Moreover, AGIs tend to inhibit carbohydrate absorption from gut which can be of particular importance in Indian settings where there are increased odds for PPG and lipid excursion due to consumption of diets with high glycaemic index.

\section{IMPLEMENTATION}

Frequent monitoring of glucose levels using techniques such as SMBG can significantly improve glycaemic control besides detecting PPG excursion. SMBG is currently the optimal method for assessing plasma glucose levels and evidence suggests that structured SMBG followed by therapeutic interventions result in greater $\mathrm{HbA} 1 \mathrm{c}$ reduction in individuals with T2DM compared with programs without structured SMBG ${ }^{[332-334]}$ Therefore, the panel suggest to include SMBG with appropriate patient education for optimal management of post-meal hyperglycaemia. Although, SMBG estimates the average glucose accurately, it underestimates the glucose excursions. Continuous glucose monitoring system (CGMS) provides information on glucose levels, patterns and trends, thereby reflecting the effects of medication, meals, stress, exercise and other factors that affect glucose levels. The CGMS could also be a useful method to detect postprandial hyperglycaemia and to improve therapeutics management in patients with T2DM. ${ }^{[303,335-337]}$ 


\section{Acute Metabolic Complications}

\section{Hyperglycemic Crisis (Diabetic Ketoacidosis and Hyperosmolar Hyperglycemic State)}

\section{RECOMMENDATIONS}

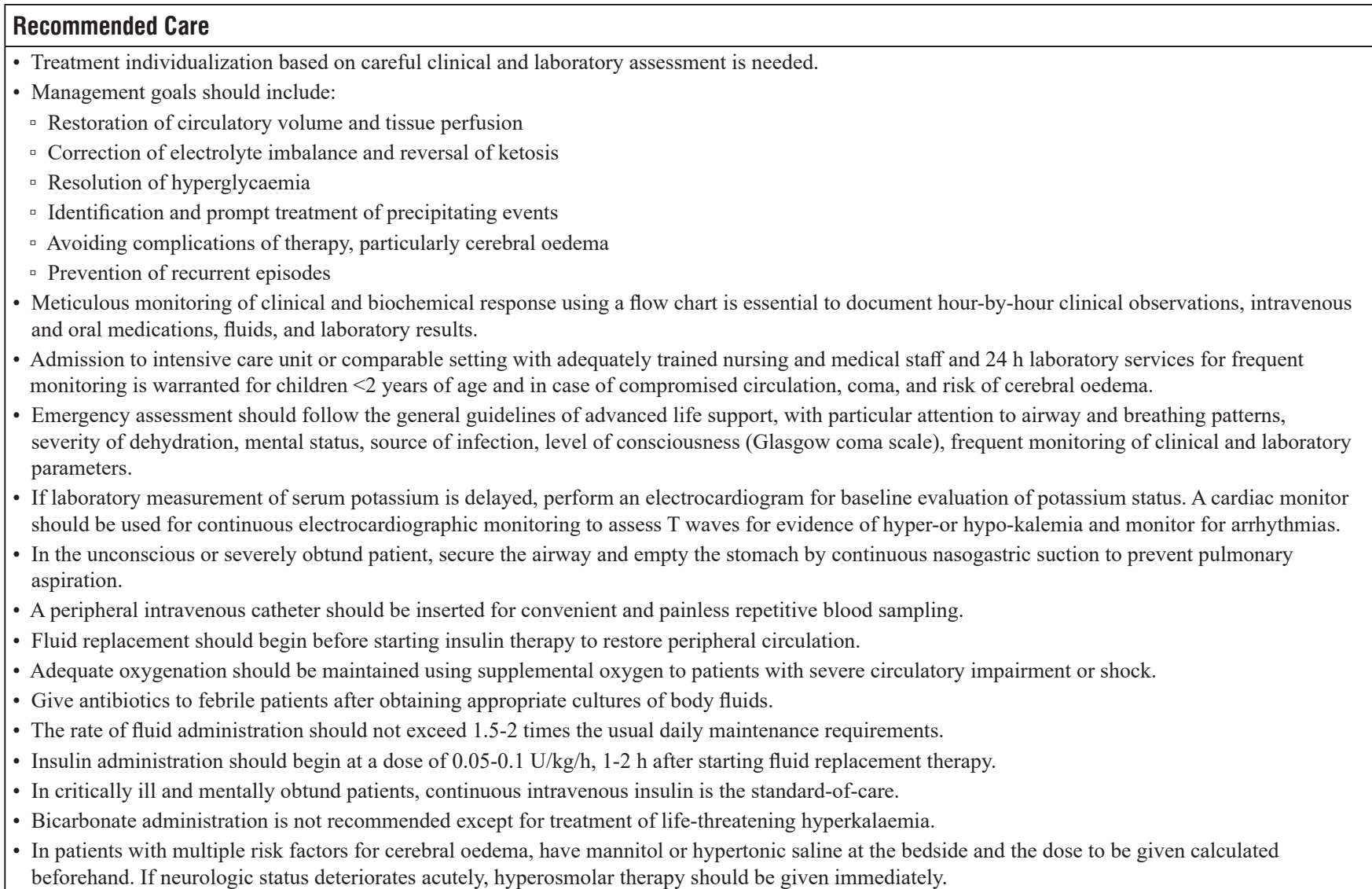

\section{BACKGROUND}

Diabetic ketoacidosis (DKA) and hyperosmolar hyperglycaemic state (HHS) represent the most common and serious acute metabolic complications of diabetes. Both DKA and HHS are associated with substantial morbidity and mortality despite well-developed diagnostic criteria and treatment protocols. ${ }^{[338]}$ The overall DKA mortality recorded is $<1 \%$, but a higher rate is reported among patients aged $>60$ years and individuals with concomitant life-threatening illnesses. ${ }^{[338-340]}$ HHS typically occurs in older patients with T2DM with an intercurrent illness such as infection, surgery or ischemic events, and is associated with higher mortality rate than DKA. Mortality rate with HHS is almost 10 -fold higher when compared with DKA. ${ }^{[341-343]}$

Pathogenesis of these life-threatening hyperglycaemic emergencies is related to absolute or relative insulin deficiency and increase in insulin counter regulatory hormones that lead to an altered metabolism of carbohydrate, protein, and fat and varying degrees of osmotic diuresis and
Table 9: Diagnostic criteria for diabetic ketoacidosis and hyperglycaemic hyperosmolar state

\begin{tabular}{lcc}
\hline Measure & DKA & HHS \\
\hline Plasma glucose level $(\mathrm{mg} / \mathrm{dL})$ & $>250$ & $>600$ \\
Arterial or venous $\mathrm{pH}$ & $<7.30$ & $>7.30$ \\
Serum bicarbonate level $(\mathrm{mmol} / \mathrm{L})$ & $<15$ & $>15$ \\
Urine or blood ketone & Positive & Negative or low \\
Urine or blood $\beta$ hydroxybutyrate $(\mathrm{mmol} / \mathrm{L})$ & $\geq 3$ & $<3$ \\
Effective serum osmolality $(\mathrm{mOsm} / \mathrm{kg})$ & Variable & $>320$ \\
Anion gap $^{\mathrm{a}}(\mathrm{mmol} / \mathrm{L})$ & $>12$ & $<12$
\end{tabular}

${ }^{a}$ Nitroprusside reaction method, ${ }^{b}$ Defined as $2\left[\right.$ measured $\left.\mathrm{Na}^{+}(\mathrm{mEq} / \mathrm{L})\right]$ + glucose $(\mathrm{mg} / \mathrm{dL}) / 18,{ }^{c}$ Anion gap: $\left(\mathrm{Na}^{+}\right)-\left[\left(\mathrm{Cl}^{-}+\mathrm{HCO}_{3}-(\mathrm{mEq} / \mathrm{L})\right]\right.$. DKA: Diabetic ketoacidosis, HHS: Hyperglycaemic hyperosmolar state

dehydration, ketosis, and acidosis. ${ }^{[344]}$ Termed together as decompensated diabetes, the prevalence and mortality for DKA and HHS remains indistinct across various age, gender, and racial groups of hospitalized diabetics. If not interrupted by exogenous insulin, fluid and electrolyte therapy, it would 
lead to fatal dehydration, hypoperfusion, and ultimately metabolic acidosis.

In DKA, insulin deficiency and ketoacidosis are the prominent features of the clinical presentation, and insulin therapy is the cornerstone of therapy [Tables 9 and 10]. Severe hyperglycaemia, osmotic diuresis and dehydration with altered mental status in the absence of significant acidosis characterize HHS. Fluid replacement remains the cornerstone of therapy for HHS. A significant overlap has been reported in more than one-third of patients who exhibit mixed features of both DKA and HHS [Table 11]. Because the three-pronged approach to therapy for either DKA or HHS consists of fluid administration, intravenous insulin infusion, and electrolyte replacement, and mixed cases are managed using the same approach. ICU admission is indicated in the management of DKA, HHS, and mixed cases in the presence of cardiovascular instability, inability to protect the airway, obtundation, the presence of acute abdominal signs or symptoms suggestive of acute gastric dilatation, or if there is not adequate capacity on the floor unit to administer the intravenous insulin infusion and to provide the frequent and necessary monitoring that must accompany its use [Figure 7 and Table 11].

\section{Considerations}

Treatment of patients with DKA and HHS is associated with substantial mortality and healthcare costs. In a developing country like India, due to poor socio-economic status, many patients with T2DM tend to have poor compliance and poor glycaemic control, thus any precipitating factor tends to land them in a state of hyperglycaemic emergencies including DKA and HHS.

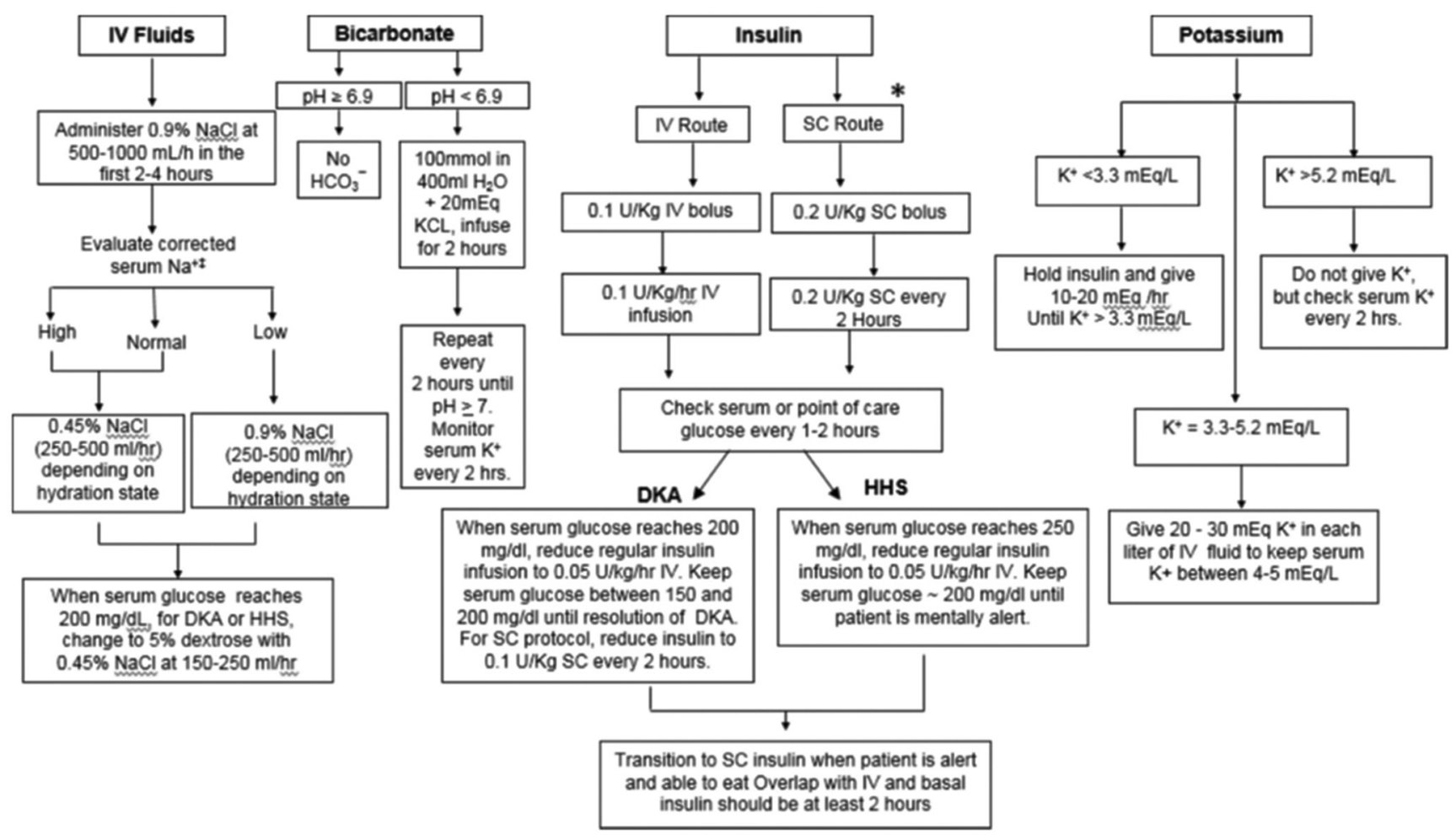

Figure 7: Algorithm for the management of DKA and HHS. DKA: Diabetic ketoacidosis; HHS: Hyperglycaemic hyperosmolar state; IV: Intravenous; SC: Subcutaneous

Table 10: Monitoring of clinical signs and biochemical investigations

\begin{tabular}{ll}
\hline Plasma glucose and HbA1c levels & Venous $\mathbf{p H}$ \\
\hline $\begin{array}{l}\text { BUN, creatinine, electrolytes (including bicarbonates) } \\
\text { with calculated anion gap, and hematocrit }\end{array}$ & Fluid input and output \\
$\begin{array}{l}\text { Serum osmolality } \\
\text { Serum and urinary ketones }\end{array}$ & Vital signs (heart rate, respiratory rate, BP) \\
Arterial blood gases & Neurological observations for warning signs and symptoms of cerebral oedema \\
\hline
\end{tabular}

USG: Ultrasound, BUN: Blood urea nitrogen, ECG: Electrocardiogram, BP: Blood pressure, HbA1c: Glycosylated haemoglobin 


\section{Table 11: Management of acute metabolic complications}

\section{Fluid replacement therapy}

In the absence of cardiac compromise, isotonic saline $(0.9 \% \mathrm{NaCl})$ is infused at a rate of $15-20 \mathrm{~mL} / \mathrm{kg} / \mathrm{h}$ or $1-21$ over $1-2 \mathrm{~h}$ for prompt recovery of hypotension and/or hypoperfusion

Continue with $0.9 \% \mathrm{NaCl}$ at a similar rate if patient is hyponatraemic or switch to $0.45 \% \mathrm{NaCl}$ infused at $250-500 \mathrm{~mL} / \mathrm{h}$ if the corrected serum sodium is normal (eunatraemia) or elevated (hypernatraemia)

When plasma glucose level is $\sim 200 \mathrm{mg} / \mathrm{dL}$, change to $5 \%$ dextrose in saline

Insulin therapy

Start insulin infusion 1-2 $\mathrm{h}$ after starting fluid replacement therapy (after initial volume expansion) and serum potassium restored to $>3.3 \mathrm{mEq} / 1$

Regular human insulin IV bolus of $0.1-0.15 \mathrm{U} / \mathrm{kg}$ followed by continuous insulin infusion at $0.1 \mathrm{U} / \mathrm{kg} / \mathrm{h}$

IV bolus is avoided in children as it may increase the risk of cerebral oedema and can exacerbate hypokalaemia

When glucose level reaches $200 \mathrm{mg} / \mathrm{dL}$ in DKA or $300 \mathrm{mg} / \mathrm{dLin}$ HHS, reduce insulin rate to $0.02-0.05 \mathrm{U} / \mathrm{kg} / \mathrm{h}$. Thereafter, adjust rate to maintain glucose level $150-200 \mathrm{mg} / \mathrm{dL}$ in DKA and 250-300 mg/dL in HHS

Continue insulin infusion until resolution of ketoacidosis

Subcutaneous rapid-acting insulin analogues (lispro and aspart) every 1-2 h. might be an alternative to IV insulin in patients with mildtomoderate DKA Initial dose subcutaneous: $0.3 \mathrm{U} / \mathrm{Kg}$, followed $1 \mathrm{~h}$ later at $0.1 \mathrm{U} / \mathrm{Kg}$ every $1 \mathrm{~h}$, or $0.15-0.2 \mathrm{U} / \mathrm{kg}$ every $2 \mathrm{~h}$

Potassium replacement

If patient is hypokalaemic, start potassium replacement at the time of initial volume expansion and before starting insulin therapy. Otherwise, start after initial volume expansion and concurrent with insulin therapy

With initial rapid volume expansion, a concentration of $20 \mathrm{mmol} / \mathrm{l}$ should be used

The maximum recommended rate is $0.5 \mathrm{mmol} / \mathrm{kg} / \mathrm{h}$

The treatment goal is to maintain serum potassium levels of $4-5 \mathrm{mEq} / 1$

Bicarbonate therapy

Not routinely recommended; only indicated in adults with severe acidosis with $\mathrm{pH}<6.9$

If $\mathrm{pH}<6.9$, consider $100 \mathrm{mmol}$ ( 2 ampules) in $400 \mathrm{ml}$ sterile water with $20 \mathrm{mEq} \mathrm{KCI}$ administered at a rate of $200 \mathrm{ml} / \mathrm{h}$ for $2 \mathrm{~h}$.until $\mathrm{pH}$ is $\geq 7.0$.

If the $\mathrm{pH}$ is still $<7.0$ after this is infused, we recommend repeating infusion every $2 \mathrm{~h}$. until $\mathrm{pH}$ reaches $>7.0$

Transition to subcutaneous insulin

To prevent recurrence of ketoacidosis or rebound hyperglycaemia, consider overlap of IV insulin for 15-30 min (with rapid-acting insulin) or 1-2 h (with regular insulin) or longer (with intermediate or long-acting insulin) after subcutaneous insulin is given

Most convenient time to change to subcutaneous insulin is just before a mealtime

For patients treated with insulin before admission, restart previous insulin

Regimen and adjust dosage as needed

For patients with newly diagnosed DM, start total daily insulin dose at $0.5-0.8 \mathrm{U} / \mathrm{kg} /$ day. Consider multi-dose insulin given as basal and prandial regimen DKA: Diabetic ketoacidosis, HHS: Hyperosmolar hyperglycaemic state, IV: Intravenous, DM: Diabetes mellitus

\section{HyPOGLYCAEMIA}

\section{RECOMMENDATIONS}

\section{Recommended Care}

- Risk of hypoglycaemia should be assessed in every visit in patients with T2DM by using questionnaires.

- Patient should be well educated and informed regarding:

- The symptoms, causes, and risks associated with hypoglycaemia

- Usage of SMBG tools with frequent monitoring especially patients taking insulin

- Insulin dose adjustment considering blood glucose values

- A strict monitoring of hypoglycaemic episodes is recommended for patients taking insulin, sulfonylureas or meglitinides alone or in combination.

- Modern insulins or modern sulfonylureas should be used instead of respective traditional drugs in patients with high risk of hypoglycaemia.

- Oral glucose (15-20 g) is preferred in conscious hypoglycaemic patients (glucose alert value of $<70 \mathrm{mg} / \mathrm{dL}$ ). Repeat the treatment, if SMBG shows continued hypoglycaemia after $15 \mathrm{~min}$. Patient should consume a meal or snack once SMBG returns to normal, to prevent recurrence of hypoglycaemia

- Intramuscular glucagon or intravenous glucose is preferred for unconscious patients or patients with clinically significant hypoglycaemia (glucose alert value of $<54 \mathrm{mg} / \mathrm{dL}$ ). Repeat intramuscular or subcutaneous glucagon dose of $0.5 \mathrm{mg}$ if there is no symptomatic improvement.

- Glucagon to be avoided in patients with sulfonylurea induced hypoglycaemia.

- Treatment should be modified in the event of hypoglycaemia occurring repeatedly at a particular time of the day or in the event of hypoglycaemia unawareness.

- Hypoglycaemia occurring in the setting of advanced kidney disease (CKD stage 4 or 5) requires relatively longer observation for avoidance of recurrence even long after initial corrective measure are taken. 


\section{Limited Care}

- All patients with risk of hypoglycaemia should be enquired about symptomatic and asymptomatic hypoglycaemia at each visit.

- Patients along with their family members should be well educated about identification and management of hypoglycaemia, especially night-time hypoglycaemia.

- Hypoglycaemia should be strictly managed and monitored in special situations such as elderly, pregnancy, fasting, and metabolic disorders.

\section{BACKGROUND}

Hypoglycaemia is a major cause of concern with some antidiabetic drugs during the course of glycaemic management in patients with T2DM. ${ }^{[345]}$ However, the extent of hypoglycaemia varies with different antidiabetic drugs pertaining to their pharmacokinetic and pharmacodynamics properties. The International Hypoglycaemia Study Group categorizes hypoglycaemia into three categories basing upon the glycaemic criteria. ${ }^{[346]}$

- Glucose alert value (level 1): $<70 \mathrm{mg} / \mathrm{dL}(3.9 \mathrm{mmol} / \mathrm{L}$ )

- Clinically significant hypoglycaemia (level 2$):<54 \mathrm{mg} / \mathrm{dL}$ $(3.0 \mathrm{mmol} / \mathrm{L})$

- Severe hypoglycaemia (level 3): no specific glucose threshold.

The prevalence of hypoglycaemia in patients with T2DM in India is quite high. A recent cross-sectional study reports that nearly $96 \%$ of patients (out of 366 patients) were associated with at least one or other symptoms of hypoglycaemia (dizziness, weakness). Furthermore, patients taking insulin in addition to OADs were at higher risk than patient taking OADs alone (OR, 2.3; p <0.01). ${ }^{[347]}$ Meanwhile, another cross-sectional study including 1650 patients from South India revealed that the cumulative incidence of institutional hypoglycaemia was $12.36 \%$; among which, $26.96 \%$ had asymptomatic episodes. ${ }^{[348]}$ Severe hypoglycaemia can lead to several diabetes-related short-and long-term complications such as neurocognitive dysfunction, retinal cell death, and loss of vision ${ }^{[349]}$ and may lead to coma or death if not reversed. ${ }^{\left[{ }^{[35]}\right.}$ The ACCORD and ADVANCE trials and other evidences reported that severe hypoglycaemia was directly associated with mortality in patients with T2DM. ${ }^{[350-352]}$ Furthermore, Kalra et al. stated that diabetes patients with severe hypoglycaemia are associated with sixfold increase in deaths over those not experiencing it. ${ }^{\left[{ }^{[39]}\right.}$ Therefore, urgent steps need to be taken with some corrective measures against hypoglycaemia in T2DM patients to minimize the burden. Following are some of the causes and risk factors of hypoglycaemia [Table 12]. ${ }^{[349]}$

\section{Considerations}

Several factors such as the intensity of hypoglycaemic risk, patient characteristics, drug usage, fasting, and patient education should be considered during framing the recommendations for hypoglycaemia management in patients with T2DM.

\begin{tabular}{ll}
\hline Table 12: Causes and risk factors for hypoglycaemia ${ }^{[347,353]}$ \\
\hline Causes & Risk factors \\
\hline Metabolic defects & Glucose-lowering drugs (especially SU/insulin) \\
Autoimmune conditions & Increased glucose utilization or decreased \\
Dietary toxins & glucose production \\
Alcohol consumption & Female gender \\
Stress & Inborn errors of metabolism \\
Infections & Sleep \\
Starvation & Long duration of diabetes \\
Severe excessive & Extremes of age \\
exercise & Progressive insulin deficiency \\
& Intensive glycaemic control on OADs and \\
& insulin (alone or in combination) \\
& Skipping of meals \\
& DKD, hepatic impairment \\
& Cortisol Insufficiency \\
& Autonomic Failure \\
& Cognitive impairment \\
& Polypharmacy \\
& ACE and $\beta$-blockers
\end{tabular}

ACE: Angiotensin-converting-enzyme, DKD: Diabetic kidney disease, OADs: Oral antidiabetics, SU: Sulfonylureas

\section{Rationale and Evidence}

\section{Identification}

- Symptoms of hypoglycaemia include, but are not limited to, excess sweating and hunger, dizziness, blackout, fainting, fatigue, light-headedness or shakiness, nausea or vomiting, mental confusion or unresponsiveness, and dry-ness or tingling lips. ${ }^{[345]}$

- Nocturnal hypoglycaemia to be suspected in case of night-time sweating, hunger and anxiety. Nightmares, early morning headaches and labile morning sugars should also alert the physician. ${ }^{[354,355]}$

- Some endocrinologists or diabetologists use a threestep approach (Whipple's Triad) for diagnosis of hypoglycaemia. It includes:

- Low blood glucose level

- Symptoms of hypoglycaemia at the time of the low glucose level

- Symptom relief with treatment of hypoglycaemia.

\section{Management}

- Management of hypoglycaemia can be subdivided into three aspects:

- Prevention of hypoglycaemia

- Treatment of hypoglycaemia

- Adjustment or withdrawal or modification of current antidiabetic regimen. 


\section{Prevention of hypoglycaemia}

- Prevention of hypoglycaemia is preferable than treatment, as it is much more likely to avoid severe events and economic burden. ${ }^{[356]}$ Hypoglycaemia prevention requires a combined effort from physician as well as patient. Patient education, patient counselling, and continuous blood glucose monitoring are the critical factors that need to be considered for the prevention of hypoglycaemia in patients with diabetes. Evidence suggests that a proper and structured diabetes education helps in reducing diabetic complications including hypoglycaemia. ${ }^{[357-360]}$

- Furthermore, interventions targeting health beliefs and attitudes about hypoglycaemia and diabetes selfmanagement can be more effective than knowledgecentred patient education, which focuses on symptom perception in reducing hypoglycaemia unawareness. ${ }^{[349]}$ Patients receiving insulin for the treatment of T2DM can be benefitted by adjusting insulin doses following SMBG procedure ${ }^{[349,361]}$ In addition, a cross-sectional study from India reports that $85 \%$ of patients were taking timely meals to prevent hypoglycaemia. ${ }^{[347]}$ Stratifying patients according to age and avoiding very tight glucose control in elderly patients ( $>70$ years) and very young children $<5$ years of age will help to prevent hypoglycaemia in these high-risk people.

\section{Adjustment or withdrawal or modification of ongoing antidiabetic regimen}

- Majority of the antidiabetic agents can produce hypoglycaemia; however, the intensity depends upon their mechanism of action. Insulin, sulfonylureas, and meglitinides due to their glucose-independent mechanism of action cause a high risk of hypoglycaemia. ${ }^{[349]}$

- The UK Hypoglycaemia Study Group report that the incidence of severe hypoglycaemia increased from $7 \%$ to $25 \%$ in patients treated with insulin for $<2$ years with those treated for $>5$ years. ${ }^{[362]}$ However, modern insulin analogues report lower incidence of hypoglycaemia than traditional human insulins. ${ }^{[363-365]}$
- Among all sulfonylureas, modern sulfonylureas like gliclazide MR and glimepiride are associated with lesser hypoglycaemic episodes. ${ }^{[366,367]}$ Meglitinides were reported to inflict high rates of hypoglycaemia. ${ }^{[289]}$ In special situations like elderly, fasting, metabolic disorders, and pregnancy, the dose of these drugs should be adjusted or modified to avoid further complications. Furthermore, avoid/reduce the dose of insulin in people with CKD who have a tendency to develop hypoglycaemia.

\section{Treatment of hypoglycaemia}

- Fifteen to $20 \mathrm{~g}$ of carbohydrate (four teaspoons of sugar or glucose) can be given orally to a conscious patient with hypoglycaemia; if unconscious, glucagon injection intramuscularly or glucose injection intravenously can be preferred. ${ }^{[345,349]}$

- Glucagon to be avoided in sulfonylurea induced hypoglycaemia.

- Caretakers of hypoglycaemia-prone diabetes patients (family members, roommates, school personnel, childcare providers, correctional institution staff, or coworkers) should be well instructed on the use of glucagon kits including where the kit is located and when and how to administer glucagon. ${ }^{[345]}$

- Acute glycaemic response correlates better with the glucose content than with the carbohydrate content of food. Therefore, pure glucose is the preferred treatment. ${ }^{[345]}$ Fifteen minutes after glucose administration, an SMBG should be done and the treatment should be repeated if hypoglycaemia persists. Patient should be advised to eat a regular meal or have a snack to prevent recurrence of hypoglycaemia. ${ }^{[368]}$

\section{IMPLEMENTATION}

Patient empowerment with hypoglycaemia monitoring tools, hypoglycaemia risk awareness, and the available preventive strategies together with physician-patient collaboration plan of treatment can reduce the frequency and intensity of hypoglycaemia.

\section{Chronic Complications 1: Retinopathy, Neuropathy, Diabetic Kidney Disease}

\section{Retinopathy}

\section{ReCOMMENDATIONS}

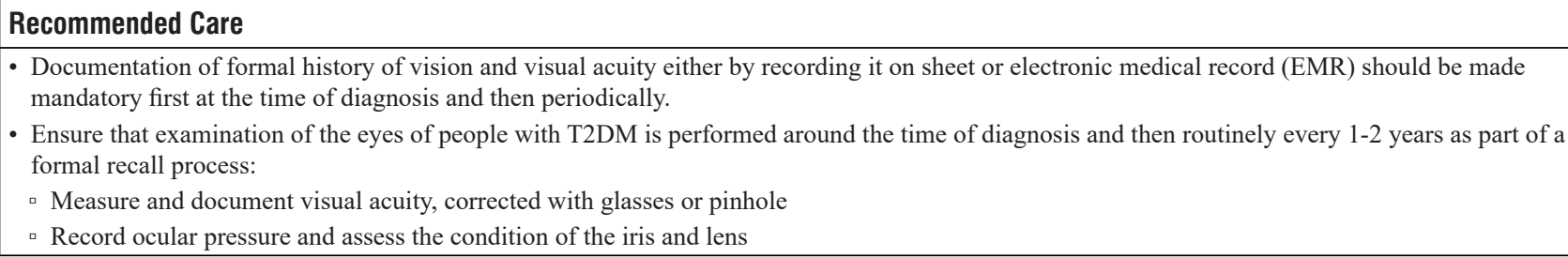

- Documentation of formal history of vision and visual acuity either by recording it on sheet or electronic medical record (EMR) should be made mandatory first at the time of diagnosis and then periodically.

- Ensure that examination of the eyes of people with T2DM is performed around the time of diagnosis and then routinely every $1-2$ years as part of a formal recall process:

- Measure and document visual acuity, corrected with glasses or pinhole

- Record ocular pressure and assess the condition of the iris and lens 


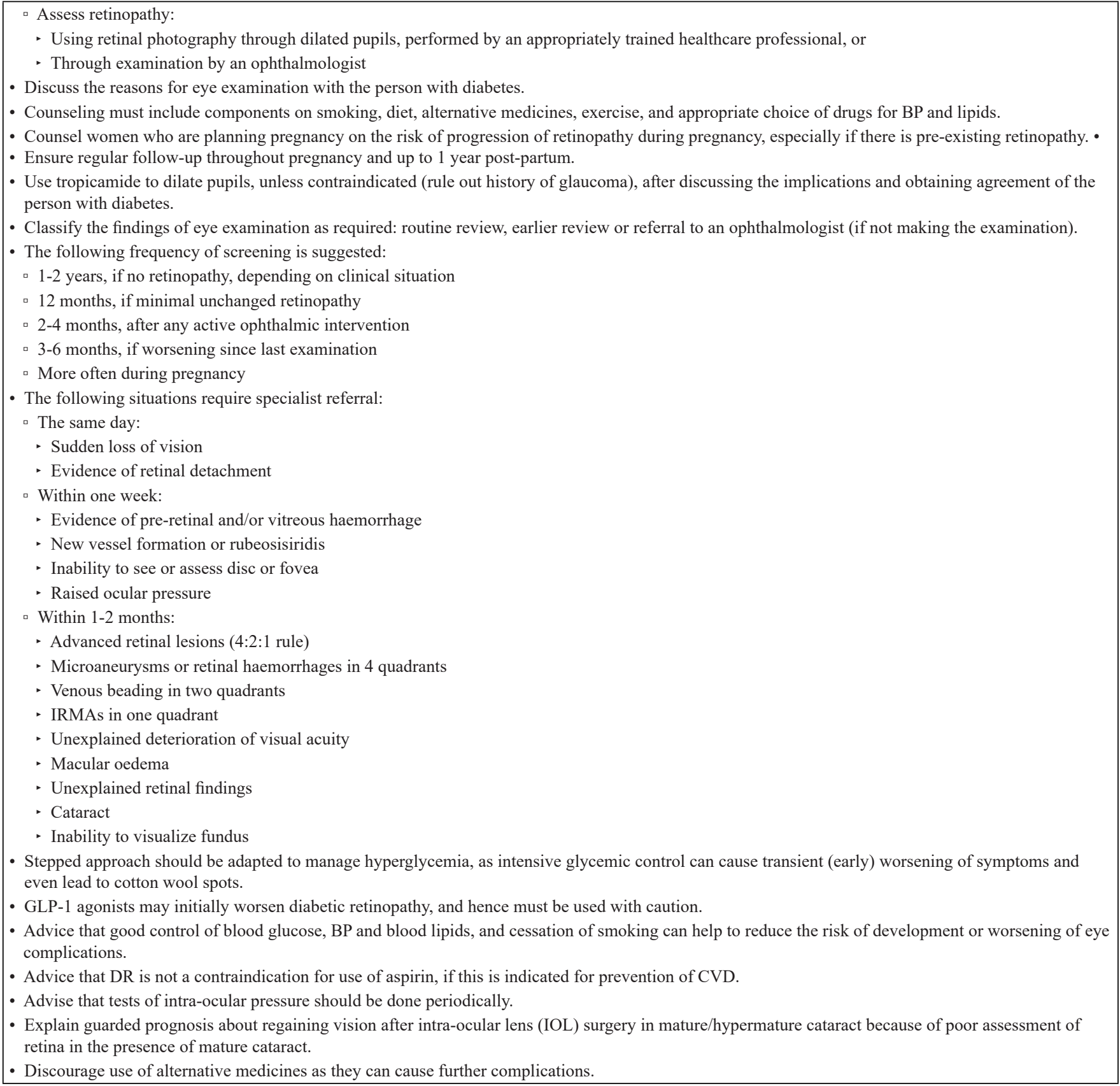

\section{Limited Care}

- Use direct fundoscopy through dilated pupils, performed by a member of the health-care team who is properly trained and has appropriate experience to assess retinopathy.

- Check visual acuity.

- Repeat review, referral and preventative therapy are as for recommended care.

- Less-frequent examinations (every two years) may be considered following one or more normal eye examinations.

- Discourage use of alternative medicines as they can cause further complications.

\section{BACKGROUND}

Diabetic eye disease comprises of a group of eye conditions that affect people with diabetes such as diabetic retinopathy (DR), diabetic macular edema (DME), cataract, and glaucoma.
DR is a microvascular complication of diabetes and one of the leading causes of blindness or vision impairment in India. ${ }^{[369,370]}$ It affects retinal blood vessels and is the most common cause of vision loss among people with diabetes and the leading cause 
of vision impairment and blindness among working-age adults. Visual loss from DR could be due to diabetic macular edema (DME: swelling in the retinal macula) or proliferative diabetic retinopathy (PDR). Factors such as longer duration of diabetes and poorer glycaemic and BP control were found to be strongly associated with DR. ${ }^{[371,372]}$ In a cross-sectional study carried out by All India Ophthalmological Society, prevalence of DR was $21.7 \%$ and the rate was high in men $(\mathrm{p}=0.007)$, in patients with diabetes duration $>5$ years $(p=0.001)$, in patients with age $>40$ years $(p=0.01)$, in insulin users $(p=0.001)$, and in patients with history of vascular accidents $(p=0.0014){ }^{[369]}$ Furthermore, in the cross-sectional survey of Indian patients with T2DM in CINDI (chronic complications in newly diagnosed patients with type 2 diabetes mellitus in India) and CINDI2 (cardiovascular risk factors, micro and macrovascular complications at diagnosis in patients with young onset type 2 diabetes in India), DR was prevalent in $6.1 \%$ and $5.1 \%$ patients, respectively. ${ }^{[373,374]}$ Moreover, the socioeconomic burden resulting from DR induced visual impairment or blindness, particularly in the working age group, is a serious concern. ${ }^{[375]}$ Therefore it is high time to devise the means of managing DR and bring the problem under control. ${ }^{[376]}$ A systematic approach to health education and creating awareness among patients and various health personnel and matching it with appropriate screening and service delivery mechanisms will go a long way in. Early detection and management of DR with quick referrals and a highly coordinated teamwork between the endocrinologists, ophthalmologists, neurologist and nephrologist could reduce the prevalence of DR in India. ${ }^{[377]}$ Important therapeutic measures in the management of DR include optimum glycaemic levels, lipid, and hypertension control. In severe cases of preproliferative DR, laser panretinal photocoagulation (PRP) is indicated with the aim of preventing progression of DR and vision loss. ${ }^{[378]}$ Of note, Implementation of intensive glycaemic control can cause transient (early) worsening, mostly due to the development of small arteriolar infarcts which result in the well-known cotton wool spots, particularly in patients with poor control and long-standing disease are at risk. There for a calculated and stepped approach should be adapted to manage hyperglycaemia is patients with DR. ${ }^{[379]}$

\section{Rationale and Evidence}

\section{Screening}

- Several guidelines emphasize on eye screening in T2DM, however, it appears they are divided on the frequency of screening. Some recommend annual screening (NICEUK) while others recommend screening every 1-2 years (Canadian-Canada, Australian-Australia and SIGN-Scotland).

- With regard to frequency of screening in limited care setting, the panel endorsed the ADA recommendation which suggests less-frequent examinations (every 2-3 years) following one or more normal eye examinations. ${ }^{[380]}$

- $\quad$ Screening methods for DR include direct and indirect ophthalmoscopy, slit-lamp biomicroscopy, stereoscopic colour film fundus photography, mydriatic or nonmydriatic digital colour, and monochromatic photography. ${ }^{[381,382]}$
- In-person clinical exam by an eye care provider is the gold standard for diagnosing DR, faster digital retinal imaging acquisition and grading of DR using fundus images obtained with a nonmydriatic fundus camera is now being considered an effective, cost-sparing, and feasible screening tool for the early detection of DR, and preventing blindness. ${ }^{[383]}$

\section{Counselling pregnant women}

- $\quad$ DR is the foremost cause of blindness in women during the antenatal period, and pregnancy increases the short-term risk of DR progression. ${ }^{[382]}$ The possible relationship between DR and the perinatal outcome has been addressed in several studies. ${ }^{[384,385]}$ Women with more severe DR were more likely to develop obstetric complications ${ }^{[385,386]}$ and those with proliferative changes accounted for higher incidence of congenital malformations and/or foetal death. ${ }^{[385]}$

- As pregnancy can induce progression of DR, the panel recommended pre-conception counselling for women, clearly explaining about the risk of progression of DR during pregnancy especially if they already have proliferative retinopathy. They should be advised on maintaining good glycaemic control before and throughout pregnancy under the guidance of a healthcare professional. In addition, the panel emphasized on the need for close follow-up during pregnancy and up to 1 year post-partum and monitoring for progression of DR and co-existing hypertension and renal disease, if any.

\section{Guarded prognosis after intra-ocular lens surgery}

- Though surgical interventions are crucial for cataract management, in most of the patients, particularly those with complicated cataracts, vision may not be restored. These patients eventually develop corneal decompensation, glaucoma and optic atrophy. ${ }^{[387]}$ Because the prognosis of retina is poor especially in the presence of mature cataract, the panel suggested that it is important to educate the patient about guarded prognosis for regaining vision after IOL surgery.

\section{EVIDENCE}

Though evidence from past studies suggests that prevalence of DR is low in Indians compared to other ethnic groups, emerging data indicate significant increase in prevalence of retinopathy in South Asians compared to Caucasians. ${ }^{[388]}$ Data from a populationbased study (CURES) indicate that the overall prevalence of DR in urban south Indian population was $17.6 \%$, with higher prevalence among men than in women $(21.3 \%$ vs. $14.6 \%$; $\mathrm{p}<0.0001)$ and among subjects with proteinuria $(\mathrm{p}=0.002) .{ }^{[389]}$ Similarly, prevalence of DR in western India was found to be $33.9 \%{ }^{[390]}$ Data from a recent population-based cross-sectional study suggests that one of 10 individuals in rural South India, above the age of 40 years, had evidence of DR. ${ }^{[391]}$ A metaanalysis of seven studies from India found $14.9 \%$ of known diabetes patients aged $\geq 30$ years and $18.1 \%$ among those aged $\geq 50$ years had DR. Furthermore, no linear trend was observed between age and the proportion with DR ${ }^{\left[{ }^{[392]}\right.}$ Duration of diabetes, $\mathrm{HbA1c}$, male gender, macro-albuminuria and insulin therapy 
were found to be strongly associated with increased risk of DR among South Indians. ${ }^{[393,394]}$ Moreover, the risk of nephropathy (OR: 5.3, p<0.0001) and neuropathy (OR: 2.9, p<0.0001) was significantly higher among T2DM patients with DR compared to those without DR ${ }^{[395]}$ After adjusting for age, gender, HbAlc, SBP, serum triglycerides, and duration of diabetes, DR was significantly associated with nephropathy $(\mathrm{p}=0.005)$ than with neuropathy. ${ }^{[395]}$ Another study showed HbA1c, BMI, duration of diabetes, microalbuminuria and peripheral neuropathy are contributing factors in the degree of retinopathy $(\mathrm{p}=0.001)$ and this correlation was explained by common mechanisms involved in tissue damage by all these factors. Microalbuminuria was positively correlated with the presence of retinopathy in T2DM patients and may be a marker for the risk of severe and proliferative retinopathy development. Microalbuminuria was associated cross-sectionally with the presence of retinopathy in patients with T2DM. This study suggests that microalbuminuria may be a marker for the risk of proliferative retinopathy development. ${ }^{[396]}$

\section{IMPLEMENTATION}

Sufficient number of trained general ophthalmologists and general physicians are required to develop an integrated DR model that facilitates early detection and create awareness on DR. Medical camps should be conducted for screening of diabetes and retinopathy, which will help to identify people at risk of sight-threatening DR and initiate treatment including laser photocoagulation or vitreous surgery. Mobile vans with a fundus camera or other low cost tools that can be used in remote rural areas should also be explored. However, successful implementation of program requires team approach, involving both administrative and voluntary organizations.

Telemedicine based DR screening costs less ( $\$ 10$ vs $\$ 25)$ than conventional retinal examination and the telemedicine-based digital retinal imaging examination has the potential to provide an alternative method with greater convenience and access for the remote and indigent populations. This cost effective technology driven model would prevent the screening costs and also help in the early detection of DR and prevent a common cause of blindness. Telemedicine should be encouraged to improve access, increase compliance with annual evaluation, at a low cost for patients with diabetes. ${ }^{[397]}$ Tele-diabetes shares some of the same attributes of tele-monitoring for other chronic conditions, such as congestive heart failure, stroke, and chronic obstructive pulmonary disease. In a pilot study conducted in Hungary on patients with diabetes, $30 \%$ of the patients had never participated in any ophthalmological screening, while $25.7 \%$ had DR of some grade based upon a standard fundus camera examination and UKbased DR grading protocol (Spectra ${ }^{\mathrm{TM}}$ software). Large majority of the patients were satisfied with the screening and found it reliable and acceptable to undertake examination under pupil dilation; $67.3 \%$ were willing to undergo nonmydriatic fundus camera examination again. Participants found digital retinal screening to be reliable and satisfactory. Telemedicine can be a strong tool, supporting eye care professionals and allowing for faster and more comfortable DR screening. ${ }^{[398]}$

\section{Neuropathy}

\section{RECOMMENDATIONS}

\section{Recommended Care}

- All patients with T2DM should be assessed for diabetic neuropathy at the time of initial diagnosis and annually

- Diagnose sensorimotor nerve damage by history and examination (10 g monofilament with or without temperature, non-traumatic pin-prick, vibration [128 Hz tuning fork], ankle reflexes), and/or simple quantitative testing (e. g. biothesiometer vibration perception). Use serum B12, thyroid function tests, creatinine/urea, and alcohol abuse and medication history to exclude other causes.

- Diabetic Neuropathy Symptom Score (NSS) and Neuropathy Disability Score (NDS) in T2DM population has been found to be a useful resource in evaluating diabetic sensorimotor polyneuropathy as an important bed side tool.

- Diagnose symptomatic (painful) diabetic neuropathy by excluding other possible causes of the symptoms. Manage by stabilizing blood glucose control, and treatment with tricyclic antidepressants, if simple analgesia is not successful. If a one month trial of tricyclic therapy is not successful, further treatment options include pregabalin/gabapentin and duloxetine, then tramadol and oxycodone.

- Weight gain and lifestyle measures need a reinforcement with the use of antidepressants and gabapentin and pregabalin.

- Further management normally requires referral to a pain control team. Be aware of the psychological impact of continuing symptoms, particularly if sleep is disturbed. In patients with diabetic neuropathy and co-morbid depression, anxiety and sleep loss, duloxetine should be preferred.

- A visual record of simple graphic tool to measure response to therapy must be mandated, which will save patients from over/unnecessary treatment.

- Tools e. g. pain scale should be encouraged in clinical practice.

- Diagnose erectile dysfunction by history (including medication history), exclusion of endocrine conditions (measure prolactin and testosterone), and a trial of a phosphodiesterase type-5 (PDE5) inhibitor (where not contraindicated by nitrate therapy). Consider other approaches such as intra-urethral or intracavernosal drugs and sexual and relationship counselling, where PDE5 inhibitors fail or cannot be used.

- Discourage use of alternative medicines as they can cause further complications.

- Diagnose gastroparesis by history, trial of a prokinetic drug (metoclopramide, domperidone) and if troublesome, by gastric emptying studies.

- Diagnose CV autonomic neuropathy by resting heart rate and heart rate response to provocation tests (lying-standing, Valsalva, deep breathing), and by lying and standing BP. Inform anaesthetists when relevant, where this is present.

- Every patient must undergo a simple assessment e. g. questionnaire-based assessment for depression. 


Limited Care
- Screen and diagnose sensorimotor nerve damage by history of symptoms, and sensory assessment by $10 \mathrm{~g}$ monofilament or tuning fork with/without
non-traumatic disposable pin-prick.
- NSS and NDS in T2DM population has been found to be a useful resource in evaluating diabetic sensorimotor polyneuropathy as an important bed side
tool.
- Manage symptomatic (painful) diabetic neuropathy by excluding other causes, stabilizing glycaemic control, and treatment with tricyclic antidepressants
if simple analgesia is not successful. Opiate analgesia may be necessary as locally available.
- Assess erectile dysfunction by history and examination and consider possible contributions of other medication or disease.

\section{BACKGROUND}

Neuropathy is among the most common life-threatening complication of diabetes that involves both peripheral and autonomic nerves, affecting up to half of all diabetic patients. Hyperglycaemia-induced polyol pathway, injury from AGEs, and enhanced oxidative stress have been implicated in its pathogenesis. ${ }^{[399,400]}$ Peripheral neuropathy in diabetes appears in several forms depending on the site, manifesting as sensory, focal/multifocal, and autonomic neuropathies. Diabetic neuropathy has resulted in more than $80 \%$ amputations after foot ulceration or injury. It is among the most common, expensive and disabling complications of diabetes, affecting approximately $30 \%$ of hospitalized patients with diabetes and $25 \%$ of patients with diabetes in the community. ${ }^{[401]}$ Approximately $30 \%$ patients either with known or newly diagnosed diabetes are suffering from diabetic neuropathy. ${ }^{[402,403]}$ In the cross-sectional survey of Indian patients with T2DM in CINDI and CINDI2 studies, diabetic neuropathy was prevalent in $13.15 \%$ and $13.2 \%$ patients, respectively. ${ }^{[373,374]}$ As per the Toronto Consensus Panel, diabetic polyneuropathy is a "symmetrical, length-dependent sensorimotor polyneuropathy attributable to metabolic and micro-vessel alterations as a result of chronic hyperglycaemia exposure and cardiovascular risk covariates". ${ }^{[404]}$ The most common form of diabetic neuropathy is the distal symmetrical polyneuropathy that involves both tibial and sural nerves. ${ }^{[405]}$ Presence of neuropathy is associated with significant morbidity, including recurrent foot infection and ulcers; impotence in men with diabetes, and sudden death in individuals with $\mathrm{CV}$ autonomic neuropathy. Neuropathic pain in patients with diabetes is commonly encountered in clinical practice. ${ }^{[405,406]}$ The present recommendations provide insights on the management aspects of diabetic neuropathy while exploring newer therapeutic options that have emerged in recent years.

\section{Rationale and Evidence}

\section{Detection of sensorimotor polyneuropathy}

- Though nerve conduction studies are powerful tools for identifying cases of diabetic neuropathy, ${ }^{[407]} \mathrm{NSS}^{[408]}$ and NDS ${ }^{[409]}$ in T2DM patients was found to be a useful resource for evaluating diabetic sensorimotor polyneuropathy as a bed side tool. ${ }^{[410,411]} \mathrm{A}$ cross sectional study in T2DM patients that examined the nerve conduction velocities of motor and sensory nerves, using NSS and NDS in patients of clinically detectable neuropathy showed significant electrophysiological changes with duration of T2DM.$^{[411]}$ Similar results were observed in another study where NSS and NDS together helped in prompt evaluation of diabetic sensorimotor polyneuropathy and also in diagnosing subclinical cases. ${ }^{[412-414]}$ A study that validated the use of NSS and NDS for clinical diagnosis of peripheral neuropathy in middle aged 855 T2DM patients showed that NSS and NDS can detect diabetic neuropathy with a sensitivity of $71.1 \%$ and specificity of $90 \%$ and was found to be simple, acceptable, reproducible and validated method for early diagnosis of diabetic neuropathy. ${ }^{[294,410]}$

- The panel emphasized on neurological examination using NSS and NDS as it is important bed side tool and a useful resource in evaluating diabetic sensorimotor polyneuropathy.

\section{Management of diabetic neuropathy [Figure 8]}

Diabetic peripheral neuropathic pain can be managed with several classes of drugs including tricyclic antidepressants, anticonvulsants, serotonin-norepinephrine reuptake inhibitors, opiates and opiate-like substances, and topical medications.

- Tricyclic antidepressants are recommended as first-line therapy for diabetic peripheral neuropathic pain in appropriate patients.

- Gabapentinis an anticonvulsant that is structurally related to $\gamma$-aminobutyric acid (GABA), a neurotransmitter that plays a role in pain transmission and modulation. In patients with a history of pain attributed to diabetic neuropathy gabapentin monotherapy was efficacious for the treatment of pain and sleep interference associated with diabetic peripheral neuropathy along with positive effects on mood and quality of life. ${ }^{[415]}$

- Duloxetine and pregabalin were approved by the USFDA in 2004 and tapentadol extended release was approved in 2012 for the treatment of painful diabetic neuropathy $(\mathrm{PDN}) \cdot{ }^{[416]}$

- Pregabalin is a potent gabapentinoid used in the management of PDN. Several double-blind placebocontrolled trials have reported the dose dependent (600 $\mathrm{mg} /$ daily) efficacy of pregabalin; however a number of side effects including mood disturbance, ankle oedema and sedation also have been reported. ${ }^{[417-419]}$

- Both duloxetine and pregabalin are effective; however, a significant improvement in QoL of patients was obtained by duloxetine with comparatively mild increase in the price. ${ }^{[420]}$ 


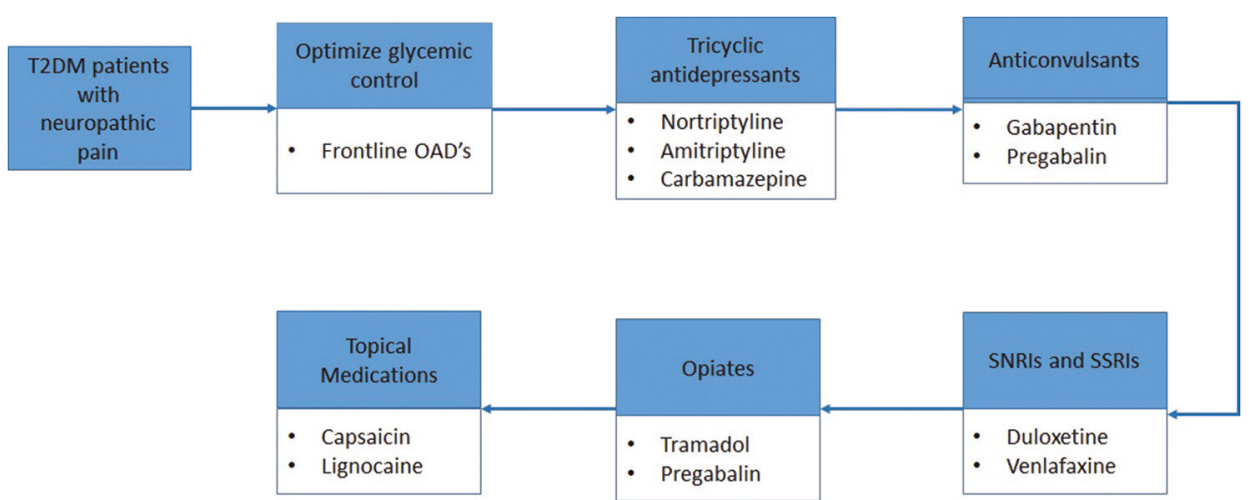

Figure 8: Management algorithm for neuropathy. OADs: Oral antidiabetics; SNRIs: Serotonin-norepinephrine reuptake inhibitors; SSRIs: Selective serotonin reuptake inhibitors

- Duloxetine is a selective inhibitor of reuptake of both 5-hydroxytryptamine and norepinephrine. ${ }^{[421,422]}$ Results from randomized-controlled clinical trials reveal that duloxetine provides significantly more diabetic neuropathic pain relief than either placebo or routine care with higher degree of safety and tolerability. ${ }^{[423-425]}$ Moreover, a recent Cochrane collaboration review including data from eight studies and 2728 participants report that both $60 \mathrm{mg}$ and $120 \mathrm{mg}$ daily doses of duloxetine were efficacious, but lower doses were not associated with improvement in the PDN management. ${ }^{[226]}$

- Tapentadol, an opioid analgesic, may act via opioid spinalsupraspinal synergy, as well as intrinsic spinally mediated $\mu$-opioid receptor agonist-norepinephrine reuptake inhibitor effect. ${ }^{[427]}$ The efficacy and safety of tapentadol was also published in several clinical trials. ${ }^{[428,429]}$ Tramadol may also be used for pain management however, there is only modest information about the use of tramadol in neuropathic pain, primarily from small, largely inadequate studies ${ }^{[430]}$ Further, a fixed-dose combination of tramadol/paracetamol might be a useful pharmacological option for chronic pain management, particularly in elderly patients..$^{[431]}$

- Neuropathic pain can be severe and can impact quality of life, limit mobility, and contribute to depression and social dysfunction. ${ }^{[432]}$ Management of underlying depression is a must to improve QoL. Tricyclic antidepressants, venlafaxine, carbamazepine, and topical capsaicin, although not approved for the treatment of painful diabetic neuropathy, may be effective and considered for the treatment. ${ }^{[433-436]}$

- Capsaicin has been used with some success in the treatment of patients with PDN. It binds to the receptor that opens the TRPV1 causing sodium and calcium influx and substance P release occurs, Repeated TRPV1 exposure to capsaicin causes substance $\mathrm{P}$ depletion and TRPV1 desensitization and defunctionalization. ${ }^{[437]}$

- Lidocaine blocks the voltage-gated sodium channels and stabilizes the neuronal membrane potential, resulting in a reduction of ectopic discharges and raised peripheral ectopic discharge threshold causing reduced pain transduction. ${ }^{[438]}$

\section{IMPLEMENTATION}

Appropriate protocols should be developed for sensory testing and may include formal assessment using the NSS and NDS. Recommended medications should be available according to the level of resources. Medical teams need to remain trained in the diverse manifestations of autonomic neuropathy.

\section{Diabetic Kidney Disease}

\section{RECOMMENDATIONS}

\section{Recommended Care}

- Kidney function should be assessed at diagnosis and annually by:

- Urine test for albuminuria

- Measurement of serum creatinine and calculation of eGFR

- Urinary albumin to creatinine ratio (ACR) measurement in an early morning first void (mid-stream) spot specimen is the preferred method for assessment of microalbuminuria/proteinuria. Where a first void specimen is not possible or practical, a random spot urine specimen is acceptable. ACR can be measured in the laboratory or at site-of-care.

- Control hyperglycaemia, exclude urinary or systemic infections, or pyrexia and avoid strenuous exercise before testing for albuminuria.

- If ACR is raised (microalbuminuria) i.e. ACR $>30 \mathrm{mg} / \mathrm{g}$ creatinine, repeat ACR twice over the following four months: 
- Microalbuminuria is confirmed if ACR is elevated in two out of three tests, in the absence of infection or overt proteinuria

- If both repeat tests are not raised, check again annually

- An ACR $>300 \mathrm{mg} / \mathrm{g}$ indicates macroalbuminuria

- DKD is diagnosed on the basis of a raised urine albumin/protein or a reduced eGFR $\left(<60 \mathrm{~mL} / \mathrm{min} / 1.73 \mathrm{~m}^{2}\right)$ calculated from the Chronic Kidney Disease Epidemiology Collaboration (CKD-EPI) equation. CKD-EPI is the preferred formula.

- The Modification of Diet in Renal Disease (MDRD) formula for calculation of eGFR is not validated above 70 years age and in Indian patients.

- For patients $<18$ years of age (including infants, toddlers, children, and teens), the Bedside Schwartz equation should be used.

- Individuals with DKD should be managed as follows:

- Identified high-risk individuals (hypertensives, duration of diabetes $>3-5$ years, family history of nephropathy/HF/ASCVD) must get preference for SGLT2 inhibitors for glycemic management if feasible and accepted by patients (eGFR $>30 \mathrm{~mL} / \mathrm{min} / 1.73 \mathrm{~m}^{2}$ )

- Use angiotensin converting enzyme (ACE)-inhibitors or angiotensin receptor blockers (ARBs) in individuals with micro-or macro-albuminuria, titrated to maximum tolerated dose

- Intensify management of BP (target $\leq 130 / 80 \mathrm{~mm} \mathrm{Hg}$ ) using BP lowering medications and dietary modification (low salt and reduced protein intake)

- Intensify management of blood glucose

- Monitor ACR, eGFR and serum potassium

- Advise limiting protein intake to $1 \mathrm{~g} / \mathrm{kg}$ of high biological value protein daily. In those with advancing CKD, restrict to $0.8 \mathrm{~g} / \mathrm{kg}$ daily with advice for caution in patients consuming non-vegetarian diet

- Intensify other renal and CV protection measures

- Assessment and management of anaemia and bone disease and appropriate vaccination

- Smoking leads to progression to end-stage renal disease (ESRD) in diabetes so patients must be counselled for quitting smoking

- Consider referral to nephrologists when there is uncertainty about the aetiology of kidney disease, difficult management issues (stress, obesity, high uric acid, UTIs, anaemia for timely use of Erythropoietin analogues, BP to targets, Nocturnal BP control stressed)

- Agree to a referral criteria for specialist renal care between local diabetes specialists and nephrologists. Referral criteria might include eGFR $<30 \mathrm{~mL} / \mathrm{min} / 1.73 \mathrm{~m}^{2}$, progressive deterioration of kidney function, persistent proteinuria, biochemical or fluid retention problems or difficult diagnosis (to rule out non diabetic renal disease where fundus is normal and no proteinuria).

- Rule out non-diabetic kidney disease in patients with early onset of nephropathy ( $<5$ years), absence of retinopathy, heavy proteinuria, presence of active urinary sediments or unexplained rapid decline in eGFR.

\section{Limited Care}

- Check annually for proteinuria in an early morning urine sample (or a random sample) using a dipstick. If test is positive exclude UTIs by microscopy (and culture if possible).

- Measure serum creatinine and calculate eGFR annually.

- A simple inexpensive screening procedure for urinary protein excretion which can be used as a diagnostic test in outpatient has been reported in Indian population. Estimated proteinuria is useful in serial evaluation of kidney function.

- Manage those with proteinuria as follows:

- Consider use of ACE inhibitors or ARBs and SGLT2 inhibitors unless contraindicated or issues with tolerability

- Aim for BP $\leq 130 / 80 \mathrm{~mm} \mathrm{Hg}$ using any BP lowering medication and control of salt intake [Table 13]

- Aim to achieve targets for blood glucose control

- Aim to improve lipid profile using available medications

- Check proteinuria status annually

- Measure serum creatinine and calculate eGFR annually

\section{BACKGROUND}

Previously known as diabetic nephropathy, DKD is defined as diabetes with albuminuria (ratio of urine albumin-tocreatinine $\geq 30 \mathrm{mg} / \mathrm{g}$ ), impaired glomerular filtration rate $\left(<60 \mathrm{~mL} / \mathrm{min} / 1.73 \mathrm{~m}^{2}\right)$, or both and is now recognised as the strongest predictor of mortality in patients with diabetes. ${ }^{[439]}$ It is a leading cause of ESRD affecting 20-30\% diabetes patients, and is associated with increased CV mortality. ${ }^{[40]}$ It affects $10-40 \%$ of T2DM patients who eventually suffer from kidney failure. ${ }^{[441,442]}$ In the cross-sectional survey of Indian patients with T2DM in CINDI and CINDI2 studies diabetic nephropathy was prevalent in $1.06 \%$ and $0.9 \%$ patients respectively. ${ }^{[373,374]}$ Cost of treatment for advanced CKD is substantial. Less than $10 \%$ of ESRD patients have access to any kind of renal replacement therapy. ${ }^{[43,444]}$ Thus, in a country with limited resources, it becomes appropriate to direct efforts toward prevention of DKD rather than the treatment.

\section{Pathophysiology of diabetic kidney disease}

The pathophysiological mechanisms of DKD are complex and are often evident by intrarenal hypertension, compromised GFR and microalbuminuria. Microalbuminuria, is the first and most critical manifestation of diabetic nephropathy, which when progressed to overt albuminuria (increased albumin levels in the urine) indicates severe renal dysfunction culminating to renal failure ${ }^{[445]}$ The presence of microalbuminuria is a powerful marker of cardiovascular disease and all-cause mortality. ${ }^{[446]}$ Thus, the presence of diabetes, particularly accompanied by microalbuminuria, is most often considered a warning signal for $\mathrm{CV}$ risks in patients with diabetes. 


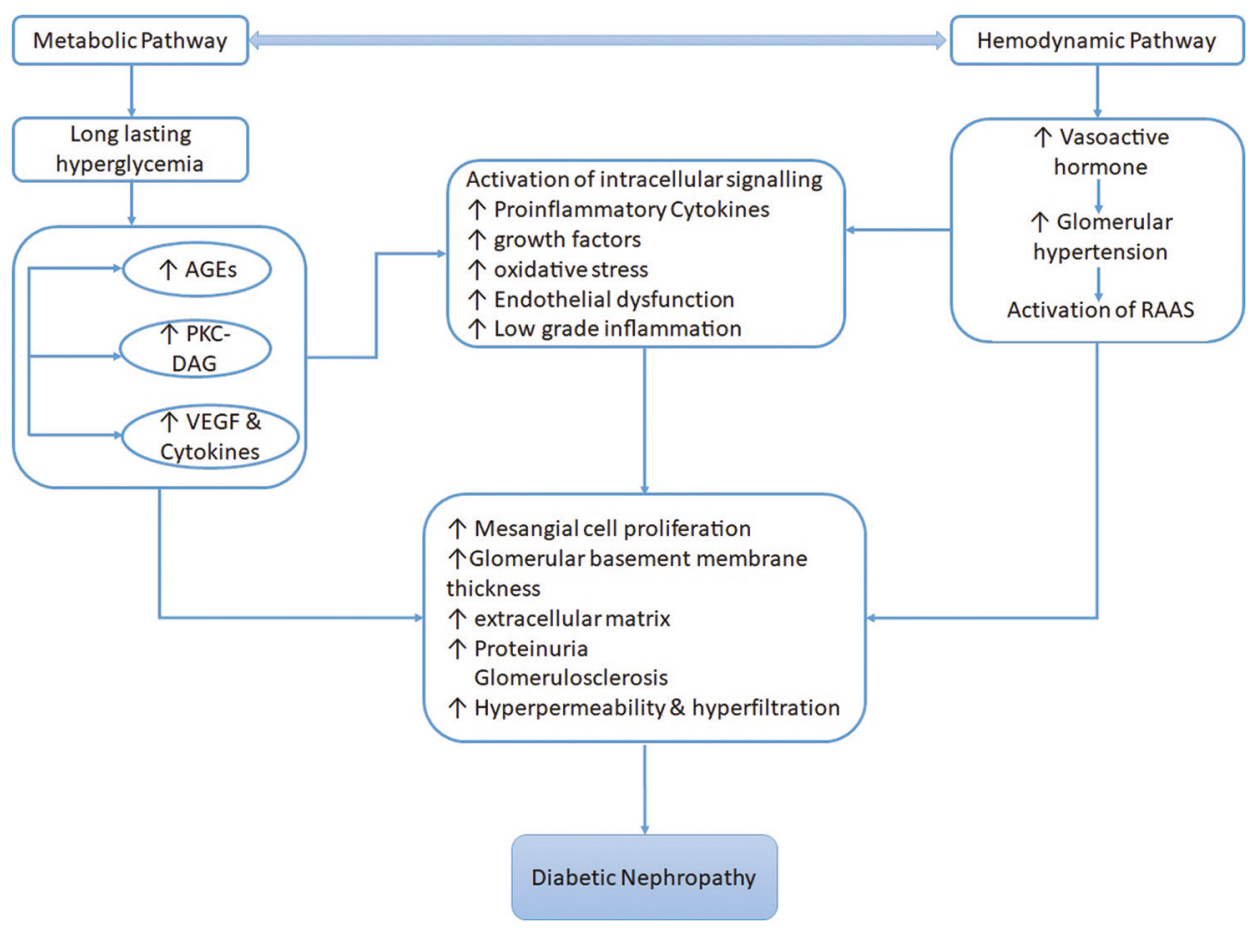

Figure 9: Pathophysiology pathways in diabetic kidney disease. AGEs: Advanced glycation end products; PKC-DAG: Protein kinase C-diacylglycerol; RAAS: Renin-angiotensin-aldosterone-system; VEGF: Vascular endothelial growth factor

DKD is characterized by a constellation of histopathological changes beginning from glomerular hyper filtration causing glomerular basement membrane thickening, progressive accumulation of extracellular matrix in glomerular mesangium and tubulointerstitium, causing mesangial expansion and Kimmelstiel-Wilson nodules (an aggregation of mesangial cells and mesangial matrix), arterial hyalinosis, and tubulointerstitial changes [Figure 9]. Additionally, podocyte dropout is also a critical factor for DKD development. Podocytes are known to distort and change their size and shape to accommodate or cover the openings created by the basement membrane thickening causing them to shift or dropout.

In India, with increase in the prevalence of diabetes, it becomes imperative to evolve definite guidelines for detection of diabetic nephropathy and suggest practical clinical recommendations to combat it.

Improving glycaemic control, aggressive antihypertensive treatment, and the use of ACE inhibitors or ARBs will slow down the rate of progression of nephropathy. ${ }^{[47,448]}$ In addition, protein restriction and other treatment modalities such as phosphate lowering may have benefits in selected patients. ${ }^{[449]} \mathrm{A}$ due consideration should be given to normoalbuminuric kidney disease in patients with T2DM. Although the serum creatinine is usually normal, most of the normoalbuminuric patients with DKD according to fact have an eGFR $<60 \mathrm{~mL} / \mathrm{min} / 1.73 \mathrm{~m}^{2}$ per the MDRD formula. However, as expected, because of normoalbuminuria and other favorable characteristics, their risk for DKD progression or death is lower. ${ }^{[450]}$

\section{Rationale and Evidence} Identification and monitoring and diabetic kidney disease Persistent microalbuminuria is the earliest sign of diabetic nephropathy or DKD. The diagnostic reference standard for defining microalbuminuria is detection of 30 to 300 $\mathrm{mg}$ of albumin in a 24-h urine sample and is the first-line annual screening test for most persons with diabetes. It is recommended that once a screening test detects microalbuminuria, it should be confirmed with additional spot urine tests over the next three to six months. The Kidney Disease Outcomes Quality Initiative (KDOQI)-Clinical Practice Guidelines and Clinical Practice Recommendations for Diabetes and Chronic Kidney Disease by the National Kidney Foundation (NKF), recommend that patients with diabetes should be screened annually for DKD: 5 years after the diagnosis of type 1 diabetes and from onset/diagnosis of type 2 diabetes. The presence of albuminuria must be evaluated based on the UAE concentration or Urinary albumin-tocreatinine ratio (UACR) in untimed (spot) urine specimens and by estimating the glomerular filtration rate from serum creatinine measurements by using prediction equations. ${ }^{[453,454]}$ The ADA recommends identifying and monitoring DKD based upon assessments of kidney function with an estimated GFR (eGFR), $60 \mathrm{~L} / \mathrm{min} / 1.73 \mathrm{~m}^{2}$, or kidney damage by estimation of albuminuria $30 \mathrm{mg} / \mathrm{g}$ creatinine along with annual screening for microalbuminuria. Clinical recommendation by the ADA for DKD screening also suggest that persons with type 1 or 2 diabetes and microalbuminuria should continue to be tested 
for albuminuria annually to monitor disease progression and response to therapy. ${ }^{[455]}$

- EPE is a method of estimating ACR in a random urine sample to assess renal function in patients with diabetes. EPE was found to be useful in serial evaluation of kidney function in Indian patients with diabetes. ${ }^{[45,457]}$ Moreover, EPE is a simple and inexpensive screening procedure for urinary protein excretion, which can be used as a diagnostic test in outpatient wards, particularly in developing countries like India.

- As EPE is an inexpensive screening procedure to assess kidney function, the panel recommended it for use in Indian population who are at risk of diabetic nephropathy.

- Screening of microalbuminuria and estimation of glycated albumin can help in the clinical management of diabetic nephropathy. ${ }^{[458]}$ Screening for albuminuria by measuring urine albumin concentration or estimating ACR is acceptable in Asian population. ${ }^{[459]}$ However, evidence suggests that vigorous exercise even for short periods (15-20 min) leads to ACR above the microalbuminuria threshold even in healthy participants. ${ }^{[460,461]}$

- On the basis of evidence, the panel suggested that physicians should ask about recent vigorous exercise and avoid measuring urine albumin excretion for at least $24 \mathrm{~h}$ in the presence of same.

- Microalbuminuria shows a strong association with increased CVD risk in diabetic patients in Indian population. ${ }^{[462-464]}$

\section{Management of hyperglycaemia in patients with diabetic kidney disease}

In patients with $\mathrm{DKD}$, when selecting and dosing glucoselowering drugs, renal function has to be assessed and periodically monitored during treatment to detect changes that may affect drug metabolism and excretion. While mild renal insufficiency can be treated with most OADs, patients with DKD stage 3-5, most often require treatment adjustments according to the degree of renal insufficiency.

Combinations of therapies are available for the management of hyperglycaemia in patients with type 2 diabetes. Metformin is a first-line agent in all patients, including patients with DKD. Second generation sulphonylureas are also commonly used. Although, reduction in $\mathrm{HbAlc}$ is modest with an average between 0.5-1.0 $\%$, DPP-4 inhibitors can be safely used at the appropriate dose in DKD. SGLT2 inhibitors and DPP-4 inhibitors are responsible choices in moderate to severe cases of DKD [Table 14].

\section{Oral antidiabetics that exert renoprotection}

- Evidence suggests that two oral hyperglycaemic agents DPP-4 inhibitors ${ }^{[465]}$ and SGLT2 inhibitors, exert renoprotective effects in patients with diabetes. SGLT2 inhibitors are indicated to improve glycaemic control in adults with T2DM by reducing the reabsorption of filtered glucose. They can also lower the renal threshold for glucose, thereby increasing urinary glucose excretion.

- While these medications have been used safely in patients with Stage 3 CKD (eGFR down $<30 \mathrm{~mL} / \mathrm{min}$ ), the glycaemic reduction response to the SGLT2 inhibitors declines with decreasing kidney function, as a decrease in eGFR results in a decrease in urinary glucose excretion.

- Canagliflozin has been approved for use in patients with eGFR $>45 \mathrm{~mL} / \mathrm{min} / 1.73 \mathrm{~m}^{2}$, with dose limited to $100 \mathrm{mg}$ once daily in patients with eGFR $45 \leq 60 \mathrm{~mL} / \mathrm{min} / 1.73 \mathrm{~m}^{2}$. Empagliflozin can also be used in patients with an eGFR down to $45 \mathrm{~mL} / \mathrm{min} / 1.73 \mathrm{~m}^{2}$, while dapagliflozin is approved in patients with an eGFR down $\geq 60 \mathrm{~mL} / \mathrm{min} / 1.73 \mathrm{~m}^{2}$. Regular assessment of renal function is recommended with use of any of these SGLT2 inhibitors.

- Recently completed CREDENCE study reported that at a median follow-up of 2.62 years, the risk of kidney failure and cardiovascular events was lower in the canagliflozin group than in the placebo group. ${ }^{[466]}$ In the Canagliflozin Cardiovascular Assessment Study (CANVAS) Program study, canagliflozin treatment was associated with a reduced risk of sustained loss of kidney function, attenuated eGFR decline, and a reduction in albuminuria. These encouraging results suggest that canagliflozing exerts renoprotective effect in patients with T2DM. ${ }^{[467]}$

- The EMPA-REG OUTCOME trial evaluated the non-inferior cardiovascular safety of empagliflozin in highCV-risk T2D patients with an estimated glomerular filtration rate (eGFR) of at least $30 \mathrm{~mL} / \mathrm{min} / 1.73 \mathrm{~m}^{2}$. Empagliflozin reduced the rate of new onset or worsening nephropathy, which were defined as new-onset microalbuminuria, doubling of creatinine, and eGFR $\leq 45 \mathrm{~mL} / \mathrm{min} / 1.73 \mathrm{~m}^{2}$, initiation of renal replacement therapy, and death due to renal disease (hazard ratio [HR]: $0.61,95 \%$ confidence interval [CI]: 0.53-0.70; $\mathrm{p}<0.0001) .{ }^{[468]}$

- Results of the DECLARE-TIMI 58 cardiovascular outcomes trial suggest that, in patients with T2DM, Dapagliflozin prevented and reduce the progression of renal disease. ${ }^{[281]}$

\section{Protein restriction}

- IDF recommends limiting protein intake to $1 \mathrm{~g} / \mathrm{kg}$ body weight daily among individuals with $\mathrm{DKD}$, if they are found proteinuric. Similarly, ADA recommends protein intake should be $0.8 \mathrm{~g} / \mathrm{kg} /$ body weight $/$ day in patients with DKD. ${ }^{[451]}$ In the Indian context, the source of protein is mainly from vegetable and animal oils and daily protein consumption is about $0.6-0.8 \mathrm{~g} / \mathrm{kg}$ body weight. ${ }^{[469]}$ Furthermore, protein content in non-vegetarian diet was found to be higher when compared to the vegetarian diet. ${ }^{[470]}$ In addition, evidence suggests that animal protein may aggravate the risk of diabetes. ${ }^{[471]}$ Therefore the panel emphasized on protein restriction and avoiding extra protein intake, particularly in non-vegetarians with nephropathy.

\section{Smoking}

- Smoking is associated with hyperglycaemia, dyslipidemia and decline in GFR which leads to the progression of ESRD in patients with diabetes. ${ }^{[472,473]}$ Smoking tends to induce albuminuria and abnormal renal function through formation of advanced glycated end products (AGEs), which are responsible for advanced vascular permeability and 
kidney damage. ${ }^{[474]}$ A recent systematic review reported that consumption of $\geq 15$ packs of cigarettes/year increases the risk of progression of DKD ${ }^{[475]}$ Moreover, data from a recent study in India suggests that compared to non-smokers the prevalence of microalbuminuria in smokers was 4-fold higher. ${ }^{[476]}$

- The panel opined that patients must be counselled against tobacco use and encouraged to quit smoking to reduce the risk of progression to ESRD.

\section{Referral to specialist}

- The panel endorsed IDF recommendation on referral criteria; however, it was suggested that, most of the

\begin{tabular}{|c|c|}
\hline Guideline & Recommendation \\
\hline \multirow[t]{3}{*}{$\begin{array}{l}\text { ADA } \\
2019^{[451]}\end{array}$} & $\begin{array}{l}<140 / 90 \mathrm{mmHg} \text { is recommended to decrease CVD } \\
\text { mortality and slow down DKD progression }\end{array}$ \\
\hline & $\begin{array}{l}\text { Lesser targets such as }<130 / 80 \mathrm{mmHg} \text { might be } \\
\text { considered in individuals with albuminuria and at } \\
\text { increased risk of CVD and CKD progression }\end{array}$ \\
\hline & $\begin{array}{l}\text { While achieving }<130 \mathrm{mmHg} \text { SBP target, especially in } \\
\text { old people, care should be taken to avoid DBP levels } \\
<60-70 \mathrm{mmHg}\end{array}$ \\
\hline \multirow[t]{2}{*}{$\begin{array}{l}\text { KDIGO } \\
2012^{[452]}\end{array}$} & $\begin{array}{l}\text { In DKD patients, not requiring dialysis, with } \mathrm{UAE}<30 \\
\mathrm{mg} / \text { day and office } \mathrm{BP} \text { consistently below } 140 / 90 \mathrm{~mm} \mathrm{Hg} \text {, } \\
\text { a target of } \leq 140 / 90 \mathrm{mmHg} \text { is recommended }\end{array}$ \\
\hline & $\begin{array}{l}\text { In DKD patients, not requiring dialysis, with } \mathrm{UAE}>30 \\
\mathrm{mg} / \text { day and office } \mathrm{BP} \text { consistently }>130 / 80 \mathrm{~mm} \mathrm{Hg} \text {, a } \\
\text { target of } \leq 130 / 80 \mathrm{mmHg} \text { is recommended }\end{array}$ \\
\hline \multicolumn{2}{|c|}{$\begin{array}{l}\text { ADA: American Diabetes Association, BP: Blood pressure, CKD: Chronic } \\
\text { kidney disease, CV: Cardiovascular, CVD: CV disease, DKD: Diabetic } \\
\text { kidney disease, SBP: Systolic BP, DBP: Diastolic BP, UAE: Urinary } \\
\text { albumin excretion }\end{array}$} \\
\hline
\end{tabular}

patients at this stage of diabetic nephropathy require a specialist care which may not be available at primary care or single physician centre. Hence, local diabetes specialists should refer the patient to specialist renal care centre/nephrologist. Likewise, nephrologists should refer patients to specialist renal care if the patient presents with following condition:

- $\quad$ eGFR $<30 \mathrm{~mL} / \mathrm{min} / 1.73 \mathrm{~m}^{2}$

- progressive deterioration of kidney function

- persistent proteinuria, biochemical or fluid retention problems or

- difficulty in diagnosis (to rule out non diabetic renal disease where fundus is normal and proteinuria is not present).

\section{Indian evidence}

- Prevalence of microalbuminuria is strongly associated with age, DBP, HbAlc, FPG and duration of diabetes. ${ }^{[477,478]}$

- A positive co-relation between urine albumin excretion rate and eGFR $<60 \mathrm{~mL} / \mathrm{min} / 1.73 \mathrm{~m}^{2}$ was observed indicating that these two parameters provide a complimentary benefit in management of CKD. ${ }^{[479]}$

- Vitamin D deficiency can have significant impact on albuminuria. Therefore supplementation with calcitriol should be considered in these patients as it has been shown to provide beneficial effects on microalbuminuria. ${ }^{[480]}$

\section{IMPLEMENTATION}

Management of DKD requires access to healthcare professional, laboratory for ACR and creatinine estimations, and availability of multiple blood-pressure-lowering medications in particular renin-angiotensin system blockers.

\section{Table 14: Dose adjustment for oral antidiabetics agents for patients with diabetic kidney disease}

\begin{tabular}{|c|c|}
\hline Class & Dose adjustments \\
\hline \multirow[t]{4}{*}{ Metformin } & Metformin can be used till to GFR 30 \\
\hline & GFR $\geq 45-59$ : use caution with dose and follow renal function \\
\hline & GFR $\geq 30-44:$ max dose $1000 \mathrm{mg} /$ day or use $50 \%$ dose reduction. Follow renal function every 3 months \\
\hline & GFR: $<30$ : avoid use \\
\hline \multirow[t]{4}{*}{ Second-generation SU } & Glipizide: GFR <30: Use with caution \\
\hline & Glimepiride: GFR $<60$ : Use with caution; $<30$ : Avoid use \\
\hline & Glyburide: Avoid use \\
\hline & Gliclazide-upto GFR 30: No dose adjustment; $<30$ : Low dose preferred \\
\hline TZD & No dose adjustment \\
\hline \multirow{2}{*}{$\begin{array}{l}\text { Alpha-glucosidase } \\
\text { inhibitors }\end{array}$} & Acarbose: Serum creatinine $>2 \mathrm{mg} / \mathrm{dL}$ : Avoid use \\
\hline & Miglitol: GFR $<25$ or serum creatinine $>2 \mathrm{mg} / \mathrm{dL}$ : Avoid use \\
\hline \multirow[t]{6}{*}{ DPP-4 inhibitor } & Sitagliptin: GFR $\geq 50: 100 \mathrm{mg}$ daily; GFR 30-49: $50 \mathrm{mg}$ daily \\
\hline & GFR $<30: 25$ mg daily \\
\hline & Saxagliptin: GFR $>50: 2.5$ or $5 \mathrm{mg}$ daily; GFR $\leq 50: 2.5 \mathrm{mg}$ daily \\
\hline & Linagliptin: No dose adjustment \\
\hline & Alogliptin: GFR $>60: 25 \mathrm{mg}$ daily; GFR 30-59: $12.5 \mathrm{mg}$ daily; GFR $<30: 6.25 \mathrm{mg}$ daily \\
\hline & Tenaligliptin $20 \mathrm{mg} /$ day - No dose adjustment \\
\hline \multirow[t]{3}{*}{ SGLT2i } & Canagliflozin: GFR 45-<60: Maximum dose $100 \mathrm{mg}$ OD; GFR $<45$ : avoid use \\
\hline & Dapagliflozin: GFR <60: Avoid use \\
\hline & Empagliflozin: GFR <45: Avoid use \\
\hline
\end{tabular}

GFR: Glomerular filteration rate, SU: Sulfonylureas, OD: Once daily, TZD: Thiazolidinedione, DPP-4: Dipeptidyl peptidase, SGLT2i: Sodium-glucose co-transporter 2 inhibitors 


\section{Chronic Complications 2: Diabetic Foot and Peripheral Arterial Disease}

\section{Diabetic Foot}

\section{ReCOMmendations}

\section{Recommended Care}

- Assess feet of patients with diabetes at every visit for lesions requiring active treatment and for risk factors for ulcer and amputation:

- History of previous foot ulceration or amputation, symptoms of peripheral arterial disease (PAD), physical or visual difficulty in self-foot-care

- Foot deformity (hammer or clawed toes, bone prominences), visual evidence of neuropathy (dry skin, dilated veins) or incipient ischemia, callus, nail deformity or damage. Patient footwear should also be assessed

- Detection of neuropathy by $10 \mathrm{~g}$ Semmes Weinstein monofilament (or $128 \mathrm{~Hz}$ tuning fork); a biothesiometer (to assess vibration perception threshold) is an option for quantitative assessment (cut-off point for ulcer risk $>25$ volts) and non-traumatic pin-prick.

- Palpation of foot pulses (dorsalis pedis and posterior tibial). Doppler ultrasound examination or ankle: brachial pressure (ABI) ratio $(<0.9$ for occlusive vascular disease) may be used where pulses are diminished to quantify the abnormality.

- Discuss the reasons for foot review with each patient with diabetes, as part of the foot-care educational process.

- Must emphasize to completely refrain from walking bare foot, including at visits to religious places.

- Timely screening and early detection of diabetic neuropathy may help in prevention of the progression to diabetic foot.

- Agree upon a foot-care plan based on the findings of annual foot review with each person with diabetes. Assess and provide necessary foot-care education according to individual needs and risks of ulcer and amputation.

- Classify and manage according to risk classification level based on findings of foot assessment:

- People with foot ulceration or infection require the following management:

- Pressure off loading

- Refer to multidisciplinary foot-care team within $24 \mathrm{~h}$ for:

- Appropriate wound management, dressings and debridement as indicated

- Infections should be classified as mild (superficial with minimal cellulitis), moderate (deeper than skin or more extensive cellulitis), or severe (accompanied by systemic signs of sepsis). Consideration of systemic antibiotic therapy (often longer term) for extensive cellulitis or bone infection as indicated.

- First-line medications: generic penicillin, cephalosporins, macrolides, clindamycin and/or metronidazole, as indicated

- Second-line medications: amino-quinolones co-amoxicillin, imipenem.

- Probing to bone, radiology and scans, magnetic resonance imaging and biopsy where indicated for suspected osteomyelitis

- Reduce weight bearing, relief of pressure (walking with crutches, rest) off loading and optimal pressure distribution (casting, if indicated)

- Investigation and treatment (referral) for vascular insufficiency

- Specialist therapeutic footwear and orthotic care (e. g. insoles) and individualized discussion of prevention of recurrence, when ulcer has healed

- Optimal blood glucose control

Amputation should not be considered unless:

- A detailed vascular evaluation has been performed by the vascular team

- Ischemic rest pain cannot be managed by analgesia or revascularization

- A life-threatening foot infection cannot be treated by other measures

- A non-healing ulcer is accompanied by a higher burden of disease that would result in amputation.

\section{Risk classification level}

No added risk:

No risk factors; no previous history of foot ulcer or amputation At risk:

One risk factor; no previous history of foot ulcer or amputation

High risk:

$\geq 2$ risk factors; previous ulcer or amputation (very high risk)

\section{Management}

- Provide structured foot-care education and annual review.

- Foot-care team to regularly review every 6 months.

- At each review:

- Inspect both feet - ensure provision of local management as indicated

- Educate patient to wash feet daily (with careful drying, particularly between the toes), use emollients to lubricate dry skin, cut toe nails straight across, and avoid using chemical agents or plasters or any other technique to remove callus or corns

- Evaluate footwear - provide appropriate advice

- Enhance foot-care education

- Foot-care team to frequently review every 3-6 months.

- Educate patient to self-monitor foot skin temperatures once per day to identify any early signs of foot inflammation to prevent a first or recurrent plantar foot ulcer.

- At each review:

- Inspect both feet - ensure provision of local management as indicated

- Evaluate footwear - provide advice and specialist insoles and shoes if indicated

- Consider need for vascular assessment or referral, if indicated

- Evaluate and ensure appropriate provision of intensified foot-care education 


\section{Limited Care}

- Risk assessment and classification: Similar to 'recommended care' but with sensory assessment by $10 \mathrm{~g}$ monofilament or tuning fork, with or without non-traumatic disposable pin-prick only and peripheral circulation assessment by palpation of pedal pulses.

- NSS and NDS in T2DM population have been found to be a useful resource and an important bed-side tool in evaluating diabetic sensorimotor polyneuropathy.

- Classification of infection: Similar to 'recommended care' but antibiotic therapy would be with generic penicillin, quinolones, macrolides and/or metronidazole, given intravenously for deep tissue infections and adjusted by response or culture results.

- Vascular referral would be according to findings and local revascularization facilities.

\section{BACKGROUND}

Peripheral neuropathy, peripheral vascular disease (PVD), if occurs only in the arteries, it is called PAD, gait disorders, ischemia, foot ulcers, infections, gangrenes, Charcot neuroarthropathy and lower extremity amputations are some of the lower limb complications observed in patients with diabetes. ${ }^{[481,482]}$ Lack of sanitation and hygiene, socio-cultural practices such as barefoot walking in doors and at religious places, walking on fire, a lack of awareness on the use of proper footwear and a dearth of foot care clinics, together with economic factors exacerbate diabetic foot complications in India. ${ }^{[483,484]}$

\section{Diabetic foot ulcers and peripheral arterial disease}

In India, DFUs [Figure 10] affect $25 \%$ of total diabetic patients during their lifetime and is one of the most common reasons for hospitalization and amputation in patients. The cost of diabetic foot care in India is one of the highest in the world ${ }^{[485]}$ ( $\sim 5.7$ years of average annual income).

Neuropathy and PVD are important risk factors in diabetic foot infections ${ }^{[487-489]}$ that are a major cause of amputation and mortality amongst patients with diabetes in India. ${ }^{[490,491]}$ While the prevalence of neuropathy has been estimated to be $\sim 15 \%,{ }^{[488]}$ PVD prevalence varies across geographies. A lower prevalence has been reported among Indians compared to Western countries (13\% versus $48 \%){ }^{[489]}$ a younger patient population, shorter lifespan of patients with diabetes, along with a lower proportion of smokers could be plausible reasons for this difference. ${ }^{[487,489,492]}$ Presence of PVD and claudication in patients with diabetes is an indication of PAD, which leads to a higher risk of cardiovascular mortality and morbidity. ${ }^{[487]}$

Despite prevalence of PAD being estimated to be 50$60 \%$ amongst patients with DFU, appropriate and timely diagnosis of PAD [Figure 11] is still a major concern and a leading cause of amputation in patients with diabetes ${ }^{[493]}$ [Annexure 3].

\section{Charcot neuroarthropathy}

The Charcot neuroarthropathy is a major consequence of diabetic neuropathy that leads to bone deformities, subluxation and dislocation resulting in inflammation characterized by a reddish, hot, swollen foot: the Charcot foot. The classic "rocker-bottom" foot is an example of end-stage disease with severe fracture dislocation, collapse of the midfoot, dorsal dislocation of the metatarsals and plantar dislocation of the tarsal bones. ${ }^{[486,494,495]}$ The prevalence ranges from $0.4 \%-13 \%$ among patients with diabetes, with a mortality rate of $28 \%$. Using X-ray and MRI, the detection rates increase to $\sim 30 \%$ and $75 \%$, respectively. ${ }^{[494]}$

Diagnosis of Charcot foot is often delayed or missed and can lead to severe foot deformity, ulceration, infection and/or lower extremity amputation. ${ }^{[495]}$ Initial diagnosis includes testing for sensory neuropathy done using a 128$\mathrm{Hz}$ tuning fork or a $10 \mathrm{-g}$ monofilament or by testing lighttouch perception. Treatment is mostly conservative, with early treatment options being off-loading with total contact casting (TCC) and non-weight bearing in a cast or wheelchair until the acute inflammatory process subsides (may take weeks or months). A prefabricated orthosis device such as a Charcot Restraint Orthotic Walker (CROW) may also be used. Overall, the period of fixation depends on reduction of edema and a drop in skin temperature below $2^{\circ} \mathrm{C}$ compared to contralateral extremity. Late treatment requires reconstructive surgery to repair the deformity and obtain a plantar-grade foot. Off-loading using therapeutic footwear that off-loads the foot by at least $30 \%$ may be associated with lower risk of recurrence. ${ }^{[486]}$

\section{Diabetic foot infections}

Compared to non-diabetics, patients with diabetes are more susceptible to infections due to their impaired inflammatory response, inferior wound healing owing to inadequate phagocytic clearance, increased oxidative stress and down regulation of different growth factors resulting in defective angiogenesis. ${ }^{[496]}$ The Infectious Diseases Society of America (IDSA) recommends that the antibiotic regimen in patients with diabetic foot infections should be based upon culture and susceptibility analysis. ${ }^{[497]}$ Additional therapies may include the use of antibiotic impregnated beads, application of negative pressure wound therapy (NPWT) and hyperbaric oxygen [Figure 12]. ${ }^{[498]}$

In 2014, The Society for Vascular Surgery (SVS) published the Threatened Limb Classification System [Figure 13], based on 3 major risk factors associated with limb amputation: Wound, Ischemia and foot Infection (WIfI). ${ }^{[499]}$

Strategies aimed at preventing foot diseases are cost-effective and can even be costsaving if increased education and efforts 


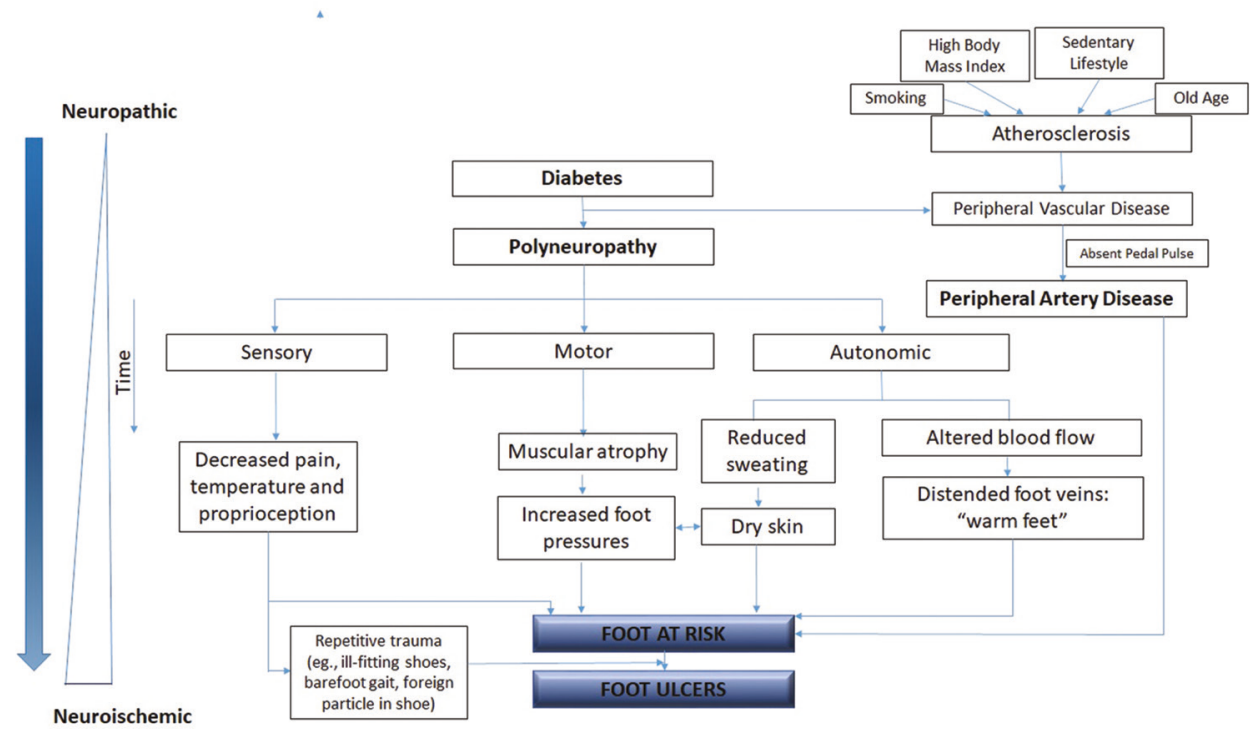

Figure 10: Pathogenesis of diabetic foot ulcer. Adapted from Boulton et al., 2018. ${ }^{[486]}$

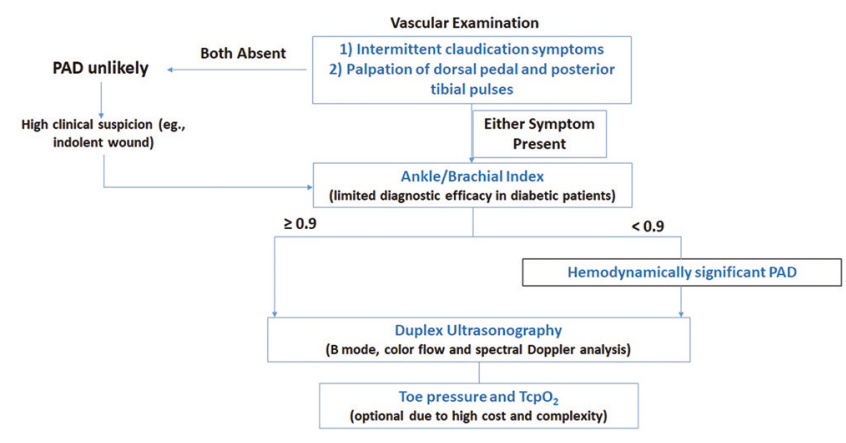

Figure 11: Vascular examination for PAD diagnosis. Adapted from Boulton et al., 2018;[486] PAD: Peripheral artery disease; Tcp02: Transcutaneous oximetry

are focused on those patients with recognized risk factors for the development of foot problem. The management of diabetic foot disease may seem poorly defined by comparison with complications such as nephropathy, hyperlipidaemia and retinopathy, for which clear guidelines exist. A multidisciplinary team approach, particularly in specialized diabetic foot clinics, can reduce the burden of diabetic foot complications in developing countries like India. Patients not conforming to the foot care advice suffer and develop new problems and/or require surgical procedures. ${ }^{[500]}$ Present guideline focuses on the various mechanisms of managing diabetic foot disease.

\section{Considerations}

Identifying a diabetic patient at-risk of a foot ulcer is an important step in the timely management of future

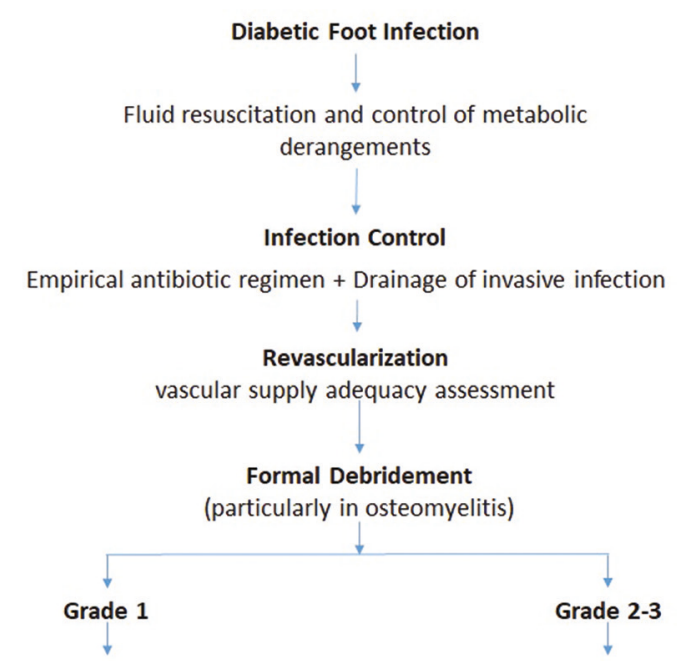

Appropriate dressing/offloading

Negative Wound Pressure Therapy

Figure 12: Diagnosis and management of diabetic foot infection. Adapted from Boulton et al., 2018. ${ }^{[486]}$ MDR0: Multi-drug Resistant Organism; NWPT: Negative Wound Pressure Therapy

complications. The panel recommended IWGDF 2019[501] risk stratification system for risk assessment and the corresponding frequency of foot screening and examination [Table 15].

The panel endorsed the IDF 2017 recommendations for diagnosis and management of diabetic foot complications. However, few of the recommendations were modified based on local factors such as limited resources and lack of quality assurance in laboratories, which were reviewed in an Indian context. 


\begin{tabular}{|c|c|c|c|}
\hline IWGDF risk category & Ulcer risk & Characteristics & Frequency (adapted for the Indian population) \\
\hline 0 & Very low & No LOPS and no PADS & Once every 12 months \\
\hline 1 & Low & LOPS or PAD & Once every 6-12 months \\
\hline 2 & Moderate & $\begin{array}{l}\text { LOPS + PAD or } \\
\text { LOPS + foot deformity } \\
\text { LOPS + foot deformity }\end{array}$ & Once every 3-6 months \\
\hline 3 & High & $\begin{array}{l}\text { LOPS or PAD + one or more of the following: } \\
\text { History of foot ulcer } \\
\text { A lower-extremity amputation (minor or major) } \\
\text { End-stage renal disease }\end{array}$ & $\begin{array}{l}\text { Once every 1-3 months } \\
\text { And at every visit to the doctor }\end{array}$ \\
\hline
\end{tabular}

Adapted from International Consensus on the Diabetic Foot 2019. ${ }^{[501]}$ LOPS: Loss of protective sensation, PAD: Peripheral artery disease

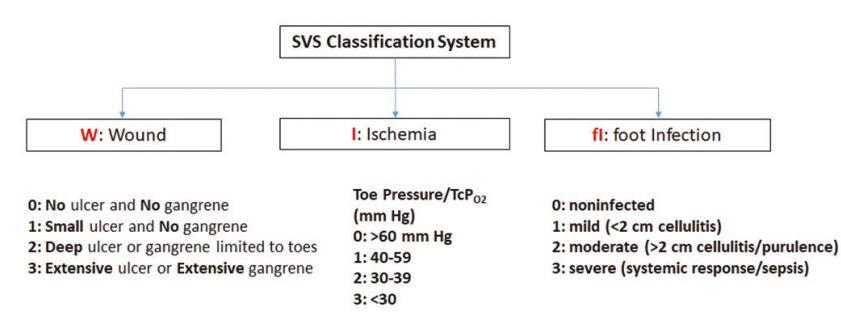

Figure 13: Threatened limb classification system. Adapted from Mills et al., 2014;[499] SVS: Society for vascular surgery

\section{Rationale and Evidence}

\section{Detection and timely screening}

- Vibration perception threshold (VPT) is considered as a gold standard for diagnosis of diabetic peripheral neuropathy. However, use of simple clinical scores such as NSS and diabetic neuropathy examination (DNE) scores were found to be simple and useful tools for the diagnosis of peripheral neuropathy in-patients with diabetes. ${ }^{[502,503]}$ Moreover, a good correlation between VPT score with tuning fork, monofilament and ankle reflex was found suggesting that simple bed side tests are useful in clinical practice, even in those subjects in whom foot care practices are not followed. ${ }^{[504,505]}$

- $\quad$ Using NSS and NDS in T2DM patients has been found to be a useful resource in evaluating diabetic sensorimotor polyneuropathy as an important bed side tool. ${ }^{[411,506,507]}$

- Graduated RydelSeiffer tuning fork has a high specificity and a fairly good sensitivity in the diagnosis of diabetic foot problems. ${ }^{[508]}$

- Tip-therm, a device which tests for temperature discrimination, was compared with two validated methods for detection of neuropathy-a monofilament and biothesiometry in a study comprising 910 diabetic patients. Tip-therm was found to be an inexpensive, highly sensitive and specific device for detection of diabetic neuropathy when compared with biothesiometry and a monofilament. ${ }^{[509]}$

- Evidence suggests that abnormal plantar foot pressure may exist in diabetic patients before there is evidence of neuropathy (determined by biothesiometry and monofilament tests). Podotrack, a novel, inexpensive method can be used as a screening test for abnormal plantar foot pressure in this patient population. ${ }^{[510]}$

- Gait variations and restrictions in subtalar and first metatarsophalangeal joint have been reported in cases of diabetic neuropathy even before the onset of foot deformity and could be used as an aid for early diagnosis. ${ }^{[511]}$

- $\quad \mathrm{ABI}$ and tcPO2 may be used as predictors of ulcer healing and amputation, respectively; $\mathrm{ABI}=0.6$ was found to have $100 \%$ sensitivity and $70 \%$ specificity and tcPO2 $=22.5$ was found to have $75 \%$ sensitivity and $100 \%$ specificity in predicting wound healing. ${ }^{[512]}$

\section{Avoid walking bare foot}

- Sociocultural practices like bare foot walking indoors and other religious places, use of improper footwear and lack of knowledge regarding foot-care are significant contributors of diabetic foot complications in India. ${ }^{[484,488,513]}$ Therefore, the panel emphasized on educating patients on problems associated with walking bare foot ${ }^{[500]}$ and advice on the use of appropriate/therapeutic footwear, particularly those at high-risk to prevent the development of foot deformities and ulceration. ${ }^{[514]}$

- A questionnaire-based study evaluating the foot care knowledge and practices with foot complications in 300 Indian patients suggests that majority of these patients were not educated previously about foot care and walked indoors without foot wear. The study emphasized that poor knowledge of foot care and poor footwear practices are important risk factors for foot problems in diabetes and called for a joint effort from doctors and footwear industry and to educate patients about foot care and improve their choice and selection of footwear so as to reduce foot problems. ${ }^{[515]}$

\section{Management of diabetic foot complications}

- MRI has emerged as the most accurate method of diagnosing bone infection, but bone biopsy for culture and histopathology remains the criterion standard. ${ }^{[516]}$

- Neuropathy increases the risk of amputation 1.7-fold; 12 -fold if there is deformity and 36-fold if there is 
a history of previous ulceration. Antioxidants have been shown to be effective in the prevention and treatment of neuropathy. Recent evidence suggests that physiological antioxidants like alpha-lipoic acid, along with chromium and inositol may be beneficial in reducing glycaemic load and replenishing myoinositol stores, respectively. ${ }^{[517]}$

- A phase 3 multicentre study has provided evidence to support the safety and efficacy of rhEGF formulated gel; the gel healed diabetic foot ulcers faster than treatment with placebo. ${ }^{[518]}$

- A peptide mimetic of the C-terminus of Cx43 (gapjunctional protein), alpha connexincarboxy-terminal (ACT1), when incorporated into the standard-of-care protocols, was found to be associated with a greater percentage of participants achieving 100\% ulcer reepitheliazation and a reduced median time-to-completeulcer closure. ${ }^{[519]}$

- Imipenem was found to be the most potential antimicrobial against both Gram Positive Cocci and Gram Negative Bacilli. Among combination therapies, cefipimetazobactum and cefoperazone-sulbactum were the most effective. Antimethicillin-resistant Staphylococcus aureus (MRSA) antimicrobials such as linezolid and vancomycin and anti-extended spectrum of beta-lactamase (ESBLs) like imipenem and meropenem can be given to patients producing MRSA or ESBL. ${ }^{[520]}$

\section{Adjunctive treatment options}

- In a small study comprising 6 patients with DFU, hyperbaric oxygen therapy showed a positive effect in initiating ulcer healing compared to standard treatments like offloading, wound debridement and glucose control. ${ }^{[521]}$

\section{Pressure off-loading}

- Pressure modulation commonly referred to as 'off-loading' is an important component in the management and treatment of diabetic foot ulcers. It involves mitigation of pressure at an area of high vertical or shear stress. ${ }^{[522,523]}$ Combining effective, easy to use off-loading devices such as total contact casts and removable cast walkers ensures patient compliance, heal foot ulcers and avert limb amputations. ${ }^{[523,524]}$

- Mandakini off-loading device ${ }^{[525,526]}$ and Samadhan offloading system ${ }^{[526,527]}$ were found to be most economical, easy to apply and effective methods to re-distribute the pressure in ulcerative areas.
- A recent systematic review and meta-analysis report that compared with standard dressing changes, negativepressure wound therapy had a higher rate of complete healing of ulcers (RR: $1.48 ; 95 \%$ CI: 1.24, 1.76; $\mathrm{p}<0.001$ ), shorter healing time (MD:-8.07; 95\% CI:-13.70,-2.45; $\mathrm{p}=0.005$ ), greater reduction in ulcer area (MD: $12.18 ; 95 \%$ CI: $8.50,15.86 ; \mathrm{p}<0.00001)$, greater reduction in ulcer depth (MD: 40.82; 95\% CI: 35.97, 45.67; $<<0.00001$ ), fewer amputations (RR: $0.31 ; 95 \%$ CI: $0.15,0.62 ; \mathrm{p}=0.001$ ) and no effect on the incidence of treatment-related adverse effects (RR, 1.12; 95\% CI: 0.66, 1.89; $\mathrm{p}=0.68) .{ }^{[528]}$

- The risk of amputation increases with increasing severity and location of the deformity and complexity/stage of Charcot neuroarthropathy, as per Roger's Charcot foot classification system. ${ }^{[529]}$

- Patients who use therapeutic footwear have demonstrated lower foot pressure, while those who use nontherapeutic footwear show an increased foot pressure, implying that therapeutic footwear is useful in reducing new ulceration and consequently the amputation rate in the diabetic population. ${ }^{[514]}$

- Patients with diabetic peripheral neuropathy and/ or prior foot ulcers report a higher incidence of falls compared to non-diabetics. ${ }^{[530-532]}$ Specialty offloading devices, along with decreased sensorimotor function, musculoskeletal/neuromuscular deficits and pharmacological complications are implicated as reasons for this high incidence observed. Novel technological advancements, such as virtual reality proprioceptive training, may help in reducing the risk of such falls. ${ }^{[531]}$

\section{IMPLEMENTATION}

Availability of basic equipment, appropriate protocols, structured records and recall systems need to be supported by appropriate training for professionals providing screening and management services. Standard care of diabetic foot complications [Annexure 4 and 5] includes maintaining adequate vascular supply, preventing and treating soft-tissue and bone infection, performing initial excisional debridement and maintenance debridement as inducted, adhering to high-quality off-loading. Liaison needs to be established with orthoptists, footwear suppliers and cast technicians. Multidisciplinary management programs should be initiated focusing on prevention, education, regular foot examinations, aggressive intervention and optimal use of therapeutic footwear. 


\section{Diabetes and Cardivascular Diseases}

\section{ReCOMmEndations}

\section{Recommended Care}

- Cardiovascular risk factors that should be assessed in all patients at diagnosis and annually including

- Dyslipidemia

- Hypertension

- Smoking status

- Family history of premature coronary disease

- Presence of albuminuria

- Body mass index (BMI) $\geq 25$

- Presence of hyperuricemia

- Current or previous CVD events, age, body weight, BP and pulse, of patients should be recorded during their first and subsequent visits

- UKPDS risk engine and QRISK3 are simple and effective tools for identifying and predicting CVD risks in patients with T2DM and should be recommended for identifying high risk individuals*

- Patients with diabetes and CVD risk should follow the ABC treatment goals**

- $\mathrm{A}(\mathrm{HbA} 1 \mathrm{c}):<7 \%$

- $\mathrm{B}(\mathrm{BP}):<130 / 80 \mathrm{mmHg}$

- $\mathrm{C}($ Cholesterol -LDL): $<100 \mathrm{mg} / \mathrm{dL}$

- All patients should be managed with lifestyle intervention including physical exercise and medical nutrition therapy

- Yoga has shown efficacy in improving the dyslipidemia state and lower BMI, in selected patients with T2DM.

- In high risk patients, low dose aspirin therapy should be administered along with lifestyle intervention

- Statins should be added to lifestyle intervention in all patients with CVD risk, if not contraindicated. The intensity can be modified or titrated according to patient's CVD risk, age, side-effects, tolerability, LDL-C levels etc.

- Glycemic control with glucose lowering drugs that are proven to be CV safe and beneficial should be recommended to reduce CVD risk and complications in patients with T2DM. SGLT2 inhibitors and GLP-1 receptor agonists are approved by various regulatory authorities for CV risk reductions, apart from their glucose lowering ability.

- Weight control should be an important consideration, while choosing glucose lowering therapy in overweight/obese persons

- Pharmacological antihypertensive therapy with subsequent titration in addition to lifestyle therapy should be initiated in patients with confirmed office-based BP of $>140 / 90 \mathrm{mmHg}$

- Pharmacological therapy for patients with diabetes and hypertension should comprise a regimen that includes ACE inhibitor/ARB, thiazide diuretics, calcium channel blockers, and selective $\beta$ blockers. If one class is not tolerated, it should be substituted with other class; however, FDCs of different drug classes may be preferred in patients with diabetes to reduce CVD risks and complications

- ACE inhibitors are the drug of choice for diabetes, if not contraindicated; and ARBs may be used if ACE inhibitors are not tolerated

- Other medications for dyslipidemia (fibrates, ezetimibe, concentrated omega-3 fatty acids, PCSK9 inhibitors) can be considered in patients failing to reach targets with conventional lipid lowering medications

*The treatment target goals should be individualized according to age, risk and comorbidity. **Risk factor: Low-density lipoprotein (LDL)-cholesterol $\geq 100$ $\mathrm{mg} / \mathrm{dL}$ (2.6 mmol/L), high blood pressure (> 140/90 mm Hg), smoking, overweight/obese, lack of physical activity

\section{Limited Care}

- Cardiovascular risk factors like albuminuria and hypertension should be assessed in all patients at diagnosis and annually

- Cardiovascular risk may be calculated by using different assessment tools for people with diabetes as recommended

\section{BACKGROUND}

Patients with T2DM are always at higher risk for several CVDs such as CAD, CHF, stroke, PAD, and cardiomyopathy. Furthermore, compared to patients without diabetes, T2DM patients have a considerably higher risk of $\mathrm{CV}$ morbidity and mortality. ${ }^{[533]}$ In addition, the coexistence of risk factors like hypertension, dyslipidemia, obesity and smoking with T2DM may increase the burden and complications of CVD ${ }^{[534]}$ In India, CVD attributes to nearly $25 \%$ of all deaths. Furthermore, according to the Global Burden of Disease study, age-standardized CVD mortality rate was 272 per 100000 population in India, which was higher than the global average of 235 per 100000 population. ${ }^{[35]}$ An Indian population-based study in 6198 patients with T2DM that evaluated the prevalence of CVD risk factors reported that, compared to participants with diabetes versus those without it, prevalence of hypertension was $73.1 \%$ (95\% CI: 67.2 to 75.0$)$ vs $26.5 \%$ (25.2 to 27.8 ), hypercholesterolemia was $41.4 \%$ ( 38.3 to 44.5 ) vs $14.7 \%$ (13.7 to 15.7$)$, hypertriglyceridemia was $71.0 \%$ (68.1 to 73.8 ) vs $30.2 \%$ (28.8 to 31.5 ), low HDL-C was $78.5 \%$ ( 75.9 to 80.1 ) vs $37.1 \%$ ( 35.7 to 38.5 ), and incidence of smoking/smokeless tobacco use was $26.6 \%$ (23.8 to 29.4 ) vs $14.4 \%(13.4$ to $15.4 ; p<0.001) .{ }^{[536]}$ Several landmark studies have reported that patients with T2DM are at increased risk for several cardiovascular complications. A brief overview of the CINDI studies, INTEHEART and INTERSTROKE is presented in Table 16..$^{[373,374,537,538]}$ 
Therefore, aggressive control of these risk factors may delay or reduce the incidence of CVDs in T2DM patients.

\section{Considerations}

When framing recommendations for diabetes and CV risk, following factors should be reviewed: hypertension, smoking, obesity, increased fasting insulin and IR, lifestyle intervention, atherogenic lipid profile (abnormal cholesterol, high triglycerides).

Primary prevention of CVDs aims at preventing patients from the event of CHD/CVD. This includes engaging in moderate physical activity, maintaining normal body weight, limiting alcohol consumption, reduction of sodium intake, maintaining adequate intake of potassium, and consumption of a diet rich in fruits, vegetables, and low-fat dairy products with less saturated and total fat. Secondary prevention of CVDs in patients with diabetes plans to reduce the mortality and morbidity and prevent the repeated CVD event. This comprises treatment with aspirin, $\beta$-blockers, ACE inhibitors and statin. The tertiary prevention intends at rehabilitation, preventing complications, and improving QoL. This can be achieved with some interventional surgical procedures. Quaternary prevention targets at preventing over diagnosis, over medicalization, over labelling and over treatment.

\section{Rationale and Evidence Identification}

Cardiovascular risk factors such as dyslipidemia, hypertension, smoking, high body-mass index (BMI), family history of premature coronary disease and the presence of albuminuria and hyperuricemia should be assessed at least annually in all patients with T2DM. ${ }^{[534,539,540]}$ Even the CINDI and CINDI 2 studies in Indian population recommend screening of $\mathrm{CV}$ complications at the time of diagnosis. ${ }^{[373,374]}$

The following tools have been used by several physicians for assessment of the CVD risk in individuals with diabetes and CVD.
- $\quad$ QRISK3 Risk Score ${ }^{[441,542]}$
- UKPDS Risk Engine. ${ }^{[543]}$

Recently, the DISCOVER observational study $(\mathrm{N}=15,992)$ has been initiated to collect realworld data from 38 countries to understand patterns of T2DM care in patients who initiated a second-line glucose-lowering therapy. Data from several lower-middle and upper-middle income countries will be collected for the first time through DISCOVER. This might help in preparing informed clinical guidelines and prepare effective healthcare policies globally. ${ }^{[544]}$

Table 16: Studies assessing cardiovascular risk factors

\begin{tabular}{|c|c|c|c|c|}
\hline Study characteristic & CINDI (India, 2014) & CINDI 2 (India, 2016) & INTERHEART (Global, 2004) & INTERSTROKE (Global, 2016) \\
\hline Study population & $\begin{array}{l}4600 \text { newly diagnosed } \\
\text { patients with T2DM } \\
(\text { men: } 67 \%)\end{array}$ & $\begin{array}{l}1500 \text { newly detected } \\
\text { young-onset diabetes } \\
\text { patients (men: } 74 \% \text { ) }\end{array}$ & $\begin{array}{l}15,152 \text { cases with acute MI, } \\
14,820 \text { controls from } 52 \\
\text { countries }\end{array}$ & $\begin{array}{l}13,447 \text { cases }(10,388 \text { with } \\
\text { ischemic stroke and } 3059 \\
\text { intracerebral hemorrhage) } \\
\text { and } 13,472 \text { controls from } 32 \\
\text { countries (men: } 59.6 \%)\end{array}$ \\
\hline Study objective & $\begin{array}{l}\text { To assess patients for } \\
\text { diabetic complications, } \\
\text { hypertension, } \\
\text { dyslipidemia, } \\
\text { BMI, diagnosis of } \\
\text { retinopathy, neuropathy } \\
\text { and nephropathy }\end{array}$ & $\begin{array}{l}\text { To evaluate patients for } \\
\text { complications of diabetes } \\
\text { and CV risk factors such } \\
\text { BMI, hypertension, } \\
\text { dyslipidemia, and smoking }\end{array}$ & $\begin{array}{l}\text { To assess relationship } \\
\text { between smoking, history } \\
\text { of hypertension or diabetes, } \\
\text { WHR, dietary patterns, } \\
\text { physical activity, consumption } \\
\text { of alcohol, blood Apo, and } \\
\text { psychosocial factors to MI }\end{array}$ & $\begin{array}{l}\text { To assess relationship between } \\
\text { stroke and its risk factors } \\
\text { including hypertension, physical } \\
\text { activity, ApoB/ApoA1 ratio, } \\
\text { diet, WHR, psychosocial } \\
\text { factors, current smoking, alcohol } \\
\text { consumption and diabetes }\end{array}$ \\
\hline Results overview & $\begin{array}{l}\text { Hypertension, obesity } \\
\text { and dyslipidemia were } \\
\text { present in } 23.3 \%, 26 \% \\
\text { and } 27 \% \text { patients, } \\
\text { respectively }\end{array}$ & $\begin{array}{l}\text { Hypertension, dyslipidemia, } \\
\text { BMI }>23 \mathrm{~kg} / \mathrm{m}^{2} \text {, and } \\
\text { smoking were present in } \\
27.6 \%, 62.4 \%, 84.2 \text { and } \\
24 \% \text { patients. Diabetic } \\
\text { retinopathy, neuropathy, } \\
\text { and nephropathy were seen } \\
\text { in } 5.1 \%, 13.2 \% \text {, and } 0.9 \% \text {. } \\
\text { Ischemic heart disease, } \\
\text { PVD, and stroke were } \\
\text { presented in } 0.7 \%, 2 \% \text {, and } \\
0.1 \% .95 .33 \% \text { needed statin } \\
\text { therapy }\end{array}$ & $\begin{array}{l}\text { Diabetes, along with } \\
\text { smoking raised ApoB/ } \\
\text { ApoA1 ratio, history of } \\
\text { hypertension, abdominal } \\
\text { obesity, psychosocial factor, } \\
\text { lack of daily consumption of } \\
\text { fruits and vegetables regular } \\
\text { alcohol consumption, and lack } \\
\text { of regular physical activity } \\
\text { were all significantly related } \\
\text { to acute myocardial infarction } \\
(P<0.0001 \text { for all risk factors } \\
\text { and) }\end{array}$ & $\begin{array}{l}\text { Previous history of hypertension } \\
\text { or BP of } 140 / 90 \text { mm Hg or } \\
\text { higher, regular physical activity, } \\
\text { WHR, psychosocial factors, } \\
\text { smoking, cardiac causes, } \\
\text { alcohol consumption and } \\
\text { DM were all associated with } \\
\text { stroke. Hypertension was more } \\
\text { associated with intracerebral } \\
\text { hemorrhage than with ischemic } \\
\text { stroke, whereas current smoking, } \\
\text { diabetes, Apo, and cardiac causes } \\
\text { were more associated with } \\
\text { ischemic stroke }(P<0.0001)\end{array}$ \\
\hline
\end{tabular}

DM: Diabetes mellitus, Apo: Apolipoproteins, BMI: Body mass index, MI: Myocardial infarction; T2DM: Type 2 DM, PVD: Peripheral vascular disease, WHR: Waist-to-hip ratio, BP: Blood pressure 


\section{Management \\ Lifestyle intervention}

Early identification of metabolic syndromes such as $\mathrm{AO}$, elevated BP, hypertriglyceridemia, reduced HDL cholesterol, borderline high-risk LDL cholesterol and IFG $(110$ to $126 \mathrm{mg} / \mathrm{dL}$ ) and design interventions to reduce the CVD risks are the major goals of the primary prevention. ${ }^{[545]}$ Furthermore, close monitoring and maintaining recommended targets for BP (130/80 mmHg), lipid control (LDL $<100 \mathrm{mg} / \mathrm{dL}$ ), and glycaemia $(\mathrm{A} 1 \mathrm{C}<7 \%)$ is important for the prevention of CVD in patients with T2DM. ${ }^{[545,546]}$ In addition, physical exercise, weight control, lifestyle modification with changing food habits, and cessation of smoking also prevents the CVD risk in T2DM patients. ${ }^{[545]}$

- Diet: In the PURE study $(\mathrm{N}=135,335)$, a diet rich in carbohydrates was shown to be associated with higher risk of total mortality. Surprisingly, both, total fat and individual fat type were not correlated with CVD, CVD-related mortality or MI. In fact, saturated fat had an inverse correlation to stroke. ${ }^{[547]}$ Therefore, a high carbohydrate intake is a potent risk factor of CVD and mortality. Current nutritional recommendations for patients with T2DM propose restricting the total carbohydrate intake to $\sim 45-50 \%$ of the total energy. Moreover, there is increased focus on the quality of carbohydrate intake, with an emphasis on including complex carbohydrates like brown rice and whole grain wheat into the diet. ${ }^{[548]}$

- Substitution of dietary saturated fat with PUFAs is reported to be associated with improved CV outcomes. Moreover, American Family Physicians (AFP) advocates that the Mediterranean diet can reduce $\mathrm{CV}$ mortality and the DASH eating plan is associated with a reduced risk of CHD ${ }^{\left[{ }^{[49]}\right.}$ Moreover, the following dietary adaptations can be made to lessen the development of CVDs in T2DM patients: reductions in caloric intake (by $500 \mathrm{kcal} /$ day to $800 \mathrm{kcal} /$ day), total fat intake (especially saturated fat) and food portion sizes, increased consumption of dietary fiber, and moderate alcohol use. ${ }^{[550]}$

- Physical activity: It is an independent and protective risk factor associated with reduced $\mathrm{CV}$ morbidity and mortality $(\mathrm{OR}, 0.86 ; \mathrm{p}<0.0001)$, and physical inactivity accounts for $12.2 \%$ of the population-attributable risk for acute MI and $6 \%$ of CHD with an estimated 0.68 year reduction in life expectancy. ${ }^{[549]}$ The exercisebased cardiac rehabilitation (CR) is the cornerstone for secondary prevention of CVD. CR is associated with a $13 \%$ and $26 \%$ lower all-cause and CVD mortality, respectively and a $31 \%$ reduction in hospital admissions at 12 months in patients with CHD. ${ }^{[549]}$ Hence, AFP guidelines recommend that physical activity for adults should be at least 150 minutes of moderate-intensity aerobic activity per week, 75 minutes of vigorousintensity aerobic activity per week, or an equivalent combination. ${ }^{[51]}$
- Yoga: A randomized parallel study in India compared efficacy of yoga in addition to OADs and reported a significant reduction in total cholesterol, triglycerides, LDL-C, and body weight in patients after yoga. ${ }^{[552]}$ Furthermore, evidence suggests that yoga also helps in reducing the blood glucose levels, ${ }^{[533-555]}$ lipid levels, ${ }^{[53,554]}$ body weight, and $\mathrm{BP}^{[554]}$ in patients with T2DM

- Stress management: Evidence state that psychosocial stress has an association with the etiology and pathogenesis of CVDs. ${ }^{[556]}$ Most notably, the INTERHEART and INTERSTOKE studies report that psychological factors have a strong effect towards MI (OR: 2.67, PAR 32.5\%, $\mathrm{p}<0.0001$ ) and ischemic stroke (OR: $2 \cdot 20,1 \cdot 78,2 \cdot 72$; $17 \cdot 4 \%, 13 \cdot 1,22 \cdot 6)$ respectively. ${ }^{[537,538]}$ In an RCT, cognitive behavioral therapy (CBT) had a $41 \%$ lower rate of fatal and non-fatal first recurrent CVD events (HR:0.59; $95 \%$ CI: $0.42,0.83 ; \mathrm{p}=0.002), 45 \%$ fewer recurrent acute MI (HR: $0.55,95 \%$ CI: $0.36,0.85 ; \mathrm{p}=0.007$ ), and a nonsignificant $28 \%$ lower all-cause mortality (HR: $0.72,95 \%$ CI: $0.40,1.30 ; p=0.28)$ than the reference group after adjustment for other outcome-affecting variables during a mean 94 months of follow-up period. ${ }^{[557]}$ Nonetheless, a recent Cochrane review did not find such associations of CVD events with the psychological interventions in CHD patients. ${ }^{[58]}$

\section{Pharmacological management}

- Medical treatment with pharmacotherapies like aspirin, lipid lowering drugs and BP controlling agents improves survival, extends QoL, reduces the need for intervention procedures, such as angioplasty and coronary artery bypass graft surgery, and decreases the incidence of subsequent MI. ${ }^{[59]}$

\section{Antiplatelet therapy}

- Aspirin is widely used for secondary prevention of CVD however; its use in primary prevention is still controversial. ${ }^{[534]}$ In the recent ASCEND study, aspirin use prevented serious vascular events in patients with diabetes with no evident cardiovascular disease at trial entry. However, these preventive benefits were counterbalanced with major bleeding hazards. ${ }^{[560]}$ Furthermore, a metaanalysis demonstrated $35 \%$ reduction in $\mathrm{MI}$ among men (RR: $0.65 ; 95 \%$ CI: $0.51,0.82 ; \mathrm{p}<0.01$ ), but the results were not significant in women (RR: $0.90 ; 95 \%$ CI: 0.71, 1.14; $\mathrm{p}=0.37) .{ }^{[561]}$ However, a systematic review including 10 RCTs reported no CVD benefit and trials with diabetes subgroup analyses also did not show any effect. ${ }^{[562]}$ Similarly, a recent meta-analysis evaluated aspirin for primary prevention of CVD in patients with diabetes and reported no difference with respect to the risk of allcause mortality (OR: 0.93, 95\% CI: 0.81, 1.06), individual atherosclerotic events, bleeding, gastrointestinal bleeding, or hemorrhagic stroke rates compared to placebo ${ }^{[563]}$ Furthermore, a meta-analysis $(n=4000)$ by the Antithrombotic Trialists' (ATT) collaborators showed 
that the effects of aspirin on major vascular events were similar for patients with or without diabetes: (RR: 0.88, 95\% CI: $0.67,1.15$ ) and (RR: $0.87,95 \%$ CI: $0.79,0.96$ ), respectively. ${ }^{[564]}$

- In patients with aspirin intolerance/allergy or patients at very high-risk for CVD, clopidogrel is recommended. ${ }^{[334,565]}$ Evidence suggests that clopidogrel was significantly more effective than aspirin in secondary prevention of CVD in patients with diabetes. ${ }^{[565]}$ Furthermore, dual antiplatelet therapy may be reasonable for up to a year after ACS. ${ }^{[534]}$

- A Cochrane systematic review report demonstrated that use of clopidogrel plus aspirin was associated with a reduction in the risk of $\mathrm{CV}$ events and an increased risk of bleeding compared with aspirin alone. However, only in patients with acute non-ST coronary syndrome, benefits outweigh harms. ${ }^{[566]}$

\section{Lipid lowering agents}

- A high prevalence of lipid abnormality in patients with T2DM positions them at high risk category in the CVD risk stratifications. Elevated levels of atherogenic cholesterol (AC), generally measured as non HDL-C, plays a central role in CVD, especially among Asian Indians. ${ }^{[567]}$

- For management of dyslipidemia, the primary goal is to reduce LDL-C levels to $<100 \mathrm{mg} / \mathrm{dL}$ by addition of drug therapy (statins) to maximal diet therapy. Furthermore, fibrates may be added if triglycerides remain $>200 \mathrm{mg} / \mathrm{dL}$ in patients receiving statin therapy. ${ }^{[559]}$ Statins reported a significant benefit in CV risk reduction and showed significant primary and secondary prevention of CVD/CAD deaths in patients with diabetes. ${ }^{[668-570]}$

- A recent meta-analysis investigating 4,351 diabetes patients reported that compared with placebo, standard-dose statin treatment resulted in a significant RRR of $15 \%$ in the occurrence of any major CV or cerebrovascular event (RR: $0.85,95 \%$ CI: 0.79, 0.91). Compared with standarddose statin treatment (simvastatin $20 \mathrm{mg}$, pravastatin $40 \mathrm{mg}$ or atorvastatin $10 \mathrm{mg}$ ), intensive-dose statin (simvastatin $80 \mathrm{mg}$ or atorvastatin $80 \mathrm{mg}$ ) treatment resulted in an additional 9\% RRR. ${ }^{[571]}$

- Moreover, statins were reported to produce similar results in various studies in India. ${ }^{[572,573]}$ Evidence advocates atorvastatin has negligible or no ability to increase HDL-C, which is the key feature in patients with diabetes. Thus, other statins should probably be preferred to atorvastatin in patients with diabetes/MS. ${ }^{[574]}$

- In addition ADA recommend that, either high intensity or moderate intensity statin therapy should be used together with lifestyle intervention according to patient age and ASCVD risk factors ${ }^{[2,534]}$ The details have been given in Annexure 6. The Lipid Association of India expert consensus statement 2016 revealed that statin therapy is highly effective in lowering NHDL-C, LDL-C, apolipoprotein $\mathrm{B}$, and remnant cholesterol, besides being remarkably safe ${ }^{[45]}$ Recent evidence shows a clear CVD benefit of lowering LDL-C with ezetimibe on top of a statin in patients with T2DM. $\cdot^{[575]}$

- Furthermore, in CHD/CHD risk-equivalent patients ezetimibe addition onto simvastatin, atorvastatin, or rosuvastatin provided greater LDL-C reductions and goal attainment than those who up-titrated these statin therapies. ${ }^{[576]}$ The Fenofibrate Intervention and Event Lowering in Diabetes (FIELD) study assessed the effect of fenofibrate on CV events in T2DM patients. Fenofibrate reduced total CV events, mainly due to fewer non-fatal MI and revascularizations but, did not significantly reduce the risk of coronary events such as CHD death or non-fatal MI. ${ }^{[577]}$

- Furthermore, USFDA states that the current evidence base is insufficient to support fibrates for CVD protection and that more trial evidence is needed. ${ }^{[578]}$ Nonetheless, prescribing lipid-lowering agents in older people with T2DM ( $>85$ years) requires special consideration because exposure to higher doses (or higher potency) might increase the risk of adverse effects instead of improving life expectancy.

- Proprotein convertase subtilisin/kexin type 9 (PCSK9) regulates degradation of LDL-receptors (LDLR). PCSK9 inhibitors, such as evolocumab and alirocumab, have been shown to enhance recirculation of LDLRs to the surface of hepatocyte cells and accelerate clearance of circulating LDL-C. PCSK9 inhibitors can prove to be a valuable treatment option for statin-intolerant patients. ${ }^{[579]}$

\section{Glucose lowering drugs}

- Intensive glycemic control with antidiabetic drugs [Table 1] reduces CV risk and complications in patients with T2DM. A meta-analysis including large, long-term prospective RCTs (such as the UKPDS, the prospective pioglitazone clinical trial in macrovascular events [PROactive], the Action in Diabetes and Vascular Disease: Preterax and Diamicron MR Controlled Evaluation [ADVANCE] trial, the Veterans Affairs Diabetes Trial [VADT] and the Action to Control Cardiovascular Risk in Diabetes [ACCORD] trial) report that intensive glycemic control was associated with $17 \%$ reduction in events of nonfatal MI (OR 0.83; 95\% CI: $0.75-0.93$ ), and a $15 \%$ reduction in events of CHD (OR, $0.85 ; 0.77-0.93)$; however the study did not find any significant effect on events of stroke $(0.93,0.81-1.06)$ or all-cause mortality $(1.02,0.87-1.19) .^{[275]}$

- DPP4, dipeptidyl peptidase 4; GIP, gastric inhibitory polypetide; GLP-1, glucagon-like peptide-1;SGLT2, sodium-glucose transport protein 2.

- In a meta-analysis of 301 clinical trials, the CVD risk of all glucose-lowering drugs including; metformin, sulphonylurea, thiazolidinedione, DPP4 inhibitor, AGI, SGLT2 inhibitors, GLP-1 analogue, meglitinides, and insulins, were evaluated. The results indicated that there were no significant differences in the association 
between any of the nine glucose-lowering drugs alone or in combination and risk of CV mortality. ${ }^{[580]}$

- Two SGLT2 inhibitors, empagliflozin and canagliflozin, were recently shown to provide CV benefits in patients with T2DM. Empagliflozin was reported to produce substantial reductions in CVD death (38\%) and all-cause mortality (32\%), as well as in hospitalization for $\mathrm{HF}$ $(35 \%)$, as compared with standard-of-care in EMPAREG OUTCOME trial. ${ }^{[205]}$ In the recently published CANVAS trial, canagliflozin significantly reduced the composite of death from CV causes, non-fatal MI, or nonfatal stroke (HR, $0.86 ; 95 \%$ CI, 0.75 to $0.97 ; p<0.001$ for non-inferiority; $\mathrm{p}=0.02$ for superiority) in T2DM patients with established CVD or at high risk for CV events. ${ }^{[207]}$

- Similarly in LEADER trial, liraglutide $1.8 \mathrm{mg}$ daily was associated with lower rates (patients) of death from CV causes $(4.7 \%$ vs. $6.0 \%$, HR, $0.78 ; 95 \%$ CI: 0.66 to 0.93 ; $\mathrm{p}=0.007)$ or any causes $(8.2 \%$ vs $9.6 \%, \mathrm{HR}, 0.85 ; 0.74$ to $0.97 ; \mathrm{p}=0.02$ ) compared to placebo in patients with T2DM. ${ }^{[237]}$ Therefore, using these medications early in the course of management in high risk T2DM patients could provide potential benefits from looming CVDs.

- Furthermore, recently released top-line results from the, CAROLINA trial (NCT01243424) showed that linagliptin was comparable to glimepiride vis-à-vis their impact on CV morbidity and mortality in patients with T2DM, after a median follow-up of 6.3 years. ${ }^{[581]}$

- In the SAVOR-TIMI 53 trial where patients were either randomized to saxagliptin or placebo, treatment with saxagliptin was associated with a $27 \%$ increased relative risk of hospitalization for heart failure in patients assigned to saxagliptin. ${ }^{[197]}$ The risk was highest in patients with elevated levels of natriuretic peptides, previous heart failure or chronic kidney disease. ${ }^{[582]}$

- Except saxagliptin, other DPP-4 inhibitors are cardiovascular neutral and therefore safe for the heart. ${ }^{[583]}$

- Major trials assessing the impact of glucose lowering agents in improving cardiovascular endpoints are schematically presented in Figure 14.

\section{Blood pressure lowering agents}

- A tight control of BP with pharmacological therapy like ARBs, ACE inhibitors, or $\beta$-blockers, diuretics, and calcium channel blockers helps in minimizing CVD risks in patients with T2DM.$^{[534]}$ Tight control of blood glucose decreases the risk of microvascular complications, whereas tight control of BP reduces both micro-and macrovascular complications.

- ADA, IDF and other organizations recommends a target BP of $130 / 80 \mathrm{mmHg}$ in diabetes patients. ${ }^{[232,534]}$ Furthermore, patients with confirmed office-based BP $>140 / 90 \mathrm{mmHg}$ in addition to lifestyle therapy should be initiated with pharmacological therapy to achieve BP goals. ${ }^{[534]}$

- A meta-analysis including 147 RCTs involving 464,164 people report a significant reduction in risk of coronary events (20-25\%) and stroke (30-45\%) with all five BP lowering agents. However, calcium channel blockers had a greater preventive effect on stroke (RR 0.92, 95\% CI: 0.85 to 0.98$).{ }^{[584]}$

- Two meta-analyses and ACCORD study reported that intensive BP control was associated with a reduction of stroke event, albeit with greater adverse effects. ${ }^{[350,585,586]}$ In addition, in the ADVANCE trial, a fixed combination of perindopril and indapamide was associated with mean reduction in SBP of $5.6 \mathrm{mmHg}$ and $\mathrm{DBP}$ of $2.2 \mathrm{~mm}$ of $\mathrm{Hg}$ after a mean of 4.3 years of follow-up in patients with T2DM. The relative risk of a major macrovascular or microvascular event was also reduced by $9 \% .{ }^{[285]}$

- Furthermore, some patients require a combination of two drugs in order to achieve a recommended BP target. Several Indian studies evaluated the efficacy of some FDCs: losartan $50 \mathrm{mg}$ plus ramipril $2.5 \mathrm{mg}$ vs each alone ${ }^{[587]}$ metoprolol extended release (XL) plus amlodipine vs losartan plus amlodipine, ${ }^{[588]}$ metoprolol and amlodipine, ${ }^{[589]}$ and reported that the FDCs were effective, safe and well-tolerated in patients with hypertension.

\section{Metabolic memory}

Metabolic memory refers to the beneficial effects of early, intensive control of hyperglycemia, which can help reduce chronic complications of T2DM in later years. The STENO-2, EMPA-REG and LEADER trials have shown the positive effects of metabolic memory. Recent results of STENO-2 trial after 21 years follow-up reported that an intensive, multifactorial intervention including ACE inhibitors/ARBs demonstrated a median of 7.9 years of gain of life in patients with T2DM. ${ }^{[591,592]}$ The choice of individual agent for a person with diabetes may be influenced by a number of factors including their risk profile ( $\mathrm{CV}$, renal, end-organ damage), preferences, and previous experience of therapy, as well as costs.

\section{IMPLEMENTATION}

Patients with diabetes and CVD risk should be assessed for complete lipid profile and BP measurement during their medical visits. Antiplatelet agents, lipid lowering therapies, and antihypertensive medications along with lifestyle interventions should be provided with individualization and preference of each patient. Structured annual assessment and record-keeping should be instituted. 


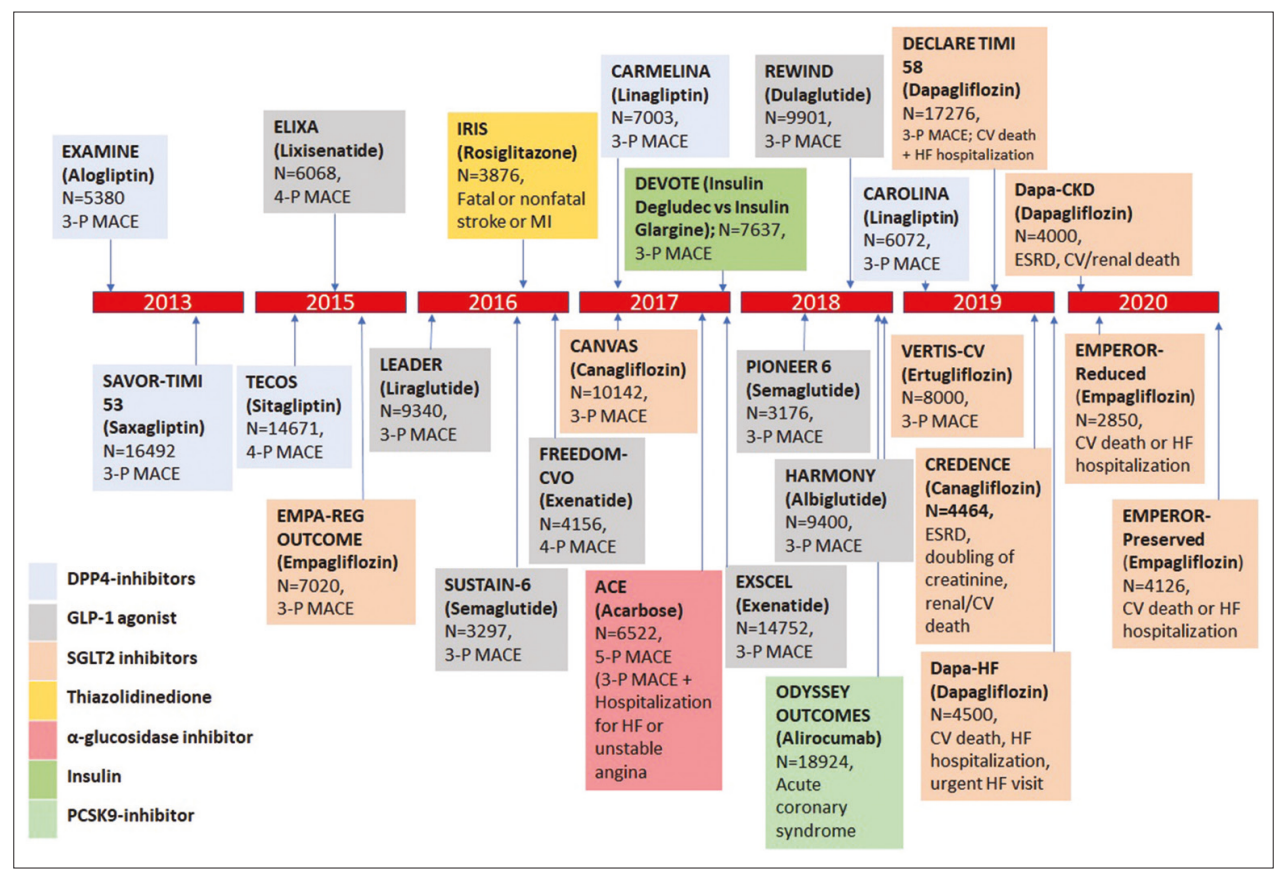

Figure 14: Major trials assessing cardiovascular outcomes in patients with diabetes. 3-P: 3-point; 4-P: 4-point; 5-P: 5-point. DECLARE-TIMI 58: Multicenter Trial to Evaluate the Effect of Dapagliflozin on the Incidence of Cardiovascular Events; ESRD: End-stage renal disease; HARMONY Outcomes: Effect of Albiglutide, When Added to Standard Blood Glucose Lowering Therapies, on Major Cardiovascular Events in Subjects With Type 2 Diabetes Mellitus; PIONEER 6: A Trial Investigating the Cardiovascular Safety of Oral Semaglutide in Subjects With Type 2 Diabetes; REWIND: Researching Cardiovascular Events With a Weekly Incretin in Diabetes; VERTIS CV: Cardiovascular Outcomes Following Ertugliflozin Treatment in Type 2 Diabetes Mellitus Participants With Vascular Disease. Adapted from Cefalu et al., 2018[590]

\section{Obesity and Type 2 Diabetes Mellitus}

\section{RECOMMENDATIONS}

\section{Recommended Care}

- The cut-off points for overweight and obesity in Indian T2DM patients are as follows:

- BMI $18-22.9 \mathrm{~kg} / \mathrm{m}^{2}$ : normal

- BMI 23-24.9 kg/m²: overweight

- $\mathrm{BMI} \geq 25 \mathrm{~kg} / \mathrm{m}^{2}$ : generalized obesity

- Waist circumference (WC) $\geq 90 \mathrm{~cm}$ for men and $\geq 80 \mathrm{~cm}$ for women: abdominal obesity

- Criteria for metabolic syndrome is as follows:

- Abdominal or central obesity (WC $\geq 90 \mathrm{~cm}$ for men and $\geq 80 \mathrm{~cm}$ for women) plus

- Any 2 of the following four factors:

- Increased triglycerides ( $\geq 150 \mathrm{mg} / \mathrm{dL}$ or specific treatment)

- Reduced HDL cholesterol (men: $<40 \mathrm{mg} / \mathrm{dL}$; Women: $<50 \mathrm{mg} / \mathrm{dL}$ or specific treatment)

- Increased blood pressure (systolic $\mathrm{BP} \geq 130$ or diastolic $\mathrm{BP} \geq 85 \mathrm{~mm} \mathrm{Hg}$ or treatment of previously diagnosed hypertension)

- Increased fasting plasma glucose ( $\mathrm{FPG} \geq 100 \mathrm{mg} / \mathrm{dL}$ or previously diagnosed $\mathrm{T} 2 \mathrm{DM}$ )

- Maintaining healthy lifestyle is recommended for management of metabolic syndrome.

- Moderate calorie restriction (to achieve a 5\%-10\% loss in body weight)

- $150 \mathrm{mins} /$ week of physical activity is recommended which includes aerobic activity, work related activity and muscle strengthening activity (to be increased to $300 \mathrm{mins} /$ week if tolerated).

- Change in dietary composition (low-calorie diet)

- Combination of aerobic and resistance training exercise

- Change in behavioural pattern

- Pharmacotherapy for obese patients with T2DM should be considered in addition to lifestyle changes in those with BMI $>25 \mathrm{~kg} / \mathrm{m}^{2}$

- GLP-1 analogues, DPP-4 inhibitors and SGLT2 inhibitors may be preferred as add-ons to metformin in obese T2DM patients 
- Lipase inhibitors (orlistat) may be used for inducing weight loss in addition to OADs in patients who have BMI $>25 \mathrm{~kg} / \mathrm{m}^{2}$

- Surgical treatment (bariatric surgery) may be considered as an option in diabetes patients with BMI $>32.5 \mathrm{~kg} / \mathrm{m}^{2} \mathrm{who}$ are not able to achieve durable

weight loss and improvement in co-morbidities including hyperglycemia despite reasonable nonsurgical management.

- Surgical options for weight loss surgery include

- Restrictive procedures: Laparoscopic adjustable gastric banding (LAGB) and sleeve gastrectomy

- Malabsorptive procedures: Bilio-pancreatic diversions (BPD)

- Combined procedures: Roux-en-Y gastric bypass (RYGB)

- Experimental procedures: Illeal interposition and duodeno-jejunal bypass, various implantable pulse generator

- Comprehensive lifestyle changes including dietary modification, exercise, behavioural management and pharmacotherapy, and bariatric surgery in select patients are the most effective interventions for weight management in T2DM patients

\section{BACKGROUND}

Obesity is a highly prevalent metabolic disorder that is often associated with T2DM. ${ }^{[134,593]}$ For adults, WHO defines overweight as BMI of $\geq 25 \mathrm{~kg} / \mathrm{m}^{2}$ and obesity as BMI of $\geq 30 \mathrm{~kg} / \mathrm{m}^{2} .{ }^{\left[{ }^{[54]}\right.}$ However, WHO and International Obesity Task Force (IOTF) suggested BMI cut-offs of 23 and $25 \mathrm{~kg} / \mathrm{m}^{2}$ for Asian Indian adults for overweight and obesity, respectively. ${ }^{[59,596]}$ Furthermore, the World Health Organization Asia Pacific Guidelines defined generalized obesity (GO, BMI $\geq 25 \mathrm{~kg} / \mathrm{m}^{2}$ ), AO (WC $\geq 90 \mathrm{~cm}$ for men and $\geq 80 \mathrm{~cm}$ for women) and combined obesity (CO, GO plus $\mathrm{AO})$ for Asian population. ${ }^{[595,597]}$ In India, the prevalence of obesity is rising at an alarming rate, especially affecting urban population. ${ }^{[134,598]}$ The ICMR-INDIAB study currently report that 135, 153 and 107 million individuals in India will have $\mathrm{GO}, \mathrm{AO}$ and $\mathrm{CO}$, respectively inextrapolation to the whole country. ${ }^{[597,599]}$ Furthermore, female gender, hypertension, diabetes, higher socio-economic status, physical inactivity and urban residence were significantly associated with obesity in Indian populations. Indians are at increased predisposition to diabetes that has been attributed to the "Asian Indian Phenotype" characterized by lesser GO as measured by BMI and greater central body obesity and more truncal fat as shown by greater WC and WHR. ${ }^{[4,134,600-603]}$ Abdominal obesity contributes significantly to metabolic alterations such as IR, dysglycemia and dyslipidaemia. ${ }^{[45,598,603-606]}$ T2DM is closely linked to obesity, particularly adult weight gain, and is the main contributor to rising healthcare costs. Whilst it seldom develops with BMI $<21 \mathrm{~kg} / \mathrm{m}^{2}$, most people with T2DM have a BMI $>25 \mathrm{~kg} / \mathrm{m}^{2}$ and around $50 \%$ have a BMI $>30 \mathrm{~kg} / \mathrm{m}^{2} .{ }^{[607]}$

High consumption of sugars among children and adults in India may also have clinical significance in view of the high tendency for Indians to develop IR, abdominal adiposity, and hepatic steatosis, and the increasing "epidemic" of T2DM. ${ }^{[17,134]}$ Because Asians Indians tend to develop diabetes at a significantly lower BMI and WC than white Europeans, lower thresholds of BMI to define overweight (BMI: $23-24.9 \mathrm{~kg} / \mathrm{m}^{2}$ ) and obesity (BMI $\geq 25 \mathrm{~kg} / \mathrm{m}^{2}$ ) were proposed by IDF and National Institute of Health and Care Excellence (NICE). ${ }^{[608,609]}$

In light of increasing prevalence of obesity in both developed and developing countries and a higher risk for developing IR, dyslipidemia, dysglycaemia and a higher CVrisk at lower levels of BMI in Indians, a consensus meeting was convened in New Delhi in $2008^{[10]}$ to redefine the cut-offs for BMI and WC for diagnosing overweight and obesity in Indian population. According to this consensus statement, a BMI of $18-22.9 \mathrm{~kg} /$ $\mathrm{m}^{2}$ should be considered as normal, a BMI of $23-24.9 \mathrm{~kg} /$ $\mathrm{m}^{2}$ should be considered as overweight, and BMI $\geq 25 \mathrm{~kg} / \mathrm{m}^{2}$ indicates presence of obesity. The upper limit for $\mathrm{WC}$ for men and women was defined as $90 \mathrm{~cm}$ and $80 \mathrm{~cm}$, respectively. ${ }^{[58]}$

\section{Considerations}

The following local factors were considered when framing recommendations for obesity that were reviewed in Indian context: high prevalence of obesity, high abdominal adiposity, increased fasting insulin and IR, nutritional factors, atherogenic lipid profile [increased triglycerides and LDL and low HDL]. ${ }^{[610]}$

\section{Identification of obesity in type $\mathbf{2}$ diabetes mellitus}

- At first and each subsequent visit, patients with T2DM should be screened for presence of excess body weight using appropriate anthropometric measurements (BMI, WC, WHR) and should be classified to as overweight or obese based on normal cut-off values recommended for Indian population.

- In Asian Indians, the BMI cut-off points to define overweight and obesity are lower than in other populations. ${ }^{[610-612]}$ Several investigators have shown that Asian Indians are more predisposed to develop insulin resistance and CVDs at lower levels of BMI as compared to other ethnic groups. ${ }^{[613,614]}$

- Based on the current evidence, WC is preferred over WHR as a measure of abdominal obesity with Asian Indian specific cut-offs. ${ }^{[610]}$ Asian Indians appear to have higher morbidity at lower cut-offs for WC than the western population; $\geq 90 \mathrm{~cm}$ in men and $\geq 80 \mathrm{~cm}$ in women. ${ }^{[604,610]}$

\section{Lifestyle intervention}

- Lifestyle interventions including diet therapy, physical activity, behavioural and psychosocial strategies have shown positive health outcomes in obese T2DM patients. The Diabetes Prevention Program (DPP) ${ }^{[615]}$ and the Look AHEAD (Action for Health in Diabetes) trial ${ }^{[616]}$ report clinically significant weight losses averaging $4-5 \%$ (or $4-5 \mathrm{~kg}$ ) at 3-4 years with lifestyle intervention. Similarly, a RCT including Asian Indians report that lifestyle intervention with less education lost a model-predicted $3.30 \mathrm{~kg}$ more in weight and $4.95 \mathrm{~cm}$ 
more in WC than those with more formal education. ${ }^{[617]}$

- The lifestyle interventions for overweight or obese T2DM patients should be based on the principle of decreased energy intake and increased energy expenditure to produce the negative energy balance. This includes low-calorie diet with higher intake of fibre, lower intake of saturated fats, optimal ratio of essential fatty acids, reduction in trans fatty acids, slightly higher protein intake, lower intake of salt, and restricted intake of sugar. ${ }^{[618]}$ High-protein meal replacement diet-based intervention in overweight/obese Asian Indians has shown significant reduction in weight, abdominal obesity, blood pressure, lipids, glycaemic parameters and hepatic enzymes compared with a standard control diet in Indians. ${ }^{[94]}$ Although studies assessing the ideal amount of carbohydrate intake for people with diabetes are inconclusive, modifying carbohydrate intake considering the blood glucose response is of value, especially in the Indian context, where carbohydrate intakes across all regions of India could be very high.

- Behavioural therapy should address modifiable factors such as eating patterns and exercise habits that can have significant impact on the management of obesity. A review in Indian scenario suggested that slow eating techniques along with stimulus control (not distracted by television, books, or other materials) have positive effect on weight loss. ${ }^{[619]}$ In obese T2DM patients, IDF recommends not only moderate calorie restriction, but also moderate increase in physical activity as a part of behavioural therapy in order to promote weight loss $\left(5-10 \%\right.$ loss of body weight in the first year) ${ }^{[620]}$ Other important components of behavioural therapy embrace self-monitoring, goal setting and stimulus or cue control. Such strategies help in setting up realistic goals, guide patients in identifying stimulus that lead to excessive nutrient intake and eliminate them accordingly. ${ }^{[621]}$

- Bodyweight has been shown to be inversely associated with physical activity. ${ }^{[622]}$ Patients with low physical activity have 3-fold greater risk of major weight gain in men and almost a 4-fold in women. ${ }^{[623,624]}$ Moreover, this association was stronger for women than for men and for obese compared to normal weight or overweight individuals. ${ }^{[625]}$ Furthermore, slow and prolonged exercise is associated with improved fat metabolism in muscle and better conservation of muscle mass during dietary restriction. ${ }^{[626,627]}$ An RCT comprising 262 sedentary men and women reported that combination of aerobic and resistance training exercise reduced WC from -1.9 to $-2.8 \mathrm{~cm}$ and mean fat mass of $-1.7(-2.3$ to $-1.1 \mathrm{~kg}$; $\mathrm{p}<0.05)$ compared with the non-exercise group. ${ }^{[628]}$ Physical activity, which includes aerobic activity, workrelated activity and muscle strengthening activity should be prescribed at the individual, community and societal level to help Asian Indians become more physically active [Table 17]. As per the WHO, the recommended levels of physical activity for adults (18-64 years) for at least 150 minutes of moderate-intensity weekly or 75 minutes of vigorous-intensity aerobic physical activity weekly. ${ }^{[629]}$

- In the Diabetes REmission Clinical Trial (DiRECT), 306 patients with T2DM, with BMI of $27-45 \mathrm{~kg} / \mathrm{m}^{2}$ and not receiving insulin were assessed for remission of T2DM during a primary care-led intensive weight management program. At 12 months, almost half of participants [68/149 (46\%)] achieved remission versus $4 \%$ in the control group, to a non-diabetic state and were off antidiabetic drugs. At 24 months, $64 \%$ of those who had lost more than 10 $\mathrm{kg}$, were still in remission. ${ }^{[630,631]}$

- The findings of the randomized controlled PREVIEW lifestyle intervention study reported that the total physical activity accounts for greater variance in IR and some related cardiometabolic risk factors as compared with moderate-to-vigorous physical activity. In adults with prediabetes, objectively measured physical activity and sedentary time have been found to have associated with cardiometabolic risk markers. ${ }^{[632,633]}$ Fixed low-energy diet has been shown to induce an overall $11 \%$ weight loss and showed significant improvements in insulin resistance; men appeared to benefit more than women. ${ }^{[634]}$

- Therefore the panel suggests that prescribing a combination of aerobic and resistance training exercises in individuals with T2DM can improve metabolic control while reducing obesity and its related complications.

\section{Pharmacotherapy for obese type 2 diabetes mellitus}

- Though lifestyle modifications are effective in inducing weight loss and improving diabetic status, they often fail requiring initiation of pharmacotherapy. Metformin is the

\begin{tabular}{|c|c|c|c|c|c|c|}
\hline $\begin{array}{l}\text { Type of physical } \\
\text { activity }\end{array}$ & $\begin{array}{l}\text { Moderate intensity } \\
\text { modality }\end{array}$ & Duration & $\begin{array}{c}\text { Frequency/ } \\
\text { days per week }\end{array}$ & $\begin{array}{l}\text { Vigorous intensity } \\
\text { modality repetitions }\end{array}$ & Duration & $\begin{array}{c}\text { Frequency/ } \\
\text { days per week }\end{array}$ \\
\hline $\begin{array}{l}\text { Aerobic physical } \\
\text { activity }\end{array}$ & $\begin{array}{l}\text { Brisk walking, stair } \\
\text { climbing, jogging ( } 4-7 \\
\mathrm{~m} / \mathrm{s} \text { ), cycling, treadmill } \\
\text { and swimming }\end{array}$ & $30 \mathrm{~min}$ & 5 & $\begin{array}{l}\text { Football, badminton, } \\
\text { basketball, running, rope } \\
\text { jumping, dancing }\end{array}$ & $20 \mathrm{~min}$ & 3 \\
\hline $\begin{array}{l}\text { Muscle } \\
\text { strengthening } \\
\text { activity }\end{array}$ & $\begin{array}{l}\text { Resistance weight training, } \\
\text { curls, presses, anti-gravity } \\
\text { exercise, isometric } \\
\text { exercise, children-body } \\
\text { weight activity (pull ups) }\end{array}$ & $\begin{array}{l}1-3 \text { sets of } 8-12 \\
\text { repetitions } \\
\text { targeting major } \\
\text { muscle groups }\end{array}$ & $2-3$ & $\begin{array}{l}\text { Resistance weight training, } \\
\text { curls, presses, anti-gravity } \\
\text { exercise, isometric } \\
\text { exercise, children-body } \\
\text { weight activity (pull ups) }\end{array}$ & $\begin{array}{l}>3 \text { sets of }>12 \\
\text { repetitions } \\
\text { targeting major } \\
\text { muscle groups }\end{array}$ & $2-3$ \\
\hline
\end{tabular}


first choice drug with some evidence for weight loss. ${ }^{[635,636]}$ The DPP and Indian Diabetes Prevention Program (IDPP) trial report that metformin and lifestyle intervention greatly reduce the risk of T2DM in overweight or obese patients. ${ }^{[637]}$ Use of GLP-1receptor agonists, ${ }^{[254]}$ SGLT-2 inhibitors, ${ }^{[255,638]}$ and to some extents DPP-4 inhibitors ${ }^{[257,258]}$ have been shown to induce weight loss and should be considered as add-on to metformin in obese T2DM patients. Furthermore, GLOBE study report that fixed dose combination (FDC) of acarbose/metformin in Indian T2DM patients was associated with significant reduction in body weight $(-1.7 \pm 2.2 \mathrm{~kg}$ compared to control $(\mathrm{p}<0.0001)) \cdot{ }^{[639]}$ Current ADA recommendations suggest SGLT2 inhibitors and GLP-1 agonists as preferred add-on agents in addition to lifestyle interventions and metformin for diabetes patients with compelling indications for weight loss. ${ }^{[251]}$

- Orlistat (tetrahydrolipstatin), a lipase inhibitor, is an approved agent for weight loss in India. It causes modest weight loss by blocking fat absorption from gut, and when used in combination with lifestyle changes was found to be effective in inducing weight loss as well as for prevention of diabetes. ${ }^{[600]} \mathrm{A}$ recent systematic review and meta-analysis report that treatment with orlistat and lifestyle intervention resulted in significantly greater weight loss $(p<0.001)$ and improved glycaemic control $(\mathrm{p}<0.001)$ in overweight and obese T2DM patients compared with lifestyle intervention alone. ${ }^{[640]}$

- Lorcaserin, an FDA approved agent for weight loss, has been recently approved in India. Lorcaserin is a highly selective serotonin $2 \mathrm{C}$ receptor agonist and acts on proopiomelanocortin neurons to induce satiety. It has been shown to cause meaningful weight loss and prevent progression to T2DM in patients with obesity. In the BLOOM-DM study, treatment with lorcaserin was associated with significant weight loss $(\geq 5 \%$ body weight) and improvement in glycaemic control in patients with T2DM. ${ }^{[641]}$ Results of the CAMELLIA-TIMI 61, CVOT with lorcaserin, suggested that durable weight loss observed with lorcaserin and lifestyle interventions was associated with significant reduction in the incidence of diabetes, improvement in the proportion of prediabetic patients not progressing to diabetes along with and significant reductions in the risk of a composite outcome for diabetic microvascular complications. ${ }^{[642]}$ There was no increase in 3 point MACE or extended MACE in patients treated with lorcaserin showing its CV safety. Lorcaserin facilitated the sustained weight loss without a higher rate of major cardiovascular events. ${ }^{[643]}$ However, the USFDA has issued a request to withdraw lorcaserin from the markets due to increased occurrence of cancer, based on a recent long-term safety study. ${ }^{[64]}$

\section{Surgery}

- The surgical options for weight loss include LAGB and sleeve gastrectomy, RYGB, BPD, illeal interposition and duodenojejunal bypass, and various implantable pulse generators. ${ }^{[610]}$

- Surgical treatment (bariatric surgery) is indicated in patients with BMI $>32.5 \mathrm{~kg} / \mathrm{m}^{2}$ with co-morbidity, or BMI $>37.5 \mathrm{~kg} / \mathrm{m}^{2}$ without co-morbidity; who fail to lose weight with medical management, ${ }^{[610]}$ although hard evidence for this is lacking. Evidence from several studies suggests that bariatric surgery provides durable glycaemic control compared with intensive medical therapy. ${ }^{[645-648]}$ Moreover, gastric bypass has been observed to uniquely restore the pancreatic $\beta$-cell function and reduce truncal fat, thus reversing the core defects in diabetes. ${ }^{[645]} \mathrm{In}$ addition, a systematic review and meta-analysis of RCTs report that RYGB surgery is superior to medical treatment for short-to medium-term remission of T2DM, and improvement of metabolic condition and $\mathrm{CV}$ risk factors. ${ }^{[649]}$

- Bariatric surgery is an effective option for severely obese patients with poorly controlled T2DM and weight loss due to gastric bypass surgery is associated with good glycaemic control. In patients who had undergone bariatric surgery, about $8 \%$ showed complete remission of diabetes while more than $90 \%$ showed a significant decrease in their insulin or OADs requirement. ${ }^{[650]}$

- Laparoscopic sleeve surgery and RYBG was found to be safe and effective treatment option among obese Indian population with T2DMwith significant remission rates $(>95 \%, \mathrm{p}<0.001)$, larger reductions in $\mathrm{HbAlc}$, and diabetes medication usage. ${ }^{[651-653]}$

\section{Medical devices for weight loss and weight management}

- Several minimally invasive medical devices have been recently approved by the FDA for short-term weight loss or management which can be used for obesity management in T2DM patients. ${ }^{[654]}$

- At present, there are four types of FDA-regulated devices intended for weight-loss:

- Gastric band which can be placed around the top portion of the stomach, thereby leaving a small portion available for food

- Electrical stimulation systems block nerve activity between the brain and stomach using electrical stimulators which are placed in the abdomen

- Gastric balloon systems act by delay gastric emptying using inflatable balloons which are placed in the stomach to utilize space.

- Gastric emptying systems drain food after eating with the help of a tube that is inserted between the stomach and outside of abdomen. 
There are two types of FDA-regulated devices intended for weight-loss:

- Oral removable palatal space occupying device which is worn during meals to limit bite size and the ingested, transient, space occupying device-an ingested material that transiently occupies space in the stomach

- However, high cost, extremely limited insurance coverage, and paucity of data limit their use. ${ }^{[655]}$

\section{Obesity and type 2 diabetes mellitus: increased risk of cancer}

- Individuals with obesity and T2DM are at greater risk of developing multiple cancers including breast, prostate, colorectal, gastric, pancreatic, hepatic. ${ }^{[656]}$ There are multiple potential metabolic abnormalities that occur in obesity and T2DM that may explain the increased risk of cancer and cancer-related mortality in these patients. ${ }^{[677-659]}$

- Currently, there are no specific guidelines for cancer screening in patients with obesity and T2DM, despite their higher risk of cancer. Further studies should be performed to determine whether specific screening strategies to target this population would lead to a decrease in their cancer mortality. Till further evidence, a high index of suspicion should be maintained.

\title{
InFECTIONS AND VaCCINATIONS
}

\section{ReCOMMENDATIONS}

\section{Recommended Care} \\ - All diabetes subjects should be educated about administering at least pneumococcal and influenza vaccine. \\ - Vaccination against pneumococcal disease, including pneumococcal pneumonia, with 13-valent pneumococcal conjugate vaccine (PCV13) is \\ recommended for children before age 2 years. \\ - People with diabetes aged 2 through 64 years should receive 23 -valent pneumococcal polysaccharide vaccine (PPSV23). At age $\geq 65$ years, regardless of \\ vaccination history, additional PPSV23 vaccination is necessary. \\ - Annual vaccination against influenza is recommended for all people $\geq 6$ months of age, especially those with diabetes. \\ - Quadrivalent influenza vaccine should be preferred. \\ - Vaccination is contraindicated/postponed in patients with: \\ - hypersensitivity to the active substances or to any of the excipients of the vaccine \\ - history of chicken egg allergy particularly when considering flu shot \\ - recent history of Guillain-Barre syndrome within six weeks of a previous influenza vaccination in the case of flu shot \\ - postponed in patients with febrile illness or any acute infection
}

\section{Limited Care}

The principles for infections and vaccinations during diabetes are as for recommended care subject to availability and affordability of pneumococcal and influenza vaccines.

\section{BACKGROUND}

The risk of developing infectious diseases due to diabetes is now being considered an important complication of diabetes. ${ }^{[1,2]}$ Diabetes increases the risk of infection by two to three times in comparison to the non-diabetic population. The morbidity and mortality associated with infectious diseases such as influenza, pneumonia and hepatitis, which is usually preventable by appropriate vaccination, also appears to be very high in diabetes subjects. ${ }^{[3]}$ Patients with T2DM, especially those with PVD, are at high risk for many types of typical and atypical infections due to immune dysfunction, DN and poor circulation. ${ }^{[4]}$ Furthermore, skin breakdown in patients with advanced diabetes and PVD provides a portal of entry for bacteria. Longer duration of diabetes and poor glycaemic control causes increased risk of pneumonia related hospitalizations in diabetes subjects due to compromised immune system of the host. ${ }^{[5]}$ A recent study demonstrated that patients with high blood glucose level are at increased risk of community-acquired pneumonia ${ }^{[6,7]}$ Even certain viral infections can lead to new onset of diabetes in the population who are genetically prone to develop diabetes.

\section{Considerations}

The decision about conducting a screening program should be based on local factors such as limited resources and high prevalence of diabetes related infections factors that were reviewed in Indian context.

\section{Rationale and Evidence}

\section{Infections in diabetes}

- Several factors have been implicated for infections in diabetes, of which, altered immunity is the most predominant one ${ }^{[4,8]}$ Other predisposing factors 
increasing susceptibility to infections include diabetes related complications, frequent catheterization and dialysis in chronic renal failure patients. Evidence that these immunological defects can be corrected through good glycaemic control support the importance of close monitoring of infectious diseases in subjects with diabetes. ${ }^{[9]}$

- Urinary tract, respiratory tract, foot and deep soft infections are most common in T2DM occurring with increased incidence and resulting in high mortality. ${ }^{[10,11]}$

- Following section deals with evidences from Indian and global studies on infections that commonly occur in patients with diabetes

- Influenza: Diabetes increases the risk of hospitalization after influenza infection and quadruples the risk of intensive care unit (ICU) admission after hospitalization. ${ }^{[12]}$ Death rates among patients with diabetes during influenza epidemics may increase up to $5-15 \% \cdot{ }^{[13]}$ Evidence that influenza can trigger coronary complications, when taken in the context of diabetes subjects, gains more significance since the risk for CVD is already 2-to 4-fold higher in this sub group. ${ }^{[11,14]}$

- Retroviral infections: Cirrhosis of liver in diabetes patients results in higher incidence of glucose intolerance (60-96\%) and overt diabetes $(20-60 \%) \cdot{ }^{[1]}$ Elevated rates of inflammation and endothelial cell dysfunction are observed in human immuno-deficiency virus (HIV) infected patients with T2DM. ${ }^{[15]}$ Moreover, in HIV patients undergoing active retroviral therapy, autoimmune diabetes may be caused due to protein inhibitors and nucleoside analogues. Therefore, in HIV patients with compensated cirrhosis and high IR, insulin should be the preferred choice of treatment. ${ }^{[1]}$

- Malignant otitis externa: Commonly occurs in patients with diabetes and is mostly caused by Pseudomonas aeruginosa. ${ }^{[16]}$ It can be prevented by creating proper awareness regarding healthy ear cleaning practices like, not using commercially available ear buds and other foreign objects or unsterilized cotton. Management protocol comprises of strict glycaemic control, correction of electrolyte imbalance, improvement in immunocompetence, aural toileting, hyperbaric oxygen therapy and prolonged systemic and ototopic antimicrobial therapy (3-6 weeks) with agents such as piperacillin with tazobactam, ciprofloxacin and cefoperazone. ${ }^{[16]}$

- Infections of hand and upper limb: Diabetes ulcers in the upper limb should be promptly treated with adequate surgical means in order to prevent spreading of infection. Creating awareness on healthy cleaning practices minimizes disability and result in better outcome. ${ }^{[17]}$

- UTIs: These, mostly asymptomatic bacterial infections, occur more frequently in female diabetes patients. In all hospitalized diabetes patients it is recommended to perform urine culture to detect presence of bacteriuria, a condition leading to an unexplained worsening of the glycaemic control in some patients. ${ }^{[18]}$

- Hepatitis: It has been observed that several patients with underlying diabetes suffer from prolonged or complicated course of acute viral hepatitis. It is possible that with impaired hepatocyte regenerating capacity, these patients run a more prolonged and complicated course. In diabetes population, hepatitis $\mathrm{B}$ and $\mathrm{C}$ produces more comorbidities and prolonged infections.

- Even though hepatitis B virus (HBV) itself may not cause diabetes directly, cirrhosis derived from HBV infection poses two fold higher risk for T2DM. ${ }^{[19]}$ Infection due to HBV may occur during monitoring of blood glucose and other procedures involving multi-patient use of finger stick devices designed for single-patient use and inadequate disinfection and cleaning of blood glucose monitors between patients. ${ }^{[20]}$

- When hepatitis $\mathrm{C}$ virus (HCV) infection occurs in diabetes patients, the chronicity as well as risk of infections further increases. In a meta-analysis of 22 studies, it was found that patients with T2DM were at higher risk for acquiring $\mathrm{HCV}$ than non T2DM patients (OR: 53.50, 95\% CI: 52.54, 54.82). ${ }^{[21]}$

- Hepatitis A is the most common vaccinepreventable virus acquired during travel and it is highly prevalent in the Indian subcontinent. Protection with hepatitis A vaccination is proven to last at least 15 years. ${ }^{[22]}$

- Tuberculosis: Because diabetes impairs host defense mechanism, it has long been known to be a risk factor for active tuberculosis (TB) and reactivation of latent TB. ${ }^{[23]}$ Evidence suggests that the risk of developing TB is increased among patients with diabetes, particularly during the first year after diagnosis of diabetes. ${ }^{[24]}$ Furthermore, it is associated with worse treatment outcomes, higher rates of relapse and higher mortality rates in patients affected by both diseases. It is estimated that $15 \%$ of TB cases globally could be attributed to diabetes and $40 \%$ of these cases are from India and China. ${ }^{[25]}$ Moreover, in developing Asian countries prevalence of TB among diabetes patients was 1.8-9.5 times higher than in the general population. ${ }^{[25]}$ The situation is particularly challenging in low-income and middle-income countries where TB is endemic. 
Data from a systematic review of 13 observational studies indicate that efforts to diagnose, detect, and treat diabetes early may have a beneficial impact on TB control. ${ }^{[26]}$

\section{Types of vaccines}

Various types of vaccinations recommended to prevent these infections are:

- Pneumococcal vaccination: Two pneumococcal vaccines are available: PPSV23 and PCV13. Secondary immune response after PCV13 immunization is higher, whereas response is lower after immunization with PPSV23 vaccine. ${ }^{[27]}$

- The panel recommends the use of PCV13 for adults' $\geq 50$ years followed by a dose of PPSV23 at least 1 year later (and at least 5 years after their previous PPSV23 dose) depending on the clinical judgement of the physician. These recommendations are in line with the guidelines from the ADA 2017, and are also in synergy with the guidelines released recently by the Indian Society of Nephrology 2016, Indian Academy of Allergy 2017, and the Geriatric Society of India 2015. ${ }^{[28-31]}$

- PCV13 is available for vaccination of older adults and must be considered an important step for vaccinating older diabetes patients with age of $>50$ years. PPSV23 may be offered to immune-compromised patients with diabetes for additional coverage after PCV13. Repeated vaccination with PPSV23 must be avoided to prevent hypo-responsiveness. Clinical judgment in relation to individual subjects should be relied upon before these recommendations are put into practice.

- Influenza vaccination: In all patients with T2DM with age $\geq 6$ months, excluding those who are allergic to eggs, influenza vaccine is recommended. ${ }^{[32,33]}$ Influenza vaccination among diabetes patients reduced hospital admissions by $79 \%$ in two influenza epidemics in England. ${ }^{[34]}$

- HBV: To all unvaccinated patients with diabetes of age 19-59 years, 3 dose series of $\mathrm{HBV}$ is recommended. ${ }^{[33]} \mathrm{In}$ unvaccinated patients with $\geq 60$ years of age, three dose series vaccine could be considered. ${ }^{[33]}$

- Apart from the vaccines mentioned above, other routinely recommended, age-related vaccines should also be provided to all diabetes patients. ${ }^{[33]}$

- Annexure 7 provides brief information on recommended vaccines for patients with diabetes.

\section{Methods to improve rate of vaccination}

- Despite the importance of vaccination in diabetes patients, vaccination rates are low in them. In a survey on 307 diabetes patients in Singapore, only $30.6 \%$ of patients were found to be vaccinated with influenza vaccine. ${ }^{[35]}$ Another cross-sectional survey on 279 diabetes patients in Spain determined the vaccination rates for seasonal influenza, pneumococcus and hepatitis $\mathrm{B}$ as $40 \%, 2 \%$ and $2 \%$ respectively. ${ }^{[36]} \mathrm{A}$ survey on 274 elderly people in Turkey revealed that the proportion of diabetes patients vaccinated for influenza or pneumococcus or tetanus as $38.1 \%, 13.4 \%$ and $9.28 \%$ respectively. ${ }^{[37]}$

- Perception, knowledge, and misconception that vaccines are infective and cause side effects are some of the barriers for avoiding vaccination. ${ }^{[35,36]}$

- Maintaining a diabetes registry, systemic tracking system, and reminder system serve as tools for improvising the acceptance to vaccination and communicating with the subjects for the need of vaccination which provides awareness on immunization. [36,38] The combined used of patient outreach letters, special immunization clinics, standing orders, and practitioner reminders on medical records resulted in a remarkable 15 fold increase in pneumococcal vaccinations in diabetes patients in Guam, United States. ${ }^{[39]}$ Similarly, a combination of strategies including dissemination of guidelines, advice on setting up disease and vaccine registers, call and recall systems and benchmarking of performance remarkably improved influenza and pneumococcal vaccination rates in high-risk individual groups including diabetes patients in the United Kingdom. ${ }^{[40]}$ Periodic training of the staff accompanied by ongoing assessment of immunization rates and work flow and also a close follow up with the patient or his care giver by the treatment team is beneficial in minimizing the risk of inappropriate re-vaccinations. ${ }^{[41]}$

- The protocols should also aim at implementing a quality assurance process so that the standards of care are maintained. ${ }^{[42]}$

\section{IMPLEMENTATION}

Apart from the micro-and macro-vascular events in diabetes, infections due to influenza and pneumococci should be considered as significant public health concern. All clinics providing vaccinations shall maintain the records to assess the efficacy of vaccines regarding occurrence of various complications in vaccinated individuals compared to nonvaccinated subjects. Vaccination strategies in diabetes should evolve as part of routine care and a central registry need to be maintained. 


\section{Sexual Dysfunction}

\section{ReCOMMENDATIONS}

\section{Recommended Care}

- A detailed history and examination should be conducted in an unintimidating private setting with structured interviews by encouraging discussion regarding sexual concerns in both men and women with diabetes.

- Appropriate language considering the patient's age and culture should be used to make the patient comfortable.

- Psychological and social disturbances if any, should be discussed in an empathetic manner.

- Promotion of lifestyle changes to reduce the associated risk factors should be encouraged in patients with diabetes of both sexes.

Men

- Adult men with diabetes should be screened with a detailed sexual function history for ED as early as when they are diagnosed with diabetes.

- Detection of ED and evaluation of the response to treatment should be performed by validated questionnaires such as IIEF or Sexual Health Inventory for men.

- PDE-5 inhibitors may be offered as a first-line therapy for the treatment of ED in men with diabetes as they improve the quality of life of the patients and are associated with low side-effects.

- Symptoms of hypogonadism including lack of interest in sex and ED should be investigated further with a screening for serum testosterone concentration in the morning. Testosterone replacement may be beneficial in men with diabetes with symptomatic hypogonadism.

Women

- To identify whether a diabetic woman has sexual dysfunction, eliciting a detailed history in a compassionate manner and examination is the first step.

- Several self-reported validated questionnaires such as Female Sexual Function Index, the Female Sexual Distress (FSD) Scale, the Brief Index of Sexual Functioning for Women, and the Derogatis Interview for Sexual Function have been developed to assess FSD.

- Currently, the therapeutic recommendations for FSD include maintaining a healthy lifestyle, achieving an optimal glycemic control, genitourinary infection control, resolving psychosocial issues.

- Treatment with water-based vaginal lubricants, hormone replacement therapy, clitoral therapy device, genital infection control therapy are recommended.

- Treatment strategies with dehydroepiandrosterone supplementation, estrogen or androgen replacement, flibanserin (serotonin $1 \mathrm{~A}$ receptor agonist and a serotonin 2A receptor antagonist) and PDE-5 inhibitors are investigated, however, currently there is limited evidence for their use.

\section{Limited Care}

- Adult men with diabetes should be screened with a detailed sexual function history for ED, as early as when they are diagnosed with diabetes.

- Symptoms of hypogonadism including lack of interest in sex and ED should be investigated further with a screening for serum testosterone concentration in the morning.

- Promotion of lifestyle changes to reduce the associated risk factors should be encouraged in men with diabetes and SD

- To identify whether a woman with diabetes has sexual dysfunction, a detailed history and examination is the first step.

- Currently, the therapeutic recommendations for FSD include maintaining a healthy lifestyle, achieving an optimal glycemic control, genitourinary infection control, resolving psychosocial issues.

\section{BACKGROUND}

Diabetes ensued vasculopathy and neuropathy has been associated with dysfuntion of normal sexual function leading to psychosocial disruption and decreased quality of life in both men and women. ${ }^{[600-662]}$ Sexual dysfunction (SD) in diabetics is a neglected aspect in India, primarily due to minimal communication time between physician and patient, lack of privacy during doctor visits and the taboo factor. In men with diabetes, erectile dysfunction (ED) as a result of autonomic neuropathy is commonly observed and the prevalence odds as compared with controls is more than 3.5 times ${ }^{[663]}$ In a study conducted in a hospital in New Delhi, Sondhi et al. observed the prevalence of ED to be $78.7 \%$ in men with T2DM versus $46 \%$ in non-diabetics and a significant correlation between duration of diabetes and ED ${ }^{[664]}$ Furthermore, the Massachusetts male aging study demonstrated that the risk of ED is double in aged diabetics versus the general population. ${ }^{\left[{ }^{665]}\right.}$ Diabetic neuropathy, impaired relaxation of cavernosal smooth muscle due to altered cyclic guanosine monophosphate/nitric oxide pathway and risk of decreased testosterone levels resulting from hypogonadism can constitute the underlying pathology of ED. ${ }^{[666,667]}$ Other sexual complications in men with diabetes include ejaculatory dysfunction and hypogonadism. The recent ADA guideline recommends testing for serum testosterone concentration in men with diabetes who have symptoms of hypogonadism. ${ }^{[668]}$

Compared with men, SD in women with diabetes is rarely investigated and most often untreated. Particularly in countries like India where gender inequality and cultural disparity is high, management strategies for tackling such health concerns are almost nonexistent. ${ }^{[699]}$ However, the findings of a meta-analysis showed that the risk of female sexual dysfunction (FSD) was two times higher (OR [95\%CI], $2.02[1.49,2.72])$ and correlated with a low Female Sexual Function Index (FSFI) score in women with diabetes as compared with nondiabetics. ${ }^{[670]} \mathrm{FSD}$ is an intricate condition 
involving both physiological and psychosocial changes and includes hypoactive sexual desire disorder, arousal and lubrication disorder, pain during sexual intercourse, and loss of ability to achieve orgasm. ${ }^{[671]}$ Hyperglycemia decreases the hydration of the vaginal mucosa and lubrication of the vagina, and is the cause of genitourinary infections and dyspareunia. The vascular complications and endothelial dysfunction may impact blood supply to clitoris and lead to poor lubrication of the vagina, and reduced arousal and dyspareunia. ${ }^{[660]}$ Diabetic neuropathy may cause structural and functional changes in the female genitalia and can disrupt the balance between receiving sexual stimuli and sexual response triggers. Hormonal imbalances in levels of estrogen and androgens can lead to FSD in women with diabetes. In a study from North India conducted in women with diabetes it was observed that $45.19 \%$ complained of desire disorder, $62.71 \%$ of arousal disorder, $84.75 \%$ of orgasmic disorder and $20.38 \%$ experienced pain disorder; the incidence of these disorder was higher in older women. ${ }^{[672]}$

\section{Considerations}

Gender, glycaemic control, comorbidities, lifestyle management and knowledge of sexual disorders and its management, cultural environment, psychological disorders and counselling should be considered when framing these recommendation for sexual dysfunction in patients with diabetes.

\section{Rationale and Evidence}

\section{Men}

- Longer duration of diabetes is considered a risk for ED. ${ }^{[664]}$

- Commonly associated comorbidities of diabetes including metabolic syndrome, obesity, hyperlipidemia, hypertension and autonomic neuropathy are also considered as risk factors of ED. ${ }^{[673]}$

- Anti-hypertensives, anti-depressants and fibrates are frequent concomitant medications consumed by diabetics and these are associated with increased risks of ED. ${ }^{[674]}$

- A significant association between ED and cardiovascular events, all-cause mortality, CHD and stroke has been reported in several studies. ${ }^{[675]}$ Meena et al. observed an increased cardiovascular risk in patients with T2DM and ED without overt cardiovascular disease (CVD) in comparison to patients without $\mathrm{ED}(34.87 \pm 18.82$ vs 20.91 $\pm 11.03 \mathrm{p}=0.002){ }^{[676]}$

- Below normal testosterone concentrations and higher rates of hypogonadism has been reported in men with diabetes as compared with the general population. ${ }^{[677]}$ Testosterone regulates the normal erectile functioning and evidence from studies suggests use of testosterone replacement in patients with diabetes and symptomatic hypogonadism. ${ }^{[678]}$ In a cross-sectional study conducted in India, the prevalence of hypogonadism wasfound to be $20.7 \%$ in T2DM patients. ${ }^{[679]}$
- Men with hypogonadism do not respond optimally to phosphodiesterase type (PDE)-5 inhibitors and in such patients testosterone replacement was observed to be effective in $50 \%$ patients. ${ }^{[678]}$

- Whether glycemic control has any effect on reduction of ED risk is unclear as studies have shown contrasting results. However, intensive lifestyle changes ameliorated worsening of ED and has shown an improvement in the overall International Index of Erectile Function (IIEF) score in overweight men with diabetes as compared with controls in the LOOK AHEAD study. ${ }^{[600]}$

- Men with T2DM and severe ED were found to have poor glycemic control, longer duration of untreated diabetes, later age of onset and poor quality of life..$^{[681]}$

\section{Women}

- Diabetes-induced neuropathy and vascular dysfunction may be mainly responsible for the FSD and the low Female Sexual Function Index may be associated with BMI. ${ }^{[670]}$

- Higher risk of FSD was observed in premenopausal as compared with postmenopausal women with diabetes.

- The risk factors associated with FSD include age, obesity, dyslipidemia, CVD, complications of diabetes, depression and marital status. ${ }^{[661,682]}$

- Based on the current evidence a clear correlation between FSD and CVD has not been established unlike in men with diabetes.

- In women, psychological and psychosocial factors contribute to FSD more than in men.

\section{IMPLEMENTATION}

- Normal sexual function is essential for the holistic well-being of an individual. Diabetes with its ever increasing prevalence is a cause of sexual dysfunction in both men and women. The vascular and neurological complications induced by diabetes constitute the underlying pathogenesis of these sexual dysfunctions. Association of diabetes with obesity, metabolic syndrome, hypertension, dyslipidemia and CVD are considered as risk factors for ED. The diagnosis of ED predicts further investigation of $\mathrm{CV}$ events in men with diabetes. Furthermore, symptoms of hypogonadism should be investigated by assessing the serum testosterone concentrations. A detailed history of FSD obtained in a compassionate and structured method is essential in women with diabetes. Although, limited evidence exists to show correlation between FSD and CV events, lifestyle modifications, glycemic control, care of genetic infections and resolution of psychosocial factors should be discussed and emphasized. 


\section{GLYCAEMIC Monitoring}

\section{ReCOMmEndations}

\section{Recommended Care}

- Monitor blood glucose control by measuring HbA1c using high-precision methods standardized and aligned to the international reference values

- Advise individuals with diabetes that maintaining an $\mathrm{HbA} 1 \mathrm{c}<7.0 \%$ minimizes the risk of developing complications.

- A lower HbA1c target may be considered if it is easily and safely achieved without hypoglycaemia

- A higher HbA1c target may be considered for individuals where previous attempts to optimize control were associated with unacceptable hypoglycaemia

- Treatment should be reviewed and modified if $\mathrm{HbAlc}$ level is above the agreed target on two consecutive occasions.

- Advice those in whom target HbA1c levels cannot be reached that any improvement is beneficial.

- SMBG enables patients to detect and prevent asymptomatic hypoglycaemia and glucose variability and to make appropriate adjustments in treatment medications and nutrition therapy to achieve HbAlc targets.

- In patients on insulin, a combination of HbA1c and SMBG is useful in achieving glycaemic control.

- Measure HbA1c every three to six months depending on level, stability of blood glucose control and changes in therapy and report HbA1c results in percentages.

- Anaemia must be excluded before a proper diagnosis based on HbAlc values is made. Anaemia and abnormal haemoglobin may affect the values obtained for $\mathrm{HbAl} \mathrm{c}$ in some assays. To determine whether abnormal haemoglobin is present, use high-performance liquid chromatography or mass spectrometry.

- Point-of-care capillary blood glucose meters should be used to measure blood glucose when patients are hospitalized. Blood glucose meters conforming to the latest ISO standards should be used.

- When prescribing CGM, robust diabetes education, training, and support are required for optimal continuous glucose monitor implementation and ongoing use.

\section{Limited Care}

- If $\mathrm{HbA} 1 \mathrm{c}$ measurement is not available, blood glucose should be measured either at point-of-care or in the laboratory.

- In very limited settings, diabetes control may need to be based on measurement of plasma glucose levels alone.

\section{BACKGROUND}

Monitoring blood glucose levels is critical to ensure optimum glycaemic control. It is a corner stone of diabetes care that may help physicians to adjust the treatment regime according to patient's need and help patients to follow the prescribed diabetes care. ${ }^{[683]}$ Glycated haemoglobin (HbA1c), which assesses the average level of blood glucose over 3 months, and self-monitoring of blood glucose (SMBG), which records the day-to-day blood glucose levels, are the two important tools for monitoring of glycaemic control. ${ }^{[684]}$ Measurement of HbAlc is known as a gold standard approach for monitoring diabetes in both research and in clinical settings ${ }^{[685-687]}$ Most guidelines recommend clinicians must perform $\mathrm{HbA} 1 \mathrm{c}$ measurements routinely in all patients with $\mathrm{T} 2 \mathrm{DM}$ as a part of continuing care ${ }^{[688,689]}$ Long-term hyperglycaemia as measured by HbA1C is associated with secondary macro-and micro-vascular complications due to diabetes. ${ }^{[690,691]}$ Therefore, patients with diabetes who do not reach appropriate glycaemic targets or are at an increased risk of developing complications require more intensive monitoring. Further, in Asian countries like India, religious aspects of weekly fasts, festivals, standard Indian diet rich in carbohydrates and reluctance to change the dietary habits, further support the need of regular glucose monitoring. Frequent SMBG, ${ }^{[685,692]}$ continuous glucose monitoring $(\mathrm{CGM})^{[693,694]}$ assessing impending glucose excursions (both hypoglycaemia and hyperglycaemia) and glycaemic variability ${ }^{[695]}$ are some of the methods of intensive glucose monitoring.

CGM fulfilled an unmet need in diabetes care by providing an option of automated glucose monitoring which may help improve glucose control in patients with uncontrolled T2DM and in patients on acute and intensive glucose lowering regimen. In special cases such as pregnant women, children and adolescents group, CGM may help monitor prandial insulin doses and other dietary decisions. ${ }^{[696]}$ The currentre commendations provide an insight on the importance and frequency of monitoring to be performed in order to facilitate medication and lifestyle changes when average $\mathrm{HbA} 1 \mathrm{c}$ values remain above targets levels. HbAlc level monitoring is a convenient and reliable alternative to plasma glucose tests to diagnose diabetes.

SMBG is an essential component of the modern diabetes treatment, it is the simplest and possibly most practical tool to assess efficacy and safety of glycaemic control. ${ }^{[697,698]}$ SMBG facilitates patients and healthcare providersto adjust their therapeutic regimen in response to blood glucose values and regulate the dietary intake, physical activity, and insulin doses to improve glycaemic control on a regular basis. ${ }^{[692,699]}$ Established advantages with SMBG include, achieving target $\mathrm{HbA} 1 \mathrm{c}$, reducing glucose variability and prediction of severe hypoglycaemia. ${ }^{[699]}$ SMBG complements HbA1c testing as it can differentiate the fasting, pre-prandial, and 
post-prandial hyperglycaemic levels, detect the glycaemic excursions, recognize and contribute in monitoring resolution of hypoglycaemia, and provide immediate feedback to patients about the effects of food choices, activity, and medication on glycaemic control. ${ }^{[700]}$

The International Diabetes Federation (IDF) and American Diabetes Association (ADA) recommend SMBG as an integral component of effective T2DM management. ${ }^{[4,690]}$ Despite substantial evidence of the benefits of SMBG, compliance to self-monitoring is reported "low" globally, ${ }^{[701]}$ particularly in developing countries like India, where patients usually seek treatment after complications have set in. This may be attributed to various factors such as lack of awareness, literacy levels, and the perception that SMBG is painful and costly. ${ }^{[702]}$

Target values for glucose control for HbA1c and capillary plasma glucose for prediabetes and diabetes as described by the IDF 2017 are as follows ${ }^{[703]}$ [Table 18].

\section{Recommendations for self-monitoring of blood glucose [Tables 19-22]}

Selecting a structured, flexible SMBG pattern that can be tailored to the clinical, educational, behavioural, and financial requirements of individuals with diabetes is recommended. ${ }^{[698]}$ As it is important to determine the frequency and intensity of SMBG needed to support the chosen treatment regimen, one should also consider practical obstacles to monitoring, such as affordability or access and individualize glycaemic target and modify monitoring patterns accordingly. ${ }^{[704,705]}$

- In patients with pre-existing diabetes or GDM, target blood glucose levels should be 70 to $90 \mathrm{mg} / \mathrm{dL}$ fasting, $<140 \mathrm{mg} / \mathrm{dL}$ 1-h post-prandial, and $<120 \mathrm{mg} / \mathrm{dL} 2$-h postprandial.

- Individuals with insulin-treated diabetes should be advised to perform SMBG on a daily basis, failing which, at least weekly monitoring should be encouraged. Ideal SMBG: seven tests/day i. e. three before and three after each meal and one test at 3 am. ${ }^{[684]}$

- Pregnant women on lifestyle modifications should have a day profile once a week. This should include one fasting and three post-prandial values at least once a week or staggered over a week. ${ }^{[684]}$

Table 18: Target values for glucose control for glycosylated haemoglobin

\begin{tabular}{lcccc}
\hline & Normal & Prediabetes (IGT) & T2DM & Target \\
\hline HbA1c $(\%)$ & $<5.7$ & $5.7-6.4$ & $>7.0$ & $<7.0$ \\
FPG $(\mathrm{mg} / \mathrm{dL})$ & $<110$ & $\geq 110$ and $<126$ & $\geq 126$ & 115 \\
PPG $(\mathrm{mg} / \mathrm{dL})$ & $<140$ & $\geq 140$ and $<200$ & $\geq 200$ & 160 \\
\hline
\end{tabular}

DM: Diabetes mellitus, FPG: Fasting plasma glucose,

HbA1c: Glycosylated haemoglobin, IGT: Impaired glucose tolerance, PPG: Postprandial glucose, T2DM: Type 2 DM
- In elderly patients, the frequency of SMBG should be once daily (different time each day) in the initiation phase and later it should be reduced further to two to three times per week.

\section{Considerations}

The decisions on clinical monitoring of glycaemic levels in T2DM patients was based on the local factors such as availability of newer technologies and cost of monitoring that were reviewed in Indian context [Table 23].

\section{Rationale and Evidence}

\section{Glycosylated haemoglobin for monitoring blood glucose}

- Regular monitoring of HbAlc will facilitate identification of patients with poor glycaemic control and help both physicians and patients to take necessary steps to achieve desired glycaemic targets. ${ }^{[708,709]}$ Though frequent monitoring of $\mathrm{HbAlc}$ is associated with reduced diabetes-related complications and improved metabolic control, ${ }^{[295,709]}$ most patients do not understand or are unaware of the importance of glycaemic monitoring. Therefore, educating patients and improving understanding on HbAlc levels for optimal glycaemic control is vital. ${ }^{[709,710]}$

- The concept of estimated average glucose (eAG) was introduced following introduction of continuous ambulatory blood glucose monitoring. ${ }^{[711]}$ The eAG may help people with diabetes, relate their $\mathrm{HbAlc}$ to daily glucose monitoring and highlight any inaccuracies in $\mathrm{HbA} 1 \mathrm{c}$ measurement relative to glucose levels. ${ }^{[712]}$ Calculators are available for converting $\mathrm{HbA} 1 \mathrm{c}$ to eAG in both $\mathrm{mmol} / \mathrm{L}$ and $\mathrm{mg} / \mathrm{dL}$. Measurement of glucose levels, before meals, after meals and fasted state are often recommended as a substitute for $\mathrm{HbA} 1 \mathrm{c}$ when the latter is either unavailable or inappropriate.

- Abnormal haemoglobin levels are known to affect HbAlc values in a way that can significantly alter the results with regard to diabetes control..$^{713]}$ Therefore, it is important to consider haematological factors that can confound HbA1c levels in people with diabetes; best detected using HPLC-based assays.

- Anaemia significantly impacts HbAlc levels. In a cross-sectional study, the mean HbAlc in patients with controlled diabetes with iron deficiency anaemia (IDA) was significantly higher than those without IDA $(7.86 \pm 0.11 \%$ vs $5.45 \pm 0.038 \%[\mathrm{p}<0.05])$ and the HbAlc values were inversely proportional to total haemoglobin $(\mathrm{p}<0.05){ }^{[714]}$

- Further, significantly higher HbAlc levels are observed in patients with IDA than healthy individuals $(5.51 \pm 0.696 \mathrm{v} / \mathrm{s} 4.85 \pm 0.461 \%, \mathrm{p}<0.001)$ and the $\mathrm{HbA} 1 \mathrm{c}$ levels significantly decline following iron supplementation $(\mathrm{p}<0.001){ }^{[35]}$ Therefore, HbA1c results in diabetes patients 
Table 19: Recommended care for frequency/timing of SMBG[698]

\begin{tabular}{|c|c|c|c|}
\hline \multicolumn{2}{|c|}{ T2DM on OADs } & \multicolumn{2}{|c|}{ T2DM on insulin or insulin + OADs } \\
\hline $\begin{array}{l}\text { New onset/uncontrolled/ } \\
\text { during acute illness }\end{array}$ & Stable/well-controlled & $\begin{array}{l}\text { New onset/uncontrolled/ } \\
\text { during acute illness }\end{array}$ & Stable/well-controlled \\
\hline $\begin{array}{l}\text { Patients on SU or meglitinides: } \\
\text { At least } 4 \text { times/day and } \\
\text { should include preprandial and } \\
\text { bedtime levels. } \\
\text { Patients on other OADs: } \\
\text { At least FBG on alternate days }\end{array}$ & $\begin{array}{l}\text { At least } 4 \text { tests in a week on } 4 \\
\text { consecutive days or on alternate } \\
\text { days (including an FBG and } 3 \\
\text { postprandial values) }\end{array}$ & $\begin{array}{l}\text { At least } 4 \text { times/day and } \\
\text { shouldinclude preprandial } \\
\text { and bedtimelevels } \\
\text { Must check whenever } \\
\text { hypoglycaemia is suspected }\end{array}$ & $\begin{array}{l}\text { Paired testing at least 3-4 days in a week ( } 1 \text { day/ } \\
\text { week pre- and post-breakfast, } 1 \text { day/week pre- and } \\
\text { post-lunch, and } 1 \text { day/week pre- and post-dinner) } \\
\text { or as frequently as possible } \\
\text { Must check whenever hypoglycaemia is suspected }\end{array}$ \\
\hline
\end{tabular}

Table 20: Recommended care for frequency/timing of self-monitoring of blood glucose for diabetes in pregnancy $^{[684]}$

\begin{tabular}{ll}
\hline Patients on lifestyle modifications & Patients on OADs or insulin \\
\hline $\begin{array}{l}\text { A day profile once a week-FBG and } \\
3 \text { postprandial values at least once a } \\
\text { week or staggered over the week }\end{array}$ & $\begin{array}{l}\text { At least } 4 \text { times/day (FBG } \\
\text { and } 3 \text { postprandial values) }\end{array}$ \\
\hline
\end{tabular}

FBG: Fasting plasma glucose, OADs: Oral antidiabetics

with IDA should be interpreted carefully. IDA has to be corrected before a proper diagnosis is made.

- Measurement of blood glucose using blood glucose meters on admission to hospital wards helps to identify patients with hypoglycaemia or hyperglycaemia. Considering that in developing nations like India, where cost is major barrier for monitoring, these devices should be accurate, cost effective and field testing specifically tailored for Asian and Indian needs is imperative. ${ }^{[715]}$

- A study that assessed knowledge and attitude towards self-monitoring and the impact of SMBG on glycaemic control revealed that patients who monitored $\geq 3$ times had significantly better glycaemic control of HbA1c (7.1-8\%) than those who monitored $<3$ times $(\mathrm{p}=0.021) .{ }^{[716]}$ Insulin self-titration interventions based on structured SMBG are associated with significant reduction in $\mathrm{HbA} 1 \mathrm{c}$ during a follow-up of 12 weeks with a trend towards greater effectiveness in improving glycaemic control than conventional treatment, with no increase in incidence of hypoglycaemia or body weight gain. ${ }^{[717]}$ Comparative studies in patients with T2DM on insulin across cohorts of regular SMBG users versus SMBG non-users have demonstrated that $\mathrm{HbAlc}$ levels in regular SMBG users were lower by $0.7-1.1 \%{ }^{[718-720]}$

\section{Glucose measurement}

- Plasma glucose is the most preferred measure in most modern laboratories. Readings based on whole blood measurements are lower, due to the volume occupied by haemoglobin. Capillary blood glucose strips measure the glucose in the plasma of the capillary blood sample, but may be calibrated to give results either as plasma or sometimes whole blood glucose (check meter instructions).

\section{Continuous glucose monitoring}

- Garg et al. have demonstrated an improvement on glycaemic excursion in insulin-treated T2DM patients using Real time-CGM, showing a significant reduction of the time spent in hypo- and hyper-glycaemic range with an increased time spent in the target glucose range as well as a significant reduction of nocturnal hypoglycaemia in the Real time-CGM group. ${ }^{[721]}$

- Mohan et al. evaluating the use of retrospective CGM concluded that it can effectively help healthcare professionals with insights for initiating changes to treatment regimens, diet and exercise behaviours and provided patients with improved knowledge of the importance of therapy compliance by demonstrable reductions in $\mathrm{HbAlc} .^{[722]}$

- A retrospective analysis based on a blinded study of glycaemic control in 296 T2DM adults using masked professional CGM (P-CGM) revealed that the predominant pattern of hyperglycaemia was postprandial while previously unknown hypoglycaemia was found in $38 \%$ of the patients; over half of the cases were nocturnal. The mean HbAlc of the P-CGM group significantly dropped at 6 months from baseline $(\mathrm{P}<0.0001)$. The frequency of performing SMBG was also found to be significantly increased. P-CGM motivated the patients for diabetes self-care practices, resulting in an improvement in glycaemic control over a wide range of baseline therapies. ${ }^{[336]}$

\section{IMPLEMENTATION}

There should be access to a laboratory or site-of-care test monitored by certified quality assurance schemes for measurement of $\mathrm{HbAlc}$. In instances where $\mathrm{HbAlc}$ measurement is inappropriate, such individuals must be identified by careful review of haematological parameters and other factors that can affect HbA1c values. Provision of capillary blood glucose meters and strips need to be assured in hospitals and clinics. It is important to ascertain whether there are contraindications for use of a particular type of glucose meter in a particular patient. It is essential to establish whether glucose meters report values for plasma or blood and to ensure that schemes for monitoring the quality of their output are in place. Blood glucose meters should be calibrated on regular basis and their use in hospitals should be restricted to trained personnel. 
Table 21: Recommendations for glycaemic targets in the elderly ${ }^{[06,707]}$

\begin{tabular}{lccc}
\hline Target glycaemic levels & Healthy elderly & Elderly with intermediate health status & Elderly with poor health status \\
\hline HbA1c (\%) & $<7.5$ & $<8.0$ & $<8.0$ \\
Fasting or preprandial glucose (mg/dL) & $90-130$ & $90-150$ & $100-180$ \\
Bedtime glucose (mg/dL) & $90-150$ & $100-180$ & $110-200$
\end{tabular}

HbA1c: Glycosylated haemoglobin

\section{Table 22: Limited care for frequency/timing of self-monitoring of blood glucose}

\begin{tabular}{|c|c|c|}
\hline & New onset/uncontrolled/DM during acute illness & Stable/well-controlled \\
\hline T2DM on OADs & $\begin{array}{l}\text { Patients on SU or meglitinides: At least FBG alternate days } \\
\text { Patients on other: At least FBG once a week }\end{array}$ & $\begin{array}{l}\text { At least } 4 \text { tests in a month-at least } 1 \text { test/week (including } \\
\text { a FBG and } 3 \text { postprandial values in a month) }\end{array}$ \\
\hline $\begin{array}{l}\text { T2DM on insulin or } \\
\text { insulin + OADs }\end{array}$ & $\begin{array}{l}\text { At least FBG and one more preprandial value every day } \\
\text { Must check whenever hypoglycaemia is suspected }\end{array}$ & $\begin{array}{l}\text { At least one value on alternate days at different times of } \\
\text { the day, with at least one FBG every week } \\
\text { Must check whenever hypoglycaemia is suspected }\end{array}$ \\
\hline & Patients on lifestyle modifications & Patients on OADs or insulin \\
\hline Diabetes in pregnancy & $\begin{array}{l}\text { One FBG and one postprandial value every week (any } \\
\text { meal, preferably largest meal of the day) }\end{array}$ & $\begin{array}{l}\text { Paired testing every day (pre- and post-breakfast on } 1^{\text {st }} \\
\text { day, pre- and post-lunch on } 2^{\text {nd }} \text { day, pre- and post-dinner } \\
\text { on } 3^{\text {rd }} \text { day, and then keep repeating the cycle) }\end{array}$ \\
\hline
\end{tabular}

DM: Diabetes mellitus, FBG: Fasting blood glucose, OADs: Oral antidiabetics, SU: Sulphonylureas, T2DM: Type 2 DM

\section{Table 23: Other methods of clinical monitoring}

\begin{tabular}{ll}
\hline Type of monitoring & Recommended care \\
\hline $\begin{array}{l}\text { Complete history and } \\
\text { physical examination }\end{array}$ & $\begin{array}{l}\text { A complete history and physical examination is recommended } \\
\text { Periodicity: Annually } \\
\text { Ophthalmic }\end{array}$ \\
& $\begin{array}{l}\text { Detailed exam by qualified ophthalmologist } \\
\text { Periodicity: At diagnosis and every 2 years if there is no retinopathy }\end{array}$ \\
Smoking cessation & $\begin{array}{l}\text { Counselling by physician at every visit } \\
\text { BP measurement }\end{array}$ \\
Beasurement of lipids & At diagnosis or at age 40 years and periodically (6 monthly) thereafter \\
Screening for CVD & A resting ECG may provide useful information on baseline cardiac status \\
& and for future reference \\
& 2d ECHO when required \\
Microalbuminuria & At diagnosis and annually thereafter
\end{tabular}

Limited care
As for recommended care
Patients to be referred to ophthalmologists if
retinopathy is suspected

As for recommended care

As for recommended care

At diagnosis or at age 40 years at least

As for recommended care

If resources are limited and technical issues may consider use of ACEI/ARB if BP is $>140 / 80$

Dipsticks for MA can be used

Every patient's urine should be examined routinely and microscopically?

As recommended by IDF

Additional training required

Test for vibration with $128 \mathrm{hz}$ tuning fork or a $10 \mathrm{~g}$ monofilament, pinprick sensation ankle jerk

neuropathy

At diagnosis

As for recommended care

History of claudication, distal pulses and ABI

Additional training required

disease

At diagnosis and annually

Comprehensive foot

care

Assessment of foot pulses, and testing for loss of protective sensation

As for recommended care

Additional training required

(10 g monofilament plus testing any one of: Vibration using $128 \mathrm{~Hz}$ tuning

fork, pinprick sensation, ankle reflexes, or vibration perception threshold)

BP: Blood pressure, CV: Cardiovascular, CVD: CV disease, ECG: Electrocardiogram, ABI: Ankle-brachial index, ACEI: Angiotensin converting enzyme, ARB: Angiotensin receptor blocker, IDF: International Diabetes Federation 


\section{TECHNOLOGIES}

\section{RECOMMENDATIONS}

\section{Recommended Care}

Continuous glucose monitoring (CGM)

- CGM (includes AGP) should be considered in conjunction with SMBG and HbA1C for glycaemic status assessment in those T2DM individuals treated with intensive insulin therapy who are not achieving glucose targets

- CGM may be considered in women with GDM or pregnant women with T2DM and as a supplemental tool to SMBG in individuals with hypoglycaemia unawareness and/or frequent hypoglycaemic episodes.

- CGMs should preferably be used daily to gain maximal benefit.

- CGMs can be a helpful tool in diabetes education by facilitating effective communication between clinicians and patients. All users should get trained on how to interpret and respond to their glucose data.

- Only CGM systems with an acceptable level of sensor accuracy should be used and when assessing hypoglycaemia the accuracy of the CGM data in the lower glycaemic range should be considered.

Continuous subcutaneous insulin infusion (CSII) or insulin pump therapy

- CSII or insulin pump therapy may be considered in paediatric patients or in adults on $\geq 4$ insulin injections per day (intensively managed insulin-dependent T2DM).

Common indications being:

- High HbA1C levels on injection therapy

- Recurrent episodes of hypoglycaemia or hypoglycaemia unawareness

- Patients on high doses of insulin or poor glycaemic control despite intensive therapy

- Presence of or a future risk of diabetes-related complications, or recurrent DKA/recurrent hospitalizations

- Dawn phenomenon

- Glycaemic variability causing challenges in diabetes management

- Unpredictable food or meal intake patterns

- Patients seeking improved quality of life

- Insulin pump therapy seems to be safe and effective for maintaining glycaemic control and for better outcomes in pregnancies complicated by GDM/ T2DM and requiring large insulin doses. However, it is not recommended as a part of routine practice.

- During hospital admissions, CSII is not recommended in critically ill patients if the hospital/ICU staff is not familiar with the device

- In non-critically ill patients, continued use of CSII is recommended if the patient can manage the use of the device himself or has trained assistance for the same.

- CSII should be prescribed to only those eligible patients who are willing and motivated to monitor glucose levels at least four times a day, quantify food intake, and comply with follow-up. Patients must be psychologically stable and in the case of young candidates, they should have adequate support from motivated caregivers who can learn and can commit to the different aspects of diabetes management.

- CSII should only be initiated at a well-equipped centre that has trained resources to initiate and follow-up the patients on CSII.

- Continuous training and retraining would be required to learn the techniques and excel in CSII management.

Clinical decision support tools and diabetes management platforms

- Technologies that aid patients and/or healthcare providers in the diagnosis and management of diabetes, can improve both the short-term and long-term disease outcomes.

- Adequate training need to be provided to the healthcare professionals in using the clinical decision support tools and diabetes management platforms.

- From among the various diabetes self-management tools and platforms available, patients must be encouraged to adopt the most appropriate tool that would best suit their disease needs and lifestyle.

- Patients must be encouraged to seek timely guidance and frequent reassessment from a trained healthcare team and must be made aware that the adoption of various diabetes self-management tools does not diminish the importance of the former.

\begin{tabular}{|lll|}
\hline \multicolumn{2}{|l|}{ Recommendations on the technologies suggested for recommended care and limited care } \\
\hline Technology & Recommended Care & Limited Care \\
\hline Glucometer & Yes & Yes \\
\hline Diabetes Apps & Yes & Yes \\
\hline Insulin pump & $\begin{array}{l}\text { Yes; indications should be discussed and apart } \\
\text { from usual indications, CSII as an option to } \\
\text { improve quality of life of the individual should } \\
\text { be discussed. }\end{array}$ & $\begin{array}{l}\text { Campelling indication } \\
\text { comere is a }\end{array}$ \\
\hline CGM & Yes; indications should be discussed & $\begin{array}{l}\text { Can be discussed when there is a } \\
\text { compelling indication }\end{array}$ \\
\hline
\end{tabular}




\section{BACKGROUND}

Technologies have gradually become indispensable in the management of diabetes. Technologies such as insulin pump, artificial pancreas, CGM are relatively expensive. However, connected glucose meters, telemedicine and mobile apps for enhancing adherence to therapies and to enable coaching could be cheaper options even for limited care in the absence of which it is difficult to reach the goals of management. There have been a few guidelines available both internationally as well as specific to the Indian scenario, regarding the use of various diabetes technologies. ${ }^{[698,723-}$ ${ }^{732]}$ The current RSSDI guidelines are aimed at preventive technologies which are directed at saving the cost and future complications. Most of the technologies discussed are those which can be used by any modern medicine physician with appropriate training.

\section{Rationale and Evidence \\ Blood glucose meters}

SMBG with a quality glucose meter has been proven to be useful at any stage of diabetes provided a structured SMBG protocol is implemented with patient centred approach. Glucose monitoring, particularly SMBG is considered as an integral part of diabetes care ${ }^{[698,726,733,734]}$ since achieving optimal glycaemic control has been proven to be associated with reductions in both macro-and microvascular complications of the disease. ${ }^{[169,295,735]}$ SMBG has been demonstrated to be helpful or to correlate with effective management in both insulin-treated and noninsulin-treated diabetes. ${ }^{[295,333,334,726,736-738]}$ Many different models of glucometers are available to suit the needs of the patients and differ in terms of their accuracy, amount of blood needed for each test, ease of use, pain associated with using it, testing speed, overall size, memory functions to store the test results, likelihood of interferences, ability of transferring data, procurement costs of the meter and accessories, special features such as automatic timing, error codes, large display screen etc. ${ }^{[739]}$ Regarding the accuracy of the glucometers, though there are several current standards, the most commonly followed are those of the International Organization for Standardization (ISO 15197:2013) ${ }^{[740]}$ and the U. S. Food and Drug Administration (USFDA). ${ }^{[71-743]}$ The marketing strategies in India are highly influenced by the cost of meter and strips and quite often the quality is compromised. The CONTOUR ${ }^{\circledR}$ PLUS ONE smart meter The OneTouch Verio Flex ${ }^{\circledR}$ meter, Accu Chek Instant etc. are some among the globally popular glucose meters available in India. Currently connected glucose meters provide a patient friendly visualisation of blood glucose trends, time spent in range, time spent in hypoglycaemia, cloud storage, ability to email the digital blood glucose diary to the physician's office along with storing the entire information. These glucose meters also provide options for users to enter data on insulin and other medications, calculate insulin carb ratio, insulin correction factor etc. thereby providing a comprehensive digital solution to a motivated patient.

\section{Continuous glucose monitoring systems}

$\mathrm{HbA1C}$, the traditional metric for assessing glycaemic control in prognosis of diabetes, fails to capture intraand interday glycaemic excursions which have been identified as risk factors for micro-and macrovascular complications. Therefore frequent glucose monitoring should be performed to reveal the underlying glycaemic variability in order to complement HbA1C..$^{[74,745]}$ A structured SMBG cannot predict impending hypoglycaemia or alert for hypoglycaemia. ${ }^{[746,747]}$ Thus SMBG and HbA1C monitoring though an integral component of diabetes management, CGM is now considered as a complementary method for the assessment of glycaemic control. ${ }^{[743,748,749]}$ CGM devices are comprised of a transcutaneous probe (sensor) that procures interstitial fluid glucose readings every 5-15 minutes depending on the make. CGM sensors can stay in place from 6 to 14 days or even 6 months at a time, depending on the model used. They fetch information regarding hourly, daily, and weekly glucose trends and patterns and thus offer opportunities for detailed analyses of the patient data. A variety of metrics have been proposed including average glucose, percentage of time in hypoglycaemic, hyperglycaemic and target ranges. This has been further endorsed by the fact that $\mathrm{CE}$ (Conformite Europeenne) and US FDA have approved some CGM devices for adjustment of insulin dosages [Figure 15]. ${ }^{[750-752]}$ CGMs broadly fall into two categories. The 'Professional CGM' (P-CGM), meant for the healthcare professionals to record the patient's ambulatory glucose values, which are then downloaded and used for retrospective review and analysis. The 'Personal CGMs' are patient-owned devices where the sensor glucose values are sent wirelessly to a separate receiver device where they can be viewed in real-time (RT-CGM).

\section{Continuous subcutaneous insulin infusion}

CSII or insulin pump therapy [Figure 16] is an alternative insulin delivery method and is superior in many aspects to ordinary syringes and insulin pens. An insulin pump is a small computerised device that can be worn externally, delivers a continuous infusion of rapid-acting insulin and attempts to mimic the function of a normal pancreas. It delivers insulin in two ways: a continuous, small dose of insulin to maintain glucose levels stable between meals and overnight (basal rate); and a much higher rate of insulin taken before meals to "cover" the food one plans to eat (bolus rate).

In India, insulin pumps were introduced in 2004. No definite reimbursement policies exist in the country and hence this lifesaving technology is still not available to the most deserving candidates except on charity. ${ }^{[760]}$ Five different types of insulin pump models currently available in India include Medtronic's (MiniMed, Inc., Northridge, CA) Minimed 715, Minimed 722, Minimed 620G and Minimed 640G). The MiniMed 


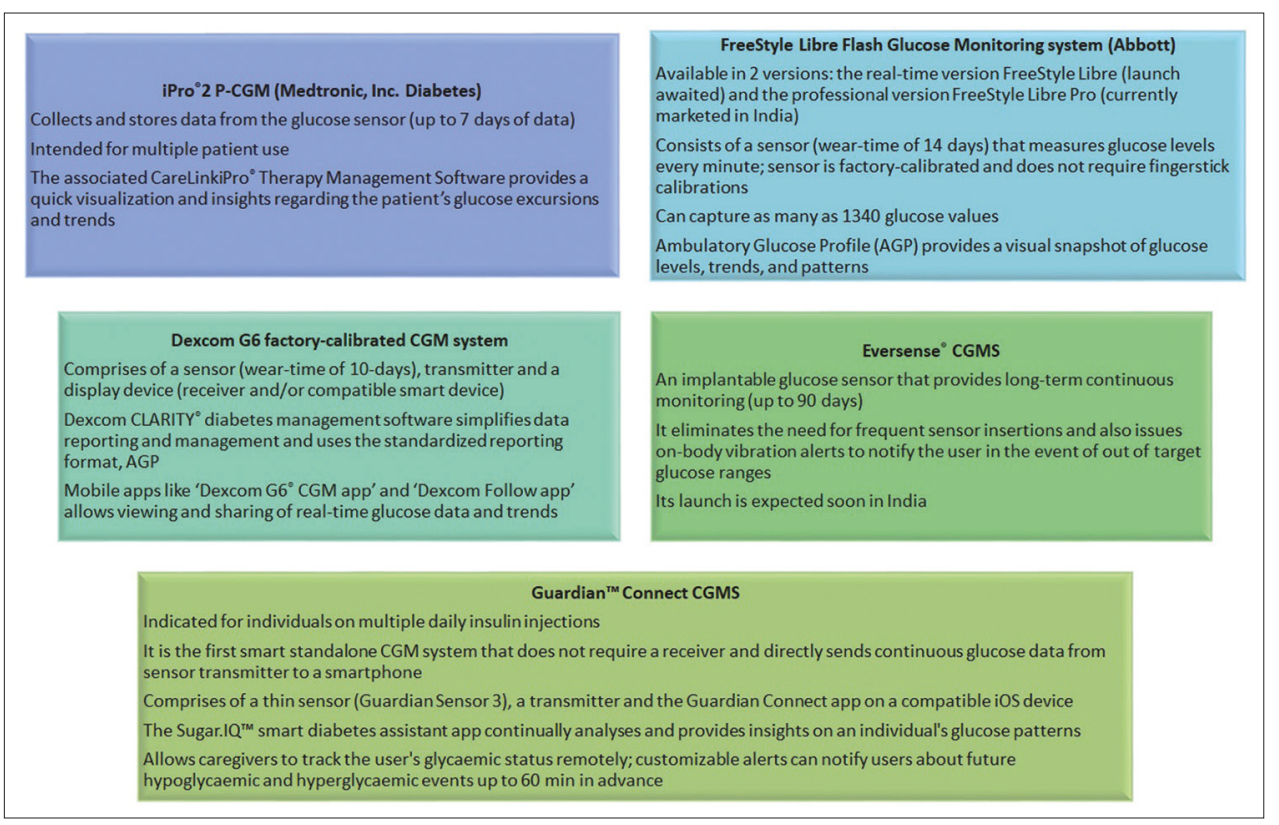

Figure 15: Currently available continuous glucose monitoring models ${ }^{[336,744,753-757]}$

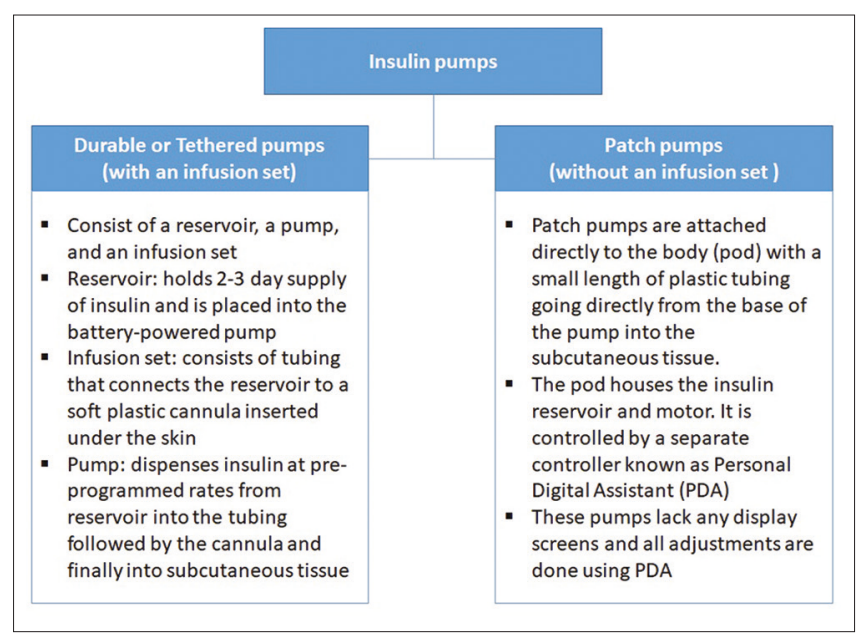

Figure 16: Types of insulin pumps[758-760]

640G is the first-generation artificial pancreas available in India since 2015 indicated for both T1DM and T2DM. It has built-in intelligent features for increased protection including the SmartGuard ${ }^{\mathrm{TM}}$ technology, active insulin tracking, bolus progress bar, and predictive battery life. The SmartGuard technology predicts hypoglycemic events up to 30 minutes in advance and automatically stop insulin delivery (Suspend before low option), and automatically resume insulin delivery when the glucose levels recover. Multiple low limits can be set throughout the day to give increased protection in times of need. A completely Closed Loop system enable complete automation of insulin delivery with minimal patient interaction. ${ }^{[761-763]}$ The latest development, MiniMed ${ }^{\text {TM }}$ 670G insulin pump, is based on Hybrid Closed Loop Technology and has an auto mode feature that automatically adjusts basal insulin delivery every 5 min based on sensor glucose to maintain blood glucose levels as close to a specific target as possible. Patients receive prandial insulin by entering carbohydrate amount into the bolus calculator. The device is expected to be available in India in the near future. ${ }^{[764]}$ The Micro Solo Patch Pump, Medtrum insulin pump with sensor, etc. are also expected to be soon available in India. ${ }^{[765-767]}$

Even though CSII being the most physiological mode of insulin delivery and has several advantages over conventional insulin delivery modes, ${ }^{[768-771]}$ it is not meant for everyone. The diabetes care provider and the patient need to make choices based on the pros and cons of the treatment option. . $^{[722-779]}$

\section{Technologies to improve clinical decision support and treatment compliance}

The overall quality of diabetes care still remains suboptimal due to various patient and provider related factors. An organized, systematic approach in diabetes self-management and a continued support from a trained multidisciplinary diabetes care team are thus highly crucial for achieving optimal outcomes. ${ }^{[780,781]}$ Many solutions have been identified or are being employed to achieve this such as redesigning the organization of the care process, empowering and educating patients, implementing clinical decision support systems such as electronic health record tools, using diabetes detection or management apps and platforms for patients and healthcare professionals etc. ${ }^{[780,782,783]}$

Clinical decision support systems (CDSS) are applications that can analyse data and aid healthcare providers to make clinical decisions and improve patient care. Classic CDSSs may include various features like alerts, reminders, order 
sets, drug-dose calculators etc. that automatically remind the doctors of a specific action, or care summary dashboards that provide performance feedback on quality indicators thereby ensuring patient safety and improved health outcomes. Both commercially and locally developed CDSSs have been found to be effective at improving healthcare process measures across diverse settings..$^{[784,785]}$

Telemedicine is a disease management strategy whose basic concept matches with that of a CDSS. With the aid of telecommunications, telemedicine facilitates remote delivery of health-related services and clinical information. ${ }^{[786]}$ Various telemedicine modalities have been proven to be effective and safe across various patient populations irrespective of their type of diabetes. They have been also associated with time savings, cost savings, high appointment adherence rates, and high patient satisfaction. In populations with limited access to care, telemedicine effectively improves the overall disease outcomes. ${ }^{[787-791]}$ Diabetes Tele Management System, DTMS ${ }^{\circledR}$, a telemedicine-based diabetes care implemented at a diabetes care hospital in South India since the 1990s, is now well proven and accepted as a simple and cost-effective tool for comprehensive diabetes management. ${ }^{[791-793]}$

\section{IMPLEMENTATION}

Almost ninety percent of the world's population is estimated to be within the reach of a mobile network ${ }^{[794]}$ and the number of smartphone users also seems to be on the rise. Therefore, the feasibility of mobile apps to empower patients and healthcare providers is now a step higher than other approaches. Numerous mobile apps for diabetes management are nowadays available to help clinicians and/or the patients themselves to track and manage diet, physical activity, blood glucose target and medications. Examples include Life in Control, Medios Technologies AI assistant to detect diabetic retinopathy, mySugr etc. ${ }^{[95,795-797]}$ Diabetes technologies are meant for saving the time and better short term and long term outcomes. Choice should depend on the knowledge, infrastructure and the need.

\section{Special Situations}

\section{Type 2 Diabetes Mellitus and Surgery}

\section{ReCOMMENDATIONS}

\section{Recommended Care}

- Conduct preoperative assessments: baseline history of diabetes, symptoms of microvascular and macrovascular complications, physical examination HbA1c, blood glucose level, serum electrolytes and creatinine level, and current treatment regimen

- Maintain post prandial glucose of 140-180 mg/dL in-hospital ICU and 100-180 mg/dL as a guideline for general care medical and surgical wards

- Anti-diabetic agents like sulfonylureas, meglitinides, TZDs, SGLT-2 inhibitors, GLP-1 agonists must be discontinued on the day of surgery and metformin should be discontinued a night before surgery.

- In patients undergoing surgery, insulin basal-bolus regimen should be preferred

- For longer and complex surgeries IV insulin infusion is recommended

- Monitor blood glucose more frequently ranging from $0.5-2 \mathrm{~h}$

- On the day of surgery, avoid alterations in long acting basal insulin unless there is a tendency of hypoglycaemia or if patient is on diet restriction preoperatively. $75-100 \%$ of long acting insulin daily dose may be used on the day of surgery

- Patient should be provided with clear instructions about the return to their preoperative OADs and management of hypoglycaemia

- Resume the regular OAD medications only after the patient is medically stable and retaining oral meals regularly. Do not resume metformin in patient with renal dysfunction.

- Non-emergency procedures should be cancelled if patients have metabolic abnormalities (DKA, HHS, etc.) or glucose level $>400-500 \mathrm{mg} / \mathrm{dL}$

- Multidisciplinary care team within an institution should formulate appropriate protocol to be followed during the hospital/surgical course for hyperglycaemia screening, monitoring, and treatment to reduce errors and improve postoperative outcomes.

\section{Limited Care}

- Delay surgery until fluid volume status (BUN, creatinine and urine output) are stable and metabolic (pH, plasma glucose, creatinine, BUN, electrolytes) control is achieved

- Tailor the post prandial insulin requirements according to the nutritional mode of patient

- Avoid respective doses of subcutaneous insulin to prevent "stacking" of insulin 


\section{BACKGROUND}

Patients with diabetes experience higher number of hospitalisation and surgeries with longer hospital stays, higher treatment costs and greater risks of morbidity and mortality than non-diabetics. ${ }^{[798-800]}$ Surgeries in patients with diabetes can be categorized as major or minor. Major inpatient surgeries are defined as procedures requiring general, epidural, or spinal anaesthesia for $\geq 1 \mathrm{~h}$ as well as hospitalization for $>1$ day while all other outpatient procedures may be defines as minor surgeries. ${ }^{[801,802]}$

Surgical procedures may result in a number of metabolic perturbations that can alter normal glucose homeostasis. Persistent hyperglycaemia prior to and during surgical procedures may lead to postoperative complications like cerebral ischemia, endothelial dysfunction, postoperative sepsis, acute renal failure and surgical site infection (most common complication) and may also impair wound healing in patients with diabetes. ${ }^{[803,804]}$ Surgical stress may lead to hyperglycaemic hyperosmolar syndrome (HHS), the most common postoperative complication associated with $42 \%$ mortality rate along with diabetic ketoacidosis (DKA) during or after surgery. ${ }^{[802,803,805]}$ Furthermore, increased stress leads to increase in the counter regulatory hormones causing insulin resistance and the resulting hyperglycaemia impairs neutrophil function and trigger overproduction of inflammatory cytokines and reactive oxygen species which causes vascular and immune dysfunction, and cellular damage. ${ }^{[804]}$ Therefore, to minimize these negative consequences and improve the postoperative outcomes it is important to carefully manage the glycaemic level in diabetic patients undergoing major surgeries including orthopaedic and cardiac. ${ }^{[805]}$ The treatment recommendations for patients with T2DM should be individualized-based on the severity of diabetes, usual their standard diabetes regimen, level of glycemic control, types of surgical procedures (major/minor) ${ }^{\left[{ }^{[82]}\right.}$ Overall, the management goal in diabetic patients undergoing surgery should be optimization of metabolic control, adequate fluid repletion and postoperative care management with or without insulin to improve surgical outcomes. ${ }^{[798,804,805]}$

\section{Preoperative assessment}

Early risk assessments can minimize the incidence of perioperative and postoperative morbidities and reduce mortality rates as it provides an opportunity for planned intervention, proper arrangement and long-term follow-up. ${ }^{[799]}$ Physicians and multidisciplinary care teams must comprehend strategic plan to optimize glycaemia management in diabetic patients undergoing surgery. ${ }^{[806]}$

\section{Perioperative glycaemic targets and assessment}

Preoperatively, the ADA recommends a perioperative glucose target of 80 to $180 \mathrm{mg} / \mathrm{dL}$ as reasonable blood glucose maintenance. It is mandatory that the preoperative evaluation for surgical procedures must be conducted and must include assessment of glycaemic control and presence of any diabetesrelated complications. Measurement of serum creatinine level to assess DKD, haemoglobin $\mathrm{HbA1c}$, and blood glucose level are the critical baseline laboratory data that must be assessed ${ }^{[806]}$ Other critical assessments that must be considered are enumerated below [Table 24].

\section{Preoperative management}

\section{Patients treated with oral medications and/or noninsulin} injectable

Metformin should be discontinued a day before surgery due to the high risk of lactic acidosis with mortality rate of approx. $50 \% .{ }^{[807]}$ OADs mainly sulfonylureas and meglitinides, in fasting state have potential to cause hypoglycaemia and trigger endogenous secretion of insulin, independent of the glucose level, hence should be discontinued 1 day before surgery. Further, sulfonylureas and meglitinides increase the risk of myocardial ischemic injury and may be associated with increased risk of cardiovascular events and mortality. ${ }^{[808]}$ SGLT-2 inhibitors are associated with high risks for DKA and volume depletion. There have been a number of case reports of euglycemic ketoacidosis in the perioperative setting and hence SGLT-2 inhibitors should be stopped a day prior to surgery. ${ }^{[809-811]}$ DPP-4 inhibitors may be discontinued before surgery; however, in a recent study establishing the safety and efficacy of sitagliptin alone or sitagliptin in combination with basal insulin in hospitalized medical and surgical patients demonstrated good tolerability and low risk of hypoglycaemia and can be considered as a viable option in the perioperative setting. ${ }^{[006,812]}$ Due to slow gastric motility GLP-1 agonists (exenatide, liraglutide) are usually withheld the day before surgery. ${ }^{[809,813]}$ However, in cardiac and noncardiac surgical patients addition of a GLP-1 agonists to insulin therapy has demonstrated improved perioperative glycaemic control. ${ }^{[814,815]}$ AGIs (acarbose, miglitol) lower the absorption of glucose after meals, but these agents do not have any effect in the preoperative fasting states, and hence should be discontinued until the patient resumes eating. ${ }^{[816]}$ TZDs should be avoided due to risks like congestive heart failure, fluid retention and peripheral oedema. ${ }^{[812]}$

\section{Patients treated with insulin}

Insulin being the most preferred choice of drug for patients undergoing surgery the basal-bolus regimen is the best protocol as it is associated with improved glycaemic control and lower perioperative complications. ${ }^{[807]}$ Continuing at least part of the basal insulin is the reasonable, physiologic approach to controlling glucose levels before surgery in patients with diabetes. Basal bolus regimens are also associated with reduced postoperative complications and reduced inpatient costs per day. The dose of usual basal insulin can be reduced by $20-30 \%$ if patient reports nocturnal or fasting hypoglycaemic history. ${ }^{[817]}$ Long-acting insulins demonstrate lesser peaks and hence do not result in hypoglycaemia during fasting states. It is advised that long-acting insulins must be taken as close as possible to the usual time of injection, preoperatively. The intermediate-acting insulin neutral protamine Hagedorn (NPH) is usually given 2 times daily. Premixed insulins 


\section{Table 24: Preoperative assessments}

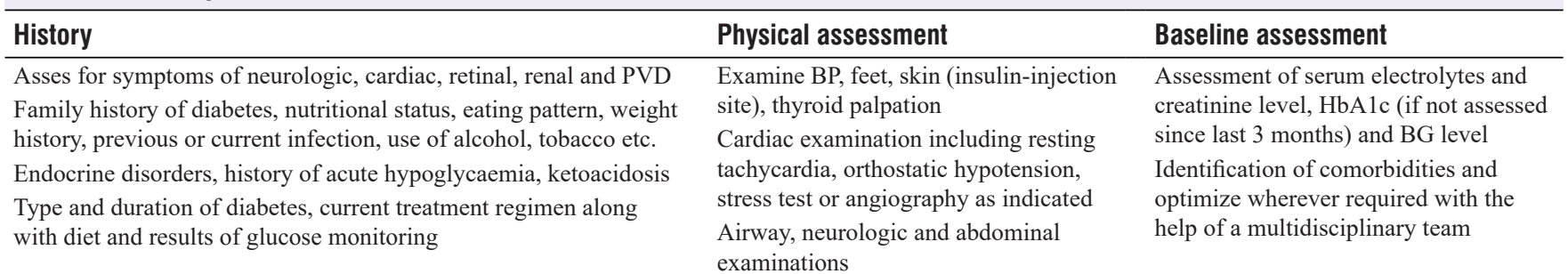

BP: Blood pressure, HbA1c: Glycosylated haemoglobin, PVD: Peripheral vascular disease, BG: Blood glucose

(combinations of basal and prandial insulin) are not prior to the surgery. ${ }^{[818]}$ Patients on insulin pumps subjected to longer surgical procedures should be shifted to the IV insulin infusion. Patient on basal-bolus insulin regimen should calculate the total daily insulin dose [Table 25]. ${ }^{[803]}$

\section{Intraoperative management}

Endocrine Society and Society for Ambulatory Anesthesia (SAMBA) recommend that intraoperative glucose levels be maintained less than $180 \mathrm{mg} / \mathrm{dL}$. Glucose levels should be monitored hourly intraoperatively and immediately after surgery. ${ }^{[812,819]}$ For patients with T2DM undergoing major or minor surgery IV infusion of insulin, glucose and potassium is recommended to maintain the glycaemic targets [Table 26]. ${ }^{[802]}$ To maintain the glucose targets intraoperatively, IV insulin infusion regimen-a protocol-driven algorithm is recommended. ${ }^{[806]}$

\section{Postoperative management}

Glucose control in noncritically-ill, non-ICU surgical patients is managed with subcutaneous insulin. During recover, the glucose levels must be monitored for at least every 2-h for all diabetic patients and for nondiabetics treated with insulin in the operating room. Correctional subcutaneous rapid-acting insulin doses are provided for BG greater than $180 \mathrm{mg} / \mathrm{dL}$ Patient should be transitioned to subcutaneous basal/bolus insulin regimen as soon as the patient is able to consume solid food. To prevent the insulin coverage gap while transitioning from IV infusion to subcutaneous, after the administration of first subcutaneous insulin dose there should be infusion overlap for at least 1-2-h. Patients previously on insulin regimen can continue their regular dose provided they are good with eating patterns. For patients not on insulin treatment previously, depending on patient's sensitivity to insulin calculate a subcutaneous regimen by totalling $0.2-0.5 \mathrm{U} / \mathrm{kg}$ of body weight. The total calculated daily insulin dose is to be divided as $50 \%$ basal component (long-acting insulin) $+50 \%$ prandial boluses (rapid-acting insulin) and split between breakfast, lunch and dinner. In patients treated with oral/non-insulin injectable initiate their regular home regimen provided they are eating regularly and are medically stable. Do not resume metformin for at least 2-3 days, especially in patients with

\begin{tabular}{|c|c|c|c|}
\hline$B G(m g / d L)$ & Usuala $^{\mathrm{a}}$ & Insulin-sensitive ${ }^{b}$ & Insulin-resistant ${ }^{c}$ \\
\hline$>141-180$ & 4 & 2 & 6 \\
\hline $181-220$ & 6 & 4 & 8 \\
\hline $221-260$ & 8 & 6 & 10 \\
\hline $261-300$ & 10 & 8 & 12 \\
\hline $301-350$ & 12 & 10 & 14 \\
\hline $351-400$ & 14 & 12 & 16 \\
\hline$>400$ & 16 & 14 & 18 \\
\hline
\end{tabular}

Numbers in each column represent the number of regular or rapid-acting insulin analogs per dose. Add the "supplemental" dose to the scheduled insulin dose. a ${ }^{\text {a }}$ iven before each meal and at bed-time for the patients able to take all or most of his meals, bStart regular insulin every $6 \mathrm{~h}$ or rapid acting insulin every $4-6 \mathrm{~h}$ for the patients who are elderly, not eating and with impaired renal function, 'In patients receiving more than $80 \mathrm{U} /$ day before admission and those who were receiving corticosteroids. BG: Blood glucose

\section{Table 26: Intravenous insulin infusion protocol}

Initiate insulin infusion by mixing $100 \mathrm{U}$ short-acting insulin +100 $\mathrm{mL}$ normal saline at the rate of $0.5-1 \mathrm{U} / \mathrm{h} \quad(0.5-1 \mathrm{~mL} / \mathrm{h})^{\mathrm{a}}$. Initiate separate infusion of $5 \%$ dextrose + water at the rate of 100-125 $\mathrm{mL} / \mathrm{h}$. Monitor BG every hour (every $2 \mathrm{~h}$ when stable) and according to the following algorithm adjust the insulin infusion rate

\begin{tabular}{|c|c|}
\hline $\begin{array}{l}\text { BG level } \\
\text { (mg/dL) }^{b}\end{array}$ & Action \\
\hline$<70$ & $\begin{array}{l}\text { Recheck BG after turning off infusion for } 30 \mathrm{~min} \text {. If } \\
\text { reading still shows }<70 \mathrm{mg} / \mathrm{dL} \text {, give } 10 \mathrm{~g} \text { glucose and } \\
\text { keep checking BG every } 30 \mathrm{~min} \text { until the level rises to } \\
100 \mathrm{mg} / \mathrm{dL} \text {, resume the infusion and reduce rate by } 1 \mathrm{U} / \mathrm{h}\end{array}$ \\
\hline $70-120$ & Reduce insulin infusion rate by $1 \mathrm{U} / \mathrm{h}$ \\
\hline $120-180$ & Continue the regular insulin infusion \\
\hline $181-250$ & Increase rate of insulin infusion by $2 \mathrm{U} / \mathrm{h}$ \\
\hline $251-300$ & Increase rate of insulin infusion by $3 \mathrm{U} / \mathrm{h}$ \\
\hline $301-350$ & Increase rate of insulin infusion by $4 \mathrm{U} / \mathrm{h}$ \\
\hline $351-400$ & Increase rate of insulin infusion by $5 \mathrm{U} / \mathrm{h}$ \\
\hline$>400$ & Increase rate of insulin infusion by $6 \mathrm{U} / \mathrm{h}$ \\
\hline
\end{tabular}

af hypoglycaemic condition persists rate of glucose infusion can also be increased, ${ }^{\mathrm{b}} 120-180 \mathrm{mg} / \mathrm{dL}$ is the target $\mathrm{BG}$ range. BG: Blood glucose

renal dysfunction, hepatic impairment or heart failure because of potential risk of metabolic acidosis..$^{[806,812]}$ 


\section{Type 2 Diabetes Mellitus and Pregnancy}

\section{ReCOMmEndations}

\section{Recommended Care}

- Preconception care should be introduced in the routine diabetes care and maternal complications should be addressed

- All diabetic women of child bearing potential should be educated about risks of unplanned pregnancy and its outcomes, use of contraceptives and family planning

- Need for strict glycaemic control, use of insulin, maintenance of safe levels of HbAlc, i. e. $6.5 \%-7.0 \%$ to minimize risk of neonatal complications must be explained

- Insulin is the first line therapy to treat hyperglycaemia in pregnant women with pre-existing diabetes. Replace all the other anti-glycaemic medication with insulin treatment

- If adequate blood glucose levels not achieved during pregnancy with multiple daily insulin infusions, insulin pump therapy for safe treatment should be advised

- Educate about teratogenic effects of ACEi, ARBs, atenolol and statins

- A dose of $400 \mu \mathrm{g} /$ day of folic acid should be recommended to avoid neural tube defects

- Antepartum care:

- During first 10 weeks of pregnancy, offer retinal and renal assessment if not assessed

- Offer ultrasound monitoring during week 16-32 to detect foetal growth and to detect structural abnormalities, if any

- Intrapartum care:

- Capillary blood glucose level should be within the optimum level of $70-110 \mathrm{mg} / \mathrm{dL}$ during labour

- Appropriate dose of regular insulin with dextrose infusion must be preferred to achieve target glycaemic levels during labour

- Postpartum care:

- Monitor blood glucose level, consider insulin dose reduction/stoppage to avoid hypoglycaemia. Reassessment of glycaemic status at 6 weeks postpartum with a 75 gm OGTT

- Reminder about importance of contraceptives in women with pre-existing diabetes and pre-conception care and planning for pregnancies in future

\section{Limited Care}

- Multi-disciplinary clinic constituting perinatologist, dietician, endocrinologist, obstetrician, diabetes counsellor should be developed for ideal management of diabetes

- Monthly assessments of HbA1c level should be carried out. SMBG level at fasting, pre and post-prandial intervals to attain optimum glycaemic control

\section{BACKGROUND}

Poorly controlled diabetes in women before conception can lead to severe birth defects in 5\%-10\% of pregnancies, and can lead to spontaneous abortion in $15 \%-20 \%$ of pregnancies. ${ }^{[820]}$ Despite the alarmingly high prevalence of diabetes in India, studies on the effects of T2DM in pregnancy and its outcomes are limited. The overall prevalence of pre-gestational diabetes has been recorded to be doubled from $1999-2005 .{ }^{[821]}$ Recent studies have revealed that the prevalence of diabetes in pregnant women with pre-existing diabetes as $3.4 \%-3.8 \%$, of which the majority were suffering from T2DM. ${ }^{[822,823]}$ Preexisting diabetes can have deleterious effects on pregnancy as it leads to complications for both mother and the foetus. These complications are associated with hazardous pregnancy outcomes including still-birth, spontaneous abortion, preeclampsia, perinatal mortality, low birth weight, respiratory distress, neonatal death, neonatal hypoglycaemia etc. ${ }^{[824-826]}$

Though there are several management protocols available for GDM, limited resources for the management of pregnancy in patients with pre-existing T2DM is available. Since it involves various stages of pregnancy, a multidisciplinary approach must be adopted.

\section{Preconception planning and care [Table 27]}

Before conception, a set of treatment regimens that aim at optimization of social, biomedical and psychological aspects in a woman with pre-gestational diabetes (T1DM and T2DM) is referred to as pre-conception management. ${ }^{[827]}$ In order to minimize the diabetic pregnancy complications and to control congenital malformation it is essential to introduce preconception care in primary care plan for women with child bearing potential. ${ }^{[828]}$

Along with early diagnosis and glycaemic controls, proper nutritional diet, and regular follow-up of metabolic stress and complications can help in a successful pregnancy in patients with diabetes. Introduction of multidisciplinary clinics in management of diabetic pregnancy can reduce the rate of perinatal mortality and can also improve neonatal care. ${ }^{[829]}$

\section{Counselling}

The pre-conception counselling process should be discrete, concise and considerate and must provide clear explanation about sensitivity to social and moral conventions. Women with pre-existing diabetes should be counselled about the need for contraception while on treatment with insulin. Educate diabetic women in their reproductive age about the 
Table 27: Elements of preconception plan

\begin{tabular}{|c|c|c|c|}
\hline Counselling & Assessment of medication & Glycemic control & Supportive investigation and management \\
\hline Need for contraception and effective & Potentially teratogenic drugs & Risk of hypoglycaemia & Optimum HbA1c level \\
\hline measures & Use of oral hypoglycaemic & Risk of maternal and & Urine albumin: Creatinine ratio \\
\hline Risk associated with unplanned pregnancy & agents & foetal complications due to & Test for HIV, HBV, HCV, VDRL \\
\hline Financial/family planning & Insulin therapy & hyperglycaemic condition & rubella, TSH and fundus \\
\hline $\begin{array}{l}\text { Need for strict glycaemic control and } \\
\text { insulin }\end{array}$ & Use of insulin analogs & $\begin{array}{l}\text { Educate on self-monitoring } \\
\text { of BG }\end{array}$ & \\
\hline
\end{tabular}

HbA1c: Glycosylated haemoglobin, BG: Blood glucose, HIV: Human immuno-deficiency virus, HBV: Hepatitis B virus, HCV: Hepatitis C virus,

TSH: Thyroid stimulating hormone

issues associated with unplanned pregnancy. Patients must be counselled and prescribed appropriate contraceptive measures that must be adapted until the metabolic parameters are appropriate to conceive. Since the primary goal for glycemic management in the preconception period and during the first trimester is to obtain the lowest $\mathrm{HbAlc}$ levels possible without hypoglycaemia, women should be made aware that they can have a planned conception only with HbAlc less than $6.5-7.0 \%$ to lower the risk of congenital anomalies. ${ }^{[827,829]}$ Crirtical complications with T2DM such as hypertension, intra uterine growth retardation and risk of obesity along with their preventions and management should be explained to the patients during pre-conception counselling. ${ }^{[827]}$ Muslim pregnant women with pre-existing T2DM must be advised to avoid fasting during the month of Ramadan. However, religious fasting are personal decisions, and a practical approach should be explained with emphasis on the risks to the mother and the foetus. ${ }^{[609]}$ Pre-conception counselling must aim at minimizing the risk of pregnancy complications in girls and women in their reproductive age with diabetes. Such counselling can improve the health of the mother and reduce cost burdens for the mother and the child. ${ }^{[830]}$

\section{Glycaemic target-preconception and antepartum [Tables 28 and 29]}

It is recommended that women with T2DM who are actively trying to become pregnant should be switched from oral or noninsulin injectable hypoglycemic agents to insulin prior to conception if possible during preconception and the first trimester of women with pre-gestational diabetes the primary goal is to maintain optimum glycaemic level. Effective measures must be taken to maintain the ideal glycaemic value while minimizing the risk of hypoglycaemia. The IDF and ADA recommend a pre-conception $\mathrm{HbA} 1 \mathrm{c}$ level of $<7 \%$, whereas as per the National Institute for Health and Clinical Excellence (NICE) recommendation, the $\mathrm{HbAlc}$ level can be lower i. $\mathrm{e}<6.5 \%$, provided it is safely achieved. ${ }^{[831]}$ In order to prevent chances of spontaneous abortions and major congenital malformations, target $\mathrm{HbA} 1 \mathrm{c}$ must be as close to normal as possible without significant hypoglycaemia. ${ }^{[832]} \mathrm{HbA} 1 \mathrm{c}$ should be assessed monthly due to the changing kinetics of RBCs and physiological alterations in glycemic aspects during preconception and in pregnant women with diabetes as it proves to be helpful in planning therapy. ${ }^{[827,830]}$ The ADA recommends $\mathrm{HbA} 1 \mathrm{c}$ testing to be done at the time of fasting, pre-parandial and post-parandially in pregnant women with diabete ${ }^{[830]}$ In women with pre-existing diabetes, provision of basal and prandial insulin needs with intensified insulin regimens (multiple dose regimens of subcutaneous long-and short-acting insulins) are known to give best results. Rapid acting insulin dosage (pre meal) can be adjusted using insulin pumps or basal-bolus therapy for which preprandial glucose monitoring is essential. Monitoring of postprandial blood glucose aids in lowing the risk of preeclampsia.

\section{Optimization of antidiabetic regimes}

No oral OADs are approved for pre-existing diabetes in pregnancy although glyburide and metformin have been used in multiple RCTs for GDM. Minimal data on thiazolidinediones or metiglinides and no data on incretin-based DPP-4 inhibitors and GLP-1 analogues are available. ${ }^{[833]}$ Metformin and glyburide may be used during pregnancy but these drugs cross the placental barrier and hence should be replaced with insulin therapy at the earliest. ${ }^{[827,831,833]}$ Potential concerns for SGLT2 inhibitors in pregnancy due to profound polyuria in a pregnant patient with familial renal glycosuria have been reported and since pregnancy causes polyuria and glycosuria normally due to increased glomerular filtration rate so SGLT2inhibitors are not be expected to be beneficial. ${ }^{[833]}$ Insulin, does not cross the placenta, and hence is the first choice to attain targeted glycaemic goal in pregnant women with pre-existing diabetes. ${ }^{[827,830]}$ Insulin requirements may increase as the pregnancy progresses, and the requirement peaks between 28 weeks and 32 weeks of gestation. ${ }^{[834]}$ Considering alteration in physiology of pregnant women, daily SMBG is required more frequently and insulin dose must be optimized at different stages of pregnancy as per requirement. ${ }^{[830]}$ Compared to T1DM, management of glucose level is easier in T2DM, however there might be requirement of higher dose of insulin as $>150$ units/ day i. e. approximately $50 \%$ elevation. ${ }^{[801]}$ Insulin pump therapy is also considered to be beneficial in maintaining $\mathrm{HbAlc}$ level in pregnant women with pre-gestational diabetes, without any increase in risk of hypoglycaemia. However, cost and the risk for marked hyperglycaemia or DKA as a consequence of insulin delivery failure from could be an issue. ${ }^{[833]}$

Pre-gestational diabetics are at a high risk of preeclampsia, hence the American College of Obstetricians and Gynecologists recommends use of low-dose aspirin $(81 \mathrm{mg} /$ day) prophylaxis to be initiated between 12 weeks and 28 weeks of gestation (ideally before 16 weeks of gestation) and continued until delivery to 
prevent preeclampsia. ${ }^{[834]}$ Such preventive action can lower the rates of morbidity and health care costs of the neonate. ${ }^{[830,831]}$

\section{Screening and management for diabetes complications [Table 30]}

Early screening of diabetes complications like retinopathy, neuropathy, heart failure, chronic kidney disease in pre-conception period is essential as they can be life-threatening and associated with lower quality of life for both the mother and the foetus if not diagnosed or treated at an early stage. Poorly controlled pre-gestational diabetes may lead to serious end-organ damage that may result into life threatening conditions. These complications can be controlled or prevented with appropriate diabetes management. ${ }^{[831]}$ Diabetic retinopathy is the leading cause of blindness, and women with diabetes who become pregnant should have a comprehensive eye examination in the first trimester and should be monitored closely throughout pregnancy. ${ }^{[835]}$ Diabetic nephropathy is estimated to be present in 5-10\% of diabetic pregnancies ad progression to end stage renal disease have been reported in several women. Also, women with pre-existing diabetic nephropathy are at significantly higher risk for obstetric complications, such as hypertension, uteroplacental insufficiency, and iatrogenic preterm birth because of worsening renal function. ${ }^{\left[{ }^{836]}\right.}$ Hypertension, especially in the presence of nephropathy, increases the risk of preeclampsia, uteroplacental insufficiency, and stillbirth. ${ }^{[837]}$

Table 28: Glycaemic target in women with preexisting type 2 diabetes mellitus before and during pregnancy

\begin{tabular}{lc}
\hline Condition & Glycaemic target \\
\hline Fasting & $95 \mathrm{mg} / \mathrm{dL}(5.3 \mathrm{mmol} / \mathrm{L})$ \\
1-h postprandial & $140 \mathrm{mg} / \mathrm{dL}(7.8 \mathrm{mmol} / \mathrm{L})$ \\
2-h postprandial & $120 \mathrm{mg} / \mathrm{dL}(6.7 \mathrm{mmol} / \mathrm{L})$ \\
\hline
\end{tabular}

Pre-gestational diabetes is a risk factor for acute myocardial infarction during pregnancy and hence pregnancy may be contraindicated in patients with pre-existing coronary artery disease due the hemodynamic changes that may occur during pregnancy and may cause myocardial infarction and death. ${ }^{[832]}$ Recalcitrant nausea and vomiting due to gastroparesis are result of diabetic neuropathy in pregnant women. Gastroparesis impacts interaction between diet and diabetes regimens, and complicates glycaemic control thereby increasing the risk of hypoglycaemic episodes. ${ }^{\left[{ }^{[34]}\right.}$ Diabetic ketoacidosis is a lifethreatening emergency observed in $5-10 \%$ of all pregnancies complicated by pre-gestational diabetes. Abdominal pain, nausea and vomiting, and altered sensorium are the common clinical presentations. Hypoglycemia and hypokalemia are frequent complications of diabetic ketoacidosis therapy, hence, glucose and potassium concentrations should be monitored closely. ${ }^{[220,838]}$

Optimization of anti-hypertensive medications [Table 31] Anti-hypertensive medication therapy with ACE inhibitors and angiotensin receptor blockers (ARBs) are contraindicated in women with pre-existing diabetes and planning for pregnancy as these medications are teratogenic and can cause intra-uterine growth retardation, foetal renal dysplasia and oligohydramnios. ${ }^{[803,827,830,831]}$ A large randomized controlled trial in pregnant women with pre-existing or gestational hypertension showed that targeting a diastolic blood pressure (BP) of $85 \mathrm{mmHg}$ vs. $100 \mathrm{mmHg}$ reduced neonatal respiratory complications and rates of severe maternal hypertension (i. e. $>160 / 110 \mathrm{mmHg}$ ) ${ }^{[839]}$ Labetalol, methyldopa, diltiazem, nifedipine, clonidine and prazosin are considered to be safe anti-hypertensives during pregnancy. Use of atenolol is not recommended in pregnancy. Use of chronic diuretics as antihypertensives are also not recommended as they are associated with restriction of maternal plasma volume that leads to

Table 29: Safety of medicines for diabetes before and during pregnancy

Noninsulin glucose-lowering agents

\begin{tabular}{|c|c|c|}
\hline & Compound & Effects on pregnancy \\
\hline \multicolumn{3}{|l|}{ Class } \\
\hline \multirow[t]{3}{*}{ SU } & Glimepiride & Intrauterine death, skeletal deformities and foetal growth retardation \\
\hline & Glipizide & Crosses placental barrier \\
\hline & Glibenclamide & Small amount may cross placental barrier, may reduce rate of morbidity and mortality in foetus and infant \\
\hline Biguanide & Metformin & $\begin{array}{l}\text { Crosses placental barrier and shows congenital malformation however lower in rate than those not on } \\
\text { metformin medication }\end{array}$ \\
\hline$\alpha$-glucosidase inhibitors & Acarbose & Study depicted few babies with congenital malformation \\
\hline Meglitinides & $\begin{array}{l}\text { Nateglinide } \\
\text { repaglinide }\end{array}$ & $\begin{array}{l}\text { Transfusion through placental barrier is unknown yet suggested that may produce risk of developmental } \\
\text { toxicity in foetus at a lower risk }\end{array}$ \\
\hline \multirow[t]{2}{*}{ TZDs } & Pioglitazone & Crosses placenta, delayed foetal development, reduced foetal weight and postimplantation losses \\
\hline & Rosiglitazone & Crosses placenta, causes foetal growth retardation, foetal death and also placental toxicity \\
\hline \multicolumn{3}{|l|}{ Insulins } \\
\hline \multirow[t]{3}{*}{ Rapid acting analog } & \multirow{3}{*}{$\begin{array}{l}\text { Aspart, lispro and } \\
\text { gluisine, glargine } \\
\text { or detemir }\end{array}$} & $\begin{array}{l}\text { Insulin aspart known be most effective in management of glycemic control without causing the risk of } \\
\text { hypoglycaemia during preconception and throughout pregnancy }\end{array}$ \\
\hline & & Lispro was found to be safe and effective in maintaining BG level \\
\hline & & No safety and efficacy data available on use of detemir or glargine and glusine in pregnancy \\
\hline
\end{tabular}




\section{Table 30: Screenings and management for diabetes complication}
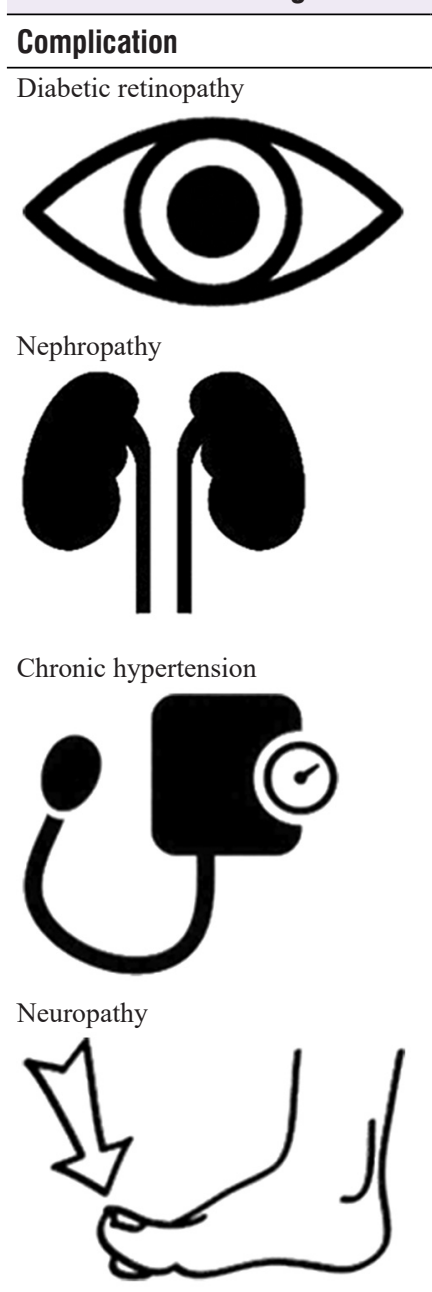

Diabetic ketoacidosis

\section{Screening and management}

Screening of retina during preconception and more frequently during pregnancy and up to 1 year postpartum is essential for early detection and implementation of preventive measure

In patients with preexisting retinopathy ensure appropriate treatment to avoid disease progression

Tight glycemic control is the primary measure to prevent retinopathy other measures to treat retinopathy are laser coagulation treatment, vitrectomy, VEGF drugs and intravitreal steroid injections

Counsel women who are planning pregnancy about the risk of diabetic retinopathy and if existing then prevention of its progression

Diagnosis of BP at every visit should be monitored as perinatal complications like preeclampsia, intra-uterine growth retardation and preterm birth in diabetic nephropathy are very common

Patient with preexisting nephropathy should be monitored closely to maintain the albuminuria and medication started to decrease the renin-angiotensin-aldosterone-system activity as varying renal function deterioration is expected during pregnancy

Referral to nephrologist should be considered if there is abnormal serum creatinine level i.e., $\geq 120 \mu \mathrm{mol} / \mathrm{L}$, albumin: Creatinine ratio being $>30 \mathrm{mg} / \mathrm{mmol}$ of or eGFR $<45 \mathrm{~mL} / \mathrm{min} / 1.73 \mathrm{~m}^{2}$

As complication risks are comparatively high in diabetic patients who undergo kidney transplantation, patient counselling and clinical decision making of conception are to be considered

Screening of BP at every visit is essential to avoid any maternal or foetal complications. Guidance on self-monitoring of BP should be provided

During preconception counselling, patient with preexisting diabetes and hypertension should be made aware of the teratogenic anti-hypertensive medications to avoid during pregnancy

ACEIs, ARBs and spironolactone are contraindicated and hence should be discontinued before pregnancy as these anti-hypertensive drugs are teratogenic

In patients with preexisting hypertension and on anti-hypertensive medication, in order to optimize long-term health of the mother and reduce risk of foetal complications ADA suggests the target BP to be 120-160 (systolic) and $80-105 \mathrm{mmHg}$ (diastolic)

Educate patient with preexisting diabetes and planning for pregnancy, about the diabetic foot hygiene, nail cutting, appropriate footwear to prevent the risk of diabetic foot

Feet inspection in every month is recommended for patients with preexisting diabetes but at every visit for patients at high ulceration risk

Maintaining optimum $\mathrm{HbAlc}$ level $<7 \%$ can lead to reduction in risk of neuropathy by $35 \%$

Arterial blood gases to ascertain degree of acidosis must be conducted. Glucose must be continuously monitored along with capillary BG ketones, and serum ketones

Aggressive hydration and IV insulin and the most important treatment regimes

IV fluids: Isotonic sodium chloride may be used, with total replacement of 4-6 L

Bicarbonates may be used based on the $\mathrm{pH}$ levels

BP: Blood pressure, VEGF: Vascular endothelial growth factor, EGFR: Estimated glomerular filteration rate, ACEIs: Angiotensin converting enzymes, ARBs: Angiotensin receptor blockers, ADA: American Diabetes Association, HbA1c: Glycosylated haemoglobin, IV: Intravenous

reduction in utero-placental perfusion. ${ }^{[827]}$ Severe preeclampsia and acute-hypertension management may be treated with vasodilator like hydralzine during pregnancy.

\section{Management of dyslipidaemia}

Dyslipidaemia identified during pregnancy should be treated with diet and exercise intervention, as well as glycaemic control if indicated. A lipid profile at preconception in women with familial hypercholesterolemia (FH) must be conducted and a target level of LDL cholesterol, HDL cholesterol and triglycerides as $<100 \mathrm{mg} / \mathrm{dL}(2.6 \mathrm{mmol} / \mathrm{L}),>35 \mathrm{mg} / \mathrm{dL}$ $(0.905 \mathrm{mmol} / \mathrm{L})$ and $<105 \mathrm{mg} / \mathrm{dL}(1.7 \mathrm{mmol} / \mathrm{L})$, respectively must be established for each patients. ${ }^{[840]}$ The use of statins is contraindicated during pregnancy due to teratogenicity. There is limited data on the teratogenicity of lipid-lowering agents, ezetimibe, nicotinic acid, and fibrates in humans. ${ }^{[841]}$ Bile acid binding resins, cholestyramine and colsevelam, are the only medications currently acceptable to use during pregnancy as they do not pass into the systemic circulation ${ }^{[842]}$ Fenofibrate and gemfibrozil are pregnancy category $\mathrm{C}$ drugs and should only be used if the potential benefit justifies the potential risk to the fetus. Initiate treatment with fibrate if the triglyceride level is more than the optimum level of $>400 \mathrm{mg} / \mathrm{ml}$ (fasting condition) so as to minimize the occurrence of pancreatitis. ${ }^{[822]}$

\section{Antepartum care}

\section{Folic acid supplementation}

Periconceptional folic acid supplementation decreases the occurrence and recurrence of neural tube defects (NTDs). Hence, in the preconception counselling, patients should 


\begin{tabular}{lll}
\hline \multicolumn{2}{l}{ Table $31:$ Safety of medicines for complications of diabetes before and during pregnancy } \\
\hline Class & Compound & Effects on pregnancy \\
\hline ACEIs & $\begin{array}{l}\text { Lisinopril, perindropril, enalapril, } \\
\text { moexipril, trandolapril and quinapril }\end{array}$ & $\begin{array}{l}\text { Usage of ACEI and ARBs to treat hypertension should be avoided during } \\
\text { pregnancy as they severely affects the control over renal function and also } \\
\text { foetal and neonatal BP. They may also cause oligohydramnios and skull defects }\end{array}$ \\
ARBs & Losartan, telmisartan & $\begin{array}{l}\text { Usage of statins in reduction of elevated levels of cholesterol should be avoided } \\
\text { in pregnancy as well as in lactation as they may cause congenital malformation }\end{array}$ \\
Statins & Atorvastatin, rosuvastatin, fluvastatin & $\begin{array}{l}\text { Obesity treatment in pregnancy with orlistat shows low risk to foetus and hence } \\
\text { should be used with caution during pregnancy }\end{array}$
\end{tabular}

be educated on folic acid requirement. ${ }^{[843]}$ Women with preexisting diabetes who are planning to become pregnant must be advised to take folic acid ( $5 \mathrm{mg}$ /day) until 12 weeks of gestation to reduce the risk of having a baby with a neural tube defect. ${ }^{[831]}$

\section{Nutrition therapy and weight gain targets}

The primary aim of nutritional therapy in pregnancy is to provide calories to maintain optimum glycaemic control for normal growth and development of the foetus with normalizing dyslipidaemia. As a consequence of physiologic changes that follow pregnancy, caloric requirements are increased during the second and third trimesters. ${ }^{[18]}$ Wholesome food choices with $40-50 \%$ calories from complex, high-fibre carbohydrates, $15-30 \%$ calories from protein, and 20-35\% calories from primarily unsaturated fats) are commonly advised. ${ }^{[834]}$ To fulfil the addiotnal dietary needs, diets often altered or modified for the amount and type of carbohydrates to be consumed during pregnancy. It is advisable to include diet rich in omega- 3 fatty acids and non-starch polysaccharides with low glycemic index, and avoid excess intake of saturated fats and TFAs that can lead to increased risk of complications. High-carbohydrate lowfat diet such as legumes, unprocessed fruits and vegetables should be included in the diet. Vitamin D supplementation $(10 \mu \mathrm{g} /$ day $)$ are prescribed in women with risk of vitamin $\mathrm{D}$ deficiency during pregnancy. Folic acid supplementation with a recommended dose of $400 \mu \mathrm{g} /$ day is prescribed until 12 weeks of pregnancy to prevent risk of neural tube defects. Vitamin A supplementation, liver and liver products rich in vitamin A should be avoided as they may be teratogenic. Iron supplements are not often prescribed in pregnancy as they might be associated with unpleasant maternal side-effects. ADA suggests the Dietary Reference Intakes (DRI) to be $>175 \mathrm{~g}$ of carbohydrate, $>71 \mathrm{~g}$ of protein and about $28 \mathrm{~g}$ fiber in all pregnant women. ${ }^{[801,831,830,832]}$

\section{Weight management}

Obesity is the major complication in women with pre-existing T2DM and hence, monitoring and weight management is important to avoid CV risk in pregnancy. ADA recommends the weight gain of overweight women during pregnancy should be 15-25 $\mathrm{lb}$ whereas for obese women it should be 10-20 lb. ${ }^{[830]}$ Maintenance of weight gain targets during pregnancy can be easily done with the help of appropriate dietary plan along with life-style interventions. Yoga either individually or combined physical activity has been remarkably helpful in weight management. Orlistat a lipase inhibitor shows low risk to foetal development and hence obesity/overweight in pregnancy can be treated with orlistat with caution and close monitoring. Orlistat is also known to improve ovulation as it acts indirectly by weight reduction and improves ovulation in overweight and obese subfertile women as compared to lifestyle modifications. ${ }^{[844]}$ Orlistat is the only approved and available drug in India for modest weight loss in combination with lifestyle interventions. ${ }^{[845]}$ (Refer section 'Obesity and Type 2 Diabetes Mellitus' for more information).

\section{Intrapartum care \\ Glycaemic targets during labour and delivery}

The timing and mode of birth must be discussed during antenatal appointments, especially during the third trimester. If there are metabolic or any other maternal or foetal complications, elective birth before 37 weeks for women with type 1 or type 2 diabetes must be considered. ${ }^{[831]}$ Studies have suggested that the blood glucose target should be maintained $100-126 \mathrm{mg} / \mathrm{dL}$ to prevent hypoglycaemia in neonates. It was found that neonatal hyperglycema is at higher risk when the maternal blood glucose level reaches to $>180 \mathrm{mg} / \mathrm{dL} \cdot{ }^{\left[{ }^{846]}\right.}$ In a retrospective analysis including 137 singleton cases, mothers with blood glucose level of about $72-144 \mathrm{mg} / \mathrm{dL}(4-8 \mathrm{mmol} / \mathrm{L})$ resulted in $87 \%$ $(n=26)$ neonatal hypoglycaemia, of which 13 neonates were admitted to ICU. These 13 neonates were born with maternal blood glucose level $>144 \mathrm{mg} / \mathrm{dL}(8 \mathrm{mmol} / \mathrm{L})$. Thus, blood glucose must be monitored closely and controlled within the targets. ${ }^{[847]}$ The capillary plasma glucose must be monitored every hour during labour and birth in women with diabetes, and ensured that it is maintained between $70-110 \mathrm{mg} / \mathrm{dL}$ (3.9-6.1 $\mathrm{mmol} / \mathrm{L}$ ) in women with pre-existing T2DM. ${ }^{[831]}$ Monitoring should be carried out 2-h to 4-h during the latent stage, active stage requires monitoring every $1-2 \mathrm{~h}$ and every hour in patient on glucose infusion. During labor, women with pre-gestational diabetes generally should undergo continuous intrapartum electronic foetal monitoring. ${ }^{[834]}$ To achieve target glycaemic levels, IV dextrose and insulin infusion during labour and birth for women with diabetes whose capillary plasma glucose is not 
maintained between 70-110 mg/dL may be considered. Rapid acting insulin analog like aspart or lispro are the preferred choice in achieving target glycemic value as they minimize the risk of hypoglycaemia. Use of oral anti-diabetics during intrapartum is not permitted as patients are on insulin or glucose infusion during that phase. Also FDA does not approve use of oral anti-diabetics during pregnancy ${ }^{[830]}$

\section{Postpartum management Care of newborn}

Neonates born to women with pre-existing T2DM are at a higher risk of morbidities like macrosomia, hypoglycaemia, respiratory distress, cardiomyopathy, hematologic disorders and hypocalcaemia. ${ }^{[848]}$ It is recommended to admit babies showing signs of above morbidities to the NCU immediately postpartum for proper care and management. ${ }^{[834]}$ To minimize neonatal complication, proper diabetic management in the antenatal period is required and delivery should be accompanied by experienced paediatricians. ${ }^{[827]}$

\section{Glycaemic control}

Insulin requirement falls in the postpartum period by $34 \%$ than that required in preconception period. Over the next 1-2 weeks of postpartum the insulin requirement returns back to that required during pre-conception period. Women on insulin should be closely monitored to avoid the risk of hypoglycaemia. Monitor maintenance of pre-feed capillary plasma glucose level of the neonate and assure it to be minimum $2 \mathrm{mmol} / \mathrm{L}$.

\section{Lactation}

Breastfeeding should be encouraged in women with pregestational diabetes. There is a sharp decline in the insulin requirement after delivery and hence the dose of insulin needs to be adjusted accordingly. ${ }^{[827]}$ Dietary care to prevent risk of obesity and neonatal care is prime concern during lactation. Strict control over blood glucose level for women with pre-existing diabetes who underwent caesarean section is important to avoid infection injuries. During lactation, women with pre-existing diabetes can resume or continue to take metformin and glibenclamide immediately after birth, however should avoid use of medicines that were contraindicated during pregnancy, for treatment of diabetes and its complication, due to safety considerations. ACE inhibitors, ARBs, oral hypoglycemic agents, obesity medicines and statins should be avoided during breastfeeding. ${ }^{[831]}$

\section{Postpartum contraception}

One of the major barriers to effective preconception care is unplanned pregnancy. To minimize the risk of congenital malformation due pre-existing diabetes and its complications it is important to remind women in postpartum period about use of effective contraception and family planning. For women who do not choose permanent contraception with tubal ligation, long-acting reversible contraception with an intrauterine device or implantable progestin are the most effective forms of contraception and should be recommended..$^{[849]}$

\section{Type 2 Diabetes Mellitus and Critical llenesss}

\section{ReCOMMENDATIONS}

\section{Recommended Care}

- Maintain glycaemic target of 140-180 mg/dL in medically morbid patients and a target of 110-140 mg/dL in surgically morbid patients

- Intensive insulin therapy should be initiated when the blood glucose level is more than $180 \mathrm{mg} / \mathrm{dL}$

- Patients on continuous IV insulin infusion should be monitored frequently to avoid the risk of hypoglycaemia

- Monitor blood glucose on hourly basis and then every $2 \mathrm{~h}$ once blood glucose level is stable or 3 consecutive readings fall near the target glycaemic range

- Insulin in the form of continuous IV infusion is the only recommended route of administration in the critical care setting.

- Use of SSI, long or intermediate acting insulin regimens and premixed insulin, subcutaneously are not recommended

- On admission in the hospital, there should be mandatory testing of blood glucose, in $24 \mathrm{~h}$ at least two readings should be obtained

- CGMS should be preferred for glucose monitoring in critically ill patients

\section{Limited Care}

- Use validated written or computerized protocols for predefined insulin dosage based on glycemic fluctuations

- Hospitals should have a record of hypoglycaemic episodes and should be evaluated for root cause 


\section{BACKGROUND}

Risk of longer hospital stay, morbidity and mortality increases with the risk of hyperglycaemia in the ICU. There have been several discussions on the ideal glycaemic control in surgical, medical or cardiac ICUs but the results are controversial and the final outcome has not been attained.${ }^{[850]}$ Hospitalization of the diabetic patient interrupts the outpatient balance of medications, diet, and exercise, and may lead to hyperglycaemia or hypoglycaemia. ${ }^{[851]}$ Conversely, hyperglycaemia as a co-morbid condition increases the level of severity of primary illness and complicates the patient management. ${ }^{[852]}$ There have been many published guidelines on management of hyperglycaemia in hospitalized patients but with unclear biases involving nutritional, sociobehavioral, economic and cultural factors. Further, differences in racial, genetic and ethnic conditions impact insulin resistance, glucose and lipid metabolism which makes it difficult to apply western guidelines to Indian population. Hence in India there is an acute need to formulate relevant guidelines, friendly to both patient and physician to control hyperglycaemia in hospitalized diabetic patients. ${ }^{[853]}$

\section{Glycaemic targets in critically ill patients}

For the majority of critically ill patients with medical morbidity, ADA and AACE recommends the premeal glycaemic target to be between $110-130 \mathrm{mg} / \mathrm{dL}$ and the post meal $140-180 \mathrm{mg} / \mathrm{dL}(7.8-10.0 \mathrm{mmol} / \mathrm{L}) .{ }^{[854]}$ For patients with surgical morbidity, a goal of 110-40 mg/dL (6.1-7.8 $\mathrm{mmol} / \mathrm{L}$ ) is recommended if achievable without causing hypoglycaemia. ${ }^{[853]}$ The American College of Physicians recommends avoiding blood sugar levels below $140 \mathrm{mg} / \mathrm{dL}$, due to the associated risks of hypoglycaemia and glycaemic variability, in ICU patients and glycaemic target of $>180$ $\mathrm{mg} / \mathrm{dL}$ and less than $110 \mathrm{mg} / \mathrm{dL}$ are not recommended. ${ }^{[855]}$

\section{Moderate versus tight glycemic control in intensive care unit}

In critically ill patients, controlling blood glucose to the normal range as of healthy adult i. e $4.4-6.1 \mathrm{mmol} / \mathrm{L}$ with an aim to avoid hypoglycaemia is called tight glycaemic control (TGC). The Leuven trials and the normoglycaemia in intensive care evaluation-survival using glucose algorithm regulation (NICE-SUGAR) trial were conducted based on the TGC. Intensive insulin therapy to maintain blood glucose at or below $110 \mathrm{mg} / \mathrm{dL}$ is known to reduce morbidity and mortality among critically ill patients in the surgical intensive care unit. ${ }^{[856]}$ To maintain the patient in normoglycaemic range, the intensive insulin therapy was initiated when the blood glucose level reached renal threshold value usually $\geq 12 \mathrm{mmol} / \mathrm{L}$ in Leuven trial. Conversely in the NICE-SUGAR trial, intensive glucose control increased mortality among adults in the ICU: a blood glucose target of $<180 \mathrm{mg} / \mathrm{dL}$ resulted in lower mortality than a target of 81 to $108 \mathrm{mg} / \mathrm{dL}$ [Table 32]. ${ }^{[857]}$ Although the Leuven trial was associated with lower mortality rates, overall the TGC does not show any additional benefit.

\section{Glycaemic variability in critically unwell}

Glycaemic variability $(\mathrm{GV})$ in critically ill patients have a negative impact and increased rate of mortality along with sever hyperglycaemia. Risk of hypoglycaemia increases with the GV. Hence, clear understanding of GV is required to maintain glycaemic targets in the critically ill patients. It has been noted that there is an increase in insulin sensitivity over the duration of stay in ICU. During the first $24 \mathrm{~h}$ to $48 \mathrm{~h}$ the insulin requirement rapidly increases. At the onset of critical illness, patient being initially non-responsive to low intrinsic insulin sensitivity can lead to over administration of insulin. Thus, change in hormonal regulation increases risk of hypoglycaemia if TGC protocols are not taken into consideration. ${ }^{[856]}$

During the length of stay in ICU, patient's clinical assessment that includes fluctuation in glucose level, illness severity, concomitant medication that brings about GV (e. g glucocorticoids) and nutritional status should be carried out on daily basis to calculate insulin dosing [ADA 2019].

Insulin therapy in critically ill and route of administration OADs should be discontinued and their use avoided in the critical care setting. A continuous insulin infusion in the critically ill patient with T2DM may provide optimal glycaemic management. ${ }^{[851]}$ Insulin regimens should provide basal insulin and short-acting insulin to cover meal-related glucose excursions and to improve glycaemic control acutely. Unless the hyperglycaemia is mild and expected to be transient, regular insulin sliding scales should not be used alone in hospitalized patients. The IV infusion of insulin is initiated when the blood glucose level is $>180 \mathrm{mg} / \mathrm{dL}$. Insulin analogs like insulin aspart and insulin lispro as IV have less variability in pharmacokinetic properties and it is also easier to predict their absorption and duration of action, hence are more preferable than normal neutral protamine Hagedorn (NPH). Regular preprandial and postprandial insulins when replaced with theses rapid-acting insulin analogs can reduce the risk of hypoglycaemia. ${ }^{[856]}$

\section{Glucose monitoring in critically ill [Figure 17]}

Glucose measurement should be performed every 2 to $4 \mathrm{~h}$. If the serum glucose concentration are constantly fluctuating, it may be necessary to measure glucose every 30 or $60 \mathrm{~min}$. Approaches like venous, or whole blood, capillary blood and/ or plasma have been practiced for the accurate blood glucose monitoring. In India, capillary blood glucose assessment is the only approach followed till date. ${ }^{[858]}$ For patients who 
Table 32: Comparison between leuven and normoglycaemia in intensive care evaluation-survival using glucose algorithm regulation protocols, using Van den Berghe et al. (2009)

\begin{tabular}{lll}
\hline & Leuven & NICE-SUGAR \\
\hline Number of patients & 2748 & 6100 \\
Setting and sample (center) & $3 \times 1$ & 41 \\
Sample of ICU admissions (\%) & $68-95$ & 15 \\
Control comparison group (mmol/L) & $10-12$ & $7.8-10$ \\
Intervention target ${ }^{\dagger}(\mathrm{mmol} / \mathrm{L})$ & $<6.1$ & $<6.0$ \\
Insulin infusion & Continuous (central line) & Continuous and bolus: All routes \\
Feeding route for week 1 & Parenteral and enteral (fed) & Enteral only (hypocaloric) \\
BG target reached (\%) & 70 & $<50$ \\
Intergroup overlap in the Standard-deviation of BG measurements (\%) & $<10$ & $>50$ \\
Hypoglycaemic events & X6 & X13 \\
Morbidity & Less organ failure/infections & Negative \\
Mortality (\%) & Lowered by absolute 3 & Increased by absolute 3 \\
Therapy withdrawal & Late & Early \\
\hline
\end{tabular}

NICE-SUGAR: Normoglycaemia in intensive care evaluation-survival using glucose algorithm regulation, ICU: Intensive care unit, BG: Blood glucose

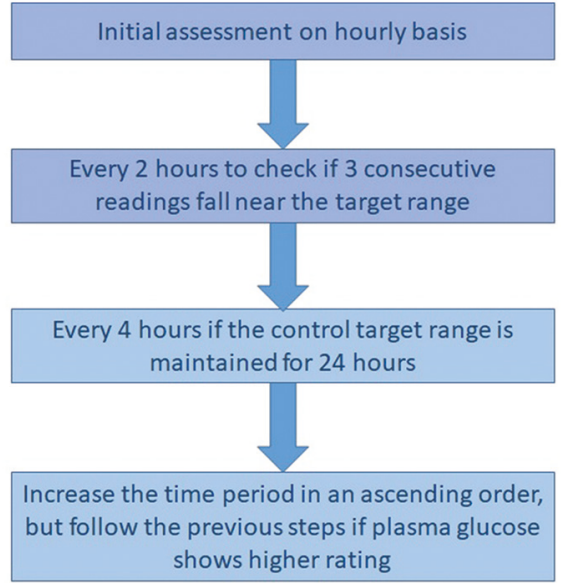

Figure 17: Point-of-care meters based monitoring of blood glucose in critically ill patients

are not on meals, the glucose monitoring is performed every 4-6 $\mathrm{h}$ whereas for those who take meals, preprandial glucose monitoring is preferred. Patients receiving intravenous insulin infusions require monitoring every 30 minutes to every 2-h. Point-of-care meters (POC) should be checked for its accuracy and approval status before use, to measure blood glucose in hospital settings.

Continuous Glucose Monitoring (CGM) helps to maintain the glycaemic target goals as they provide measurement of intermediate glucose levels frequently. CGM is advantageous over POC testing as they measure both magnitude as well as the direction of glucose trends which helps in minimizing the risk of hypoglycaemia. Compared to intermittent monitoring systems, CGM can offer benefit in the prevention of severe hyperglycemia and hypoglycemia by enabling insulin infusions to be adjusted more rapidly and potentially more accurately.

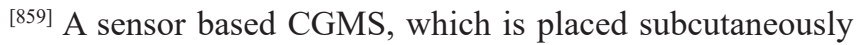
is a preferred method to monitor blood glucose in critically ill patient. ${ }^{[853]}$

\section{Hypoglycaemia in critical care}

Validated protocols to prevent and treat hypoglycaemia for inpatients should be adopted by each hospital. Blood glucose levels $<70 \mathrm{mg} / \mathrm{dL}(3.9 \mathrm{mmol} / \mathrm{L})$ should immediately be addressed in the nurse-initiated, standardised-hospitalwise protocols and also patient individual plans to prevent and treat hypoglycaemia. ${ }^{[854,860]}$ Information from patients with hypoglycaemic episodes should be recorded in the hospital records and tracked. Patient undergoing sudden dose reduction of corticosteroids, emesis, decreased oral intake, short/rapid-acting insulin showing inappropriate timings with regard to meals, interruption in oral, enteral or parenteral feedings and decreased rate of IV dextrose infusion may trigger iatrogenic hypoglycaemia. Prescribing appropriate medication for hypoglycaemia, proper treatment of first hypoglycaemic episode, suitable nutritional management and maintaining nutrition-insulin match throughout the hospital stay can help preventing iatrogenic hypoglycaemia. ${ }^{[860]}$ Proactive monitoring of glycaemic levels and data-driven glycaemic management approaches can serve as preventive measures for hypoglycaemia in the hospital. ${ }^{[807]}$ 


\section{Type 2 Diabetes Mellitus in Young and Adolescents}

\section{RECOMMENDATIONS}

\section{Recommended Care}

- Risk-based screening for prediabetes and/or T2DM should be considered in asymptomatic children and adolescents, performed after the onset of puberty or after 10 years of age, whichever occurs earlier.

- If tests are normal, repeat testing at a minimum of 3-year intervals, or more frequently if BMI is increasing.

- Fasting plasma glucose, 2-h plasma glucose during a 75-g oral glucose tolerance test, and HbAlc can be used to test for prediabetes or diabetes in children and adolescents.

- Panel of pancreatic autoantibodies tested to exclude the possibility of autoimmune T1DM.

- Treatment of youth-onset T2DM should include lifestyle management (long-term weight management, vigorous physical activity, healthy eating patterns), diabetes self-management education, self-monitoring of blood glucose, and pharmacologic treatment.

- A family-centred approach to nutrition and lifestyle modification is essential and nutrition recommendations should be culturally appropriate and sensitive to family resources.

- Bariatric surgery may be considered in adolescents with marked obesity (BMI: $>35 \mathrm{~kg} / \mathrm{m}^{2}$ ) and uncontrolled glycaemia and/or serious comorbidities despite lifestyle and pharmacologic intervention.

- Blood pressure should be measured and optimized to reduce risk and/or slow the progression of diabetic kidney disease.

- Youth with T2DM should be screened for the symptoms of other comorbidities including laboratory studies when indicated for neuropathy, retinopathy, non-alcoholic fatty liver disease, obstructive sleep apnoea, polycystic ovary syndrome (in female adolescents), cardiovascular disease, and dyslipidaemia.

- Starting at puberty, preconception counselling should be incorporated into routine diabetes clinic visits for all females of childbearing potential.

- Patients should be screened for smoking and alcohol use at diagnosis and regularly thereafter.

\section{BACKGROUND}

Until recently most children with diabetes had type 1 disease, however, prevalence of T2DM in children and adolescents is dramatically increasing. ${ }^{[861]}$ Onset of diabetes at a younger age is associated with longer disease exposure and increased risk for chronic complications. This young-onset T2DM essentially affects individuals of working age, further accentuating the adverse societal effects of the disease. Several studies have demonstrated that there has been a shift in the age ( $<30$ years) at onset of T2DM in India. ${ }^{[82-867]}$ The major predisposing diabetes risk factors in children and young adults include obesity, decrease in physical activity, family history, and sedentary lifestyle. In addition, other factors including prenatal factors (e. g., low birth weight, maternal under-nutrition), biological propensity to central obesity and insulin resistance, low lean mass, diabetes during pregnancy, impaired glucose tolerance, and urban stress are associated with high prevalence of T2DM in Indian children and young adults..$^{[44,45,868-872]}$

More recently, up to 1 in 3 new cases of diabetes mellitus in USA diagnosed in youth younger than 18 years is T2DM and is common among youth between 10 and 19 years of age. ${ }^{[861]}$

\section{Pathophysiology of type 2 diabetes mellitus in young versus adults}

The mechanism of development of T2DM in young people are similar to those in older patients; however, the speed of onset, severity, and interplay of reduced insulin sensitivity and defective insulin secretion might be different in patients who develop the disease at a younger age. ${ }^{[873]}$ Studies suggest that loss of $\beta$-cell function is accelerated in young-onset type 2 diabetes to $20-35 \%$ annual decline as compared to $7 \%$ decline in adults. ${ }^{[874]}$ This suggest that that T2DM in adolescents and children might have a more aggressive course along with loss of $\beta$-cell function as compared with adult later onset T2DM. ${ }^{[873]}$

\section{Screening and diagnosis}

The diagnosis criteria for children and adolescents are symptoms of diabetes mellitus such as polydipsia, polyuria, and unexplained weight loss plus casual glucose concentration $\geq 200 \mathrm{mg} / \mathrm{dL}(11.1 \mathrm{mmol} / \mathrm{L})$ in venous plasma, fasting glucose $\geq 126 \mathrm{mg} / \mathrm{dL}(7.0 \mathrm{mmol} / \mathrm{L})$ in venous or capillary plasma, or $2-\mathrm{h}$ glucose during OGTT $\geq 200 \mathrm{mg} / \mathrm{dL}(11.1 \mathrm{mmol} / \mathrm{L})$ in venous plasma or capillary whole blood or $\mathrm{HbAlc} \geq 6.5 \% \cdot{ }^{\left[{ }^{[875]}\right.}$ Children at substantial risk for the presence or the development of type 2 diabetes mellitus should be tested. Children and adolescents who are overweight (BMI $>90$ percentile) and have family history T2DM in first-or second-degree relative must be screened. Children with certain signs of insulin resistance or conditions associated with insulin resistance such acanthosis nigricans, hypertension, dyslipidemia, polycystic ovarian syndrome, must also be screened regularly. ${ }^{[876]}$

\section{Management of type 2 diabetes mellitus in children and adolescents}

The ideal goal of treatment is normalization of blood glucose values and $\mathrm{HbAlc}$. Weight control is essential for reaching treatment goals and are effective to treat type 2 diabetes mellitus in adolescents. Although lifestyle modification is the most commonly used intervention in adolescents with type 2 diabetes, less than $20 \%$ achieve or maintain adequate glycaemic control with lifestyle intervention alone ${ }^{[877]}$ Aerobic activity, alone or in combination with diet, can reduce systolic blood pressure, reduce total cholesterol, raise HDL cholesterol, and improve endothelial function in overweight children with T2DM. ${ }^{[878]}$ Metformin, is the most appropriate starting point for pharmacological treatment in children with T2DM. Results of the TODAY study suggest that monotherapy with metformin was associated with durable glycaemic control in children and adolescents. ${ }^{[879]}$ Bariatric surgery has emerged as a viable treatment option in young individuals with type 2 diabetes and evidence has shown that it is safe and effective in obese adolescents. ${ }^{[880]}$ However clinical data to support this are limited. 


\section{Fasting and Diabetes}

\section{RECOMMENDATIONS}

\section{Recommended Care}

- Fasting to be avoided in individuals with T2DM especially if they also have:

- Uncontrolled or unstable glycaemia history of recurrent diabetic ketoacidosis (DKA), significant macrovascular/microvascular complications or hypoglycaemic unawareness

- Individuals with T2DM should abstain from fasting if they are:

- On intensive insulin therapy or experience frequent hypoglycaemic episodes

- Non-adherent to advice on diet, drug regimens, and daily activities

- Antenatal or nursing or elderly or children

- Diabetic individuals who wish to fast must:

- Consult a physician prior to fasting

- Should be encouraged to participate in pre fast counselling/assessment to optimize monitoring/therapeutic strategies for optimal glycaemic control

- During fasting, patients with diabetes should always:

- Carry some sweets/glucose source to be used in case of hypoglycaemia

- Carry identification card displaying diabetic status and current medication

- Test blood glucose levels regularly (especially, if unwell during fasting)

- Treat promptly if glucose levels are deranged

- In case of dehydration or hypoglycaemic episode-end the fasting immediately

- Discuss with the physician regarding change in dose, and timing of insulin injections

- Hypoglycaemia may be prevented in four levels including primordial, primary, secondary and tertiary, using ASAP (Anticipate, Suspect, Act to treat, Prevent) strategy

- Metformin, incretin based therapies (sitagliptin, vildagliptin and liraglutide) and pioglitazone, glinides, AGIs, newer sulfonylureas like gliclazide MR and glimepiride are the preferable agents to be used during fasting that is spread over a number of days or weeks. In patients on insulin therapy analogues may be preferred over conventional insulins. Since prolonged fasting may involve significant reduction in fluid intake so SGLT-2 Inhibitors may be avoided.

- To minimize T2DM-related AEs during fasting, patient centred diabetes education, fasting nutrition plan with regular glucose monitoring and adjustment of treatment regimens is recommended.

\section{BACKGROUND}

Fasting is just not merely abstaining from food or 'starving', it is defined as the ability to meet the body's requirements for vital nutrients during either shortage or absence of food, by utilizing the body's energy reserves without jeopardising health and wellbeing. ${ }^{[608]}$ Periodic voluntary fasting, is a common religio-cultural practice adopted by individuals from various religions across the world for centuries as a crucial pathway of spiritual purification. ${ }^{[881,882]}$ Fasting or food abstinence, initiates metabolic and psychological changes and adaptations which need close monitoring, primarily in patients with derailed metabolism. Therefore, individuals with diabetes, or pre-diabetes must fast only after appropriate risk assessment and counselling with healthcare practitioners (HCPs) and religious leaders and make an informed decision. ${ }^{[608,609]}$ In diabetic individuals, insulin resistance and/or deficiency can lead to excessive glycogen breakdown and a surge in gluconeogenesis or ketogenesis leading to sudden hyperglycaemia, diabetic ketoacidosis, dehydration and thrombosis. ${ }^{[609]}$ Furthermore, in special populations like pregnant individuals, geriatric patients, individuals with comorbidities such as cardiac, renal or hepatic impairment, the risk of complications may increase if appropriate care is not taken. ${ }^{[92,690,712]}$ Therefore, a patient-centric approach with diet plan, and dose modification/omission with careful monitoring during fasting period may reduce the complications in patients with diabetes. Depending on the degree of abstinence from food, fasts may be classified as follows [Table 33].

\section{Religious fasts}

Although, religious fasts seldom exceed $24 \mathrm{~h}$, the variability of the duration of every phase may lead to different physiological responses to fasting particularly in patients with diabetes. ${ }^{[883]}$ Though several guidelines are available for different aspects of diabetes care, fasting in diabetes poses a unique challenge. ${ }^{[884,885]}$ Additionally, designing randomized controlled studies to address fasting related issues in patients with diabetes is particularly difficult. Therefore, understanding the physiology of fasting [Figure 18] and linking it to pathophysiology and clinical manifestation

\section{Table 33: Types of fast}

Complete fasting: Giving up food and water completely for a period Partial fasting: Eating less than you need to avoid hunger

Limiting the number of food items eaten

Giving up favourite foods 


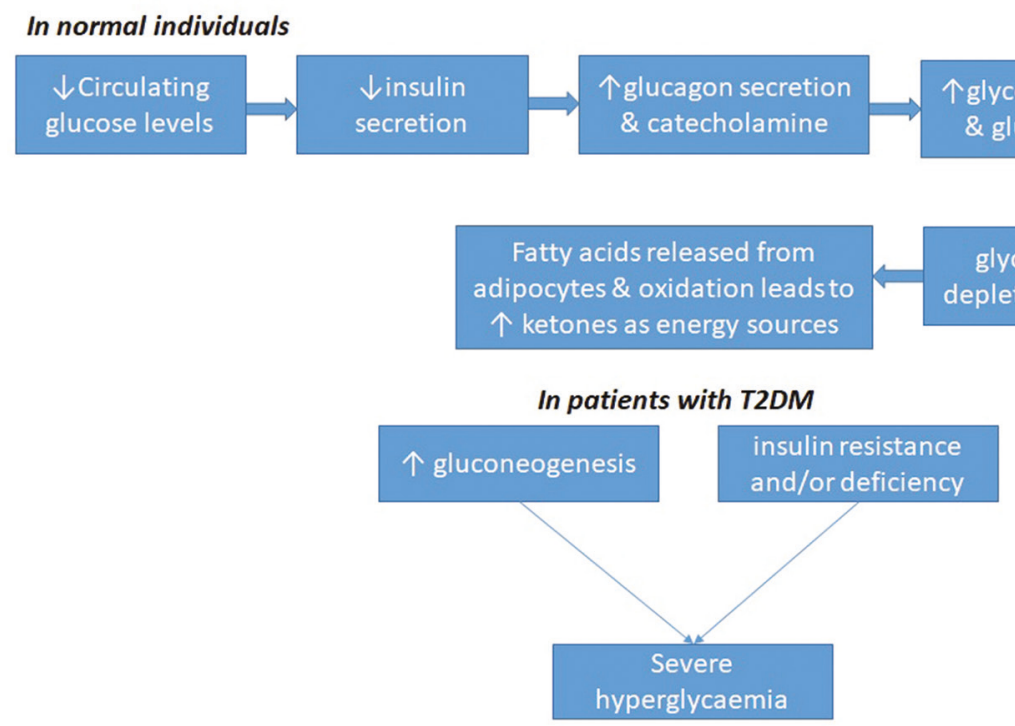

Figure 18: Physiology of glucose regulation in fasting. ${ }^{[883]}$ T2DM: Type 2 diabetes mellitus

\begin{tabular}{|c|c|c|}
\hline Pre-fast assessment & Pre-fast Counselling & Education \\
\hline $\begin{array}{l}\text { Complete annual review } \\
\text { of the patients' condition } \\
\text { \& clinical assessment } \\
\text { - Identification of } \\
\text { secondary complications } \\
\text { and co-morbidities } \\
\text { - Measurements of HbA1c, } \\
\text { blood pressure and lipids }\end{array}$ & $\begin{array}{l}\text { - Potential discomforts } \\
\text { and risks along with of } \\
\text { mitigating methods } \\
\text { - Duration of fast, } \\
\text { probable food intake } \\
\text { times } \\
\text { - Acceptance of types } \\
\text { foods, } \\
\text { - Possibility of } \\
\text { withdrawing fasting in } \\
\text { case of discomfort }\end{array}$ & $\begin{array}{l}\text { - Self-management and } \\
\text { monitoring practices } \\
\text { - Hypoglycaemia } \\
\text { awareness training } \\
\text { - Mitigations plans in case } \\
\text { of emergency adverse }\end{array}$ \\
\hline
\end{tabular}

Figure 19: Structured education program

\begin{tabular}{llll}
\hline Table 34: Factors to be modified & & & \\
\hline Fasting & Antidiabetic agents & Individual phenotype & Patient characteristics \\
\hline Duration of fast & Potential for hypoglycaemia & Risk of hypoglycaemia & Pregnancy \\
Restriction of fluids/solids: absolute/partial & Potential for dehydration & Risk of hypoglycaemia unawareness & Elderly \\
Frequency of fast (once weekly/once & Potential for gastrointestinal upset & Ability to self-monitor BG & Concomitant diseases \\
monthly/once yearly/others) & Duration of action & & Adolescent and children \\
\hline
\end{tabular}

BG: Blood glucose

of diabetes is required to design strategies for glycaemic management during fasting. ${ }^{[883]}$

The different religious fasts commonly observed in India that can have significant impact on metabolic and glycaemic health in diabetes are:

- Ramadan fasting: It is a principle ritual followed by Muslims during the sacred month of Ramadan (the ninth lunar month of Islamic/Hijri calendar). [886] During this month, all healthy adult Muslims abstain from food, drinks and medication from dawn to dusk (sunset). Believers usually eat two meals, one before dawn (Suhur) and one after sunset (Iftar). Hypoglycaemia and dehydration are major complications associated with fasting though hyperglycaemia may occur, due to over indulgence in food during the two main meals of Suhur and Iftar. $^{[886,887]}$ Therefore, pre fast risk stratification, followed by a treatment tailored to individual needs appears to be the best management strategy. In addition, structured education enables patients to self-manage their condition better. ${ }^{[885-887]}$ 
- Hindu fasts: Though not mandatory, most Hindus observe day-long and week-long fasts. KarvaChauth, Guru Purnima, Ekadashi, Makar Sakranti and Holi Ashtami are some of the annual, monthly and weekly fasts observed as part of various vows. During Navratri, which occurs twice a year, Hindus observe fast for 9 days usually from dawn to moonrise/star-rise. The day-long nature of Hindu fasts however makes it distinct from the month-long fasts of Ramadan and Buddhist Lent. Unlike Islam, there are no universal rules laid down for Hindu fasts, and therefore data on metabolic effect of these fasts are scanty thus far. ${ }^{[888,889]}$

- Jain fasts: During the pious month of Paryushana (eight days for the Shwetamber sect, and ten days for the Digamber sect), Jains usually fast from dusk to dawn unlike Hindu fasting which extends from dawn to moonrise. $^{[822]}$

\section{Considerations}

Based on the following factors the glucose lowering therapy/ strategy during fasting period may be modified/altered [Table 34].

\section{Rationale and Evidence}

Complete abstinence from food and drink between sunrise and sunset can lead to disruption of homoeostasis. Since the majority of diabetic individuals are asymptomatic, they are unaware of the potential deleterious effects of diabetes, particularly during religious fasts. Additionally, conditions of complete abstinence from food and/or water during religious fast leads to skipping medications, culminating in to derailed metabolism and worsening of their condition.

- An observational study in Muslim patients with diabetes fasting during Ramadan reports that 59\% patients had substantial knowledge of diabetes, 37\% patients did not monitor their blood glucose levels in the previous Ramadan and $47 \%$ had hypoglycaemic episodes. ${ }^{[890]}$

- In a prospective clinical study conducted in Iran the glycaemic control deteriorated significantly among T2DM patients who opted to fast during Ramadan and however the, HbA1c levels reduced significantly following the month after Ramadan. ${ }^{[891]}$

\section{Recommendations on management of diabetes during fasting}

Concerned physicians and the HCPs may play a critical role in educating the patients with diabetes during fasting [Figure 19]. Patients and their families should be included in a structured diabetes educational programme, which gives information on risk quantification, physical activity, glucose monitoring, diet, hypoglycaemia, dosage and timing of medications, and identification of the symptoms of complications. ${ }^{[892]}$
- Implementation of the Ramadan Education and Awareness in Diabetes (READ) programme led to significantly lower weight gain $(\mathrm{p}<0.001)$ and hypoglycaemic episodes $(\mathrm{p}<0.001)$ with reduced risk of acute complications compared to those who were not educated during fasting. ${ }^{[358]}$

- Self-monitoring of blood glucose (SMBG) should be considered as an important tool that helps both patients and physicians to practice safe decision making regarding drug dosage and other aspects of management. ${ }^{[893]}$

- $\quad$ Patients who received individualized education are more likely to modify their diabetes treatment plan during Ramadan, perform self-monitoring of blood glucose at least twice daily during Ramadan and to have improved knowledge about hypoglycaemic signs and symptoms as compared to patients who followed the standard diabetes management protocol. ${ }^{[894]}$

\section{Lifestyle modification and nutrition}

- $\quad$ Fasting or healthy abstinence from food, is a form of lifestyle modification for T2DM patients and if utilized appropriately, may result in several health benefits to these patients. ${ }^{[895]}$

- $\quad$ Pre-fasting diet should include slow release foods and patients with T2DM should not indulge in over-eating in the post-fasting period in order to avoid postprandial hyperglycaemia. ${ }^{[85]}$ Therefore, complex carbohydrates like whole grains, potato, berries, citrus fruits, apple, nuts and legumes at pre-fasting, and simple carbohydrates like bread, cereals, rice, and pasta at post-fasting may be more appropriate to reduce complications. ${ }^{[886]}$

- During prolonged fasting periods like Ramadan or Navaratri, physical activity should be restricted. While routine exercise can be continued, elective moderate to highly vigorous exercise should be rescheduled. ${ }^{[888]}$

\section{Pharmacological management}

- Individualized or bespoke treatment choices must be made for oral agents during fasting period. ${ }^{[896]}$ Antidiabetic agents that improve the insulin sensitivity must be chosen as the risk of hypoglycaemia is significantly lower. ${ }^{[885]}$

- $\quad$ Biguanides (Metformin) are generally considered safe in patients with diabetes during fasts due to minimal incidences of hypoglycaemia, however, once daily dosing need to be adjusted or modified to avoid complications. ${ }^{[897]}$ Slow-release formulations of metformin must be taken once daily following the sunset meal. ${ }^{[898]}$

- Sulphonylureas (new generation: gliclazide MR and glimepiride) should be preferred over older, long acting sulfonylureas like glibenclamide and chlorpropamide during Ramadan fasting, as they are relatively more safe and economical. ${ }^{[171,884,887,899]}$ Glibenclamide may be associated with a higher risk of hypoglycaemia than other second generation sulfonylureas like gliclazide, glipizide, and glimepiride. ${ }^{[884]}$ Therefore use of sulfonylurea should be individualized. 
- Thiazolidinedione (pioglitazone and rosiglitazone) are generally regarded as safe and in patients who fast during Ramadan, however, it may lead to an increase in body weight. ${ }^{[900]}$

- Incretin based treatments may maintain adequate glycaemic control in a glucose-dependent manner, thus providing a safe alternative therapeutic option during Ramadan $^{[885]}$

- Vildagliptin was found to be effective, safe, and well tolerated in T2DM patients fasting during Ramadan, with a consistently low incidence of hypoglycaemia across studies, accompanied by good glycaemic and weight control. ${ }^{[260]}$

- Switching anti-hyperglycaemic treatment to sitagliptin from a sulfonylurea reduced the risk of symptomatic hypoglycaemia by approximately $50 \%$ in patients who fasted during Ramadan. ${ }^{\text {[901] }}$

- In Treat 4 Ramadan trial, liraglutide compared with sulfonylurea was well tolerated with more patients achieving target $\mathrm{HbAlc}$, lose or maintain weight with no severe hypoglycaemia and with high level of treatment satisfaction. ${ }^{\text {902] }}$

- Contrary to the Treat 4 Ramadan trial, no significant difference between liraglutide and sulfonylureas in terms of severe hypoglycaemia. However, significant, weight loss and HbAlc reduction $(\mathrm{p}<0.0001)$ were observed in liraglutide group suggesting that liraglutide may be considered an effective therapy in combination with metformin during Ramadan. ${ }^{[903]}$

- Sodium-glucose cotransporter 2 inhibitors may be used during fasting, in view of their low risk of hypoglycaemia. However, the potential risk of dehydration must be considered.

- Treatment with dapagliflozin was associated with fewer incidences of hypoglycaemia than sulfonylureas $(\mathrm{p}=0.002) \cdot{ }^{[004]}$

- A recent survey report conducted in physicians highlights that SGLT2 inhibitors are safe and effective for T2DM management during Ramadan and (92.2\%) physicians suggested prescribing SGLT2 inhibitor with the first evening meal (Iftar). ${ }^{[05]}$

- Use of a rapid acting insulin analogue instead of regular human insulin before meals in patients with T2DM who fast during Ramadan was associated with less hypoglycaemia and less PPG excursions. ${ }^{[906]}$

Detailed information on categories of risk in patients with T2DM who fast during Ramadan can be found in Annexure 8.

Detailed information on recommended changes in treatment regimens of OADs and insulin in patients with T2DM who fast during Ramadan and other religious fasts can be found from in the Annexure 9.

\section{EdUCATION}

\section{ReCOMMENDATIONS}

\section{Recommended Care}

- A patient-centred, structured diabetes self-management education (DSME) is an integral part of the care of all people with T2DM.

- The diabetes self-management education and support (DSMES) program should be conducted at four critical times: at diagnosis, annually, when complicating factors arise, and when transitions in care occur.

- Education programs can be conducted by medical professionals, certified diabetes educators who are quality assured to provide education (Certified Diabetes Educators) either in groups or individual settings. If needed, any family member, friend or a caretaker should be involved.

- The education program should focus more on rural or poorly educated patients as they have less knowledge and awareness regarding diabetes. Different education material may be needed for such patients.

- Every primary care unit should facilitate the training of at least one of their health professionals to become a diabetes educator.

- Diabetes education should be focused towards the assessment of change in behaviour of patients and promote self-management in patients with T2DM.

- Ensure that DSME programs are accessible to all patients and designed to address cultural needs, ethnicity, psychosocial concerns, medical history, family support, literacy, disability issues and financial status.

- Use techniques of active learning (engagement in the process of learning and with content related to personal experiences), adapted to personal choices and learning styles.

- Use modern communications technologies to advance the methods of delivery of diabetes education such as one-on-one and group sessions and use of social media.

- Provide on-going diabetes self-management support, creation of self-help groups 


\section{BACKGROUND}

Diabetes self-management education (DSME) is a key component of management of T2DM, facilitating the knowledge and skills required to improve self-care practices to prevent or delay progression and development of diabetes. ${ }^{[222,907,908]}$ Numerous studies report that DSME is associated with improved metabolic control, reduced glycaemic levels, fewer complications, and improved quality of life (QoL). ${ }^{[359,907,909]}$ DSME program is aimed to improve diabetic knowledge and empower people to make informed choices to self-manage their condition more effectively. ${ }^{[702,909]}$ It is guided by evidence-based standards while incorporating needs, goals and life-experiences of the patients with diabetes. ${ }^{[910]}$ The 2019 Standards of Medical Care in Diabetes recommends that people living with diabetes should be actively engaged in education, self-management, and treatment planning with their health care team, including the collaborative development of an individualized eating plan. ${ }^{[911]}$ The 2019 consensus report by ADA and EASD on management of hyperglycaemia in T2DM recommends that all people with T2DM should be offered access to ongoing DSMES (Diabetes self-management education and support) programs. ${ }^{[90]}$

India represents a country with diversity in social, economic, cultural, and educational patterns. Majority of Indian population resides in rural areas, where there is lack of knowledge and awareness on diabetes than urban population. ${ }^{\left[{ }^{[912]}\right.}$ No or low literacy in India is the key deterrent to poor level of awareness of diabetes. ${ }^{[82,913]}$ Effective patient education is an essential tool in resource poor settings like India, where prevalence of T2DM has escalated to epidemic proportions over past 2 decades.

\section{Considerations}

The panel endorsed the IDF recommendations on education as such. However, evidence from India together with local factors such as cost, literacy, malnutrition, body weight, and BMI were reviewed in the Indian context and are reflected in the recommendations.

\section{Rational and Evidence}

\section{Educational programs and their outcomes}

- In management of T2DM patients, structured diabetes care program (Freedom $365^{*}$ ) of ongoing diabetes education on diet and lifestyle correction, biochemical investigations, clinical monitoring, and treatment at regular intervals was associated with better clinical outcomes compared to routine medical care. The program played a pivotal role in improving the patient's quality of care by overcoming clinical inertia, improving adherence to therapy, while preventing the occurrence/progression of diabetes associated complications. ${ }^{[914]}$

- Organized diabetes education, that involves improving knowledge on better control of disease symptoms, disease regimens, and risks in practice was found to have a positive impact on lifestyle changes, self-control abilities, and improving the QoL in T2DM patients. ${ }^{[915]}$

- A recent systematic review including 118 unique interventions reported that DSME was associated with a statistically significant mean reduction in $\mathrm{HbAlc}$ levels (-0.74 for intervention and-0.17 for control groups). ${ }^{[007]}$

- A case control study conducted in the department of medicine of a tertiary care teaching hospital in north-west India demonstrated that effective health education improves knowledge, attitude, and practices leading to better glycaemic control that can slow down the progression of diabetes and prevent downstream complications. ${ }^{[451]}$

- To minimize the increasing burden of NCDs, ministry of health and family welfare, Government of India, has launched the National Programme on Prevention and Control of Diabetes, Cardiovascular diseases and Stroke (NPDCS) on 8th January 2008 with several objectives including health promotion and health education for the community. ${ }^{[916]}$

- Besides diabetes, educational intervention was also successful in reducing some of the obesity parameters and improving dietary patterns in individuals with prediabetes and diabetes. Initiation of primary prevention strategies through education right from elementary schools could reduce IFG by $17 \%$ suggesting such interventions may delay T2DM or even change the course of disease for improved outcomes among vulnerable population groups. ${ }^{[75]}$

- Awareness about early detection and treatment of hyperglycaemia in pregnancy is also important, as it will not only offer better fetal outcome, but a good glycaemic control during pregnancy will provide better intrauterine metabolic environment, which may help to prevent the development of diabetes, obesity, and metabolic syndrome in these offspring of diabetic mother in their later life. ${ }^{[18]}$

- India held the world's first national GDM (NGDM) Awareness Day on March 10, 2019 for raising awareness about the link between maternal health and diabetes nationwide, and invited pregnant women to hospitals and clinics for free screening. The program included training for healthcare professionals, press conferences, awareness raising events, seminars for women's group, and widespread screening. ${ }^{[3]}$

\section{Knowledge and awareness}

- The ICMR-INDIAB study reported that the awareness of diabetes in urban India was significantly higher than rural residents $(58.4 \%$ vs $36.8 \%, \mathrm{p}<0.001)$. Furthermore, participants from Tamil Nadu had the highest (31.7) and Jharkhand the lowest (16.3) knowledge score, and among self-reported patients with diabetes, Maharashtra had the highest (70.1) and Tamil Nadu, the lowest score (56.5). ${ }^{[912]}$

- Similarly another ICMR-INDIAB study including 14,277 
participants revealed that only 480 patients were with selfreported diabetes (254 urban and 226 rural) and the level of glycaemic control among patients with self-reported diabetes in India was poor. ${ }^{[36]}$

- A population-based study from a south Indian state reported that among 6211 participants, good knowledge and positive attitude were observed in $3457(55.6 \%)$ and $3280(52.8 \%)$ people, respectively. Furthermore, literacy had a significant association with good knowledge, attitude, and practice in general and T2DM population. Overall, women had significantly better knowledge $(\mathrm{p}<0.001) .{ }^{[917]}$

- A recent study from east Delhi, India reported that selflearning module (SLM) was significantly associated with increasing knowledge on aspects of effect of diabetes on foot $(p<0.05)$, foot care and its steps $(p<0.05)$ than control group in T2DM patients. ${ }^{[918]}$

- Though general practitioners in India are aware and updated about symptoms and screening of T2DM, there is dearth of effective approaches towards screening and treatment of complications. Most patients are usually not advised on non-pharmacological measures and diabetes education, while interpretation of test results for screening of disease and its complications appear to be a major flaw in general practice. ${ }^{[51]}$

- Evidence from several studies determining the level of knowledge and awareness on diabetes across India suggests that most of the patients had poor knowledge and awareness about their condition. ${ }^{[12,919-925]}$ Low socioeconomic status, old age, cultural factors, lack of access to healthcare, family history of diabetes, and importantly low literacy levels were the major predictors of poor glycaemic control among patients with T2DM.

- A cross-sectional, questionnaire-based survey was conducted in 100 patients attending the diabetic unit of a tertiary care teaching hospital in central India. Majority of patients were found to be aware about hypoglycaemic symptoms and its treatment and development of complications. Regular check-up was done by $70 \%$ of patients, while $73 \%$ were adhered to treatment. ${ }^{[926]}$

- A cross-sectional survey was carried out among participants aged $\geq 18$ years, visiting tertiary care eye institute in north India to assess awareness of people about various aspects of diabetes. Of 530 participants interviewed, only $40(25.6 \%)$ diabetic, and 45 (13.8\%) non-diabetic participants were aware about diabetic retinopathy. Their knowledge about its risk factors, complications, prevention, and management was poor. ${ }^{[227]}$

- In a study conducted on 400 diabetic patients (out-patient or admitted), awareness of diabetic nephropathy was marginally higher in patients staying in urban areas (vs rural areas, $\mathrm{p}=0.120$ ) and among the literate (vs uneducated, $\mathrm{p}=0.567$ ) patients. Awareness of diabetic nephropathy was higher in older patients $(\mathrm{p}=0.004)$ and in patients with chronic diabetes $(p<0.0001)$, controlled diabetes $(p=0.026)$, and diabetic nephropathy $(\mathrm{p}<0.0001) .{ }^{[928]}$

\section{Challenges in diabetes management in India ${ }^{\text {[02,929-931] }}$}

- The awareness about the disease and its complications is less than satisfactory.

- There is lack of knowledge, attitude, and practice studies to determine the gaps in knowledge among people living with diabetes and physicians in the areas of individual diabetes care in India. Physician-related issues including inadequate knowledge, delay in clinical response, poor control need to be addressed through diabetes education.

- Lack of knowledge among people living with diabetes is a significant barrier to their ability to self-manage the disease. Hence, there is an imperative need for more structured diabetes education programs in India.

- Lack of strong referral system to provide quality care i. e. early diagnosis, prevention and control of chronic complications in diabetes. Specialist referral for diabetes management is a major challenge in remote and rural primary care facilities, often lacking trained diabetes specialists. ${ }^{[932]}$

- Indian studies have also shown that barriers to insulin therapy are due to lack of awareness, causing wrong perception, and false beliefs. People with ongoing insulin therapy had better understanding and acceptability towards insulin therapy than those not on insulin; besides intensification remains a challenge in these patients. ${ }^{[933]}$

- Implementing efficacious health service intervention like patient education in a real-world resource-constrained setting is challenging and may not prove effective in improving patient outcomes. Therefore, interventions need to consider patients' and healthcare providers' experiences and perceptions and how macro-level policies translate into practice within local health systems. ${ }^{[934]}$

- In India, counselling of young unmarried women with diabetes eroding familial support and marital prospects is particularly challenging due to disease-related stigma. ${ }^{[935]}$

\section{Assessing the need for evidence-based education ${ }^{[936-940]}$}

- Diabetes educator (nurse, dietician, social worker, or qualified diabetes educator) can play a major role in raising diabetes awareness and optimal diabetes care.

- Continuous medical education and periodic trainings are needed to help health professionals integrate new knowledge and transform old practices.

- Need to assess the impact of existing education and training programs in diabetes.

- Investment must be made to ensure that specialized diabetes education is accessible to healthcare personnel and people with diabetes.

- General practitioners and physicians should be periodically updated on recent guidelines on diagnosis, treatment as well as management goals.

- Key aim of diabetes education is to change behaviour of people and promote self-management.

- Steps to improve awareness in diabetes care in India:

- Physician and family physician education 


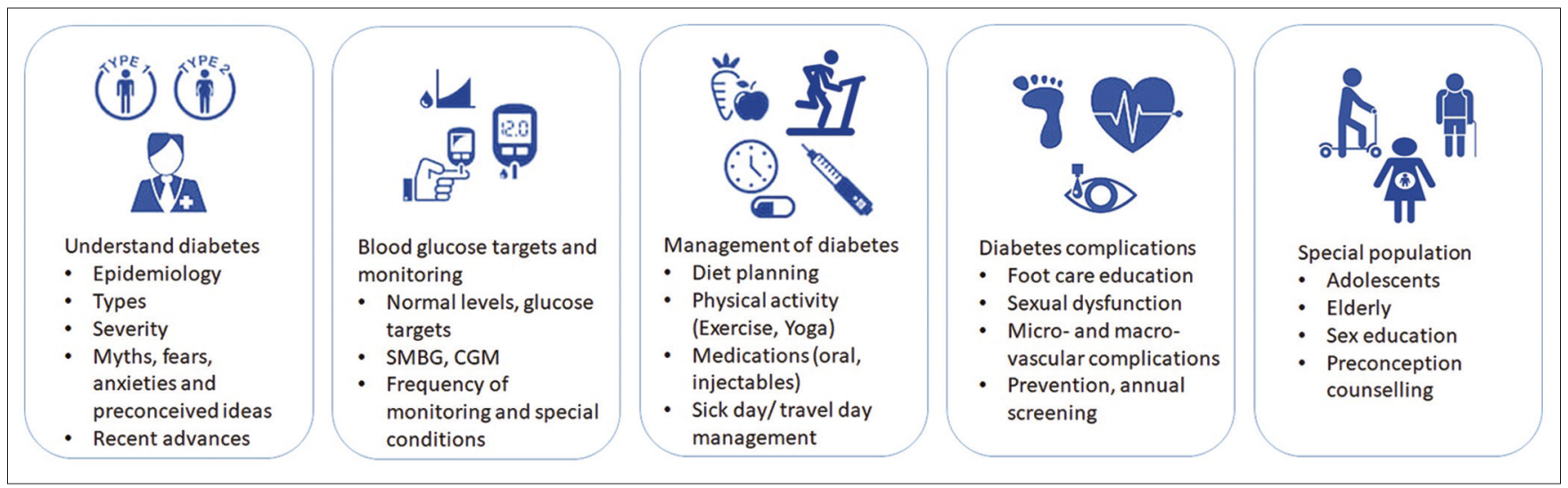

Figure 20: Components of diabetic education. CGM: Continuous glucose monitoring; SMBG: Self-monitoring of blood glucose

- Need for continuing medical education

- Education for patients with diabetes

- Diabetes education programs in India.

- Study has shown that pharmacist may also be involved with clinicians as a part of collaborative diabetes care and has documented positive clinical, humanistic, and economic outcomes, which emphasized the value of multidisciplinary collaborative care for Asian diabetes patients and supported the effectiveness of this approach in managing chronic diseases. ${ }^{[940]}$

- Counselling is the most important strategy capable of bringing about sustained lifestyle changes.

\section{IMPLEMENTATION}

- Major components of implementing these recommendations are the recruitment of personnel and their training in the principles of both diabetes education and behaviour change strategies. The staff are required to develop theoretically-based, patient-centred, and ongoing and follow-up education programs for people with diabetes. Educational strategies and materials aligned with the needs and culture of the community served with attention to health literacy are necessary. Institutional support at the practice, community, and health care system levels is critically important [Figure 20].

- Diabetes discrimination at education institutes and work places is often the result of a lack of knowledge about diabetes. Since diabetes is usually a "hidden" disability, many patients do not understand the gravity of the condition and what it is like to have diabetes. By educating such authorities about diabetes and their needs and abilities, they may be able to get fair treatment.

- Mass awareness campaign through various print and electronic media will also be an effective model of education.

\title{
Psychosocial Issues
}

\section{ReCOMmEndations}

\author{
Recommended Care \\ Approach to care \\ - Diabetes management should be carried out within a framework of informed and shared decision making, following the philosophy of responsible \\ patient-centred care. \\ - Psychosocial care should be provided to all individuals with T2DM using a collaborative, patient-centred care approach with referral to mental health \\ care professional where needed. \\ - Family members and other close ones in the management of diabetes must be involved \\ - Self-disclosure of diabetes, as opposed to maintenance of confidentiality, should be decided on a case-to case basis, keeping the sociocultural \\ environment in mind \\ - HCPs should take care of their own psychosocial health, as compassion fatigue is a common professional hazard. \\ Assessment \\ - Periodic assessment of psychosocial well-being should be done using questionnaires or validated tools (e. g. WHO-5, PAID, Whooley's 2-item \\ questionnaire)
}




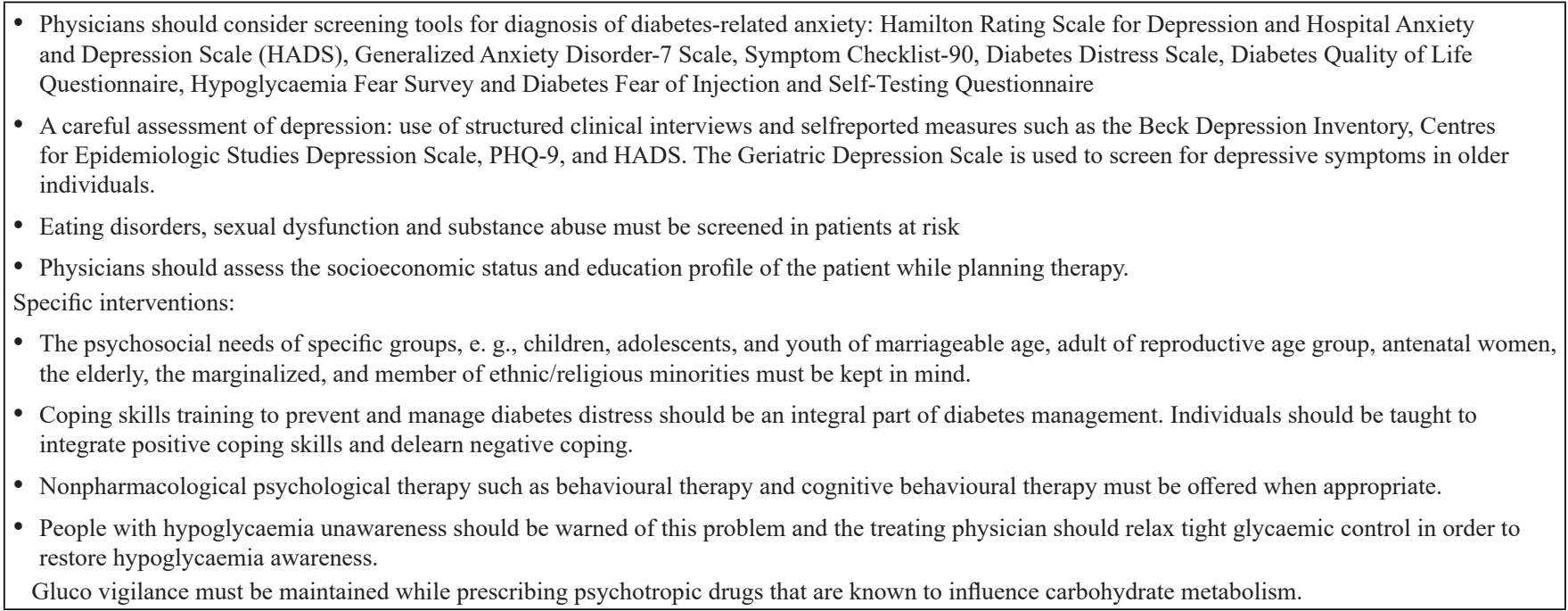

\section{Limited Care}

- Be alert to signs of cognitive, emotional, behavioural and/or social problems which may negatively impact quality of life and complicate self-care, particularly where diabetes outcomes are sub-optimal.

- Refer to mental health specialist advice according to local availability of such professionals.

\section{BACKGROUND}

Complex environmental, social, behavioural, and emotional factors, together known as psychosocial factors, play a crucial role in optimum diabetes management and achieving satisfactory medical outcomes. The daily demands of the disease course and management interrupts the psychological well-being of people with diabetes. The prevalence of comorbid psychosocial problems is greater in patients with diabetes than in the general population. ${ }^{[941]}$ The psychological and social issues such as stress, anxiety, depression, eating disorders, cognitive dysfunction, or other psychological disorders are associated with poor self-care, increased mortality, functional impairment, increased healthcare cost, loss of productivity, and reduced quality of life. ${ }^{[942-945]}$ Patient's psychosocial conditions have significant impact on the overall outcomes of diabetic care process. ${ }^{[946-948]}$

Euthymia is a target, as well as a tool, for diabetes management. ${ }^{[949,950]}$ Psychosocial well-being comprehends both physical and mental health, and is integral to diabetescare and self-management. The ADA and the American Association of Diabetes Educators (AADE) have focussed on the role of diabetes self-management education and support (DSMES) on improving psychosocial benefits, including the reduction of depression. ${ }^{[951-953]}$ In addition, integrating mental health services in diabetes management can help with effective coping strategies. The IDF 2018 guideline has suggested the inclusion of a mental health professional in the multidisciplinary team and highlighted the need for counselling a patient in the setting of on-going diabetes education and care. ${ }^{[954]}$ The AAACE has recommended, cultural and faith-based aspects of therapy during counselling. ${ }^{[955]}$

In India, heterogeneity in linguistic, cultural, religious, socioeconomic, educational, regional, and familial factors affects the clinical progression, treatment and outcome of diabetes management. While family and community support medically-impaired persons as a part of our ethos, societal insensitivity often exhibits itself, as culinary cruelty in many ways for example: Indian patients with T2DM showed a significantly higher perception of burden of social and personal distress associated with the disease, and have been reported to have one of the lowest levels of psychological well-being based on the World Health Organization-5 (WHO-5) Wellbeing Index.$^{[952,956]}$ These challenges and strengths warrant thedevelopment of India-specific recommendations for psychosocial management of diabetes, sensitive to and appropriate for, the Indian context. ${ }^{[957]}$

\section{Considerations}

Diabetes care and self-management is likely to be affected in presence of a mental health disorder that notifies patient's psychosocial condition. Detection of such disorders in relatively brief consultations with diabetes professionals is challenging. There is a need for some basic training to diabetes professionals in management of psychosocial issues, and for 


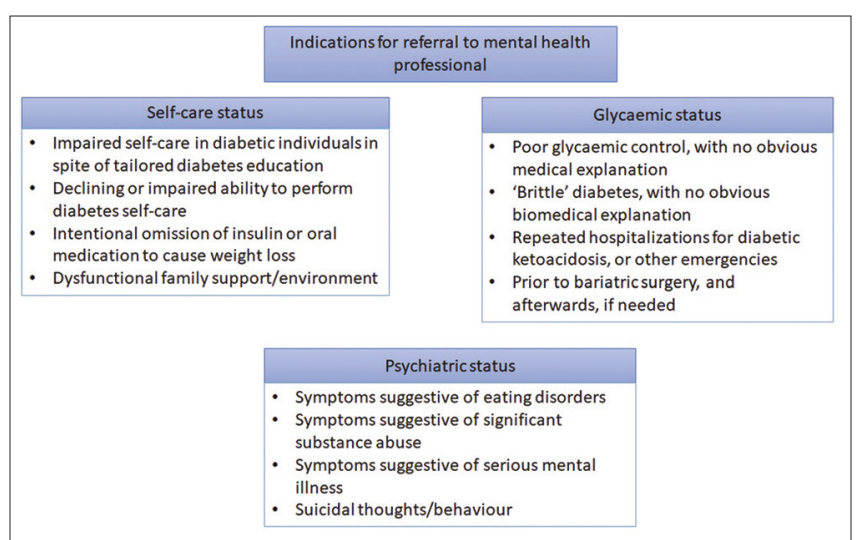

Figure 21: Indications to consider for referral to mental health professional

appropriate referral approach to mental health professionals with a knowledge of diabetes, especially for seriously affected patients.

\section{Rational and Evidence \\ Challenge}

- Being diagnosed with diabetes imposes a life-long psychological burden on the patient and his/her family. Prevalence of clinically significant depression, anxiety, eating behaviour disorder are considerably more common in patients with diabetes than in those without the disease. ${ }^{[958-960]}$

- The findings from a systematic review and metaanalysis conducted on 248 observational studies demonstrated that almost one in four adults with T2DM experienced depression; while depression was more common in patients with $<65$ years of age compared with elderly. ${ }^{[961]}$

- Poor psychological functioning can seriously interfere with daily diabetes self-management, with subsequent poor medical outcomes and high costs. ${ }^{[962,963]}$

\section{Solution}

- Collaborative care interventions and a team approach for diabetes management have demonstrated efficacy in selfmanagement with improved psychosocial outcomes. ${ }^{[964,965]}$

- A systematic review and meta-analysis has shown that, overall, psychological interventions are effective in improving glycaemic control in T2DM. ${ }^{[966]}$

- A randomized-controlled study showed that web-based guided self-help centred on cognitive behaviour therapy for people with diabetes with mild-to-moderately severe depression is effective. ${ }^{[967]}$

- Psychological counselling can contribute to improved adherence and psychological outcomes in people with diabetes [Figure 21]. ${ }^{[968]}$

\section{Implementation}

- Major components of implementing these recommendations is the involvement of HCPs and their training on principles of both diabetes education and psychosocial interventions.

- HCPs are required to develop collaborative, patientcentred medical care strategy for all patients with diabetes to improve health outcomes and quality of life.

- HCPs must be trained in applying psychological assessment tools and monitoring procedures, for diagnosis and periodic evaluation.

- Collaboration with mental health specialists who have knowledge in diabetes can help extend the education and training of other mental health specialists in relation to diabetes.

\section{Complementary and Alternate Therapy}

\section{RECOMMENDATIONS}

\section{Recommended Care}

- Physicians, diabetologists and registered dieticians who are trained to practice modern system of medicine are advised not to prescribe complementary and alternative medicine to treat T2DM.

- Complementary and alternative medicine therapies should not replace conventional modalities of T2DM management. 


\section{BACKGROUND}

The chronic nature of T2DM and its course of treatment often intimidates patients with the possibility of a lifetime treatment with conventional allopathic medications. Complementary medicine refers to interventions used in conjunction with conventional pharmacological treatment while alternative medicine are treatments that replace modern medicines. ${ }^{\left[{ }^{[69]}\right.}$ Complementary and alternative medicine (CAM) comprise mind-body practices (e. g. yoga, pranayama, meditation, tai chi, massage therapy, acupuncture etc.), natural remedies (usually herbal dietary supplements) or treatments from a traditional system of medicine. ${ }^{[755,969]}$ The use of CAM in India has been quite popular especially with the use of traditional Indian practices-Ayurveda, Yoga, Naturopathy, Unani, Siddha and Homeopathy. Studies exploring the use of CAM in India reported a prevalence of $68 \%$ in Uttar Pradesh, $63 \%$ in Maharashtra and almost 39\% in rural Kerala among T2DM patients. ${ }^{[970-972]}$ It was also observed that the level of physician consultation among CAM users was generally low $^{[970,972]}$ [Table 35].

Medicinal herbs with potential anti-diabetic effects have been extensively studied in India. Most of these are indigenous to the Indian diet and are consumed as a part of everyday meals. However, studies investigating the glycemic effects of these herbs (methi, vijaysar, gurmar, neem, amla, ghritkumari, turmeric, black pepper, ginger, cinnamon, dates, onion, and karela) have shown inconsistent findings ${ }^{[753,755,973-976]}$ Supplementation with chromium, alphalipolic acid, omega- 3 fatty acids, magnesium, and zinc have also shown minimal antidiabetic effects, however, the long-term use and interactions between herbs and medications are known to cause some serious side effects. ${ }^{[977]}$ The safety of these agents for T2DM is greatly challenged due to the lack of stringent regulations for their approval, manufacturing and marketing. ${ }^{[978,979]}$ Also there is a serious lack of high-level evidence from adequately powered randomized controlled studies to validate the clinical efficacy and safety of these preparations. ${ }^{[980]}$ Several Ayurveda and herbal Indian medicines have been found to be contaminated with heavy metals (lead, arsenic and mercury) and known to have serious health consequences. ${ }^{[69,981]}$ There is a general trend of increased consumption of herbal remedies in patients - alone or concomitantly with prescribed medicine. A study conducted in patients with T2DM in a tertiary care teaching hospital of North India reported that $45 \%$ patients were taking herbal remedies along with the prescribed drugs for diabetes and a majority (36/45) were taking these on their own or after advice from family members or friends; only $5 \%$ patients consulted an herbal specialist/ayurvedic doctor before starting these and $98 \%$ of cases the treating physicians were unaware of this fact. ${ }^{[982]}$ However, yoga, pranayama, meditation, acupuncture, massage therapy, aromatherapy and many relaxation techniques are being practiced in India, for prevention and management of diabetes. ${ }^{[555,976,983]}$

\begin{tabular}{ll}
\hline Table 35: Complementary and alternative medicine \\
\hline Mind-body practices & Traditional system of medicine \\
\hline Yoga & Ayurveda \\
Pranayama & Yoga \\
Meditation & Naturopathy \\
Tai chi & Unani \\
Massage therapy & Siddha \\
Acupuncture & Homeopathy \\
\hline
\end{tabular}

\section{Yoga}

Mind-body techniques utilize the influence of mind on the physical body and in the recent times, these have been popularly adapted into mainstream management of T2DM. Yoga, which is native to India, involves coordinated physical body movements, meditation, breathing exercises and chanting. The intensity of yoga activities can be adjusted based on the patients' needs and capabilities and used more effectively as a form of exercise forpatients with diabetes. However, there is inadequate clinical evidence showing the benefits of yoga for glycemic control and therefore, it cannot substitute physical activity completely. ${ }^{[969,984,985]}$ A randomized controlled study conducted in North India showed a significant improvement in the quality of life in the intervention group practicing the comprehensive yogic breathing program in addition to standard treatment of diabetes. Additionally, a nonsignificant trend towards improvement in glycaemic control was observed. ${ }^{[986]}$ In a prospective case-control study conducted in 30 men with T2DM on diabetic diet and oral hypoglycaemic agents, initiation of yoga as an adjuvant treatment was associated with significant improvement in fasting and post meal glucose levels. ${ }^{[555]}$ Since the risks associated with practicing yoga and meditation are minimal and it is known to have a favourable impact on sleep and overall well-being, it should be encouraged among patients with T2DM as a part of lifestyle management. ${ }^{[984,985]}$ Refer to the "Lifestyle changes and medical nutrition therapy section for additional information.

\section{Acknowledgements}

The authors thank Shweta Pitre, CMPP for writing support and Sangita Patil, PhD for editorial support (SIRO Clinpharm Pvt. Ltd, Maharashtra, India).This RSSDI clinical practice recommendations for management of type 2 diabetes mellitus is a revision of RSSDI clinical practice guideline for management of type 2 diabetes mellitus published in 2017.

\section{Financial support and sponsorship}

Nil.

\section{Conflicts of interest}

There are no conflicts of interest. 


\section{RefEREnCES}

1. American Diabetes Association. 2. Classification and diagnosis of diabetes: Standards of medical care in diabetes-2019. Diabetes Care 2019;42:S13-28.

2. Nguyen KA, Peer N, de Villiers A, Mukasa B, Matsha TE, Mills EJ, et al. Glycated haemoglobin threshold for dysglycaemia screening, and application to metabolic syndrome diagnosis in HIV-infected Africans. PLoS One 2019;14:e0211483.

3. International Expert Committee. International Expert Committee report on the role of the A1C assay in the diagnosis of diabetes. Diabetes Care 2009;32:1327-34.

4. Hasslacher C, Kulozik F, Platten I. Glycated albumin and HbA1c as predictors of mortality and vascular complications in type 2 diabetes patients with normal and moderately impaired renal function: 5-year results from a 380 patient cohort. J Diabetes Res Clin Metab 2014;3:9.

5. Lauritzen T, Sandbaek A, Skriver MV, Borch-Johnsen K. HbA1c and cardiovascular risk score identify people who may benefit from preventive interventions: A 7 year follow-up of a high-risk screening programme for diabetes in primary care (ADDITION), Denmark. Diabetologia 2011;54:1318-26.

6. Lim WY, Ma S, Heng D, Tai ES, Khoo CM, Loh TP. Screening for diabetes with $\mathrm{HbA} 1 \mathrm{c}$ : Test performance of $\mathrm{HbA1c}$ compared to fasting plasma glucose among Chinese, Malay and Indian community residents in Singapore. Sci Rep 2018;8:12419.

7. Guo F, Moellering DR, Garvey WT. Use of HbA1c for diagnoses of diabetes and prediabetes: Comparison with diagnoses based on fasting and 2-hr glucose values and effects of gender, race, and age. Metab Syndr Relat Disord 2014;12:258-68.

8. Radhakrishna P, Vinod KV, Sujiv A, Swaminathan RP. Comparison of hemoglobin A1c with fasting and 2-h plasma glucose tests for diagnosis of diabetes and prediabetes among high-risk South Indians. Indian J Endocrinol Metab 2018;22:50-6.

9. Prakaschandra R, Naidoo DP. Fasting plasma glucose and the HbAlc are not optimal screening modalities for the diagnosis of new diabetes in previously undiagnosed Asian indian community participants. Ethn Dis 2018;28:19-24.

10. Kumar PR, Bhansali A, Ravikiran M, Bhansali S, Dutta P, Thakur JS, et al. Utility of glycated hemoglobin in diagnosing type 2 diabetes mellitus: A community-based study. J Clin Endocrinol Metab 2010;95:2832-5

11. Mohan V, Vijayachandrika V, Gokulakrishnan K, Anjana RM, Ganesan A, Weber MB, et al. A1C cut points to define various glucose intolerance groups in Asian Indians. Diabetes Care 2010;33:515-9.

12. Herman WH, Ye W, Griffin SJ, Simmons RK, Davies MJ, Khunti $\mathrm{K}$, et al. Early detection and treatment of type 2 diabetes reduce cardiovascular morbidity and mortality: A simulation of the results of the anglo-danish-dutch study of intensive treatment in people with screen-detected diabetes in primary care (ADDITION-Europe). Diabetes Care 2015:38:1449-55.

13. Unnikrishnan R, Mohan V. Why screening for type 2 diabetes is necessary even in poor resource settings. J Diabetes Complications 2015;29:961-4.

14. Einarson TR, Bereza BG, Acs A, Jensen R. Systematic literature review of the health economic implications of early detection by screening populations at risk for type 2 diabetes. Curr Med Res Opin 2017;33:331-58

15. American Diabetes Association. 3. Prevention or delay of type 2 diabetes: Standards of medical care in diabetes-2019. Diabetes Care 2019;42:S29-33.

16. Anjana RM, Deepa M, Pradeepa R, Mahanta J, Narain K, Das HK, et al. Prevalence of diabetes and prediabetes in 15 states of India: Results from the ICMR-INDIAB population-based cross-sectional study. Lancet Diabetes Endocrinol 2017;5:585-96.

17. Madhu SV, Sandeep G, Mishra BK, Aslam M. High prevalence of diabetes, prediabetes and obesity among residents of East Delhi - The Delhi Urban diabetes survey (DUDS). Diabetes Metab Syndr 2018;12:923-7.

18. Majra JP, Verma R. Opportunistic screening for random blood glucose level among adults attending a rural tertiary care centre in Haryana during world health day observation activity. Int J Community Med Public Health 2017;4:1951-6.

19. Venugopal V, Selvaraj K, Majumdar A. Opportunistic screening for diabetes mellitus among adults attending a primary health center in Puducherry. Int J Med Sci Public Health 2015;4:1206-11.

20. Ramachandran A, Snehalatha C, Vijay V, Wareham NJ, Colagiuri S. Derivation and validation of diabetes risk score for urban Asian Indians. Diabetes Res Clin Pract 2005;70:63-70.

21. Mohan V, Deepa R, Deepa M, Somannavar S, Datta M. A simplified Indian diabetes Risk score for screening for undiagnosed diabetic subjects. J Assoc Physicians India 2005;53:759-63.

22. Mohan V, Vassy JL, Pradeepa R, Deepa M, Subashini S. The Indian type 2 diabetes risk score also helps identify those at risk of macrovasvular disease and neuropathy (CURES-77). J Assoc Physicians India 2010;58:430-3.

23. Ramachandran A, Snehalatha C, Ram J, Selvam S, Simon M, Nanditha A, et al. Effectiveness of mobile phone messaging in prevention of type 2 diabetes by lifestyle modification in men in India: A prospective, parallel-group, randomised controlled trial. Lancet Diabetes Endocrinol 2013;1:191-8.

24. Priya M, Anjana RM, Chiwanga FS, Gokulakrishnan K, Deepa M, Mohan V. 1-hour venous plasma glucose and incident prediabetes and diabetes in Asian indians. Diabetes Technol Ther 2013;15:497-502.

25. Inzucchi SE, Bergenstal RM, Buse JB, Diamant M, Ferrannini E, Nauck M, et al. Management of hyperglycemia in type 2 diabetes, 2015: A patient-centered approach: Update to a position statement of the American Diabetes Association and the European Association for the Study of Diabetes. Diabetes Care 2015;38:140-9.

26. Bowen ME, Xuan L, Lingvay I, Halm EA. Random blood glucose: A robust risk factor for type 2 diabetes. J Clin Endocrinol Metab 2015;100:1503-10.

27. Bowen ME, Xuan L, Lingvay I, Halm EA. Performance of a random glucose case-finding strategy to detect undiagnosed diabetes. Am J Prev Med 2017;52:710-6

28. Somannavar S, Ganesan A, Deepa M, Datta M, Mohan V. Random capillary blood glucose cut points for diabetes and pre-diabetes derived from community-based opportunistic screening in India. Diabetes Care 2009;32:641-3.

29. Elman K, Wainstein J, Boaz M, Jakubowicz D, Bar-Dayan Y. Random blood glucose screening at a public health station encouraged high risk subjects to make lifestyle changes. Int J Clin Pract 2017;71:e12984. doi: 10.1111/ijcp.12984.

30. Barry E, Roberts S, Oke J, Vijayaraghavan S, Normansell R, Greenhalgh T. Efficacy and effectiveness of screen and treat policies in prevention of type 2 diabetes: Systematic review and meta-analysis of screening tests and interventions. BMJ 2017;356:16538.

31. Gupta Y, Kapoor D, Desai A, Praveen D, Joshi R, Rozati R, et al. Conversion of gestational diabetes mellitus to future Type 2 diabetes mellitus and the predictive value of HbA1c in an Indian cohort. Diabet Med 2017;34:37-43.

32. Bertran EA, Berlie HD, Taylor A, Divine G, Jaber LA. Diagnostic performance of $\mathrm{HbA} 1 \mathrm{c}$ for diabetes in Arab vs. European populations: A systematic review and meta-analysis. Diabet Med 2017;34:156-66.

33. Cavagnolli G, Pimentel AL, Freitas PA, Gross JL, Camargo JL. Effect of ethnicity on HbA1c levels in individuals without diabetes: Systematic review and meta-analysis. PLoS One 2017;12:e0171315.

34. Hellgren M, Hjörleifsdottir Steiner K, Bennet L. Haemoglobin A1c as a screening tool for type 2 diabetes and prediabetes in populations of Swedish and Middle-East ancestry. Prim Care Diabetes 2017;11:337-43.

35. Madhu SV, Raj A, Gupta S, Giri S, Rusia U. Effect of iron deficiency anemia and iron supplementation on HbAlc levels - Implications for diagnosis of prediabetes and diabetes mellitus in Asian Indians. Clin Chim Acta 2017;468:225-9.

36. Unnikrishnan R, Anjana RM, Deepa M, Pradeepa R, Joshi SR, Bhansali A, et al. Glycemic control among individuals with self-reported diabetes in India - The ICMR-INDIAB Study. Diabetes Technol Ther 2014;16:596-603.

37. Bellamy L, Casas JP, Hingorani AD, Williams D. Type 2 diabetes mellitus after gestational diabetes: A systematic review and meta-analysis. Lancet 2009;373:1773-9. 
38. Retnakaran R. Glucose tolerance status in pregnancy: A window to the future risk of diabetes and cardiovascular disease in young women. Curr Diabetes Rev 2009;5:239-44.

39. Damm P. Future risk of diabetes in mother and child after gestational diabetes mellitus. Int J Gynaecol Obstet 2009;104 Suppl 1:S25-6.

40. Madhu S. Diabetes in pregnancy - A critical window of opportunity. Int J Diabetes Dev Ctries 2018;38:1-3.

41. Wabitsch M, Hauner H, Hertrampf M, Muche R, Hay B, Mayer H, et al. Type II diabetes mellitus and impaired glucose regulation in Caucasian children and adolescents with obesity living in Germany. Int J Obes Relat Metab Disord 2004;28:307-13.

42. Anjana RM, Pradeepa R, Deepa M, Datta M, Sudha V, Unnikrishnan R, et al. Prevalence of diabetes and prediabetes (impaired fasting glucose and/or impaired glucose tolerance) in urban and rural India: Phase I results of the Indian Council of Medical Research-INdia DIABetes (ICMR-INDIAB) study. Diabetologia 2011;54:3022-7.

43. Ramachandran A, Ma RC, Snehalatha C. Diabetes in Asia. Lancet 2010;375:408-18.

44. Ramachandran A, Snehalatha C, Viswanathan V, Viswanathan M, Haffner SM. Risk of noninsulin dependent diabetes mellitus conferred by obesity and central adiposity in different ethnic groups: A comparative analysis between Asian Indians, Mexican Americans and Whites. Diabetes Res Clin Pract 1997;36:121-5.

45. Sharp PS, Mohan V, Levy JC, Mather HM, Kohner EM. Insulin resistance in patients of Asian Indian and European origin with non-insulin dependent diabetes. Horm Metab Res 1987;19:84-5.

46. Anjana RM, Shanthi Rani CS, Deepa M, Pradeepa R, Sudha V, Divya Nair $\mathrm{H}$, et al. Incidence of diabetes and prediabetes and predictors of progression among Asian Indians: 10-year follow-up of the Chennai urban rural epidemiology study (CURES). Diabetes Care 2015;38:1441-8.

47. Dasappa H, Fathima FN, Prabhakar R, Sarin S. Prevalence of diabetes and pre-diabetes and assessments of their risk factors in urban slums of Bangalore. J Family Med Prim Care 2015;4:399-404.

48. Muthunarayanan L, Ramraj B, Russel JK. Prevalence of prediabetes and its associated risk factors among rural adults in Tamil Nadu. Arch Med Health Sci 2015;3:178.

49. Tripathy JP, Thakur JS, Jeet G, Chawla S, Jain S, Pal A, et al. Prevalence and risk factors of diabetes in a large community-based study in North India: Results from a STEPS survey in Punjab, India. Diabetol Metab Syndr 2017;9:8.

50. Pratyush DD, Tiwari S, Singh S, Singh SK. Risk factors of diabetes in North Indians with metabolic syndrome. Diabetes Metab Syndr 2016;10:S68-71.

51. Patel N, Deshpande S, Godbole V. Awareness and approach towards diagnosis and treatment of diabetes type 2 and its complication among general practioners of western Vadodara. Int J Diabetes Dev Ctries 2015;35:138-42.

52. Rawal LB, Tapp RJ, Williams ED, Chan C, Yasin S, Oldenburg B. Prevention of type 2 diabetes and its complications in developing countries: A review. Int J Behav Med 2012;19:121-33.

53. Acharya AS, Singh A, Dhiman B. Assessment of diabetes risk in an adult population using Indian diabetes risk Score in an Urban resettlement colony of Delhi. J Assoc Physicians India 2017;65:46-51.

54. Bhadoria AS, Kasar PK, Toppo NA. Validation of Indian diabetic risk score in diagnosing type 2 diabetes mellitus against high fasting blood sugar levels among adult population of central India. Biomed J 2015;38:359-60.

55. Gupta RK, Shora TN, Verma AK. Utility of MDRF-IDRS (Madras Diabetes Research Foundation-Indian Diabetes Risk Score) as a tool to assess risk for diabetes - A study from north-west India. Int J Diabetes Dev Ctries 2015;35:570-2

56. Nagalingam S, Sundaramoorthy K, Arumugam B. Screening for diabetes using Indian diabetes risk score. Int J Adv Med 2016;3:415-8.

57. Rajput M, Garg D, Rajput R. Validation of simplified Indian diabetes risk Score for screening undiagnosed diabetes in an urban setting of Haryana. Diabetes Metab Syndr 2017;11 Suppl 2:S539-42.

58. Sathish T, Oldenburg B, Tapp RJ, Shaw JE, Wolfe R, Sajitha B, et al. Baseline characteristics of participants in the Kerala diabetes prevention program: A cluster randomized controlled trial of lifestyle intervention in Asian Indians. Diabet Med 2017;34:647-53.

59. Priscilla S, Nanditha A, Simon M, Satheesh K, Kumar S, Shetty AS, et al. A pragmatic and scalable strategy using mobile technology to promote sustained lifestyle changes to prevent type 2 diabetes in India-Outcome of screening. Diabetes Res Clin Pract 2015;110:335-40.

60. Chaturvedi N. The burden of diabetes and its complications: Trends and implications for intervention. Diabetes Res Clin Pract 2007;76 Suppl 1:S3-12.

61. Madhu SV. World diabetes day 2015: Healthy living \& diabetes. Indian J Med Res 2015;142:503-6.

62. Edwardson CL, Gray LJ, Yates T. Detection and early lifestyle intervention in those at risk of type 2 diabetes. EMJ Diabet 2014;2:48-57.

63. Ramachandran A, Snehalatha C, Yamuna A, Mary S, Ping Z. Cost-effectiveness of the interventions in the primary prevention of diabetes among Asian Indians: Within-trial results of the Indian Diabetes Prevention Programme (IDPP). Diabetes Care 2007;30:2548-52.

64. Ramachandran A, Arun N, Shetty AS, Snehalatha C. Efficacy of primary prevention interventions when fasting and postglucose dysglycemia coexist: Analysis of the Indian Diabetes prevention programmes (IDPP-1 and IDPP-2). Diabetes Care 2010;33:2164-8.

65. Ramachandran A, Snehalatha C, Mary S, Mukesh B, Bhaskar AD, Vijay $\mathrm{V}$, et al. The Indian Diabetes prevention programme shows that lifestyle modification and metformin prevent type 2 diabetes in Asian Indian subjects with impaired glucose tolerance (IDPP-1). Diabetologia 2006;49:289-97.

66. Weber MB, Ranjani H, Staimez LR, Anjana RM, Ali MK, Narayan KM, et al. The stepwise approach to diabetes prevention: Results from the D-CLIP randomized controlled trial. Diabetes Care 2016;39:1760-7.

67. Kosaka K, Noda M, Kuzuya T. Prevention of type 2 diabetes by lifestyle intervention: A Japanese trial in IGT males. Diabetes Res Clin Pract 2005;67:152-62.

68. Pan XR, Li GW, Hu YH, Wang JX, Yang WY, An ZX, et al. Effects of diet and exercise in preventing NIDDM in people with impaired glucose tolerance. The Da Qing IGT and Diabetes Study. Diabetes Care 1997;20:537-44.

69. Saper RB, Phillips RS, Sehgal A, Khouri N, Davis RB, Paquin J, et al. Lead, mercury, and arsenic in US-and Indian-manufactured Ayurvedic medicines sold via the Internet. JAMA 2008;300:915-23.

70. Pittas AG, Dawson-Hughes B, Sheehan P, Ware JH, Knowler WC, Aroda VR, et al. Vitamin D supplementation and prevention of type 2 diabetes. N Engl J Med 2019;381:520-30.

71. Jadhav RA, Hazari A, Monterio A, Kumar S, Maiya AG. Effect of physical activity intervention in prediabetes: A systematic review with meta-analysis. J Phys Act Health 2017;14:745-55.

72. Cappuccio FP, D'Elia L, Strazzullo P, Miller MA. Quantity and quality of sleep and incidence of type 2 diabetes: A systematic review and meta-analysis. Diabetes Care 2010;33:414-20.

73. Cedernaes J, Schiöth HB, Benedict C. Determinants of shortened, disrupted, and mistimed sleep and associated metabolic health consequences in healthy humans. Diabetes 2015;64:1073-80.

74. Shan Z, Ma H, Xie M, Yan P, Guo Y, Bao W, et al. Sleep duration and risk of type 2 diabetes: A meta-analysis of prospective studies. Diabetes Care 2015;38:529-37.

75. Balagopal P, Kamalamma N, Patel TG, Misra R. A community-based diabetes prevention and management education program in a rural village in India. Diabetes Care 2008;31:1097-104.

76. Ramachandran A, Kumar R, Nanditha A. mDiabetes initiative using text messages to improve lifestyle and health-seeking behaviour in India. BMJ Innov 2018;4:155-62.

77. World Health Organisation. mHealth: New Horizons for Health Through Mobile Technologies. Geneva: Global Observatory for eHealth Series; 2011.

78. Modi S, Borges V, Chandalia H. Low carbohydrate or high carbohydrate: What is right? Review. Int J Diabetes Dev Ctries 2005;25:58-62.

79. Trivedi B, Maniyar KT, Patel B. Effect of fibre diet (guar) on cholesterol, blood glucose and body weight. Int J Diabetes Dev Ctries 1999;19:31-3.

80. Franz MJ, Zhang Z, Venn BJ. Lifestyle interventions to stem the tide of type 2 diabetes. In: Nutrition Guide for Physicians and Related Healthcare Professionals. Springer; 2017. p. 103-12. 
81. Sekar V, Sundaram A, Lakshmi B. The effect of modified pulsecarbohydrate diet on weight and $\mathrm{HbA} 1 \mathrm{C}$ in type 2 diabetic patients. Int J Diabetes Dev Ctries 2006;26:16-18.

82. Mohan D, Raj D, Shanthirani CS, Datta M, Unwin NC, Kapur A, et al. Awareness and knowledge of diabetes in Chennai-the Chennai Urban rural epidemiology study [CURES-9]. J Assoc Physicians India 2005;53:283-7.

83. Somannavar S, Lanthorn H, Deepa M, Pradeepa R, Rema M, Mohan V. Increased awareness about diabetes and its complications in a whole city: Effectiveness of the "prevention, awareness, counselling and evaluation" [PACE] Diabetes Project [PACE-6]. J Assoc Physicians India 2008;56:497-502.

84. Somannavar S, Lanthorn H, Pradeepa R, Narayanan V, Rema M, Mohan V. Prevention awareness counselling and evaluation (PACE) diabetes project: A mega multi-pronged program for diabetes awareness and prevention in South India (PACE- 5). J Assoc Physicians India 2008;56:429-35.

85. Gaddam A, Galla C, Thummisetti S, Marikanty RK, Palanisamy UD, Rao PV. Role of Fenugreek in the prevention of type 2 diabetes mellitus in prediabetes. J Diabetes Metab Disord 2015;14:74.

86. Forouhi NG, Misra A, Mohan V, Taylor R, Yancy W. Dietary and nutritional approaches for prevention and management of type 2 diabetes. BMJ 2018;361:k2234.

87. Dixit AA, Azar KM, Gardner CD, Palaniappan LP. Incorporation of whole, ancient grains into a modern Asian Indian diet to reduce the burden of chronic disease. Nutr Rev 2011;69:479-88.

88. Misra A, Singhal N, Sivakumar B, Bhagat N, Jaiswal A, Khurana L. Nutrition transition in India: Secular trends in dietary intake and their relationship to diet-related non-communicable diseases. J Diabetes 2011;3:278-92.

89. Popkin BM. Nutrition transition and the global diabetes epidemic. Curr Diab Rep 2015;15:64.

90. Davies MJ, D'Alessio DA, Fradkin J, Kernan WN, Mathieu C, Mingrone G, et al. Management of hyperglycaemia in type 2 diabetes, 2018. A consensus report by the American Diabetes Association (ADA) and the European Association for the Study of Diabetes (EASD). Diabetologia 2018;61:2461-98.

91. Garber AJ, Abrahamson MJ, Barzilay JI, Blonde L, Bloomgarden ZT, Bush MA, et al. Consensus statement by the American association of clinical endocrinologists and American college of endocrinology on the comprehensive type 2 diabetes management algorithm - 2018 executive summary. Endocr Pract 2018;24:91-120.

92. Baruah MP, Kalra S, Unnikrishnan AG, Raza SA, Somasundaram N, John M, et al. Management of hyperglycemia in geriatric patients with diabetes mellitus: South Asian consensus guidelines. Indian J Endocrinol Metab 2011:15:75-90.

93. Mathews E, Thomas E, Absetz P, D’Esposito F, Aziz Z, Balachandran S, et al. Cultural adaptation of a peer-led lifestyle intervention program for diabetes prevention in India: The Kerala diabetes prevention program (K-DPP). BMC Public Health 2018;17:974.

94. Gulati S, Misra A, Tiwari R, Sharma M, Pandey RM, Yadav CP. Effect of high-protein meal replacement on weight and cardiometabolic profile in overweight/obese Asian Indians in North India. Br J Nutr 2017;117:1531-40.

95. Malik VS, Sudha V, Wedick NM, RamyaBai M, Vijayalakshmi P, Lakshmipriya N, et al. Substituting brown rice for white rice on diabetes risk factors in India: A randomised controlled trial. Br J Nutr 2019;121:1389-97.

96. Pi-Sunyer X. The look AHEAD Trial: A review and discussion of its outcomes. Curr Nutr Rep 2014;3:387-91.

97. Myers EF, Trostler N, Varsha V, Voet H. Insights from the diabetes in India nutrition guidelines study: Adopting innovations using a knowledge transfer model. Top Clin Nutr 2017;32:69-86.

98. Gray A. Nutritional recommendations for individuals with diabetes. In: Feingold KR, Anawalt B, Boyce A, editors. Endotext. South Dartmouth (MA); 2000.

99. Jung $\mathrm{CH}$, Choi KM. Impact of high-carbohydrate diet on metabolic parameters in patients with type 2 diabetes. Nutrients 2017;9. pii: E322.

100. Mohan V, Ramachandran A, Viswanathan M. Dietary therapy of diabetes, evaluation of the high carbohydrate high fiber diet therapy. Recent Adv Diabetes 1983;80:657-63.

101. Viswanathan M, Mohan V, Ramakrishna A. Long-term experience with high-carbohydrate high-fiber diets in Indian diabetic patients. Diab Croat 1984;13:163-74.

102. Anderson JW, Ward K. Long-term effects of high-carbohydrate, high-fiber diets on glucose and lipid metabolism: A preliminary report on patients with diabetes. Diabetes Care 1978;1:77-82.

103. de Natale C, Annuzzi G, Bozzetto L, Mazzarella R, Costabile G, Ciano $\mathrm{O}$, et al. Effects of a plant-based high-carbohydrate/highfiber diet versus high-monounsaturated fat/low-carbohydrate diet on postprandial lipids in type 2 diabetic patients. Diabetes Care 2009;32:2168-73.

104. Sylvetsky AC, Edelstein SL, Walford G, Boyko EJ, Horton ES, Ibebuogu UN, et al. A high-carbohydrate, high-fiber, low-fat diet results in weight loss among adults at high risk of type 2 diabetes. $\mathrm{J}$ Nutr 2017:147:2060-6.

105. Chandalia M, Garg A, Lutjohann D, von Bergmann K, Grundy SM, Brinkley LJ. Beneficial effects of high dietary fiber intake in patients with type 2 diabetes mellitus. N Engl J Med 2000;342:1392-8.

106. Misra A, Khurana L, Isharwal S, Bhardwaj S. South Asian diets and insulin resistance. Br J Nutr 2009;101:465-73.

107. Mohan V, Radhika G, Sathya RM, Tamil SR, Ganesan A, Sudha V. Dietary carbohydrates, glycaemic load, food groups and newly detected type 2 diabetes among urban Asian Indian population in Chennai, India (Chennai Urban Rural Epidemiology Study 59). Br J Nutr 2009; 102:1498-506.

108. Radhika G, van Dam RM, Sudha V, Ganesan A, Mohan V. Refined grain consumption and the metabolic syndrome in urban Asian Indians (Chennai Urban Rural Epidemiology Study 57). Metabolism 2009;58:675-81.

109. Radhika G, Sathya RM, Ganesan A, Saroja R, Vijayalakshmi P, Sudha V, et al. Dietary profile of urban adult population in South India in the context of chronic disease epidemiology (CURES-68). Public Health Nutr 2011;14:591-8.

110. Mohan V, Ruchi V, Gayathri R, Bai MR, Sudha V, Anjana RM, et al. Slowing the diabetes epidemic in the World Health Organization South-East Asia Region: The role of diet and physical activity. WHO South East Asia J Public Health 2016;5:5-16.

111. Mohan V, Spiegelman D, Sudha V, Gayathri R, Hong B, Praseena K, et al. Effect of brown rice, white rice, and brown rice with legumes on blood glucose and insulin responses in overweight Asian Indians: A randomized controlled trial. Diabetes Technol Ther 2014;16:317-25.

112. Wedick NM, Sudha V, Spiegelman D, Bai MR, Malik VS, Venkatachalam SS, et al. Study design and methods for a randomized crossover trial substituting brown rice for white rice on diabetes risk factors in India. Int J Food Sci Nutr 2015;66:797-804.

113. Boers HM, MacAulay K, Murray P, Dobriyal R, Mela DJ, Spreeuwenberg MA. Efficacy of fibre additions to flatbread flour mixes for reducing post-meal glucose and insulin responses in healthy Indian subjects. Br J Nutr 2017;117:386-94.

114. Gulati S, Misra A, Pandey RM. Effects of $3 \mathrm{~g}$ of soluble fiber from oats on lipid levels of Asian Indians - A randomized controlled, parallel arm study. Lipids Health Dis 2017;16:71.

115. Radhika G, Sumathi C, Ganesan A, Sudha V, Jeya Kumar Henry C, Mohan V. Glycaemic index of Indian flatbreads (rotis) prepared using whole wheat flour and 'atta mix'-added whole wheat flour. Br J Nutr 2010;103:1642-7.

116. Thondre PS, Henry CJ. High-molecular-weight barley beta-glucan in chapatis (unleavened Indian flatbread) lowers glycemic index. Nutr Res 2009;29:480-6.

117. Gulati S, Misra A. Sugar intake, obesity, and diabetes in India. Nutrients 2014;6:5955-74.

118. Oh R, Uppaluri KR. Low Carbohydrate Diet. Treasure Island (FL): StatPearls; 2019.

119. Sackner-Bernstein J, Kanter D, Kaul S. Dietary intervention for overweight and obese adults: Comparison of low-carbohydrate and low-fat diets. A meta-analysis. PLoS One 2015;10:e0139817.

120. Srivastava S. Low carbohydrate diet in management of obesity - A short review. Int J Diabetes Dev Ctries 25:42-5. 
121. Saslow LR, Mason AE, Kim S, Goldman V, Ploutz-Snyder R, Bayandorian $\mathrm{H}$, et al. An online intervention comparing a very low-carbohydrate ketogenic diet and lifestyle recommendations versus a plate method diet in overweight individuals with type 2 diabetes: A randomized controlled trial. J Med Internet Res 2017;19:e36.

122. Saslow LR, Daubenmier JJ, Moskowitz JT, Kim S, Murphy EJ, Phinney SD, et al. Twelve-month outcomes of a randomized trial of a moderate-carbohydrate versus very low-carbohydrate diet in overweight adults with type 2 diabetes mellitus or prediabetes. Nutr Diabetes 2017;7:304.

123. Hussain TA, Mathew TC, Dashti AA, Asfar S, Al-Zaid N, Dashti HM. Effect of low-calorie versus low-carbohydrate ketogenic diet in type 2 diabetes. Nutrition 2012;28:1016-21.

124. Chandalia HB, Neogi R, Mehta S. Mechanism of low glycemic index of pulses and pulse- incorporated cereal foods. Int J Diabetes Dev Ctries 1992;12:9-11.

125. Shobana S, Kumari SR, Malleshi NG, Ali SZ. Glycemic response of rice, wheat and finger millet based diabetic food formulations in normoglycemic subjects. Int J Food Sci Nutr 2007;58:363-72.

126. Ghosh JM. Trial of low glycemic diet and acarbose therapy for control of post-prandial hyperglycemia in type 2 diabetes mellitus: Preliminary report. Int J Diabetes Dev Ctries 2005;25:80-4.

127. Sekar V, Sundaram A, Lakshmi B. The effect of modified pulsecarbohydrate diet on weight and $\mathrm{HbA1C}$ in type 2 diabetic patients. Int J Diabetes Dev Ctries 2006;26:16-8.

128. Viguiliouk E, Kendall CW, Blanco Mejia S, Cozma AI, Ha V, Mirrahimi A, et al. Effect of tree nuts on glycemic control in diabetes: A systematic review and meta-analysis of randomized controlled dietary trials. PLoS One 2014;9:e103376.

129. Green R, Milner J, Joy EJ, Agrawal S, Dangour AD. Dietary patterns in India: A systematic review. Br J Nutr 2016;116:142-8.

130. Becerra-Tomás N, Díaz-López A, Rosique-Esteban N, Ros E, Buil-Cosiales P, Corella D, et al. Legume consumption is inversely associated with type 2 diabetes incidence in adults: A prospective assessment from the PREDIMED study. Clin Nutr 2018;37:906-13.

131. Narasimhan S, Nagarajan L, Vaidya R, Gunasekaran G, Rajagopal G, Parthasarathy $\mathrm{V}$, et al. Dietary fat intake and its association with risk of selected components of the metabolic syndrome among rural South Indians. Indian J Endocrinol Metab 2016;20:47-54.

132. Lakshmipriya N, Gayathri R, Praseena K, Vijayalakshmi P, Geetha G, Sudha V, et al. Type of vegetable oils used in cooking and risk of metabolic syndrome among Asian Indians. Int J Food Sci Nutr 2013;64:131-9.

133. Nigam P, Bhatt S, Misra A, Chadha DS, Vaidya M, Dasgupta J, et al. Effect of a 6-month intervention with cooking oils containing a high concentration of monounsaturated fatty acids (olive and canola oils) compared with control oil in male Asian Indians with nonalcoholic fatty liver disease. Diabetes Technol Ther 2014;16:255-61.

134. Gulati S, Misra A. Abdominal obesity and type 2 diabetes in Asian Indians: Dietary strategies including edible oils, cooking practices and sugar intake. Eur J Clin Nutr 2017;71:850-7.

135. Gulati S, Misra A, Sharma M. Dietary fats and oils in India. Curr Diabetes Rev 2017;13:438-43.

136. Bhardwaj S, Passi SJ, Misra A, Pant KK, Anwar K, Pandey RM, et al. Effect of heating/reheating of fats/oils, as used by Asian Indians, on trans fatty acid formation. Food Chem 2016;212:663-70.

137. Kakde S, Bhopal RS, Bhardwaj S, Misra A. Urbanized South Asians' susceptibility to coronary heart disease: The high-heat food preparation hypothesis. Nutrition 2017;33:216-24.

138. Lattimer JM, Haub MD. Effects of dietary fiber and its components on metabolic health. Nutrients 2010;2:1266-89.

139. Mehta K, Kaur A. Dietary Fibre. Review. Int J Diabetes Dev Ctries 1992;12:12-8

140. Liu AG, Most MM, Brashear MM, Johnson WD, Cefalu WT, Greenway FL. Reducing the glycemic index or carbohydrate content of mixed meals reduces postprandial glycemia and insulinemia over the entire day but does not affect satiety. Diabetes Care 2012;35:1633-7.

141. Weickert MO, Pfeiffer AF. Impact of dietary fiber consumption on insulin resistance and the prevention of type 2 diabetes. J Nutr 2018;148:7-12.
142. Narayan S, Lakshmipriya N, Vaidya R, Bai MR, Sudha V, Krishnaswamy K, et al. Association of dietary fiber intake with serum total cholesterol and low density lipoprotein cholesterol levels in Urban Asian-Indian adults with type 2 diabetes. Indian J Endocrinol Metab 2014;18:624-30.

143. Yao B, Fang H, Xu W, Yan Y, Xu H, Liu Y, et al. Dietary fiber intake and risk of type 2 diabetes: A dose-response analysis of prospective studies. Eur J Epidemiol 2014;29:79-88.

144. Emadian A, Thompson J. A mixed-methods examination of physical activity and sedentary time in overweight and obese South Asian men living in the United Kingdom. Int J Environ Res Public Health 2017;14. pii: E348

145. Lee IM, Shiroma EJ, Lobelo F, Puska P, Blair SN, Katzmarzyk PT, et al. Effect of physical inactivity on major non-communicable diseases worldwide: An analysis of burden of disease and life expectancy. Lancet 2012;380:219-29.

146. Boulé NG, Kenny GP, Haddad E, Wells GA, Sigal RJ. Meta-analysis of the effect of structured exercise training on cardiorespiratory fitness in Type 2 diabetes mellitus. Diabetologia 2003;46:1071-81.

147. Iliodromiti S, Ghouri N, Celis-Morales CA, Sattar N, Lumsden MA, Gill JM. Should physical activity recommendations for South Asian adults be ethnicity-specific? Evidence from a cross-sectional study of South Asian and white European men and women. PLoS One 2016;11:e0160024.

148. Hameed UA, Manzar D, Raza S, Shareef MY, Hussain ME. Resistance training leads to clinically meaningful improvements in control of glycemia and muscular strength in untrained middle-aged patients with type 2 diabetes mellitus. N Am J Med Sci 2012;4:336-43.

149. Hou Y, Lin L, Li W. Effect of combined training versus aerobic training alone on glucose control and risk factors for complications in type 2 diabetic patients: A meta-analysis. Int J Diabetes Dev Ctries 2015;35:524-32.

150. Aylin K, Arzu D, Sabri S, Handan TE, Ridvan A. The effect of combined resistance and home-based walking exercise in type 2 diabetes patients. Int J Diabetes Dev Ctries 2009;29:159-65.

151. Professional Practice Committee: Standards of medical care in diabetes-2018. Diabetes Care 2018;41:S3

152. Misra A, Nigam P, Hills AP, Chadha DS, Sharma V, Deepak KK, et al. Consensus physical activity guidelines for Asian Indians. Diabetes Technol Ther 2012;14:83-98.

153. Spahn JM, Reeves RS, Keim KS, Laquatra I, Kellogg M, Jortberg B, et al. State of the evidence regarding behavior change theories and strategies in nutrition counseling to facilitate health and food behavior change. J Am Diet Assoc 2010;110:879-91.

154. Vermunt PW, Milder IE, Wielaard F, Baan CA, Schelfhout JD, Westert GP, et al. Behavior change in a lifestyle intervention for type 2 diabetes prevention in Dutch primary care: Opportunities for intervention content. BMC Fam Pract 2013;14:78.

155. S. A, A. S, S. A, et al. Behavioural lifestyle intervention study (BLIS) in patients with type 2 diabetes in the United Arab Emirates: A randomized controlled trial. BMC Nutr 2015;1:37-45

156. Wang J, Cai C, Padhye N, Orlander P, Zare M. A behavioral lifestyle intervention enhanced with multiple-behavior self-monitoring using mobile and connected tools for underserved individuals with type 2 diabetes and comorbid overweight or obesity: Pilot comparative effectiveness trial. JMIR Mhealth Uhealth 2018;6:e92.

157. Baker MK, Simpson K, Lloyd B, Bauman AE, Singh MA. Behavioral strategies in diabetes prevention programs: A systematic review of randomized controlled trials. Diabetes Res Clin Pract 2011;91:1-2.

158. Wu Y, Ding Y, Tanaka Y, Zhang W. Risk factors contributing to type 2 diabetes and recent advances in the treatment and prevention. Int J Med Sci 2014;11:1185-200

159. Defronzo RA. Banting lecture. From the triumvirate to the ominous octet: A new paradigm for the treatment of type 2 diabetes mellitus. Diabetes 2009;58:773-95.

160. Wells JC, Pomeroy E, Walimbe SR, Popkin BM, Yajnik CS. The elevated susceptibility to diabetes in India: An evolutionary perspective. Front Public Health 2016;4:145.

161. Mehta SR, Kashyap AS, Das S. Diabetes mellitus in India: The modern scourge. Med J Armed Forces India 2009;65:50-4. 
162. Mohan V. Why are Indians more prone to diabetes? J Assoc Physicians India 2004;52:468-74.

163. Garber AJ, Abrahamson MJ, Barzilay JI, Blonde L, Bloomgarden ZT, Bush MA, et al. Consensus statement by the American association of clinical endocrinologists and American college of endocrinology on the comprehensive type 2 diabetes management algorithm - 2017 executive summary. Endocr Pract 2017;23:207-38.

164. Thrasher J. Pharmacologic management of type 2 diabetes mellitus: Available Therapies. Am J Med 2017;130:S4-17.

165. Holman R. Metformin as first choice in oral diabetes treatment: The UKPDS experience. Journ Annu Diabetol Hotel Dieu 2007:13-20.

166. Ripsin CM, Kang H, Urban RJ. Management of blood glucose in type 2 diabetes mellitus. Am Fam Physician 2009;79:29-36.

167. Rena G, Hardie DG, Pearson ER. The mechanisms of action of metformin. Diabetologia 2017;60:1577-85.

168. Effect of intensive blood-glucose control with metformin on complications in overweight patients with type 2 diabetes (UKPDS 34). UK Prospective Diabetes Study (UKPDS) Group. Lancet 1998;352:854-65.

169. Holman RR, Paul SK, Bethel MA, Matthews DR, Neil HA. 10-year follow-up of intensive glucose control in type 2 diabetes. N Engl J Med 2008;359:1577-89.

170. Rojas LB, Gomes MB. Metformin: An old but still the best treatment for type 2 diabetes. Diabetol Metab Syndr 2013;5:6.

171. Kalra S, Aamir AH, Raza A, Das AK, Azad Khan AK, Shrestha D, et al. Place of sulfonylureas in the management of type 2 diabetes mellitus in South Asia: A consensus statement. Indian J Endocrinol Metab 2015;19:577-96.

172. Sola D, Rossi L, Schianca GP, Maffioli P, Bigliocca M, Mella R, et al. Sulfonylureas and their use in clinical practice. Arch Med Sci 2015;11:840-8.

173. Weintraub NL. Impaired hypoxic coronary vasodilation and ATP-sensitive potassium channel function: A manifestation of diabetic microangiopathy in humans? Circ Res 2003;92:127-9.

174. Garratt KN, Brady PA, Hassinger NL, Grill DE, Terzic A, Holmes DR Jr. Sulfonylurea drugs increase early mortality in patients with diabetes mellitus after direct angioplasty for acute myocardial infarction. J Am Coll Cardiol 1999;33:119-24.

175. Varvaki Rados D, Catani Pinto L, Reck Remonti L, Bauermann Leitão C, Gross JL. The association between sulfonylurea use and all-cause and cardiovascular mortality: A meta-analysis with trial sequential analysis of randomized clinical trials. PLoS Med 2016;13:e1001992.

176. Kalra S, Bahendeka S, Sahay R, Ghosh S, MdF, Orabi A, et al. Consensus recommendations on sulfonylurea and sulfonylurea combinations in the management of type 2 diabetes mellitus - International task force. Indian J Endocrinol Metab 2018;22:132-57.

177. Davies MJ, D’Alessio DA, Fradkin J, Kernan WN, Mathieu C, Mingrone G, et al. Management of hyperglycemia in type 2 diabetes, 2018. A consensus report by the American Diabetes Association (ADA) and the European Association for the Study of Diabetes (EASD). Diabetes Care 2018;41:2669-701.

178. Guardado-Mendoza R, Prioletta A, Jiménez-Ceja LM, Sosale A, Folli F. The role of nateglinide and repaglinide, derivatives of meglitinide, in the treatment of type 2 diabetes mellitus. Arch Med Sci 2013;9:936-43.

179. Camp HS. Thiazolidinediones in diabetes: Current status and future outlook. Curr Opin Investig Drugs 2003;4:406-11.

180. Kahn SE, Haffner SM, Heise MA, Herman WH, Holman RR, Jones NP, et al. Glycemic durability of rosiglitazone, metformin, or glyburide monotherapy. N Engl J Med 2006;355:2427-43.

181. Nissen SE, Wolski K. Effect of rosiglitazone on the risk of myocardial infarction and death from cardiovascular causes. N Engl J Med 2007;356:2457-71.

182. Home PD, Pocock SJ, Beck-Nielsen H, Curtis PS, Gomis R, Hanefeld M, et al. Rosiglitazone evaluated for cardiovascular outcomes in oral agent combination therapy for type 2 diabetes (RECORD): A multicentre, randomised, open-label trial. Lancet 2009;373:2125-35.

183. Defronzo RA, Mehta RJ, Schnure JJ. Pleiotropic effects of thiazolidinediones: Implications for the treatment of patients with type 2 diabetes mellitus. Hosp Pract (1995) 2013;41:132-47.

184. Dormandy JA, Charbonnel B, Eckland DJ, Erdmann E,
Massi-Benedetti M, Moules IK, et al. Secondary prevention of macrovascular events in patients with type 2 diabetes in the PROactive Study (PROspective pioglitAzone Clinical Trial In macroVascular Events): A randomised controlled trial. Lancet 2005;366:1279-89.

185. Fonseca V, Rosenstock J, Patwardhan R, Salzman A. Effect of metformin and rosiglitazone combination therapy in patients with type 2 diabetes mellitus: A randomized controlled trial. JAMA 2000;283:1695-702.

186. Kernan WN, Viscoli CM, Furie KL, Young LH, Inzucchi SE, Gorman M, et al. Pioglitazone after Ischemic Stroke or Transient Ischemic Attack. N Engl J Med 2016;374:1321-31.

187. Spence JD, Viscoli CM, Inzucchi SE, Dearborn-Tomazos J, Ford GA, Gorman M, et al. Pioglitazone therapy in patients with stroke and prediabetes: A post hoc analysis of the IRIS Randomized Clinical Trial. JAMA Neurol 2019;76:526-35.

188. Lewis JD, Ferrara A, Peng T, Hedderson M, Bilker WB, Quesenberry CP Jr., et al. Risk of bladder cancer among diabetic patients treated with pioglitazone: Interim report of a longitudinal cohort study. Diabetes Care 2011;34:916-22.

189. Tang H, Shi W, Fu S, Wang T, Zhai S, Song Y, et al. Pioglitazone and bladder cancer risk: A systematic review and meta-analysis. Cancer Med 2018;7:1070-80.

190. Pai SA, Kshirsagar NA. Pioglitazone utilization, efficacy \& safety in Indian type 2 diabetic patients: A systematic review \& comparison with European Medicines Agency Assessment Report. Indian J Med Res 2016;144:672-81.

191. Cahn A, Raz I. Emerging gliptins for type 2 diabetes. Expert Opin Emerg Drugs 2013;18:245-58.

192. Pathak R, Bridgeman MB. Dipeptidyl peptidase-4 (DPP-4) Inhibitors in the management of diabetes. P T 2010;35:509-13.

193. Raz I, Hanefeld M, Xu L, Caria C, Williams-Herman D, Khatami H, et al. Efficacy and safety of the dipeptidyl peptidase-4 inhibitor sitagliptin as monotherapy in patients with type 2 diabetes mellitus. Diabetologia 2006; $49: 2564-71$.

194. Ahrén B. Use of DPP-4 inhibitors in type 2 diabetes: Focus on sitagliptin. Diabetes Metab Syndr Obes 2010;3:31-41.

195. Stein SA, Lamos EM, Davis SN. A review of the efficacy and safety of oral antidiabetic drugs. Expert Opin Drug Saf 2013;12:153-75.

196. Wu S, Hopper I, Skiba M, Krum H. Dipeptidyl peptidase-4 inhibitors and cardiovascular outcomes: Meta-analysis of randomized clinical trials with 55,141 participants. Cardiovasc Ther 2014;32:147-58.

197. Scirica BM, Bhatt DL, Braunwald E, Steg PG, Davidson J, Hirshberg B, et al. Saxagliptin and cardiovascular outcomes in patients with type 2 diabetes mellitus. N Engl J Med 2013;369:1317-26.

198. Zannad F, Cannon CP, Cushman WC, Bakris GL, Menon V, Perez AT, et al. Heart failure and mortality outcomes in patients with type 2 diabetes taking alogliptin versus placebo in EXAMINE: A multicentre, randomised, double-blind trial. Lancet 2015;385:2067-76.

199. FDA Drug Safety Communication: FDA adds Warnings about Heart Failure Risk to Labels of Type 2 Diabetes Medicines Containing Saxagliptin and Alogliptin; 2016. Available from: https://www.fda. gov/media/96895/download.

200. Rosenstock J, Perkovic V, Johansen OE, Cooper ME, Kahn SE, Marx N, et al. Effect of linagliptin vs. placebo on major cardiovascular events in adults with type 2 diabetes and high cardiovascular and renal risk: The CARMELINA randomized clinical trial. JAMA 2019;321:69-79.

201. Boehringer Ingelheim and Lilly's CAROLINA ${ }^{\circledR}$ Cardiovascular Outcome Trial for Trajenta ${ }^{\circ}$ Meets Primary Endpoint of non-Inferiority Compared to Glimepiride. Ingelheim, Germany and Indianapolis; 2019. Available from: https://www.boehringer-ingelheim.com/pressrelease/CAROLINA-top-line. [Last accessed on 2019 Aug 08].

202. DeFronzo RA, Davidson JA, Del Prato S. The role of the kidneys in glucose homeostasis: A new path towards normalizing glycaemia. Diabetes Obes Metab 2012;14:5-14.

203. Singh AK, Unnikrishnan AG, Zargar AH, Kumar A, Das AK, Saboo B, et al. Evidence-based consensus on positioning of SGLT2i in type 2 diabetes mellitus in Indians. Diabetes Ther 2019;10:393-428.

204. Markham A. Remogliflozin etabonate: First global approval. Drugs 2019;79:1157-61.

205. Zinman B, Wanner C, Lachin JM, Fitchett D, Bluhmki E, Hantel S, 
et al. Empagliffozin, cardiovascular outcomes, and mortality in type 2 diabetes. N Engl J Med 2015;373:2117-28.

206. Heerspink HJ, Desai M, Jardine M, Balis D, Meininger G, Perkovic V. Canagliflozin slows progression of renal function decline independently of glycemic effects. J Am Soc Nephrol 2017;28:368-75.

207. Neal B, Perkovic V, Mahaffey KW, de Zeeuw D, Fulcher G, Erondu N, et al. Canagliflozin and cardiovascular and renal events in type 2 diabetes. N Engl J Med 2017;377:644-57.

208. Rosenstock J, Aggarwal N, Polidori D, Zhao Y, Arbit D, Usiskin K, et al. Dose-ranging effects of canagliflozin, a sodium-glucose cotransporter 2 inhibitor, as add-on to metformin in subjects with type 2 diabetes. Diabetes Care 2012;35:1232-8

209. Baker WL, Buckley LF, Kelly MS, Bucheit JD, Parod ED, Brown R, et al. Effects of sodium-glucose cotransporter 2 inhibitors on 24-hour ambulatory blood pressure: A Systematic Review and Meta-Analysis. J Am Heart Assoc 2017;6. pii: E005686.

210. Nyirjesy P, Zhao Y, Ways K, Usiskin K. Evaluation of vulvovaginal symptoms and candida colonization in women with type 2 diabetes mellitus treated with canagliflozin, a sodium glucose co-transporter 2 inhibitor. Curr Med Res Opin 2012;28:1173-8.

211. Bailey CJ, Gross JL, Pieters A, Bastien A, List JF. Effect of dapagliflozin in patients with type 2 diabetes who have inadequate glycaemic control with metformin: A randomised, double-blind, placebo-controlled trial. Lancet 2010;375:2223-33

212. Grempler R, Thomas L, Eckhardt M, Himmelsbach F, Sauer A, Sharp DE, et al. Empagliflozin, a novel selective sodium glucose cotransporter-2 (SGLT-2) inhibitor: Characterisation and comparison with other SGLT-2 inhibitors. Diabetes Obes Metab 2012;14:83-90.

213. Stenlöf K, Cefalu WT, Kim KA, Alba M, Usiskin K, Tong C, et al. Efficacy and safety of canagliflozin monotherapy in subjects with type 2 diabetes mellitus inadequately controlled with diet and exercise. Diabetes Obes Metab 2013;15:372-82.

214. Yoon KH, Nishimura R, Lee J, Crowe S, Salsali A, Hach T, et al. Efficacy and safety of empagliflozin in patients with type 2 diabetes from Asian countries: Pooled data from four phase III trials. Diabetes Obes Metab 2016;18:1045-9.

215. Bischoff H. Pharmacology of alpha-glucosidase inhibition. Eur J Clin Invest 1994;24 Suppl 3:3-10.

216. Hoffmann J, Spengler M. Efficacy of 24-week monotherapy with acarbose, metformin, or placebo in dietary-treated NIDDM patients: The Essen-II Study. Am J Med 1997;103:483-90.

217. Halimi S, Le Berre MA, Grangé V. Efficacy and safety of acarbose add-on therapy in the treatment of overweight patients with Type 2 diabetes inadequately controlled with metformin: A double-blind, placebo-controlled study. Diabetes Res Clin Pract 2000;50:49-56.

218.Cavaiola TS PJ. Selecting amongst available pharmacological agents. In: Feingold KR, Anawalt B, Boyce A, editors. Endotext. Available from: https://www.ncbi.nlm.nih.gov/books/NBK425702/. [Last updated 2017 Mar 31].

219. American Diabetes Association. 9. Pharmacologic approaches to glycemic treatment: Standards of medical care in diabetes-2019. Diabetes Care 2019;42:S90-102.

220. Baruah MP, Kalra S, Bose S, Deka J. An Audit of Insulin Usage and Insulin Injection Practices in a Large Indian Cohort. Indian J Endocrinol Metab 2017;21:443-52.

221. Handelsman Y, Bloomgarden ZT, Grunberger G, Umpierrez G, Zimmerman RS, Bailey TS, et al. American association of clinical endocrinologists and American college of endocrinology - Clinical practice guidelines for developing a diabetes mellitus comprehensive care plan-2015. Endocr Pract 2015;21 Suppl 1:1-87.

222. Intensive blood-glucose control with sulphonylureas or insulin compared with conventional treatment and risk of complications in patients with type 2 diabetes (UKPDS 33). UK Prospective Diabetes Study (UKPDS) Group. Lancet 1998;352:837-53.

223. Diabetes Control and Complications Trial/Epidemiology of Diabetes Interventions and Complications Research Group, Lachin JM, Genuth S, Cleary P, Davis MD, Nathan DM. Retinopathy and nephropathy in patients with type 1 diabetes four years after a trial of intensive therapy. N Engl J Med 2000;342:381-9.

224. Raccah D. Basal insulin treatment intensification in patients with type
2 diabetes mellitus: A comprehensive systematic review of current options. Diabetes Metab 2017;43:110-24.

225. Kalra S, Baruah MP, Sahay RK, Unnikrishnan AG, Uppal S, Adetunji O. Glucagon-like peptide-1 receptor agonists in the treatment of type 2 diabetes: Past, present, and future. Indian J Endocrinol Metab 2016;20:254-67.

226. Inzucchi SE, Bergenstal RM, Buse JB, Diamant M, Ferrannini E, Nauck M, et al. Management of hyperglycaemia in type 2 diabetes: A patient-centered approach. Position statement of the American Diabetes Association (ADA) and the European Association for the Study of Diabetes (EASD). Diabetologia 2012;55:1577-96.

227. Ismail-Beigi $F$, Moghissi $E$, Tiktin $M$, Hirsch IB, Inzucchi SE, Genuth S. Individualizing glycemic targets in type 2 diabetes mellitus: Implications of recent clinical trials. Ann Intern Med 2011;154:554-9.

228. Edridge CL, Dunkley AJ, Bodicoat DH, Rose TC, Gray LJ, Davies MJ, et al. Prevalence and incidence of hypoglycaemia in 532,542 people with type 2 diabetes on oral therapies and insulin: A systematic review and meta-analysis of population based studies. PLoS One 2015;10:e126427.

229. Chandalia HB, Lamba PS, Chandalia SH, Singh DK, Modi SV, Shaikh SA. Weight gain in type 2 diabetics with different treatment modalities. Metab Syndr Relat Disord 2005;3:130-6.

230. Vos RC, van Avendonk MJ, Jansen H, Goudswaard AN, van den Donk M, Gorter K, et al. Insulin monotherapy compared with the addition of oral glucose-lowering agents to insulin for people with type 2 diabetes already on insulin therapy and inadequate glycaemic control. Cochrane Database Syst Rev 2016;9:CD006992.

231. Kalra S, Ghosal S, Shah P. Consensus on bridges for barriers to insulin therapy. J Assoc Physicians India 2017;65:23-30.

232. International Diabetes Federation Guideline Development Group. Global guideline for type 2 diabetes. Diabetes Res Clin Pract 2014; $104: 1-52$

233. Holman RR, Farmer AJ, Davies MJ, Levy JC, Darbyshire JL, Keenan JF, et al. Three-year efficacy of complex insulin regimens in type 2 diabetes. N Engl J Med 2009;361:1736-47.

234. Kalra S, Gupta Y. Insulin initiation: Bringing objectivity to choice. J Diabetes Metab Disord 2015;14:17.

235. Kim W, Egan JM. The role of incretins in glucose homeostasis and diabetes treatment. Pharmacol Rev 2008;60:470-512.

236. Bentley-Lewis R, Aguilar D, Riddle MC, Claggett B, Diaz R, Dickstein $\mathrm{K}$, et al. Rationale, design, and baseline characteristics in Evaluation of LIXisenatide in Acute Coronary Syndrome, a long-term cardiovascular end point trial of lixisenatide versus placebo. Am Heart J 2015; 169:631-8.e7.

237. Marso SP, Daniels GH, Brown-Frandsen K, Kristensen P, Mann JF, Nauck MA, et al. Liraglutide and cardiovascular outcomes in type 2 diabetes. N Engl J Med 2016;375:311-22.

238. The International Federation (IDF) Diabetes Atlas, $8^{\text {th }}$ ed. International Diabetes Federation; 2018. Available from: http://www.diabetesatlas. org/. [Last accessed on 2019 June 07].

239. Bennett WL, Maruthur NM, Singh S, Segal JB, Wilson LM, Chatterjee $\mathrm{R}$, et al. Comparative effectiveness and safety of medications for type 2 diabetes: An update including new drugs and 2-drug combinations. Ann Intern Med 2011;154:602-13.

240. Kesavadev J, Rajput R, Mathew J. Consensus statement on choice of insulin therapy in type 2 diabetes. Suppl J Assoc Phys India 2016:13-8.

241. Barnett AH, Huisman H, Jones R, von Eynatten M, Patel S, Woerle HJ. Linagliptin for patients aged 70 years or older with type 2 diabetes inadequately controlled with common antidiabetes treatments: A randomised, double-blind, placebo-controlled trial. Lancet 2013;382:1413-23

242. Chien MN, Lee CC, Chen WC. Effect of sitagliptin as add-on therapy in elderly type 2 diabetes patients with inadequate glycemic control in Taiwan. Int J Gerontol 2011;5:103-6.

243. Strain WD, Agarwal AS, Paldánius PM. Individualizing treatment targets for elderly patients with type 2 diabetes: Factors influencing clinical decision making in the 24-week, randomized INTERVAL study. Aging (Albany NY) 2017;9:769-77.

244. Strain WD, Lukashevich V, Kothny W, Hoellinger MJ, Paldánius PM. Individualised treatment targets for elderly patients with type 2 diabetes 
using vildagliptin add-on or lone therapy (INTERVAL): A 24 week, randomised, double-blind, placebo-controlled study. Lancet 2013;382:409-16.

245. Dardano A, Penno G, Del Prato S, Miccoli R. Optimal therapy of type 2 diabetes: A controversial challenge. Aging (Albany NY) 2014;6:187-206.

246. Josse RG, Chiasson JL, Ryan EA, Lau DC, Ross SA, Yale JF, et al. Acarbose in the treatment of elderly patients with type 2 diabetes. Diabetes Res Clin Pract 2003;59:37-42.

247. Dunning T, Sinclair A, Colagiuri S. New IDF Guideline for managing type 2 diabetes in older people. Diabetes Res Clin Pract 2014;103:538-40.

248. Karnieli E, Baeres FM, Dzida G, Ji Q, Ligthelm R, Ross S, et al. Observational study of once-daily insulin detemir in people with type 2 diabetes aged 75 years or older: A sub-analysis of data from the Study of Once daily LeVEmir (SOLVE). Drugs Aging 2013;30:167-75.

249. Pandya N, DiGenio A, Gao L, Patel M. Efficacy and safety of insulin glargine compared to other interventions in younger and older adults: A pooled analysis of nine open-label, randomized controlled trials in patients with type 2 diabetes. Drugs Aging 2013;30:429-38.

250. Lee P, Chang A, Blaum C, Vlajnic A, Gao L, Halter J. Comparison of safety and efficacy of insulin glargine and neutral protamine hagedorn insulin in older adults with type 2 diabetes mellitus: Results from a pooled analysis. J Am Geriatr Soc 2012;60:51-9.

251. American Diabetes Association. 8. Obesity management for the treatment of Type 2 diabetes: Standards of medical care in diabetes-2019. Diabetes Care 2019;42:s81-9.

252. Svacina S. Treatment of obese diabetics. Adv Exp Med Biol 2012;771:459-64.

253. Mearns ES, Sobieraj DM, White CM, Saulsberry WJ, Kohn CG, Doleh Y, et al. Comparative efficacy and safety of antidiabetic drug regimens added to metformin monotherapy in patients with type 2 diabetes: A network meta-analysis. PLoS One 2015;10:e125879.

254. Potts JE, Gray LJ, Brady EM, Khunti K, Davies MJ, Bodicoat DH. The effect of glucagon-like peptide 1 receptor agonists on weight loss in type 2 diabetes: A systematic review and mixed treatment comparison meta-analysis. PLoS One 2015;10:e126769.

255. Monami M, Nardini C, Mannucci E. Efficacy and safety of sodium glucose co-transport-2 inhibitors in type 2 diabetes: A meta-analysis of randomized clinical trials. Diabetes Obes Metab 2014;16:457-66.

256. Tosaki T, Kamiya H, Himeno T, Kato Y, Kondo M, Toyota K, et al. Sodium-glucose Co-transporter 2 inhibitors reduce the abdominal visceral fat area and may influence the renal function in patients with type 2 diabetes. Intern Med 2017;56:597-604.

257. Chen JF, Chang CM, Kuo MC, Tung SC, Tsao CF, Tsai CJ. Impact of baseline body mass index status on glucose lowering and weight change during sitagliptin treatment for type 2 diabetics. Diabetes Res Clin Pract 2016;120:8-14.

258. Kodera R, Shikata K, Nakamura A, Okazaki S, Nagase R, Nakatou T, et al. The glucose-lowering efficacy of sitagliptin in obese Japanese patients with type 2 diabetes. Intern Med 2017;56:605-13.

259. Agarwal P, Jindal C, Sapakal V. Efficacy and safety of teneligliptin in Indian patients with inadequately controlled type 2 diabetes mellitus: A randomized, double-blind study. Indian $\mathrm{J}$ Endocrinol Metab 2018;22:41-6.

260. Hassanein M, Abdallah K, Schweizer A. A double-blind, randomized trial, including frequent patient-physician contacts and Ramadan-focused advice, assessing vildagliptin and gliclazide in patients with type 2 diabetes fasting during Ramadan: The STEADFAST study. Vasc Health Risk Manag 2014;10:319-26.

261. Martin S, Kolb H, Beuth J, van Leendert R, Schneider B, Scherbaum WA. Change in patients' body weight after 12 months of treatment with glimepiride or glibenclamide in Type 2 diabetes: A multicentre retrospective cohort study. Diabetologia 2003;46:1611-7.

262. Zoungas S, Chalmers J, Neal B, Billot L, Li Q, Hirakawa Y, et al. Follow-up of blood-pressure lowering and glucose control in type 2 diabetes. N Engl J Med 2014;371:1392-406.

263. George AM, Jacob AG, Fogelfeld L. Lean diabetes mellitus: An emerging entity in the era of obesity. World J Diabetes 2015;6:613-20.

264. Abubaker M, Mishra P, Swami OC. Teneligliptin in Management of diabetic kidney disease: A review of place in therapy. J Clin Diagn Res 2017;11:OE05-9.

265. Kishimoto M. Teneligliptin: A DPP-4 inhibitor for the treatment of type 2 diabetes. Diabetes Metab Syndr Obes 2013;6:187-95.

266. Penno G, Garofolo M, Del Prato S. Dipeptidyl peptidase-4 inhibition in chronic kidney disease and potential for protection against diabetes-related renal injury. Nutr Metab Cardiovasc Dis 2016;26:361-73.

267. Russo E, Penno G, Del Prato S. Managing diabetic patients with moderate or severe renal impairment using DPP-4 inhibitors: Focus on vildagliptin. Diabetes Metab Syndr Obes 2013;6:161-70.

268. Ioannidis I. Diabetes treatment in patients with renal disease: Is the landscape clear enough? World J Diabetes 2014;5:651-8.

269. Madhu S. Use of oral anti-diabetic agents in diabetes with chronic kidney disease. Medicine 2011:157.

270. Urata H, Mori K, Emoto M, Yamazaki Y, Motoyama K, Morioka T, et al. Advantage of insulin glulisine over regular insulin in patients with type 2 diabetes and severe renal insufficiency. J Ren Nutr 2015;25:129-34.

271. National Kidney Foundation. KDOQI clinical practice guideline for diabetes and CKD: 2012 update. Am J Kidney Dis 2012;60:850-86.

272. Abe M, Kalantar-Zadeh K. Haemodialysis-induced hypoglycaemia and glycaemic disarrays. Nat Rev Nephrol 2015;11:302-13.

273. Moghissi ES. Treating patients with diabetes of long duration: GLP-1 receptor agonists and insulin in combination. J Am Osteopath Assoc 2014;114:S22-9.

274. Kalra S. Sodium glucose co-transporter-2 (SGLT2) inhibitors: A Review of their basic and clinical pharmacology. Diabetes Ther 2014;5:355-66.

275. Ray KK, Seshasai SR, Wijesuriya S, Sivakumaran R, Nethercott S, Preiss D, et al. Effect of intensive control of glucose on cardiovascular outcomes and death in patients with diabetes mellitus: A meta-analysis of randomised controlled trials. Lancet 2009;373:1765-72.

276. Gerstein HC, Colhoun HM, Dagenais GR, Diaz R, Lakshmanan M, Pais $\mathrm{P}$, et al. Design and baseline characteristics of participants in the Researching cardiovascular Events with a Weekly INcretin in Diabetes (REWIND) trial on the cardiovascular effects of dulaglutide. Diabetes Obes Metab 2018;20:42-9.

277. Hernandez AF, Green JB, Janmohamed S, D'Agostino RB Sr., Granger CB, Jones NP, et al. Albiglutide and cardiovascular outcomes in patients with type 2 diabetes and cardiovascular disease (Harmony Outcomes): A double-blind, randomised placebo-controlled trial. Lancet 2018;392:1519-29.

278. Mahaffey KW, Jardine MJ, Bompoint S. Canagliflozin and cardiovascular and renal outcomes in type 2 diabetes and chronic kidney disease in primary and secondary cardiovascular prevention groups: Results from the randomized CREDENCE trial. Circulation 2019.

279. Marso SP, Bain SC, Consoli A, Eliaschewitz FG, Jódar E, Leiter LA, et al. Semaglutide and cardiovascular outcomes in patients with type 2 diabetes. N Engl J Med 2016;375:1834-44.

280. Xu J, Rajaratnam R. Cardiovascular safety of non-insulin pharmacotherapy for type 2 diabetes. Cardiovasc Diabetol 2017;16:8.

281. Mosenzon O, Wiviott SD, Cahn A, Rozenberg A, Yanuv I, Goodrich EL, et al. Effects of dapagliflozin on development and progression of kidney disease in patients with type 2 diabetes: An analysis from the DECLARE-TIMI 58 randomised trial. Lancet Diabetes Endocrinol 2019;7:606-17.

282. Norhammar A, Bodegård J, Nyström T, Thuresson M, Nathanson D, Eriksson JW. Dapagliflozin and cardiovascular mortality and disease outcomes in a population with type 2 diabetes similar to that of the DECLARE-TIMI 58 trial: A nationwide observational study. Diabetes Obes Metab 2019;21:1136-45.

283. Giles TD, Miller AB, Elkayam U, Bhattacharya M, Perez A. Pioglitazone and heart failure: Results from a controlled study in patients with type 2 diabetes mellitus and systolic dysfunction. J Card Fail 2008; 14:445-52.

284. Marx N, Rosenstock J, Kahn SE, Zinman B, Kastelein JJ, Lachin JM, et al. Design and baseline characteristics of the CARdiovascular outcome Trial of LINAgliptin versus glimepiride in type 2 diabetes 
(CAROLINA®). Diab Vasc Dis Res 2015;12:164-74.

285. Patel A, ADVANCE Collaborative Group, MacMahon S, Chalmers J, Neal B, Woodward M, et al. Effects of a fixed combination of perindopril and indapamide on macrovascular and microvascular outcomes in patients with type 2 diabetes mellitus (the ADVANCE trial): A randomised controlled trial. Lancet 2007;370:829-40.

286. Bolen S, Tseng E, Hutfless S. AHRQ comparative effectiveness reviews. Diabetes Medications for Adults with type 2 Diabetes: An Update. Rockville (MD): Agency for Healthcare Research and Quality (US); 2016.

287. Gallwitz B. The future of combination therapies of insulin with a glucagon-like peptide-1 receptor agonists in type 2 diabetes - Is it advantageous? Eur Endocrinol 2014;10:98-9.

288. Thomsen RW, Baggesen LM, Søgaard M, Pedersen L, Nørrelund H, Buhl ES, et al. Early glycaemic control in metformin users receiving their first add-on therapy: A population-based study of 4,734 people with type 2 diabetes. Diabetologia 2015;58:2247-53.

289. Phung OJ, Scholle JM, Talwar M, Coleman CI. Effect of noninsulin antidiabetic drugs added to metformin therapy on glycemic control, weight gain, and hypoglycemia in type 2 diabetes. JAMA 2010;303:1410-8

290. Salvo F, Moore N, Arnaud M, Robinson P, Raschi E, de Ponti F, et al. Addition of dipeptidyl peptidase-4 inhibitors to sulphonylureas and risk of hypoglycaemia: Systematic review and meta-analysis. BMJ 2016;353:i2231.

291. Nauck MA. Update on developments with SGLT2 inhibitors in the management of type 2 diabetes. Drug Des Devel Ther 2014;8:1335-80.

292. McCulloch DK. Management of Persistent Hyperglycemia in Type 2 Diabetes Mellitus; 2012. p. 5. Available from: http://wwwuptodatecom/ contents/management-of-persistent-hyperglycemia-in-type-2diabetes-mellitus.

293. American Diabetes Association. 4. Comprehensive medical evaluation and assessment of comorbidities: Standards of medical care in diabetes-2019. Diabetes Care 2019;42:S34-45.

294. Chawla A, Chawla R, Jaggi S. Microvasular and macrovascular complications in diabetes mellitus: Distinct or continuum? Indian J Endocrinol Metab 2016;20:546-51.

295. Diabetes Control and Complications Trial Research Group, Nathan DM, Genuth S, Lachin J, Cleary P, Crofford O, et al. The effect of intensive treatment of diabetes on the development and progression of long-term complications in insulin-dependent diabetes mellitus. $\mathrm{N}$ Engl J Med 1993;329:977-86.

296. Ohkubo Y, Kishikawa H, Araki E, Miyata T, Isami S, Motoyoshi $\mathrm{S}$, et al. Intensive insulin therapy prevents the progression of diabetic microvascular complications in Japanese patients with non-insulin-dependent diabetes mellitus: A randomized prospective 6-year study. Diabetes Res Clin Pract 1995;28:103-17.

297. Jindal R, Gupta N, Siddiqui MA. Post-prandial hyperglycaemia. J Indian Academy Clin Med 2013;14:243-

298. Nathan DM, Buse JB, Davidson MB, Heine RJ, Holman RR, Sherwin $\mathrm{R}$, et al. Management of hyperglycemia in type 2 diabetes: A consensus algorithm for the initiation and adjustment of therapy: A consensus statement from the American diabetes association and the European association for the study of diabetes. Diabetes Care 2006;29:1963-72

299. Ceriello A. The glucose triad and its role in comprehensive glycaemic control: Current status, future management. Int $\mathrm{J}$ Clin Pract 2010;64:1705-11

300. Ketema EB, Kibret KT. Correlation of fasting and postprandial plasma glucose with HbAlc in assessing glycemic control; systematic review and meta-analysis. Arch Public Health 2015;73:43.

301. Aravind S, Saboo B, Sadikot S, Shah SN, Makkar B, Kalra S, et al. Consensus statement on management of post-prandial hyperglycemia in clinical practice in India. J Assoc Physicians India 2015;63:45-58.

302. Talib S, Bhattu S, Korpe JS. An observational study on correlation of fasting and postprandial glycemic status to various $\mathrm{HbA1C}$ quintiles in type II diabetics. IOSR J Dent Med Sci 2013;6:14-9.

303. Wang JS, Tu ST, Lee IT, Lin SD, Lin SY, Su SL, et al. Contribution of postprandial glucose to excess hyperglycaemia in Asian type 2 diabetic patients using continuous glucose monitoring. Diabetes Metab Res
Rev 2011;27:79-84.

304. Monnier L, Lapinski H, Colette C. Contributions of fasting and postprandial plasma glucose increments to the overall diurnal hyperglycemia of type 2 diabetic patients: Variations with increasing levels of $\mathrm{HbA}(1 \mathrm{c})$. Diabetes Care 2003;26:881-5.

305. Dickinson S, Colagiuri S, Faramus E, Petocz P, Brand-Miller JC. Postprandial hyperglycemia and insulin sensitivity differ among lean young adults of different ethnicities. J Nutr 2002;132:2574-9.

306. Tan WS, Tan SY, Henry CJ. Ethnic variability in glycemic response to sucrose and isomaltulose. Nutrients 2017;9:347. doi: 10.3390/ nu9040347.

307. Singh SK. Post-prandial hyperglycemia. Indian J Endocrinol Metab 2012;16:S245-7.

308. Ogama N, Sakurai T, Kawashima S, Tanikawa T, Tokuda H, Satake S, et al. Postprandial hyperglycemia is associated with white matter hyperintensity and brain atrophy in older patients with type 2 diabetes mellitus. Front Aging Neurosci 2018;10:273.

309. Sudhir R, Mohan V. Postprandial hyperglycemia in patients with type 2 diabetes mellitus. Treat Endocrinol 2002;1:105-16.

310. Abdul-Ghani MA, Williams K, DeFronzo RA, Stern M. What is the best predictor of future type 2 diabetes? Diabetes Care 2007;30:1544-8.

311. Oh TJ, Lim S, Kim KM, Moon JH, Choi SH, Cho YM, et al. One-hour postload plasma glucose concentration in people with normal glucose homeostasis predicts future diabetes mellitus: A 12-year community-based cohort study. Clin Endocrinol (Oxf) 2017;86:513-9.

312. Jagannathan R, Sevick MA, Li H, Fink D, Dankner R, Chetrit A, et al. Elevated 1-hour plasma glucose levels are associated with dysglycemia, impaired beta-cell function, and insulin sensitivity: A pilot study from a real world health care setting. Endocrine 2016;52:172-5.

313. O'Leary DH, Polak JF, Kronmal RA, Manolio TA, Burke GL, Wolfson SK Jr. Carotid-artery intima and media thickness as a risk factor for myocardial infarction and stroke in older adults. Cardiovascular Health Study Collaborative Research Group. N Engl J Med 1999;340:14-22.

314. Sciacqua A, Miceli S, Carullo G, Greco L, Succurro E, Arturi F, et al. One-hour postload plasma glucose levels and left ventricular mass in hypertensive patients. Diabetes Care 2011;34:1406-11.

315. Wu X, Chen H, Wang Y, Li H. The relationship between coronary risk factors and elevated 1-h postload plasma glucose levels in patients with established coronary heart disease. Clin Endocrinol (Oxf) 2013;78:67-72.

316. Fiorentino TV, Sesti F, Andreozzi F, Pedace E, Sciacqua A, Hribal ML, et al. One-hour post-load hyperglycemia combined with $\mathrm{HbAlc}$ identifies pre-diabetic individuals with a higher cardio-metabolic risk burden. Atherosclerosis 2016;253:61-9.

317. Mohan V, Vijayaprabha R, Rema M. Vascular complications in long-term South Indian NIDDM of over 25 years' duration. Diabetes Res Clin Pract 1996;31:133-40.

318. Marathe PH, Gao HX, Close KL. American diabetes association standards of medical care in diabetes 2017. J Diabetes 2017;9:320-4.

319. Ceriello A. Postprandial Hyperglycemia and Cardiovascular Disease: Is the HEART2D Study the Answer? American Diabetes Association; 2009

320. NAVIGATOR Study Group, Holman RR, Haffner SM, McMurray JJ, Bethel MA, Holzhauer B, et al. Effect of nateglinide on the incidence of diabetes and cardiovascular events. N Engl J Med 2010;362:1463-76.

321. Raz I, Wilson PW, Strojek K, Kowalska I, Bozikov V, Gitt AK, et al. Effects of prandial versus fasting glycemia on cardiovascular outcomes in type 2 diabetes: The HEART2D trial. Diabetes Care 2009;32:381-6.

322. Chiasson JL, Josse RG, Gomis R, Hanefeld M, Karasik A, Laakso M, et al. Acarbose for prevention of type 2 diabetes mellitus: The STOP-NIDDM randomised trial. Lancet 2002;359:2072-7.

323. Holman RR, Coleman RL, Chan JC, Chiasson JL, Feng H, Ge J, et al. Effects of acarbose on cardiovascular and diabetes outcomes in patients with coronary heart disease and impaired glucose tolerance (ACE): A randomised, double-blind, placebo-controlled trial. Lancet Diabetes Endocrinol 2017;5:877-86.

324. Qiao Q, Hu G, Tuomilehto J, Nakagami T, Balkau B, Borch-Johnsen K, et al. Age- and sex-specific prevalence of diabetes and impaired glucose regulation in 11 Asian cohorts. Diabetes Care 2003;26:1770-80. 
325. Nakagami T; DECODA Study Group. Hyperglycaemia and mortality from all causes and from cardiovascular disease in five populations of Asian origin. Diabetologia 2004;47:385-94.

326. Sacks DB. A1C versus glucose testing: A comparison. Diabetes Care 2011;34:518-23.

327. Sheu WH, Rosman A, Mithal A, Chung N, Lim YT, Deerochanawong C, et al. Addressing the burden of type 2 diabetes and cardiovascular disease through the management of postprandial hyperglycaemia: An Asian-pacific perspective and expert recommendations. Diabetes Res Clin Pract 2011;92:312-21.

328. Reynolds AN, Mann JI, Williams S, Venn BJ. Advice to walk after meals is more effective for lowering postprandial glycaemia in type 2 diabetes mellitus than advice that does not specify timing: A randomised crossover study. Diabetologia 2016;59:2572-8.

329. Joshi SR. Post-prandial carbohydrate modulation via gut - Indian perspective. J Assoc Physicians India 2010;58:665.

330. Augustin LS, Kendall CW, Jenkins DJ, Willett WC, Astrup A, Barclay AW, et al. Glycemic index, glycemic load and glycemic response: An International scientific consensus summit from the international carbohydrate quality consortium (ICQC). Nutr Metab Cardiovasc Dis 2015;25:795-815.

331. Ghosh J. Trial of low glycemic diet and acarbose therapy for control of post-prandial hyperglycemia in type 2 diabetes mellitus, preliminary report. Int J Diab Dev Ctries [Internet]. 2005;25(3). Available from: http://www.diabetes.org.in/journal/2005_july-sept/original_article3. pdf. [Last accessed on 2013 Jul 02].

332. Durán A, Martín P, Runkle I, Pérez N, Abad R, Fernández M, et al. Benefits of self-monitoring blood glucose in the management of new-onset type 2 diabetes mellitus: The St Carlos study, a prospective randomized clinic-based interventional study with parallel groups. J Diabetes 2010;2:203-11.

333. Franciosi M, Lucisano G, Pellegrini F, Cantarello A, Consoli A, Cucco L, et al. ROSES: Role of self-monitoring of blood glucose and intensive education in patients with Type 2 diabetes not receiving insulin. A pilot randomized clinical trial. Diabet Med 2011;28:789-96.

334. Polonsky WH, Fisher L, Schikman CH, Hinnen DA, Parkin CG, Jelsovsky Z, et al. Structured self-monitoring of blood glucose significantly reduces $\mathrm{A} 1 \mathrm{C}$ levels in poorly controlled, noninsulin-treated type 2 diabetes: Results from the structured testing program study. Diabetes Care 2011;34:262-7.

335. Mangrola D, Cox C, Furman AS, Krishnan S, Karakas SE. Self blood glucose monitoring underestimates hyperglycemia and hypoglycemia as compared to continuous glucose monitoring in type 1 and type 2 diabetes. Endocr Pract 2018;24:47-52.

336. Kesavadev J, Vigersky R, Shin J, Pillai PB, Shankar A, Sanal G, et al. Assessing the therapeutic utility of professional continuous glucose monitoring in type 2 diabetes across various therapies: A retrospective evaluation. Adv Ther 2017;34:1918-27.

337. Maia FF, Araújo LR. Efficacy of continuous glucose monitoring system (CGMS) to detect postprandial hyperglycemia and unrecognized hypoglycemia in type 1 diabetic patients. Diabetes Res Clin Pract 2007:75:30-4.

338. Kitabchi AE, Umpierrez GE, Miles JM, Fisher JN. Hyperglycemic crises in adult patients with diabetes. Diabetes Care 2009;32:1335-43.

339. Basu A, Close CF, Jenkins D, Krentz AJ, Nattrass M, Wright AD. Persisting mortality in diabetic ketoacidosis. Diabet Med 1993;10:282-4.

340. Malone ML, Gennis V, Goodwin JS. Characteristics of diabetic ketoacidosis in older versus younger adults. J Am Geriatr Soc 1992;40:1100-4.

341. Bhowmick SK, Levens KL, Rettig KR. Hyperosmolar hyperglycemic crisis: An acute life-threatening event in children and adolescents with type 2 diabetes mellitus. Endocr Pract 2005;11:23-9.

342. Fadini GP, de Kreutzenberg SV, Rigato M, Brocco S, Marchesan M, Tiengo A, et al. Characteristics and outcomes of the hyperglycemic hyperosmolar non-ketotic syndrome in a cohort of 51 consecutive cases at a single center. Diabetes Res Clin Pract 2011;94:172-9.

343. Pasquel FJ, Umpierrez GE. Hyperosmolar hyperglycemic state: A historic review of the clinical presentation, diagnosis, and treatment. Diabetes Care 2014;37:3124-31.

344. Kitabchi AE, Nyenwe EA. Hyperglycemic crises in diabetes mellitus:
Diabetic ketoacidosis and hyperglycemic hyperosmolar state. Endocrinol Metab Clin North Am 2006;35:725-51, viii.

345. Indulekha K, Surendar J, Anjana RM, Geetha L, Gokulakrishnan K, Pradeepa R, et al. Metabolic obesity, adipocytokines, and inflammatory markers in Asian Indians-CURES-124. Diabetes Technol Ther 2015;17:134-41.

346. International Hypoglycaemia Study Group. Glucose concentrations of less than $3.0 \mathrm{mmol} / \mathrm{L}(54 \mathrm{mg} / \mathrm{dL})$ should be reported in clinical trials: A joint position statement of the American diabetes association and the European association for the study of diabetes. Diabetes Care 2017;40:155-7.

347. Shriraam V, Mahadevan S, Anitharani M, Jagadeesh NS, Kurup SB, Vidya TA, et al. Reported hypoglycemia in type 2 diabetes mellitus patients: Prevalence and practices-a hospital-based study. Indian J Endocrinol Metab 2017;21:148-53.

348. Vikas Pv, Chandrakumar A, Dilip C, Suriyaprakash TN, Thomas L, Surendran R. Incidence and risk factors of hypoglycemia among Type 2 diabetic patients in a South Indian hospital. Diabetes Metab Syndr 2016;10:S22-5.

349. Kalra S, Mukherjee JJ, Venkataraman S, Bantwal G, Shaikh S, Saboo B, et al. Hypoglycemia: The neglected complication. Indian J Endocrinol Metab 2013;17:819-34.

350. ACCORD Study Group, Cushman WC, Evans GW, Byington RP, Goff DC Jr., Grimm RH Jr., et al. Effects of intensive blood-pressure control in type 2 diabetes mellitus. N Engl J Med 2010;362:1575-85

351. McCoy RG, van Houten HK, Ziegenfuss JY, Shah ND, Wermers RA, Smith SA. Increased mortality of patients with diabetes reporting severe hypoglycemia. Diabetes Care 2012;35:1897-901.

352. Zoungas S, Patel A, Chalmers J, de Galan BE, Li Q, Billot L, et al. Severe hypoglycemia and risks of vascular events and death. N Engl J Med 2010;363:1410-8.

353. Singh AK, Talwalkar PG, Ahluwalia A, Modi KD, Saboo B. Expert group recommendations on detection and management of hypoglycemia in routine clinical practice in insulin treated patients with diabetes. J Assoc Physicians India 2018;66:90-7.

354. Gabriely I, Shamoon H. Hypoglycemia in diabetes: Common, often unrecognized. Cleve Clin J Med 2004;71:335-42.

355. Hirsch IB. Hypoglycemia and the hypoglycemic unawareness syndrome. Diabetes Technol Ther 2000;2 Suppl 1:S81-7.

356. Shafiee G, Mohajeri-Tehrani M, Pajouhi M, Larijani B. The importance of hypoglycemia in diabetic patients. J Diabetes Metab Disord 2012;11:17.

357. Ahmedani MY, Haque MS, Basit A, Fawwad A, Alvi SF. Ramadan prospective diabetes study: The role of drug dosage and timing alteration, active glucose monitoring and patient education. Diabet Med 2012;29:709-15.

358. Bravis V, Hui E, Salih S, Mehar S, Hassanein M, Devendra D. Ramadan education and awareness in diabetes (READ) programme for Muslims with Type 2 diabetes who fast during Ramadan. Diabet Med 2010;27:327-31.

359. Norris SL, Lau J, Smith SJ, Schmid CH, Engelgau MM. Self-management education for adults with type 2 diabetes: A meta-analysis of the effect on glycemic control. Diabetes Care 2002;25:1159-71.

360. Tenzer-Iglesias P, Shannon MH. Managing hypoglycemia in primary care. J Fam Pract 2012;61:S1-8.

361. Noh RM, Graveling AJ, Frier BM. Medically minimising the impact of hypoglycaemia in type 2 diabetes: A review. Expert Opin Pharmacother 2011;12:2161-75.

362. UK Hypoglycaemia Study Group. Risk of hypoglycaemia in types 1 and 2 diabetes: Effects of treatment modalities and their duration. Diabetologia 2007;50:1140-7.

363. Brunton S. Safety and Effectiveness of Modern Insulin Therapy. Current Issues in the Management of Type 2 Diabetes Care. 2009. p. 13.

364. Garber AJ, Clauson P, Pedersen CB, Kølendorf K. Lower risk of hypoglycemia with insulin detemir than with neutral protamine hagedorn insulin in older persons with type 2 diabetes: A pooled analysis of phase III trials. J Am Geriatr Soc 2007;55:1735-40. 
365. Fakhoury W, Lockhart I, Kotchie RW, Aagren M, LeReun C. Indirect comparison of once daily insulin detemir and glargine in reducing weight gain and hypoglycaemic episodes when administered in addition to conventional oral anti-diabetic therapy in patients with type-2 diabetes. Pharmacology 2008;82:156-63.

366. Chan SP, Colagiuri S. Systematic review and meta-analysis of the efficacy and hypoglycemic safety of gliclazide versus other insulinotropic agents. Diabetes Res Clin Pract 2015;110:75-81.

367. Draeger KE, Wernicke-Panten K, Lomp HJ, Schüler E, Rosskamp R. Long-term treatment of type 2 diabetic patients with the new oral antidiabetic agent glimepiride (Amaryl): A double-blind comparison with glibenclamide. Horm Metab Res 1996;28:419-25.

368. Viswanathan M, Joshi SR, Bhansali A. Hypoglycemia in type 2 diabetes: Standpoint of an experts' committee (India hypoglycemia study group). Indian J Endocrinol Metab 2012;16:894-8.

369. Gadkari SS, Maskati QB, Nayak BK. Prevalence of diabetic retinopathy in India: The all India ophthalmological society diabetic retinopathy eye screening study 2014. Indian J Ophthalmol 2016;64:38-44.

370. Patil S, Gogate P, Vora S, Ainapure S, Hingane RN, Kulkarni AN, et al. Prevalence, causes of blindness, visual impairment and cataract surgical services in Sindhudurg district on the western coastal strip of India. Indian J Ophthalmol 2014;62:240-5.

371. American Diabetes Association. 11. Microvascular complications and foot care: Standards of medical care in diabetes-2019. Diabetes Care 2019;42:S124-38.

372. Yau JW, Rogers SL, Kawasaki R, Lamoureux EL, Kowalski JW, Bek T, et al. Global prevalence and major risk factors of diabetic retinopathy. Diabetes Care 2012;35:556-64.

373. Sosale A, Prasanna Kumar KM, Sadikot SM, Nigam A, Bajaj S, Zargar AH, et al. Chronic complications in newly diagnosed patients with Type 2 diabetes mellitus in India. Indian J Endocrinol Metab 2014;18:355-60.

374. Sosale B, Sosale AR, Mohan AR, Kumar PM, Saboo B, Kandula S. Cardiovascular risk factors, micro and macrovascular complications at diagnosis in patients with young onset type 2 diabetes in India: CINDI 2. Indian J Endocrinol Metab 2016;20:114-8.

375. Rani PK, Raman R, Agarwal S, Paul PG, Uthra S, Margabandhu G, et al. Diabetic retinopathy screening model for rural population: Awareness and screening methodology. Rural Remote Health 2005;5:350.

376. Chakrabarti R, Chatterjee T. Tip of the iceberg: The need for diabetic retinopathy screening in developing countries. Lessons from Vietnam. Asia Pac J Ophthalmol (Phila) 2013;2:76-8.

377. Unnikrishnan AG, Kalra S, Tandon N. Diabetic retinopathy care in India: An endocrinology perspective. Indian J Endocrinol Metab 2016;20:S1-2.

378. Kollias AN, Ulbig MW. Diabetic retinopathy: Early diagnosis and effective treatment. Dtsch Arztebl Int 2010;107:75-83.

379. Hammes HP. Optimal treatment of diabetic retinopathy. Ther Adv Endocrinol Metab 2013;4:61-71.

380. American Diabetes Association. Standards of medical care in diabetes-2015 abridged for primary care providers. Clin Diabetes 2015;33:97-111.

381. Garg S, Davis RM. Diabetic retinopathy screening update. Clin Diabetes 2009;27:140-5.

382. Morrison JL, Hodgson LA, Lim LL, Al-Qureshi S. Diabetic retinopathy in pregnancy: A review. Clin Exp Ophthalmol 2016;44:321-34.

383. Scarpa G, Urban F, Vujosevic S, Tessarin M, Gallo G, Visentin A, et al The nonmydriatic fundus camera in diabetic retinopathy screening: A cost-effective study with evaluation for future large-scale application. J Ophthalmol 2016;2016:4625096.

384. Egan AM, McVicker L, Heerey A, Carmody L, Harney F, Dunne FP. Diabetic retinopathy in pregnancy: A population-based study of women with pregestational diabetes. J Diabetes Res 2015;2015:310239.

385. Mallika P, Tan A, SA, TA, Alwi SS, Intan G. Diabetic retinopathy and the effect of pregnancy. Malays Fam Physician 2010;5:2-5.

386. Lauszus F, Klebe JG, Bek T. Diabetic retinopathy in pregnancy during tight metabolic control. Acta Obstet Gynecol Scand 2000;79:367-70.

387. Chen Z, Lin X, Qu B, Gao W, Zuo Y, Peng W, et al. Preoperative expectations and postoperative outcomes of visual functioning among cataract patients in Urban Southern China. PLoS One 2017;12:e0169844.

388. Raymond NT, Varadhan L, Reynold DR, Bush K, Sankaranarayanan S, Bellary S, et al. Higher prevalence of retinopathy in diabetic patients of South Asian ethnicity compared with white Europeans in the community: A cross-sectional study. Diabetes Care 2009;32:410-5.

389. Rema M, Premkumar S, Anitha B, Deepa R, Pradeepa R, Mohan V. Prevalence of diabetic retinopathy in urban India: The Chennai Urban Rural Epidemiology Study (CURES) eye study, I. Invest Ophthalmol Vis Sci 2005;46:2328-33.

390. Ramavat PR, Ramavat MR, Ghugare BW, Vaishnav RG, Joshi MU. Prevalence of diabetic retinopathy in Western Indian type 2 diabetic population: A hospital - Based cross - Sectional study. J Clin Diagn Res 2013;7:1387-90.

391. Raman R, Ganesan S, Pal SS, Kulothungan V, Sharma T. Prevalence and risk factors for diabetic retinopathy in rural India. Sankara Nethralaya diabetic retinopathy epidemiology and molecular genetic study III (SN-DREAMS III), report no 2. BMJ Open Diabetes Res Care 2014;2:e00005.

392. Jotheeswaran AT, Lovakanth N, Nadiga S, Anchala R, Murthy GV, Gilbert CE. Estimating the proportion of persons with diabetes developing diabetic retinopathy in India: A systematic review and meta-analysis. Indian J Endocrinol Metab 2016;20:S51-8.

393. Pradeepa R, Anitha B, Mohan V, Ganesan A, Rema M. Risk factors for diabetic retinopathy in a South Indian Type 2 diabetic population - The Chennai urban rural epidemiology study (CURES) eye study 4. Diabet Med 2008;25:536-42.

394. Shah A, Kanaya AM. Diabetes and associated complications in the South Asian population. Curr Cardiol Rep 2014;16:476.

395. Pradeepa R, Anjana RM, Unnikrishnan R, Ganesan A, Mohan V, Rema M. Risk factors for microvascular complications of diabetes among South Indian subjects with type 2 diabetes-the Chennai Urban Rural Epidemiology Study (CURES) Eye Study-5. Diabetes Technol Ther 2010;12:755-61.

396. Chawla A, Chawla R, editors. Correlation between Retinopathy Microalbuminuria and Other Modifiable Risk Factors. Presented on American Diabetes Association's 75 ${ }^{\text {th }}$ Scientific Session; 2015.

397. Chawla A, Chawla R, Jaggi S, editors. Trained nurse Operated Telemedicine Based Retinal Examination-A Novel Cost Effective Model for the Developing World. Endocrine Practice; 2016.

398. Eszes DJ, Szabó DJ, Russell G, Kirby P, Paulik E, Nagymajtényi L, et al. Diabetic retinopathy screening using telemedicine tools: Pilot study in Hungary. J Diabetes Res 2016;2016:4529824.

399. Bayram EH, Sezer AD, Elçioğlu HK. Diabetic neuropathy and treatment strategy-new challenges and applications. In: Smart Drug Delivery System. 2016. p. 373.

400. Singh R, Kishore L, Kaur N. Diabetic peripheral neuropathy: Current perspective and future directions. Pharmacol Res 2014;80:21-35.

401. Snyder MJ, Gibbs LM, Lindsay TJ. Treating painful diabetic peripheral neuropathy: An update. Am Fam Physician 2016;94:227-34.

402. Gill HK, Yadav SB, Ramesh V, Bhatia E. A prospective study of prevalence and association of peripheral neuropathy in Indian patients with newly diagnosed type 2 diabetes mellitus. J Postgrad Med 2014;60:270-5.

403. Rani PK, Raman R, Rachapalli SR, Pal SS, Kulothungan V, Sharma T. Prevalence and risk factors for severity of diabetic neuropathy in type 2 diabetes mellitus. Indian J Med Sci 2010;64:51-7.

404. Juster-Switlyk K, Smith AG. Updates in diabetic peripheral neuropathy. F1000Res 2016;5. pii: F1000.

405. Kakrani AL, Gokhale VS, Vohra KV, Chaudhary N. Clinical and nerve conduction study correlation in patients of diabetic neuropathy. J Assoc Physicians India 2014;62:24-7.

406. Bansal D, Gudala K, Muthyala H, Esam HP, Nayakallu R, Bhansali A. Prevalence and risk factors of development of peripheral diabetic neuropathy in type 2 diabetes mellitus in a tertiary care setting. J Diabetes Investig 2014;5:714-21.

407. Kannan MA, Sarva S, Kandadai RM, Paturi VR, Jabeen SA, Borgohain R. Prevalence of neuropathy in patients with impaired glucose tolerance using various electrophysiological tests. Neurol India 2014;62:656-61.

408. Meijer JW, Smit AJ, Sonderen EV, Groothoff JW, Eisma WH, 
Links TP. Symptom scoring systems to diagnose distal polyneuropathy in diabetes: The diabetic neuropathy symptom score. Diabet Med 2002; 19:962-5.

409. Boulton AJ. Management of diabetic peripheral neuropathy. Clin Diabetes 2005;23:9-15.

410. Chawla A, Bhasin G, Chawla R. Validation of neuropathy symptoms score (NSS) and neuropathy disability score (NDS) in the clinical diagnosis of peripheral neuropathy in middle aged people with diabetes. Int J Family Practice 2013;12:1.

411. Hussain G, Rizvi SA, Singhal S, Zubair M, Ahmad J. Cross sectional study to evaluate the effect of duration of type 2 diabetes mellitus on the nerve conduction velocity in diabetic peripheral neuropathy. Diabetes Metab Syndr 2014;8:48-52.

412. Afifi L, Abdelalim AM, Ashour AS. Correlation between clinical neuropathy scores and nerve conduction studies in patients with diabetic peripheral neuropathy. Egypt J Neurol Psychiatr Neurosurg 2016;53:248.

413. Asad A, Hameed MA, Khan UA, Butt MU, Ahmed N, Nadeem A. Comparison of nerve conduction studies with diabetic neuropathy symptom score and diabetic neuropathy examination score in type- 2 diabetics for detection of sensorimotor polyneuropathy. J Pak Med Assoc 2009;59:594-8.

414. Kamel SR, Hamdy M, Omar HA. Clinical diagnosis of distal diabetic polyneuropathy using neurological examination scores: Correlation with nerve conduction studies. Egypt Rheumatol Rehabil 2015;42: $128-$

415. Backonja M, Beydoun A, Edwards KR, Schwartz SL, Fonseca V, Hes M, et al. Gabapentin for the symptomatic treatment of painful neuropathy in patients with diabetes mellitus: A randomized controlled trial. JAMA 1998;280:1831-6.

416. Javed S, Petropoulos IN, Alam U, Malik RA. Treatment of painful diabetic neuropathy. Ther Adv Chronic Dis 2015;6:15-28.

417. Arezzo JC, Rosenstock J, Lamoreaux L, Pauer L. Efficacy and safety of pregabalin $600 \mathrm{mg} / \mathrm{d}$ for treating painful diabetic peripheral neuropathy: A double-blind placebo-controlled trial. BMC Neurol 2008;8:33.

418. Richter RW, Portenoy R, Sharma U, Lamoreaux L, Bockbrader H, Knapp LE. Relief of painful diabetic peripheral neuropathy with pregabalin: A randomized, placebo-controlled trial. J Pain 2005;6:253-60.

419. Wang D, Bao JB, Zhang K. Pregabalin for the treatment of neuropathic pain in adults: A systematic review of randomized controlled trials. Int J Clin Exp Med 2017;10:16-29.

420. Roy MK, Kuriakose AS, Varma SK, Jacob LA, Beegum NJ. A study on comparative efficacy and cost effectiveness of pregabalin and duloxetine used in diabetic neuropathic pain. Diabetes Metab Syndr 2017;11:31-5.

421. Ormseth MJ, Scholz BA, Boomershine CS. Duloxetine in the management of diabetic peripheral neuropathic pain. Patient Prefer Adherence 2011;5:343-56.

422. Smith T, Nicholson RA. Review of duloxetine in the management of diabetic peripheral neuropathic pain. Vasc Health Risk Manag 2007:3:833-44.

423. Goldstein DJ, Lu Y, Detke MJ, Lee TC, Iyengar S. Duloxetine vs. placebo in patients with painful diabetic neuropathy. Pain 2005;116:109-18.

424. Raskin J, Pritchett YL, Wang F, D’Souza DN, Waninger AL, Iyengar $\mathrm{S}$, et al. A double-blind, randomized multicenter trial comparing duloxetine with placebo in the management of diabetic peripheral neuropathic pain. Pain Med 2005;6:346-56.

425. Wernicke JF, Pritchett YL, D'Souza DN, Waninger A, Tran P, Iyengar S, et al. A randomized controlled trial of duloxetine in diabetic peripheral neuropathic pain. Neurology 2006;67:1411-20.

426. Lunn MP, Hughes RA, Wiffen PJ. Duloxetine for treating painful neuropathy, chronic pain or fibromyalgia. Cochrane Database Syst Rev 2014:CD007115.

427. Christoph T, Schröder W, Tallarida RJ, de Vry J, Tzschentke TM. Spinal-supraspinal and intrinsic $\mu$-opioid receptor agonist-norepinephrine reuptake inhibitor (MOR-NRI) synergy of tapentadol in diabetic heat hyperalgesia in mice. J Pharmacol Exp Ther
2013;347:794-801

428. Schwartz S, Etropolski M, Shapiro DY, Okamoto A, Lange R, Haeussler J, et al. Safety and efficacy of tapentadol ER in patients with painful diabetic peripheral neuropathy: Results of a randomized-withdrawal, placebo-controlled trial. Curr Med Res Opin 2011;27:151-62.

429. Vinik AI, Shapiro DY, Rauschkolb C, Lange B, Karcher K, Pennett D, et al. A randomized withdrawal, placebo-controlled study evaluating the efficacy and tolerability of tapentadol extended release in patients with chronic painful diabetic peripheral neuropathy. Diabetes Care 2014;37:2302-9.

430. Duehmke RM, Derry S, Wiffen PJ, Bell RF, Aldington D, Moore RA. Tramadol for neuropathic pain in adults. Cochrane Database Syst Rev 2017;6:CD003726.

431. Morón Merchante I, Pergolizzi JV Jr., van de Laar M, Mellinghoff HU, Nalamachu S, O'Brien J, et al. Tramadol/paracetamol fixed-dose combination for chronic pain management in family practice: A clinical review. ISRN Family Med 2013;2013:638469.

432. Sadosky A, Schaefer C, Mann R, Bergstrom F, Baik R, Parsons B, et al. Burden of illness associated with painful diabetic peripheral neuropathy among adults seeking treatment in the US: Results from a retrospective chart review and cross-sectional survey. Diabetes Metab Syndr Obes 2013;6:79-92.

433. Finnerup NB, Attal N, Haroutounian S, McNicol E, Baron R, Dworkin RH, et al. Pharmacotherapy for neuropathic pain in adults: A systematic review and meta-analysis. Lancet Neurol 2015;14:162-73.

434. Griebeler ML, Morey-Vargas OL, Brito JP, Tsapas A, Wang Z, Carranza Leon BG, et al. Pharmacologic interventions for painful diabetic neuropathy: An umbrella systematic review and comparative effectiveness network meta-analysis. Ann Intern Med 2014;161:639-49.

435. Saarto T, Wiffen PJ. Antidepressants for neuropathic pain. Cochrane Database Syst Rev 2007:CD005454.

436. Wiffen PJ, Collins S, McQuay HJ, Carroll D, Jadad A, Moore RA. WITHDRAWN. Anticonvulsant drugs for acute and chronic pain. Cochrane Database Syst Rev 2010:CD001133.

437. Schreiber AK, Nones CF, Reis RC, Chichorro JG, Cunha JM. Diabetic neuropathic pain: Physiopathology and treatment. World J Diabetes 2015;6:432-44

438. Herrmann DN, Barbano RL, Hart-Gouleau S, Pennella-Vaughan J, Dworkin RH. An open-label study of the lidocaine patch $5 \%$ in painful idiopathic sensory polyneuropathy. Pain Med 2005;6:379-84.

439. Reidy K, Kang HM, Hostetter T, Susztak K. Molecular mechanisms of diabetic kidney disease. J Clin Invest 2014;124:2333-40.

440. Ahmad J. Management of diabetic nephropathy: Recent progress and future perspective. Diabetes Metab Syndr 2015;9:343-58.

441. Agarwal SK, Srivastava RK. Chronic kidney disease in India: Challenges and solutions. Nephron Clin Pract 2009;111:c197-203.

442. Prasannakumar M, Rajput R, Seshadri K, Talwalkar P, Agarwal P, Gokulnath $\mathrm{G}$, et al. An observational, cross-sectional study to assess the prevalence of chronic kidney disease in type 2 diabetes patients in India (START -India). Indian J Endocrinol Metab 2015;19:520-3.

443. Kher V. End-stage renal disease in developing countries. Kidney Int 2002;62:350-62.

444. Wetmore JB, Collins AJ. Global challenges posed by the growth of end-stage renal disease. Ren Replace Ther 2016;2:15.

445. Cade WT. Diabetes-related microvascular and macrovascular diseases in the physical therapy setting. Phys Ther 2008;88:1322-35.

446. Singh A, Satchell SC. Microalbuminuria: Causes and implications. Pediatr Nephrol 2011;26:1957-65.

447. Lozano-Maneiro L, Puente-García A. Renin-angiotensin-aldosterone system blockade in diabetic nephropathy. Present evidences. J Clin Med 2015;4:1908-37.

448. Vivian E, Mannebach C. Therapeutic approaches to slowing the progression of diabetic nephropathy - Is less best? Drugs Context 2013;2013:212249.

449. Sahay M, Sahay R, Baruah MP. Nutrition in chronic kidney disease. J Med Nutr Nutraceutical 2014;3:11.

450. Rigalleau V, Lasseur C, Raffaitin C, Beauvieux MC, Barthe N, Chauveau $\mathrm{P}$, et al. Normoalbuminuric renal-insufficient diabetic patients: A lower-risk group. Diabetes Care 2007;30:2034-9. 
451. Chawla SPS, Kaur S, Bharti A, Garg R, Kaur M, Soin D, et al. Impact of health education on knowledge, attitude, practices and glycemic control in type 2 diabetes mellitus. J Family Med Prim Care 2019;8:261-8.

452. Becker GJ, Wheeler DC, de Zeeuw D, et al. Kidney disease: Improving global outcomes (KDIGO) blood pressure work group. KDIGO clinical practice guideline for the management of blood pressure in chronic kidney disease. Kidney Int Suppl 2012;2:337-414.

453. KDOQI. KDOQI clinical practice guidelines and clinical practice recommendations for diabetes and chronic kidney disease. Am J Kidney Dis 2007;49:S12-154.

454. Levey AS, Coresh J, Balk E, Kausz AT, Levin A, Steffes MW, et al. National kidney foundation practice guidelines for chronic kidney disease: Evaluation, classification, and stratification. Ann Intern Med 2003;139:137-47.

455. American Diabetes Association. Executive summary: Standards of medical care in diabetes--2011. Diabetes Care 2011;34 Suppl 1:S4-10.

456. Kumar PS, Rao UR, Abhilash T, et al. Evaluation of random urine sample protein: Creatinine ratio as an index of 24 hour urine protein in patients with various renal disorders in tertiary care center. Int J Adv Med 2016;3:855.

457. Viswanathan V, Chamukuttan S, Kuniyil S, Ambady R. Evaluation of a simple, random urine test for prospective analysis of proteinuria in Type 2 diabetes: A six year follow-up study. Diabetes Res Clin Pract 2000;49:143-7.

458. Kondaveeti SB, DK, Mishra S, Kumar RA, Shaker IA. Evaluation of glycated albumin and microalbuminuria as early risk markers of nephropathy in type 2 diabetes mellitus. J Clin Diagn Res 2013;7:1280-3.

459. Jafar TH, Chaturvedi N, Hatcher J, Levey AS. Use of albumin creatinine ratio and urine albumin concentration as a screening test for albuminuria in an Indo-Asian population. Nephrol Dial Transplant 2007;22:2194-200.

460. Heathcote KL, Wilson MP, Quest DW, Wilson TW. Prevalence and duration of exercise induced albuminuria in healthy people. Clin Invest Med 2009;32:E261-5.

461. Sadjadi SA, Jaipaul N. Correlation of random urine protein creatinine (P-C) ratio with 24-hour urine protein and $\mathrm{P}-\mathrm{C}$ ratio, based on physical activity: A pilot study. Ther Clin Risk Manag 2010;6:351-7.

462. Agrawal RP, Ola V, Bishnoi P, Gothwal S, Sirohi P, Agrawal R. Prevalence of micro and macrovascular complications and their risk factors in type-2 diabetes mellitus. J Assoc Physicians India 2014;62:504-8.

463. Jadhav UM, Kadam NN. Association of microalbuminuria with carotid intima-media thickness and coronary artery disease-a cross-sectional study in Western India. J Assoc Physicians India 2002;50:1124-9.

464. Thampy A, Pais CC. Early clinical implications of microalbuminuria in patients with acute ischaemic stroke. J Clin Diagn Res 2016;10:OC29-31.

465. Panchapakesan U, Pollock C. The role of dipeptidyl peptidase -4 inhibitors in diabetic kidney disease. Front Immunol 2015;6:443.

466. Perkovic V, Jardine MJ, Neal B, Bompoint S, Heerspink HJ, Charytan DM, et al. Canagliflozin and renal outcomes in type 2 diabetes and nephropathy. N Engl J Med 2019;380:2295-306.

467. Perkovic V, de Zeeuw D, Mahaffey KW, Fulcher G, Erondu N, Shaw W, et al. Canagliflozin and renal outcomes in type 2 diabetes: Results from the CANVAS Program randomised clinical trials. Lancet Diabetes Endocrinol 2018;6:691-704.

468. Monteiro P, Bergenstal RM, Toural E, Inzucchi SE, Zinman B, Hantel S, et al. Efficacy and safety of empagliflozin in older patients in the EMPA-REG OUTCOME® trial. Age Ageing 2019;48:859-66.

469. Sahay B. Dietary carbohydrate content in Indian diabetic patients. Medicine 2012;22:235-39.

470. Viswanathan V, Snehalatha C, Varadharani M. Prevalence of albuminuria among vegetarian and non-vegetarian south Indian diabetic patients. Indian J Nephrol 2002;12:73-6.

471. Beasley JM, Wylie-Rosett J. The role of dietary proteins among persons with diabetes. Curr Atheroscler Rep 2013;15:348.

472. Hallan SI, Orth SR. Smoking is a risk factor in the progression to kidney failure. Kidney Int 2011;80:516-23.

473. Orth SR. Cigarette smoking: An important renal risk factor - Far beyond carcinogenesis. Tob Induc Dis 2002;1:137-55.

474. Shahid SM, Mahboob T. Cigarette smoking: An environmental risk factor for progression of nephropathy in diabetes. Int J Diabetes Dev Ctries 2007;27:4

475. Elihimas Júnior UF, Elihimas HC, Lemos VM, Leão Mde A, Sá MP, França EE, et al. Smoking as risk factor for chronic kidney disease: Systematic review. J Bras Nefrol 2014;36:519-28.

476. Gupta RK, Gupta R, Maheshwari VD, Mawliya M. Impact of smoking on microalbuminuria and urinary albumin creatinine ratio in non-diabetic normotensive smokers. Indian J Nephrol 2014;24:92-6.

477. Thakkar B, Arora K, Vekariya R, et al. Prevalence of microalbuminuria in newly diagnosed type 2 diabetes mellitus. Natl J Integr Res Med 2011;2:22-5.

478. Varghese A, Deepa R, Rema M, Mohan V. Prevalence of microalbuminuria in type 2 diabetes mellitus at a diabetes centre in southern India. Postgrad Med J 2001;77:399-402.

479. Saha TK, Bhattarai AM, Batra HS, Banerjee M, Misra $P$, Ambade V. Correlation of microalbuminuria with estimated GFR (eGFR) by Cockcroft-Gault and MDRD formula in type 2 diabetics and hypertensives. Indian J Clin Biochem 2015;30:271-4

480. Bonakdaran S, Hami M, Hatefi A. The effects of calcitriol on albuminuria in patients with type-2 diabetes mellitus. Saudi J Kidney Dis Transpl 2012;23:1215-20.

481. Shahi S, Kumar A, Kumar S, et al. Epidemiology of diabetic foot complications in a podiatry clinic of a tertiary hospital in South India. Indian J Health Sci 2015;8:48.

482. Pendsey SP. Understanding diabetic foot. Int J Diabetes Dev Ctries 2010;30:75-9.

483. Ghosh P, Valia R. Burden of diabetic foot ulcers in India: Evidence Landscape from published literature. Value in Health 2017;20:A485.

484. Vijay V, Snehalatha C, Ramachandran A. Sociocultural practices that may affect the development of the diabetic foot. IDF Bulletin 1997;42:10-2.

485. Shobhana R, Rao PR, Lavanya A, Vijay V, Ramachandran A. Foot care economics - cost burden to diabetic patients with foot complications: A study from southern India. J Assoc Physicians India 2001;49:530-3.

486. Boulton A, Armstrong D, Kirsner RS, et al. Diagnosis and Management of Diabetic Foot Complications. Arlington (VA): American Diabetes Association; 2018.

487. Vijay V, Bamila S, Rani A. In: Banerjee S, editor. RSSDI Hand Book of Diabetes Mellitus. Scientific Book.Com; 2016.

488. Viswanathan V, Thomas N, Tandon N, Asirvatham A, Rajasekar S, Ramachandran A, et al. Profile of diabetic foot complications and its associated complications-a multicentric study from India. J Assoc Physicians India 2005;53:933-6.

489. Morbach S, Lutale JK, Viswanathan V, Möllenberg J, Ochs HR, Rajashekar S, et al. Regional differences in risk factors and clinical presentation of diabetic foot lesions. Diabet Med 2004;21:91-5.

490. Viswanathan V, Kumpatla S. Pattern and causes of amputation in diabetic patients-a multicentric study from India. J Assoc Physicians India 2011;59:148-51.

491. Viswanathan V, Wadud JR, Madhavan S, Rajasekar S, Kumpatla S, Lutale $\mathrm{JK}$, et al. Comparison of post amputation outcome in patients with type 2 diabetes from specialized foot care centres in three developing countries. Diabetes Res Clin Pract 2010;88:146-50.

492. Viswanathan V. The diabetic foot: Perspectives from Chennai, South India. Int J Low Extrem Wounds 2007;6:34-6.

493. Armstrong DG, Boulton AJ, Bus SA. Diabetic foot ulcers and their recurrence. N Engl J Med 2017;376:2367-75.

494. Kucera T, Shaikh HH, Sponer P. Charcot neuropathic arthropathy of the foot: A literature review and single-center experience. J Diabetes Res 2016;2016:3207043.

495. Rogers LC, Frykberg RG, Armstrong DG, Boulton AJ, Edmonds M, Van GH, et al. The Charcot foot in diabetes. Diabetes Care 2011;34:2123-9.

496. Carmeliet P. Mechanisms of angiogenesis and arteriogenesis. Nat Med 2000;6:389-95

497. Lipsky BA, Berendt AR, Deery HG, Embil JM, Joseph WS, 
Karchmer AW, et al. Diagnosis and treatment of diabetic foot infections. Clin Infect Dis 2004;39:885-910.

498. Viswanathan V. An aggressive and multi-pronged approach to prevent amputations in India. Int J Diabetes Dev Ctries 2014;34:123-4.

499. Mills JL Sr., Conte MS, Armstrong DG, Pomposelli FB, Schanzer A, Sidawy AN, et al. The society for vascular surgery lower extremity threatened limb classification system: Risk stratification based on wound, ischemia, and foot infection (WIfI). J Vasc Surg 2014;59:220-340.

500. Viswanathan V, Madhavan S, Rajasekar S, Chamukuttan S, Ambady R. Amputation prevention initiative in South India: Positive impact of foot care education. Diabetes Care 2005;28:1019-21.

501. Foot IWGotD. International Consensus on the Diabetic Foot; 2019. Available from: http://iwgdf.org/. [Last accessed on 2019].

502. Afifi L, Abdelalim A, Ashour A, et al. Correlation between clinical neuropathy scores and nerve conduction studies in patients with diabetic peripheral neuropathy. Egypt J Neurol Psychiatr Neurosurg 2016;53:248

503. Kamel S, Hamdy M, Omar H, et al. Clinical diagnosis of distal diabetic polyneuropathy using neurological examination scores: Correlation with nerve conduction studies. Egypt Rheumatol Rehabil 2015;42:128.

504. Jayaprakash P, Bhansali A, Bhansali S, Dutta P, Anantharaman R, Shanmugasundar G, et al. Validation of bedside methods in evaluation of diabetic peripheral neuropathy. Indian J Med Res 2011;133:645-9.

505. Mittal J, Khurana A, Mahajan D, et al. A comparative study of various bedside methods in detection of diabetic polyneuropathy in type 2 diabetes patients. JMEDS 2013;16:9702-6.

506. Chawla A, Bhasin G, Chawla R. Validation of Neuropathy Symptoms Score (NSS) and Neuropathy Disability Score (NDS) in the clinical diagnosis of peripheral neuropathy in middle aged people with diabetes. Int J Fam Pract 2013;12:1-4.

507. Dixit S, Maiya A. Diabetic peripheral neuropathy and its evaluation in a clinical scenario: A review. J Postgrad Med 2014;60:33-40.

508. Vijay V, Snehalatha C, Seena R, et al. The rydel seiffer tuning fork: An inexpensive device for screening diabetic patients with high-risk foot. Pract Diabetes Int 2001;18:155-6.

509. Viswanathan V, Snehalatha C, Seena R, Ramachandran A. Early recognition of diabetic neuropathy: Evaluation of a simple outpatient procedure using thermal perception. Postgrad Med J 2002;78:541-2.

510. Vijay V, Seena R, Lalitha S, Snehalatha C, Ramachandran A. A simple device for foot pressure measurement. Evaluation in south Indian NIDDM subjects. Diabetes Care 1998;21:1205-6.

511. Gnanasundaram S, Ramalingam P, Das BN, Viswanathan V. Gait changes in persons with diabetes: Early risk marker for diabetic foot ulcer. Foot Ankle Surg 2020;26:163-8.

512. Rajagopalan C, Viswanathan V, Rajsekar SE. Diabetic foot ulcers-comparison of performance of ankle-brachial index and transcutaneous partial oxygen pressure in predicting outcome. Int $\mathrm{J}$ Diabetes Dev Ctries 2018;38:179-84.

513. Abbas Z, Viswanathan V. The diabetic foot in Africa and India. Int Diab Monit 2007;19:8-12.

514. Viswanathan V, Madhavan S, Gnanasundaram S, Gopalakrishna G, Das BN, Rajasekar S, et al. Effectiveness of different types of footwear insoles for the diabetic neuropathic foot: A follow-up study. Diabetes Care 2004;27:474-7.

515. Chandalia HB, Singh D, Kapoor V, Chandalia SH, Lamba PS. Footwear and foot care knowledge as risk factors for foot problems in Indian diabetics. Int J Diabetes Dev Ctries 2008;28:109-13.

516. Lipsky BA, Peters EJ, Senneville E, Berendt AR, Embil JM, Lavery LA, et al. Expert opinion on the management of infections in the diabetic foot. Diabetes Metab Res Rev 2012;28 Suppl 1:163-78.

517. Vijay V, Badrinath $\mathrm{H}$, Snehalatha $\mathrm{C}$, et al. Effect of treatment with the combination of Alpha - Lipoic acid, chromium and inositol on diabetic peripheral neuropathy. Asian J Diabetol 2003;5:9-15.

518. Vijay V, Pendsey S, Sekar N, et al. A phase III study to evaluate the safety and efficacy of recombinant human epidermal growth factor (REGEN - DTM 150) in healing diabetic foot ulcers. Wounds 2006;18:186-96.

519. Grek CL, Prasad GM, Viswanathan V, Armstrong DG, Gourdie RG, Ghatnekar GS. Topical administration of a connexin43-based peptide augments healing of chronic neuropathic diabetic foot ulcers: A multicenter, randomized trial. Wound Repair Regen 2015;23:203-12.

520. Jenifer J, Geethalakshmi S, Satyavani K, et al. Bioburden vs. antibiogram of diabetic foot infection. Clin Res Foot Ankle 1:1000121.

521. Ezhilarasi K, Abirami P, Vijay V. Healing effect of hyperbaric oxygen therapy as an adjunctive treatment on diabetic foot ulcer patients in short duration - A brief report. Asian J Sci and Tech 2018;9:7743-6.

522. Armstrong DG, Lavery LA, Bushman TR. Peak foot pressures influence the healing time of diabetic foot ulcers treated with total contact casts. J Rehabil Res Dev 1998;35:1-5.

523. Yazdanpanah L, Nasiri M, Adarvishi S. Literature review on the management of diabetic foot ulcer. World J Diabetes 2015;6:37-53.

524. Wu SC, Crews RT, Armstrong DG. The pivotal role of offloading in the management of neuropathic foot ulceration. Curr Diab Rep 2005;5:423-9.

525. Kari SV. The economical way to off-load diabetic foot ulcers mandakini off-loading device. Indian J Surg 2010;72:133-4.

526. Agrawal V. Easy ways to offload diabetic foot ulcer in rural setup. Int $\mathrm{J}$ Biomed Adv Res 2014;5:187-9.

527. Shankhdhar K. Improvisation is the key to success: The samadhan system. Adv Skin Wound Care 2006;19:379-83.

528. Liu S, He CZ, Cai YT, Xing QP, Guo YZ, Chen ZL, et al. Evaluation of negative-pressure wound therapy for patients with diabetic foot ulcers: Systematic review and meta-analysis. Ther Clin Risk Manag 2017;13:533-44.

529. Viswanathan V, Kesavan R, Kavitha K, et al. Evaluation of roger's Charcot foot classification system in South Indian diabetic subjects with Charcot foot. J Diabetic Foot Complications 2012;4:67-70.

530. Richardson JK, Ching C, Hurvitz EA. The relationship between electromyographically documented peripheral neuropathy and falls. J Am Geriatr Soc 1992;40:1008-12.

531. Wallace C, Reiber GE, LeMaster J, Smith DG, Sullivan K, Hayes S, et al. Incidence of falls, risk factors for falls, and fall-related fractures in individuals with diabetes and a prior foot ulcer. Diabetes Care 2002;25:1983-6.

532. Oliveira PP, Fachin SM, Tozatti J, Ferreira MC, Marinheiro LP. Comparative analysis of risk for falls in patients with and without type 2 diabetes mellitus. Rev Assoc Med Bras (1992) 2012;58:234-9.

533. Martín-Timón I, Sevillano-Collantes C, Segura-Galindo A, Del Cañizo-Gómez FJ. Type 2 diabetes and cardiovascular disease: Have all risk factors the same strength? World J Diabetes 2014;5:444-70

534. American Diabetes Association. 8. Cardiovascular Disease and Risk Management. Diabetes Care 2016;39 Suppl 1:S60-71.

535. Prabhakaran D, Jeemon P, Roy A. Cardiovascular diseases in India: Current epidemiology and future directions. Circulation 2016;133:1605-20.

536. Gupta A, Gupta R, Sharma KK, Lodha S, Achari V, Asirvatham AJ, et al. Prevalence of diabetes and cardiovascular risk factors in middle-class urban participants in India. BMJ Open Diabetes Res Care 2014;2: 000048 .

537. O’Donnell MJ, Chin SL, Rangarajan S, Xavier D, Liu L, Zhang H, et al. Global and regional effects of potentially modifiable risk factors associated with acute stroke in 32 countries (INTERSTROKE): A case-control study. Lancet 2016;388:761-75.

538. Yusuf S, Hawken S, Ounpuu S, Dans T, Avezum A, Lanas F, et al. Effect of potentially modifiable risk factors associated with myocardial infarction in 52 countries (the INTERHEART study): Case-control study. Lancet 2004;364:937-52.

539. Gant CM, Mensink I, Binnenmars SH, van der Palen JA, Bakker SJ, Navis G, et al. Body weight course in the DIAbetes and Lifestyle cohort twente (DIALECT-1)-A 20-year observational study. PLoS One 2019;14:e218400

540. Mortada I. Hyperuricemia, type 2 diabetes mellitus, and hypertension: An emerging association. Curr Hypertens Rep 2017;19:69.

541. Hippisley-Cox J, Coupland C, Brindle P. Development and validation of QRISK3 risk prediction algorithms to estimate future risk of cardiovascular disease: Prospective cohort study. BMJ 2017;357:j2099.

542. Robson J, Hippisley-Cox J, Coupland C. QRISK or Framingham? Br J Clin Pharmacol 2012;74:545-6. 
543. Stevens RJ, Kothari V, Adler AI, Stratton IM; United Kingdom Prospective Diabetes Study (UKPDS) Group. The UKPDS risk engine: A model for the risk of coronary heart disease in Type II diabetes (UKPDS 56). Clin Sci (Lond) 2001;101:671-9.

544. Khunti K, Ji L, Medina J, Surmont F, Kosiborod M. Type 2 diabetes treatment and outcomes worldwide: A short review of the DISCOVER study programme. Diabetes Obes Metab 2019;21:2349-53.

545. Chandra KS, Bansal M, Nair T, Iyengar SS, Gupta R, Manchanda SC, et al. Consensus statement on management of dyslipidemia in Indian subjects. Indian Heart J 2014;66 Suppl 3:S1-51.

546. Authors/Task Force Members, Piepoli MF, Hoes AW, Agewall S, Albus C, Brotons C, et al. 2016 European guidelines on cardiovascular disease prevention in clinical practice: The sixth joint task force of the European society of cardiology and other societies on cardiovascular disease prevention in clinical practice (constituted by representatives of 10 societies and by invited experts): Developed with the special contribution of the European association for cardiovascular prevention \& rehabilitation (EACPR). Eur J Prev Cardiol 2016;23:NP1-96.

547. Dehghan M, Mente A, Zhang X, Swaminathan S, Li W, Mohan V, et al. Associations of fats and carbohydrate intake with cardiovascular disease and mortality in 18 countries from five continents (PURE): A prospective cohort study. Lancet 2017;390:2050-62.

548. Gray. Nutritional Recommendations for Individuals with Diabetes South Endotext. Dartmouth (MA): MDText.com, Inc. https://www. ncbi.nlm.nih.gov/books/NBK279012/. [Last updated 2015 May 31].

549. Varghese T, Schultz WM, McCue AA, Lambert CT, Sandesara PB, Eapen DJ, et al. Physical activity in the prevention of coronary heart disease: Implications for the clinician. Heart 2016;102:904-9.

550. Buttar HS, Li T, Ravi N. Prevention of cardiovascular diseases: Role of exercise, dietary interventions, obesity and smoking cessation. Exp Clin Cardiol 2005;10:229-49.

551. Lanier JB, Bury DC, Richardson SW. Diet and physical activity for cardiovascular disease prevention. Am Fam Physician 2016;93:919-24.

552. Shantakumari N, Sequeira S, El deeb R. Effects of a yoga intervention on lipid profiles of diabetes patients with dyslipidemia. Indian Heart $\mathrm{J}$ 2013;65:127-31.

553. Cui J, Yan JH, Yan LM, Pan L, Le JJ, Guo YZ. Effects of yoga in adults with type 2 diabetes mellitus: A meta-analysis. J Diabetes Investig 2017;8:201-9.

554. Innes KE, Selfe TK. Yoga for adults with type 2 diabetes: A systematic review of controlled trials. J Diabetes Res 2016;2016:6979370.

555. Chimkode SM, Kumaran SD, Kanhere VV, Shivanna R. Effect of yoga on blood glucose levels in patients with type 2 diabetes mellitus. J Clin Diagn Res 2015;9:CC01-3.

556. Steptoe A, Kivimäki M. Stress and cardiovascular disease. Nat Rev Cardiol 2012;9:360-70.

557. Gulliksson M, Burell G, Vessby B, Lundin L, Toss H, Svärdsudd K. Randomized controlled trial of cognitive behavioral therapy vs. standard treatment to prevent recurrent cardiovascular events in patients with coronary heart disease: Secondary Prevention in Uppsala Primary Health Care project (SUPRIM). Arch Intern Med 2011;171:134-40.

558. Richards SH, Anderson L, Jenkinson CE, Whalley B, Rees K, Davies P, et al. Psychological interventions for coronary heart disease. Cochrane Database Syst Rev 2017;4:CD002902.

559. Malik P, Dwivedi S. Diabetes and cardiovascular diseases. JIMSA 2015;28:61-3

560. ASCEND Study Collaborative Group, Bowman L, Mafham M, Wallendszus K, Stevens W, Buck G, et al. Effects of Aspirin for Primary Prevention in Persons with Diabetes Mellitus. N Engl J Med 2018;379:1529-39.

561. Xie M, Shan Z, Zhang Y, Chen S, Yang W, Bao W, et al. Aspirin for primary prevention of cardiovascular events: Meta-analysis of randomized controlled trials and subgroup analysis by sex and diabetes status. PLoS One 2014;9:e90286.

562. JM, CV, CA. Aspirin for the Primary Prevention of Cardiovascular Events: A Systematic Evidence Review for the U.S. Preventive Services Task Force. Rockville (MD): Agency for Healthcare Research and Quality (US); 2015. Available from: http://www.ncbi.nlm.nih.gov/ books/NBK321623/. [Last accessed on 2019 Jul 15].

563. Kokoska LA, Wilhelm SM, Garwood CL, Berlie HD. Aspirin for primary prevention of cardiovascular disease in patients with diabetes: A meta-analysis. Diabetes Res Clin Pract 2016;120:31-9.

564. Antithrombotic Trialists' (ATT) Collaboration, Baigent C, Blackwell L, Collins R, Emberson J, Godwin J, et al. Aspirin in the primary and secondary prevention of vascular disease: Collaborative meta-analysis of individual participant data from randomised trials. Lancet 2009;373:1849-60.

565. Tufano A, Cimino E, Di Minno MN, Ieranò P, Marrone E, Strazzullo A, et al. Diabetes mellitus and cardiovascular prevention: The role and the limitations of currently available antiplatelet drugs. Int J Vasc Med 2011;2011:250518.

566. Squizzato A, Keller T, Romualdi E, Middeldorp S. Clopidogrel plus aspirin versus aspirin alone for preventing cardiovascular disease. Cochrane Database Syst Rev 2011:CD005158.

567. Joshi P, Islam S, Pais P, Reddy S, Dorairaj P, Kazmi K, et al. Risk factors for early myocardial infarction in South Asians compared with individuals in other countries. JAMA 2007;297:286-94.

568. Knopp RH, d'Emden M, Smilde JG, Pocock SJ. Efficacy and safety of atorvastatin in the prevention of cardiovascular end points in subjects with type 2 diabetes: The atorvastatin study for prevention of coronary heart disease endpoints in non-insulin-dependent diabetes mellitus (ASPEN). Diabetes Care 2006;29:1478-85.

569. Macchia A, Laffaye N, Comignani PD, Cornejo Pucci E, Igarzabal C, Scazziota AS, et al. Statins but not aspirin reduce thrombotic risk assessed by thrombin generation in diabetic patients without cardiovascular events: The RATIONAL trial. PLoS One 2012;7:e32894.

570. Shepherd J, Barter P, Carmena R, Deedwania P, Fruchart JC, Haffner S, et al. Effect of lowering LDL cholesterol substantially below currently recommended levels in patients with coronary heart disease and diabetes: The Treating to New Targets (TNT) study. Diabetes Care 2006;29:1220-6.

571. de Vries FM, Kolthof J, Postma MJ, Denig P, Hak E. Efficacy of standard and intensive statin treatment for the secondary prevention of cardiovascular and cerebrovascular events in diabetes patients: A meta-analysis. PLoS One 2014;9:e111247.

572. Enas EA, Kuruvila A, Khanna P, Pitchumoni CS, Mohan V. Benefits risks of statin therapy for primary prevention of cardiovascular disease in Asian Indians - A population with the highest risk of premature coronary artery disease \& diabetes. Indian J Med Res 2013;138:461-91.

573. Gupta R, Lodha S, Sharma KK, Sharma SK, Gupta S, Asirvatham AJ, et al. Evaluation of statin prescriptions in type 2 diabetes: India heart watch-2. BMJ Open Diabetes Res Care 2016;4:e000275.

574. Doggrell SA. Is atorvastatin superior to other statins? Analysis of the clinical trials with atorvastatin having cardiovascular endpoints. Rev Recent Clin Trials 2006;1:143-53.

575. Cannon CP, Blazing MA, Giugliano RP, McCagg A, White JA, Theroux $\mathrm{P}$, et al. Ezetimibe added to statin therapy after acute coronary syndromes. N Engl J Med 2015;372:2387-97.

576. Foody JM, Toth PP, Tomassini JE, Sajjan S, Ramey DR, Neff D, et al. Changes in LDL-C levels and goal attainment associated with addition of ezetimibe to simvastatin, atorvastatin, or rosuvastatin compared with titrating statin monotherapy. Vasc Health Risk Manag 2013;9:719-27.

577. Keech A, Simes RJ, Barter P, Best J, Scott R, Taskinen MR, et al. Effects of long-term fenofibrate therapy on cardiovascular events in 9795 people with type 2 diabetes mellitus (the FIELD study): Randomised controlled trial. Lancet 2005;366:1849-61.

578. Goldfine AB, Kaul S, Hiatt WR. Fibrates in the treatment of dyslipidemias-time for a reassessment. N Engl J Med 2011;365:481-4.

579. Chaudhary R, Garg J, Shah N, Sumner A. PCSK9 inhibitors: A new era of lipid lowering therapy. World J Cardiol 2017;9:76-91.

580. Palmer SC, Mavridis D, Nicolucci A, Johnson DW, Tonelli M, Craig JC, et al. Comparison of clinical outcomes and adverse events associated with glucose-lowering drugs in patients with type 2 diabetes: A Meta-analysis. JAMA 2016;316:313-24.

581. American Diabetes Association Press Release. Linagliptin and Glimepiride Have Comparable Cardiovascular Safety Effects in Type 2 Diabetes at High Cardiovascular Risk; 2019. Available from: http:// www.diabetes.org/newsroom/press-releases/2019/linagliptin-andglimepiride.html. [Last accessed on $2019 \mathrm{Jul} 15]$.

582. Scirica BM, Braunwald E, Raz I, Cavender MA, Morrow DA, 
Jarolim $\mathrm{P}$, et al. Heart failure, saxagliptin, and diabetes mellitus: Observations from the SAVOR-TIMI 53 randomized trial. Circulation 2014;130:1579-88.

583. Karagiannis T, Bekiari E, Boura P, Tsapas A. Cardiovascular risk with DPP-4 inhibitors: Latest evidence and clinical implications. Ther Adv Drug Saf 2016;7:36-8.

584. Law MR, Morris JK, Wald NJ. Use of blood pressure lowering drugs in the prevention of cardiovascular disease: Meta-analysis of 147 randomised trials in the context of expectations from prospective epidemiological studies. BMJ 2009;338:b1665.

585. Bangalore S, Kumar S, Volodarskiy A, Messerli FH. Blood pressure targets in patients with coronary artery disease: Observations from traditional and Bayesian random effects meta-analysis of randomised trials. Heart 2013;99:601-13.

586. Thomopoulos C, Parati G, Zanchetti A. Effects of blood pressure lowering on outcome incidence in hypertension: 7. Effects of more vs. less intensive blood pressure lowering and different achieved blood pressure levels - updated overview and meta-analyses of randomized trials. J Hypertens 2016;34:613-22.

587. Joshi SR, Yeolekar ME, Tripathi KK, Giri J, Maity AK, Chopda M, et al. Evaluation of efficacy and tolerability of Losartan and Ramipril combination in the management of hypertensive patients with associated diabetes mellitus in India (LORD Trial). J Assoc Physicians India 2004;52:189-95.

588. Pareek A, Chandurkar NB, Sharma R, Tiwari D, Gupta BS. Efficacy and tolerability of a fixed-dose combination of metoprolol extended release/amlodipine in patients with mild-to-moderate hypertension: A randomized, parallel-group, multicentre comparison with losartan plus amlodipine. Clin Drug Investig 2010;30:123-31.

589. Rao NS, Oomman A, Bindumathi PL, Sharma V, Rao S, Moodahadu LS, et al. Efficacy and tolerability of fixed dose combination of metoprolol and amlodipine in Indian patients with essential hypertension. J Midlife Health 2013;4:160-6.

590. Cefalu WT, Kaul S, Gerstein HC, Holman RR, Zinman B, Skyler JS, et al. Cardiovascular outcomes trials in type 2 diabetes: Where do we go from here? Reflections from a diabetes care editors' expert forum. Diabetes Care 2018;41:14-31.

591. Testa R, Bonfigli AR, Prattichizzo F, La Sala L, De Nigris V, Ceriello A. The "metabolic memory" theory and the early treatment of hyperglycemia in prevention of diabetic complications. Nutrients 2017;9. pii: E437.

592. Gæde P, Oellgaard J, Carstensen B, Rossing P, Lund-Andersen H, Parving $\mathrm{HH}$, et al. Years of life gained by multifactorial intervention in patients with type 2 diabetes mellitus and microalbuminuria: 21 years follow-up on the Steno-2 randomised trial. Diabetologia 2016;59:2298-307.

593. Haslam DW, James WP. Obesity. Lancet 2005;366:1197-209.

594. Association American Diabetes. Updates to the standards of medical care in diabetes-2018. Diabetes Care 2018;41:2045-7.

595. World Health Organization. The Asia-Pacific Perspective: Redefining Obesity and its Treatment. Sydney: Health Communications Australia; 2000.

596. Ranjani H, Mehreen TS, Pradeepa R, Anjana RM, Garg R, Anand $\mathrm{K}$, et al. Epidemiology of childhood overweight obesity in India: A systematic review. Indian J Med Res 2016;143:160-74.

597. Pradeepa R, Anjana RM, Joshi SR, Bhansali A, Deepa M, Joshi PP, et al. Prevalence of generalized abdominal obesity in urban rural India-the ICMR-INDIAB Study (Phase-I) [ICMR-NDIAB-3]. Indian J Med Res 2015;142:139-50.

598. Misra A, Khurana L. Obesity and the metabolic syndrome in developing countries. J Clin Endocrinol Metab 2008;93:S9-30.

599. Ahirwar R, Mondal PR. Prevalence of obesity in India: A systematic review. Diabetes Metab Syndr 2019;13:318-21.

600. Misra A, Khurana L. The metabolic syndrome in South Asians: Epidemiology, determinants, and prevention. Metab Syndr Relat Disord 2009;7:497-514.

601. Misra A, Vikram NK, Gupta R, Pandey RM, Wasir JS, Gupta VP. Waist circumference cutoff points and action levels for Asian Indians for identification of abdominal obesity. Int J Obes (Lond) 2006;30:106-11.

602. Pandya H, Lakhani J, Patel N. Obesity is becoming synonym for diabetes in rural areas of india also an alarming situation. Int J Biol Med Res 2011;2:556-60.

603. Mohan V, Sharp PS, Cloke HR, Burrin JM, Schumer B, Kohner EM. Serum immunoreactive insulin responses to a glucose load in Asian Indian and European type 2 (non-insulin-dependent) diabetic patients and control subjects. Diabetologia 1986;29:235-7.

604. Snehalatha C, Viswanathan V, Ramachandran A. Cutoff values for normal anthropometric variables in Asian Indian adults. Diabetes Care $2003 ; 26: 1380-4$

605. Ramachandran A. Diabetes obesity - The Indian angle. Indian J Med Res 2004;120:437-9.

606. Kaur J. A comprehensive review on metabolic syndrome. Cardiol Res Pract 2014;2014:943162.

607. Chan JM, Rimm EB, Colditz GA, Stampfer MJ, Willett WC. Obesity, fat distribution, and weight gain as risk factors for clinical diabetes in men. Diabetes Care 1994;17:961-9.

608. Boschmann M, Michalsen A. Fasting therapy - old and new perspectives. Forsch Komplementmed 2013;20:410-1.

609. Hassanein M, Al-Arouj M, Hamdy O, Bebakar WM, Jabbar A, Al-Madani A, et al. Diabetes and ramadan: Practical guidelines. Diabetes Res Clin Pract 2017;126:303-16.

610. Misra A, Chowbey P, Makkar BM, Vikram NK, Wasir JS, Chadha D, et al. Consensus statement for diagnosis of obesity, abdominal obesity and the metabolic syndrome for Asian Indians and recommendations for physical activity, medical and surgical management. J Assoc Physicians India 2009;57:163-70.

611. Misra A. Revisions of cutoffs of body mass index to define overweight and obesity are needed for the Asian-ethnic groups. Int J Obes Relat Metab Disord 2003;27:1294-6.

612. Misra A. Body composition and the metabolic syndrome in Asian Indians: A saga of multiple adversities. Natl Med J India 2003;16:3-7.

613. McKeigue PM, Shah B, Marmot MG. Relation of central obesity and insulin resistance with high diabetes prevalence and cardiovascular risk in South Asians. Lancet 1991;337:382-6.

614. Misra A. We need ethnic-specific criteria for classification of BMI. Prog Obes Res 2003;9:547-53.

615. Fujimoto WY, Jablonski KA, Bray GA, Kriska A, Barrett-Connor E, Haffner S, et al. Body size and shape changes and the risk of diabetes in the diabetes prevention program. Diabetes 2007;56:1680-5.

616. Look AHEAD Research Group, Wing RR. Long-term effects of a lifestyle intervention on weight and cardiovascular risk factors in individuals with type 2 diabetes mellitus: Four-year results of the Look AHEAD trial. Arch Intern Med 2010;170:1566-75.

617. Gurka MJ, Wolf AM, Conaway MR, Crowther JQ, Nadler JL, Bovbjerg VE. Lifestyle intervention in obese patients with type 2 diabetes: Impact of the patient's educational background. Obesity (Silver Spring) 2006;14:1085-92.

618. Misra A, Sharma R, Gulati S, Joshi SR, Sharma V, Ghafoorunissa, et al. Consensus dietary guidelines for healthy living and prevention of obesity, the metabolic syndrome, diabetes, and related disorders in Asian Indians. Diabetes Technol Ther 2011;13:683-94.

619. Sahu RK, Prashar D. Current treatment strategies for obesity including Indian scenario. Asian J Pharm 2016;10:S342-9.

620. Pascale RW, Wing RR, Butler BA, Mullen M, Bononi P. Effects of a behavioral weight loss program stressing calorie restriction versus calorie plus fat restriction in obese individuals with NIDDM or a family history of diabetes. Diabetes Care 1995;18:1241-8.

621. Avenell A, Broom J, Brown TJ, Poobalan A, Aucott L, Stearns SC, et al. Systematic review of the long-term effects and economic consequences of treatments for obesity and implications for health improvement. Health Technol Assess 2004;8:iii-iv, 1-182.

622. Brown T, Smith S, Bhopal R, Kasim A, Summerbell C. Diet and physical activity interventions to prevent or treat obesity in South Asian children and adults: A systematic review and meta-analysis. Int J Environ Res Public Health 2015;12:566-94.

623. Swift DL, Johannsen NM, Lavie CJ, Earnest CP, Church TS. The role of exercise and physical activity in weight loss and maintenance. Prog Cardiovasc Dis 2014;56:441-7.

624. Williamson DF, Madans J, Anda RF, Kleinman JC, Kahn HS, Byers T. Recreational physical activity and ten-year weight change in a US 
national cohort. Int J Obes Relat Metab Disord 1993;17:279-86.

625. Littman AJ, Kristal AR, White E. Effects of physical activity intensity, frequency, and activity type on 10-y weight change in middle-aged men and women. Int J Obes (Lond) 2005;29:524-33

626. Ballor DL, Smith DB, Tommerup LJ, Thomas DP. Neither high- nor low-intensity exercise promotes whole-body conservation of protein during severe dietary restrictions. Int J Obes 1990;14:279-87.

627. Melanson EL, MacLean PS, Hill JO. Exercise improves fat metabolism in muscle but does not increase 24-h fat oxidation. Exerc Sport Sci Rev 2009;37:93-101.

628. Church TS, Blair SN, Cocreham S, Johannsen N, Johnson W, Kramer K, et al. Effects of aerobic and resistance training on hemoglobin A1c levels in patients with type 2 diabetes: A randomized controlled trial. JAMA 2010;304:2253-62.

629. World Health Organization. Global Strategy on Diet, Physical Activity and Health Global Strategy on Diet. Physical Activity and Health; 2010.

630. Lean ME, Leslie WS, Barnes AC, Brosnahan N, Thom G, McCombie L, et al. Primary care-led weight management for remission of type 2 diabetes (DiRECT): An open-label, cluster-randomised trial. Lancet 2018;391:541-51

631. Lean MEJ, Leslie WS, Barnes AC, Brosnahan N, Thom G, McCombie L, et al. Durability of a primary care-led weight-management intervention for remission of type 2 diabetes: 2-year results of the DiRECT open-label, cluster-randomised trial. Lancet Diabetes Endocrinol 2019;7:344-55

632. Fogelholm M, Larsen TM, Westerterp-Plantenga M, Macdonald I, Martinez JA, Boyadjieva N, et al. PREVIEW: Prevention of diabetes through lifestyle intervention and population studies in europe and around the world. Design, methods, and baseline participant description of an adult cohort enrolled into a three-year randomised clinical trial. Nutrients 2017;9. pii: E632.

633. Swindell N, Mackintosh K, McNarry M, Stephens JW, Sluik D, Fogelholm M, et al. Objectively measured physical activity and sedentary time are associated with cardiometabolic risk factors in adults with prediabetes: The PREVIEW study. Diabetes Care 2018;41:562-9.

634. Christensen P, Meinert Larsen T, Westerterp-Plantenga M, Macdonald I, Martinez JA, Handjiev S, et al. Men and women respond differently to rapid weight loss: Metabolic outcomes of a multi-centre intervention study after a low-energy diet in 2500 overweight, individuals with pre-diabetes (PREVIEW). Diabetes Obes Metab 2018;20:2840-51.

635. Hendricks EJ. Off-label drugs for weight management. Diabetes Metab Syndr Obes 2017;10:223-34

636. Lee A, Morley JE. Metformin decreases food consumption and induces weight loss in subjects with obesity with type II non-insulin-dependent diabetes. Obes Res 1998;6:47-53.

637. Diabetes Prevention Program Research Group. Long-term safety, tolerability, and weight loss associated with metformin in the diabetes prevention program outcomes study. Diabetes Care 2012;35:731-7.

638. Gupta S, Shaikh S, Joshi P, Bhure S, Suvarna V. Long-term efficacy and safety of empagliflozin monotherapy in drug-naïve patients with type 2 diabetes in Indian subgroup: Results from a 76-week extension trial of phase III, double-blind, randomized study. Indian J Endocrinol Metab 2017;21:286-92

639. Saboo B, Reddy GC, Juneja S, Kedia AK, Manjrekar P, Rathod R, et al. Effectiveness and safety of fixed dose combination of acarbose/ metformin in Indian Type 2 diabetes patients: Results from observational GLOBE Study. Indian J Endocrinol Metab 2015;19:129-35.

640. Aldekhail NM, Logue J, McLoone P, Morrison DS. Effect of orlistat on glycaemic control in overweight and obese patients with type 2 diabetes mellitus: A systematic review and meta-analysis of randomized controlled trials. Obes Rev 2015;16:1071-80.

641. O'Neil PM, Smith SR, Weissman NJ, Fidler MC, Sanchez M, Zhang J, et al. Randomized placebo-controlled clinical trial of lorcaserin for weight loss in type 2 diabetes mellitus: The BLOOM-DM study. Obesity (Silver Spring) 2012;20:1426-36.

642. Bohula EA, Scirica BM, Inzucchi SE, McGuire DK, Keech AC, Smith SR, et al. Effect of lorcaserin on prevention and remission of type 2 diabetes in overweight and obese patients (CAMELLIA-TIMI 61): A randomised, placebo-controlled trial. Lancet 2018;392:2269-79.
643. Bohula EA, Wiviott SD, McGuire DK, Inzucchi SE, Kuder J, Im K, et al. Cardiovascular safety of lorcaserin in overweight or obese patients. N Engl J Med 2018;379:1107-17.

644. FDA Requests the Withdrawal of the Weight-Loss Drug Belviq, Belviq XR (Lorcaserin) from the market USA: U. S. Food and Drug Administration; 2020. Available from: https://www.fda.gov/ media/135189/download. [Last accessed on 2020 Feb].

645. Kashyap SR, Bhatt DL, Wolski K, Watanabe RM, Abdul-Ghani M, Abood B, et al. Metabolic effects of bariatric surgery in patients with moderate obesity and type 2 diabetes: Analysis of a randomized control trial comparing surgery with intensive medical treatment. Diabetes Care 2013;36:2175-82

646. Schauer PR, Bhatt DL, Kirwan JP, Wolski K, Aminian A, Brethauer SA, et al. Bariatric surgery versus intensive medical therapy for diabetes - 5-year outcomes. N Engl J Med 2017;376:641-51.

647. Schauer PR, Bhatt DL, Kirwan JP, Wolski K, Brethauer SA, Navaneethan SD, et al. Bariatric surgery versus intensive medical therapy for diabetes-3-year outcomes. N Engl J Med 2014;370:2002-13.

648. Schauer PR, Kashyap SR, Wolski K, Brethauer SA, Kirwan JP, Pothier CE, et al. Bariatric surgery versus intensive medical therapy in obese patients with diabetes. N Engl J Med 2012;366:1567-76.

649. Yan Y, Sha Y, Yao G, Wang S, Kong F, Liu H, et al. Roux-en-Y gastric bypass versus medical treatment for type 2 diabetes mellitus in obese patients: A systematic review and meta-analysis of randomized controlled trials. Medicine (Baltimore) 2016;95:e3462.

650. Singhai A, Sharma P, Jha RK. Effect of sleeve gastrectomy and gastric bypass on diabetic control in Indore, India. Saudi J Obes 2014;2:59.

651. Bhasker AG, Remedios C, Batra P, Sood A, Shaikh S, Lakdawala M. Predictors of remission of T2DM and metabolic effects after laparoscopic roux-en-y gastric bypass in obese indian diabetics-a 5-year study. Obes Surg 2015;25:1191-7.

652. Dasgupta A, Wasir J, Beloyartseva M, Malhotra S, Mithal A. An observational longitudinal study of the impact of sleeve gastrectomy on glycemic control in type 2 diabetes mellitus. Diabetes Technol Ther 2013;15:990-5.

653. Lakdawala M, Shaikh S, Bandukwala S, Remedios C, Shah M, Bhasker AG. Roux-en-Y gastric bypass stands the test of time: 5-year results in low body mass index (30-35 $\mathrm{kg} / \mathrm{m}(2))$ Indian patients with type 2 diabetes mellitus. Surg Obes Relat Dis 2013;9:370-8.

654. Weight-Loss and Weight-Management Devices; 2019. Available from: https://www.fda.gov/medical-devices/products-and-medicalprocedures/weight-loss-and-weight-management-devices. [Last accessed on 2019 Aug 20].

655. Sullivan S. Endoscopic medical devices for primary obesity treatment in patients with diabetes. Diabetes Spectr 2017;30:258-64.

656. Gallagher EJ, LeRoith D. Obesity and diabetes: The increased risk of cancer and cancer-related mortality. Physiol Rev 2015;95:727-48.

657. Belfiore A, Frasca F, Pandini G, Sciacca L, Vigneri R. Insulin receptor isoforms and insulin receptor/insulin-like growth factor receptor hybrids in physiology and disease. Endocr Rev 2009;30:586-623.

658. de Beer JC, Liebenberg L. Does cancer risk increase with HbA1c, independent of diabetes? Br J Cancer 2014;110:2361-8.

659. Melvin JC, Holmberg L, Rohrmann S, Loda M, van Hemelrijck M. Serum lipid profiles and cancer risk in the context of obesity: Four meta-analyses. J Cancer Epidemiol 2013;2013:823849.

660. Maiorino MI, Bellastella G, Esposito K. Diabetes and sexual dysfunction: Current perspectives. Diabetes Metab Syndr Obes 2014;7:95-105.

661. Enzlin P, Rosen R, Wiegel M, Brown J, Wessells H, Gatcomb P, et al. Sexual dysfunction in women with type 1 diabetes: Long-term findings from the DCCT/ EDIC study cohort. Diabetes Care 2009;32:780-5.

662. Feldman HA, Goldstein I, Hatzichristou DG, Krane RJ, McKinlay JB. Impotence and its medical and psychosocial correlates: Results of the Massachusetts Male Aging Study. J Urol 1994;151:54-61.

663. Kouidrat Y, Pizzol D, Cosco T, Thompson T, Carnaghi M, Bertoldo A, et al. High prevalence of erectile dysfunction in diabetes: A systematic review and meta-analysis of 145 studies. Diabet Med 2017;34:1185-92.

664. Sondhi M, Kakar A, Gogia A, et al. Prevalence of erectile dysfunction in diabetic patients. Curr Med Res Pract 2018;8:88-91.

665. Johannes CB, Araujo AB, Feldman HA, Derby CA, Kleinman KP, 
McKinlay JB. Incidence of erectile dysfunction in men 40 to 69 years old: Longitudinal results from the Massachusetts male aging study. J Urol 2000;163:460-3.

666. Angulo J, Cuevas $\mathrm{P}$, Fernández A, Gabancho S, Allona A, Martín-Morales A, et al. Diabetes impairs endothelium-dependent relaxation of human penile vascular tissues mediated by NO and EDHF. Biochem Biophys Res Commun 2003;312:1202-8.

667. Kapoor D, Clarke S, Channer KS, Jones TH. Erectile dysfunction is associated with low bioactive testosterone levels and visceral adiposity in men with type 2 diabetes. Int J Androl 2007;30:500-7.

668. American Diabetes Association. 6. Glycemic targets: Standards of medical care in diabetes-2019. Diabetes Care 2019;42:S61-S70.

669. Bajaj S, Jawad F, Islam N, Mahtab H, Bhattarai J, Shrestha D, et al. South Asian women with diabetes: Psychosocial challenges and management: Consensus statement. Indian J Endocrinol Metab 2013;17:548-62.

670. Pontiroli AE, Cortelazzi D, Morabito A. Female sexual dysfunction and diabetes: A systematic review and meta-analysis. J Sex Med 2013; 10:1044-51.

671. Basson R, Berman J, Burnett A, Derogatis L, Ferguson D, Fourcroy J, et al. Report of the international consensus development conference on female sexual dysfunction: Definitions and classifications. J Urol 2000;163:888-93.

672. Kalra B, SK, Chawla K, Batra P, Chhabra B. Sexual attitudes, knowledge and function of women with diabetes. Int J Geriatr Gerontol 2009;5:2.

673. Seftel AD, Sun P, Swindle R. The prevalence of hypertension, hyperlipidemia, diabetes mellitus and depression in men with erectile dysfunction. J Urol 2004;171:2341-5.

674. Rosen RC. Sexual dysfunction as an obstacle to compliance with antihypertensive therapy. Blood Press Suppl 1997;1:47-51.

675. Yamada T, Hara K, Umematsu H, Suzuki R, Kadowaki T. Erectile dysfunction and cardiovascular events in diabetic men: A meta-analysis of observational studies. PLoS One 2012;7:e43673.

676. Meena BL, Kochar DK, Agarwal TD, Choudhary R, Kochar A. Association between erectile dysfunction and cardiovascular risk in individuals with type-2 diabetes without overt cardiovascular disease. Int J Diabetes Dev Ctries 2009;29:150-4.

677. Alexopoulou O, Jamart J, Maiter D, Hermans MP, de Hertogh R, de Nayer $\mathrm{P}$, et al. Erectile dysfunction and lower androgenicity in type 1 diabetic patients. Diabetes Metab 2001;27:329-36.

678. Kalinchenko SY, Kozlov GI, Gontcharov NP, Katsiya GV. Oral testosterone undecanoate reverses erectile dysfunction associated with diabetes mellitus in patients failing on sildenafil citrate therapy alone. Aging Male 2003;6:94-9

679. Agarwal PK, Singh P, Chowdhury S, Sharma SK, Majumdar A, Shah P, et al. A study to evaluate the prevalence of hypogonadism in Indian males with Type-2 diabetes mellitus. Indian J Endocrinol Metab 2017;21:64-70.

680. Wing RR, Rosen RC, Fava JL, Bahnson J, Brancati F, Gendrano Iii IN, et al. Effects of weight loss intervention on erectile function in older men with type 2 diabetes in the Look AHEAD trial. $\mathrm{J}$ Sex Med 2010;7:156-65.

681. Anwar Z, Sinha V, Mitra S, Mishra AK, Ansari MH, Bharti A, et al. Erectile Dysfunction: An Underestimated Presentation in Patients with Diabetes Mellitus. Indian J Psychol Med 2017;39:600-4.

682. Esposito K, Maiorino MI, Bellastella G, Giugliano F, Romano M, Giugliano D. Determinants of female sexual dysfunction in type 2 diabetes. Int J Impot Res 2010;22:179-84.

683. LeRoith D, Smith DO. Monitoring glycemic control: The cornerstone of diabetes care. Clin Ther 2005;27:1489-99.

684. Rao PV, Makkar BM, Kumar A, et al. RSSDI consensus on self-monitoring of blood glucose in types 1 and 2 diabetes mellitus in India. Int J Diabetes Dev Ctries 2018;38:260-79.

685. Pimazoni-Netto A, Rodbard D, Zanella MT, Diabetes Education and Control Group. Rapid improvement of glycemic control in type 2 diabetes using weekly intensive multifactorial interventions: Structured glucose monitoring, patient education, and adjustment of therapy-a randomized controlled trial. Diabetes Technol Ther 2011;13:997-1004.
686. Rahaghi FN, Gough DA. Blood glucose dynamics. Diabetes Technol Ther 2008;10:81-94.

687. Kovatchev BP. Diabetes technology: Markers, monitoring, assessment, and control of blood glucose fluctuations in diabetes. Scientifica (Cairo) 2012;2012:283821.

688. WHO Guidelines Approved by the Guidelines Review Committee. Use of Glycated Haemoglobin (HbAlc) in the Diagnosis of Diabetes Mellitus: Abbreviated Report of a WHO Consultation. WHO Guidelines Approved by the Guidelines Review Committee. Geneva: World Health Organization; 2011.

689. Golden S, Boulware LE, Berkenblit G, Brancati F, Chander G, Marinopoulos S, et al. Use of glycated hemoglobin and microalbuminuria in the monitoring of diabetes mellitus. Evid Rep Technol Assess (Summ) 2003:1-6.

690. Gangopadhyay KK, Singh P. Consensus statement on dose modifications of antidiabetic agents in patients with hepatic impairment. Indian J Endocrinol Metab 2017;21:341-54.

691. Rahman S, Rahman T, Ismail AA, Rashid AR. Diabetes-associated macrovasculopathy: Pathophysiology and pathogenesis. Diabetes Obes Metab 2007;9:767-80.

692. Kirk JK, Stegner J. Self-monitoring of blood glucose: Practical aspects. J Diabetes Sci Technol 2010;4:435-9.

693. Bailey TS, Grunberger G, Bode BW, Handelsman Y, Hirsch IB, Jovanovič L, et al. American association of clinical endocrinologists and American college of endocrinology 2016 outpatient glucose monitoring consensus statement. Endocr Pract 2016;22:231-61.

694. Klonoff DC, Buckingham B, Christiansen JS, Montori VM, Tamborlane WV, Vigersky RA, et al. Continuous glucose monitoring: An Endocrine Society Clinical Practice Guideline. J Clin Endocrinol Metab 2011;96:2968-79.

695. Healy SJ, Dungan KM. Monitoring glycemia in diabetes. Med Clin North Am 2015;99:35-45.

696. Saboo B, Chawla M, Jha S, et al. Consensus and recommendations on continuous glucose monitoring. J Diabetol 2019;10:4.

697. Khadilkar KS, Bandgar T, Shivane V, Lila A, Shah N. Current concepts in blood glucose monitoring. Indian J Endocrinol Metab 2013;17:S643-9.

698. Rao P, Makkar B, Kumar A, et al. RSSDI consensus on self-monitoring of blood glucose in types 1 and 2 diabetes mellitus in India. Int J Diabetes Dev Ctries 2018;38:260-79.

699. Schnell O, Hanefeld M, Monnier L. Self-monitoring of blood glucose: A prerequisite for diabetes management in outcome trials. J Diabetes Sci Technol 2014;8:609-14.

700. Mansouri DA, Alawi HH, Barasyn KB, et al. Self-monitoring of blood glucose among diabetic patients attending Al-Eskan Primary Health Care Center in Makkah Al-Mukarramah city. Int J Med Sci Public Health 2015;4:527-38.

701. Karter AJ, Ferrara A, Darbinian JA, Ackerson LM, Selby JV. Self-monitoring of blood glucose: Language and financial barriers in a managed care population with diabetes. Diabetes Care 2000;23:477-83.

702. Shrivastava SR, Shrivastava PS, Ramasamy J. Role of self-care in management of diabetes mellitus. J Diabetes Metab Disord 2013;12:14.

703. Recommendations for Managing Type 2 Diabetes in Primary Care; 2017. Available from: www.idf.org/managing-type2-diabetes. [Last accessed on $2019 \mathrm{Jul}]$.

704. Chowdhury S, Ji L, Suwanwalaikorn S, Yu NC, Tan EK. Practical approaches for self-monitoring of blood glucose: An Asia-Pacific perspective. Curr Med Res Opin 2015;31:461-76.

705. Unnikrishnan R, Mohan V. Suggested Protocols for Self-Monitoring of Blood Glucose in India. The Association of Physicians of India; 2013. p. 194-7.

706. American Diabetes Association. 12. Older adults: Standards of medical care in diabetes-2019. Diabetes Care 2019;42:S139-47.

707. American Geriatrics Society Expert Panel on Care of Older Adults with Diabetes Mellitus, Moreno G, Mangione CM, Kimbro L, Vaisberg E. Guidelines abstracted from the American geriatrics society guidelines for improving the care of older adults with diabetes mellitus: 2013 update. J Am Geriatr Soc 2013;61:2020-6.

708. Larsen ML, Hørder M, Mogensen EF. Effect of long-term monitoring of glycosylated hemoglobin levels in insulin-dependent diabetes 
mellitus. N Engl J Med 1990;323:1021-5.

709. Sherwani SI, Khan HA, Ekhzaimy A, Masood A, Sakharkar MK. Significance of HbAlc test in diagnosis and prognosis of diabetic patients. Biomark Insights 2016;11:95-104.

710. Heisler M, Piette JD, Spencer M, Kieffer E, Vijan S. The relationship between knowledge of recent $\mathrm{HbAlc}$ values and diabetes care understanding and self-management. Diabetes Care 2005;28:816-22.

711. Nathan DM, Kuenen J, Borg R, Zheng H, Schoenfeld D, Heine RJ, et al. Translating the $\mathrm{A} 1 \mathrm{C}$ assay into estimated average glucose values. Diabetes Care 2008;31:1473-8.

712. Betônico CC, Titan SM, Correa-Giannella ML, Nery M, Queiroz M. Management of diabetes mellitus in individuals with chronic kidney disease: Therapeutic perspectives and glycemic control. Clinics (Sao Paulo) 2016;71:47-53.

713. Sacks DB. Hemoglobin variants and hemoglobin A1c analysis: Problem solved? Clin Chem 2003;49:1245-7.

714. Rajagopal L, Arunachalam S, Ganapathy S, et al. Impact of iron deficiency anemia on glycated hemoglobin (HbA1c) levels in diabetics with controlled plasma glucose levels. Ann Pathol Lab Med 2017;4:A148-52.

715. Mohan V, Deepa R, Shefali AK, Poongothai S, Monica M, Karkuzhali K. Evaluation of One Touch HORIZON-a highly affordable glucose monitor. J Assoc Physicians India 2004;52:779-82.

716. Shaji S, Rajendran D, Kumpatla S, et al. Evaluation of diabetes self-care with self-monitoring of blood glucose among type 2 diabetic patients and its impact on HbA1c. Int J Diabetes Dev Ctries 2013;33:181.

717. Silva DD, Bosco AA. An educational program for insulin self-adjustment associated with structured self-monitoring of blood glucose significantly improves glycemic control in patients with type 2 diabetes mellitus after 12 weeks: A randomized, controlled pilot study. Diabetol Metab Syndr 2015;7:2.

718. Karter AJ, Ackerson LM, Darbinian JA, D'Agostino RB Jr., Ferrara A, Liu J, et al. Self-monitoring of blood glucose levels and glycemic control: The Northern California Kaiser Permanente Diabetes registry. Am J Med 2001;111:1-9.

719. Kibriya MG, Ali L, Banik NG, Khan AK. Home monitoring of blood glucose (HMBG) in Type-2 diabetes mellitus in a developing country. Diabetes Res Clin Pract 1999;46:253-7.

720. Mast O, Tan A, Punjabi K. Usage of Self-Monitoring of Blood Glucose (Smbg) By Diabetes Therapy Type in India. Value Health 2014;17:A362.

721. Garg S, Zisser H, Schwartz S, Bailey T, Kaplan R, Ellis S, et al. Improvement in glycemic excursions with a transcutaneous, real-time continuous glucose sensor: A randomized controlled trial. Diabetes Care 2006;29:44-50.

722. Mohan V, Jain S, Kesavadev J, Chawla M, Mutha A, Viswanathan V, et al. Use of retrospective continuous glucose monitoring for optimizing management of type 2 diabetes in India. J Assoc Physicians India 2016;64:16-21.

723. American Diabetes Association. Introduction: Standards of Medical Care in Diabetes-2019. Diabetes Care 2019;42 Suppl 1:S1-2.

724. Grunberger G, Handelsman Y, Bloomgarden ZT, Fonseca VA, Garber AJ, Haas RA, et al. American association of clinical endocrinologists and American college of endocrinology 2018 position statement on integration of insulin pumps and continuous glucose monitoring in patients with diabetes mellitus. Endocr Pract 2018;24:302-8.

725. Grunberger G, Abelseth JM, Bailey TS, Bode BW, Handelsman Y, Hellman R, et al. Consensus Statement by the American Association of Clinical Endocrinologists/American College of Endocrinology insulin pump management task force. Endocr Pract 2014;20:463-89.

726. Kesavadev J, Sadikot S, Wangnoo S, Kannampilly J, Saboo B, Aravind SR, et al. Consensus guidelines for glycemic monitoring in type 1/type 2 \& GDM. Diabetes Metab Syndr 2014;8:187-95.

727. Kanungo A, Jhingan A, Sahay RK, Muruganathan A, Das AK; Diabetes Consensus Group. Consensus evidence-based guidelines for insulin therapy in patients with type 1 diabetes mellitus as per Indian clinical practice. J Assoc Physicians India 2014;62:26-33.

728. Kesavadev J, Jain SM, Muruganathan A, Das AK; Diabetes Consensus Group. Consensus evidence-based guidelines for use of insulin pump therapy in the management of diabetes as per Indian clinical practice. $\mathrm{J}$ Assoc Physicians India 2014;62:34-41.

729. Seshiah V, Banerjee S, Balaji V, Muruganathan A, Das AK; Diabetes Consensus Group. Consensus evidence-based guidelines for management of gestational diabetes mellitus in India. J Assoc Physicians India 2014;62:55-62.

730. Peters AL, Ahmann AJ, Battelino T, Evert A, Hirsch IB, Murad MH, et al. Diabetes technology-continuous subcutaneous insulin infusion therapy and continuous glucose monitoring in adults: An Endocrine Society clinical practice guideline. J Clin Endocrinol Metab 2016;101:3922-37.

731. Sherr JL, Tauschmann M, Battelino T, de Bock M, Forlenza G, Roman R, et al. ISPAD clinical practice consensus guidelines 2018: Diabetes technologies. Pediatr Diabetes 2018;19 Suppl 27:302-25.

732. Kesavadev J, Das AK, Unnikrishnan R 1 $1^{\text {st }}$, Joshi SR, Ramachandran A, Shamsudeen J, et al. Use of insulin pumps in India: Suggested guidelines based on experience and cultural differences. Diabetes Technol Ther 2010;12:823-31.

733. Bergenstal RM, Gavin JR $3^{\text {rd }}$; Global Consensus Conference on Glucose Monitoring Panel. The role of self-monitoring of blood glucose in the care of people with diabetes: Report of a global consensus conference. Am J Med 2005;118:1S-6S.

734. Clarke SF, Foster JR. A history of blood glucose meters and their role in self-monitoring of diabetes mellitus. Br J Biomed Sci 2012;69:83-93.

735. Nathan DM; DCCT/EDIC Research Group. The diabetes control and complications trial/epidemiology of diabetes interventions and complications study at 30 years: Overview. Diabetes Care 2014;37:9-16.

736. Kato N, Cui J, Kato M. Structured self-monitoring of blood glucose reduces glycated hemoglobin in insulin-treated diabetes. J Diabetes Investig 2013;4:450-3.

737. Kempf K, Kruse J, Martin S. ROSSO-in-praxi follow-up: Long-term effects of self-monitoring of blood glucose on weight, hemoglobin A1c, and quality of life in patients with type 2 diabetes mellitus. Diabetes Technol Ther 2012;14:59-64.

738. Miller KM, Beck RW, Bergenstal RM, Goland RS, Haller MJ, McGill JB, et al. Evidence of a strong association between frequency of self-monitoring of blood glucose and hemoglobin A1c levels in T1D exchange clinic registry participants. Diabetes Care 2013;36:2009-14.

739. U.S. Food and Drug Administration. Blood Glucose Monitoring Devices; 2018. Available from: https://www.fda.gov/ medicaldevices/productsandmedicalprocedures/invitrodiagnostics/ glucosetestingdevices/default.htm. [Last updated on 2018 Sep 27, Last accessed on 2019 Feb 21].

740. International Organization for Standardization. ISO 15197: 2013 In vitro Diagnostic Test Systems-Requirements for Blood-Glucose Monitoring Systems for self-Testing in Managing Diabetes Mellitus. [Last accessed on 2019 Mar 04].

741. U.S. Food and Drug Adminstration. Self-Monitoring Blood Glucose Test Systems for Over-the-Counter Use. Guidance for Industry and Food and Drug Administration Staff; 2016. Available from: https://www. fda.gov/downloads/MedicalDevices/DeviceRegulationandGuidance/ GuidanceDocuments/UCM380327.pdf. [Last accessed on 2019 Mar 04].

742. U.S. Food and Drug Administration. Blood Glucose Monitoring Test Systems for Prescription Point-of-Care Use. Guidance for Industry and Food and Drug Administration Staff; 2016. Available from: https://www.fda.gov/downloads/MedicalDevices/ DeviceRegulationandGuidance/GuidanceDocuments/UCM380325. pdf. [Last accessed on 2019 Mar 04].

743. American Diabetes Association. 7. Diabetes technology: Standards of medical care in diabetes-2019. Diabetes Care 2019:42:S71-80.

744. Kesavadev J, Ramachandran L, Krishnan G. Glucose monitoring technologies-complementary or competitive? Role of continuous glucose monitoring versus flash glucose monitoring versus self-monitoring of blood glucose. J Diabetol 2017;8:61.

745. Hirsch IB, Brownlee M. Should minimal blood glucose variability become the gold standard of glycemic control? J Diabetes Complications 2005;19:178-81.

746. Bolli GB. Hypoglycaemia unawareness. Diabetes Metab 
1997;23 Suppl 3:29-35

747. Gold AE, MacLeod KM, Frier BM. Frequency of severe hypoglycemia in patients with type I diabetes with impaired awareness of hypoglycemia. Diabetes Care 1994;17:697-703.

748. Kesavadev J, Ramachandran L, Krishnan G. Glucose monitoring technologies - complementary or competitive? Role of continuous glucose monitoring versus flash glucose monitoring versus self-monitoring of blood glucose. J Diabetol 2017;8:61-93.

749. Danne T, Nimri R, Battelino T, Bergenstal RM, Close KL, Devries JH, et al. International Consensus on Use of Continuous Glucose Monitoring. Diabetes Care 2017;40:1631-40.

750. Kesavadev J, Ramachandran L, Krishnan G. Glucose monitoring technologies - complementary or competitive? Role of continuous glucose monitoring versus flash glucose monitoring versus self-monitoring of blood glucose J Diabetol 2017;8:61-7.

751. Zisser H, Lane JE, Shivers JP. Continuous Glucose Monitoring: Professional and Real Time. Technological Advances in the Treatment of Type 1 Diabetes. Vol. 24. Karger Publishers; 2015. p. 81-98.

752. Rodbard D. Continuous glucose monitoring: A review of recent studies demonstrating improved glycemic outcomes. Diabetes Technol Ther 2017;19:S25-37.

753. Mamindla S, Koganti VS, Ravouru N, Koganti B. Effect of cinnamomum cassia on the pharmacokinetics and pharmacodynamics of pioglitazone. Curr Clin Pharmacol 2017;12:41-9.

754. Abbott. Real-World Data from Abbott's FreeStyle ${ }^{\circledR}$ Libre Show Association between Higher Frequency of Glucose Monitoring and Improved Glucose Control for People with Diabetes: Cision PR Newswire; 2017. Available from: https://www.prnewswire.com/ news-releases/real-world-data-from-abbotts-freestyle-libre-showassociation-between-higher-frequency-of-glucose-monitoring-andimproved-glucose-control-for-people-with-diabetes-300407487.html. [Last accessed on 2017 Dec 27].

755. Kesavadev J, Saboo B, Sadikot S, Das AK, Joshi S, Chawla R, et al. Unproven therapies for diabetes and their implications. Adv Ther 2017;34:60-77.

756. Medtronic MiniMed I. The Guardian ${ }^{\mathrm{TM}}$ Connect CGM. Available from: https://www.medtronicdiabetes.com/products/guardianconnect-continuous-glucose-monitoring-system. [Last accessed 2018 Aug 14].

757. Medtronic MiniMed I. iPro ${ }^{\mathrm{TM}} 2$ Professional Continuous Glucose Monitoring (CGM); 2017. http://professional.medtronicdiabetes.com/ ipro2-professional-cgm. [Last accessed on 2017 Dec 27].

758. Anhalt H, Bohannon NJ. Insulin patch pumps: Their development and future in closed-loop systems. Diabetes Technol Ther 2010;12 Suppl 1:S51-8.

759. Ginsberg BH. Patch pumps for insulin. J Diabetes Sci Technol 2019;13:27-33

760. Kesavadev J, Shankar A, David Ashok A. Free Insulin Pumps to Type 1 Diabetes Children- KT1DP Initiative. Abu Dhabi: International Diabetes Federation 2017 Congress; 2017.

761. Shah VN, Shoskes A, Tawfik B. Closed-Loop System in the Management of Diabetes: Past, Present, and Future. 140 Huguenot Street, $3^{\text {rd }}$ Floor New Rochelle, NY 10801 USA: Mary Ann Liebert, Inc.; 2014.

762. McAdams BH, Rizvi AA. An overview of insulin pumps and glucose sensors for the generalist. J Clin Med 2016;5. pii: E5.

763. Haidar A. The artificial pancreas: How closed-loop control is revolutionizing diabetes. IEEE Control Syst Mag 2016;36:28-47.

764. Medtronic MiniMed I. MiniMed ${ }^{\mathrm{TM}}$ 670G Insulin Pump System; 2018. Available from: https://www.medtronicdiabetes.com/ products/minimed-670g-insulin-pump-system. [Last accessed on 2018 August 14].

765. Healthline Media. Roche Solo Patch Insulin Pump Finally on the Way? Available from: https://www.healthline.com/diabetesmine/roche-solopatch-insulin-pump-coming\#1. [Last accessed on 2019 Mar 06].

766. Hoffmann-La Roche Ltd. Roche receives CE Mark for its Accu-Chek Solo Micropump System. Availablef from: https://www.roche. com/media/releases/med-cor-2018-07-23.htm. [Last accessed on 2019 Mar 06].

767. Corporation I. Omnipod System. Available from: https://www.
myomnipod.com/explore-Omnipod/Omnipod-system/. [Last accessed on 2017 Jul 10].

768. Thabit H, Hovorka R. Continuous subcutaneous insulin infusion therapy and multiple daily insulin injections in type 1 diabetes mellitus: A comparative overview and future horizons. Expert Opin Drug Deliv 2016;13:389-400.

769. Pickup JC, Reznik Y, Sutton AJ. Glycemic control during continuous subcutaneous insulin infusion versus multiple daily insulin injections in type 2 diabetes: Individual patient data meta-analysis and meta-regression of randomized controlled trials. Diabetes Care 2017;40:715-22.

770. Reznik Y, Cohen O, Aronson R, Conget I, Runzis S, Castaneda J, et al. Insulin pump treatment compared with multiple daily injections for treatment of type 2 diabetes (OpT2mise): A randomised open-label controlled trial. Lancet 2014;384:1265-72.

771. Steineck I, Cederholm J, Eliasson B, Rawshani A, Eeg-Olofsson K, Svensson AM, et al. Insulin pump therapy, multiple daily injections, and cardiovascular mortality in 18,168 people with type 1 diabetes: Observational study. BMJ 2015;350:h3234.

772. Hammond P, Boardman S, Greenwood R. ABCD position paper on insulin pumps. Pract Diabetes Int 2006;23:395-400.

773. Heller S, Kozlovski P, Kurtzhals P. Insulin's $85^{\text {th }}$ anniversary - An enduring medical miracle. Diabetes Res Clin Pract 2007;78:149-58.

774. Kesavadev J. Use of insulin pump in type 1 diabetes mellitus. Diabetes Clin Case Series 2016;1:124.

775. Kesavadev J. Continuous insulin infusion systems in type 2 diabetes. J Assoc Physicians India 2011;59 Suppl:41-3.

776. Kesavadev J. Insulin pump therapy in pregnancy. J Pak Med Assoc 2016;66:S39-44.

777. Kesavadev J, Shankar A, Sadasrian Pillai PB, Saboo B, Joshi S, Krishnan $\mathrm{G}$, et al. CSII as an alternative therapeutic strategy for managing type 2 diabetes: Adding the Indian experience to a global perspective. Curr Diabetes Rev 2016;12:312-4.

778. Kesavadev J. Fasting in Ramadan with an insulin pump. J Pak Med Assoc 2015;65:S47-50.

779. Kesavadev J. Insulin Pump Therapy: How to Choose the Right Candidate. In: Madhu S, editor. RSSDI Diabetes Update 2016. $1^{\text {st }}$ ed. Jaypee Brothers Medical Publishers Ltd.; 2017.

780. American Diabetes Association. 1. Improving care and promoting health in populations: Standards of medical care in diabetes-2019. Diabetes Care 2019;42:S7-12.

781. Tricco AC, Ivers NM, Grimshaw JM, Moher D, Turner L, Galipeau J, et al. Effectiveness of quality improvement strategies on the management of diabetes: A systematic review and meta-analysis. Lancet 2012;379:2252-61.

782. Cebul RD, Love TE, Jain AK, Hebert CJ. Electronic health records and quality of diabetes care. N Engl J Med 2011;365:825-33.

783. Battersby M, von Korff M, Schaefer J, Davis C, Ludman E, Greene SM, et al. Twelve evidence-based principles for implementing self-management support in primary care. Jt Comm J Qual Patient Saf 2010;36:561-70.

784. Jia P, Zhao P, Chen J, Zhang M. Evaluation of clinical decision support systems for diabetes care: An overview of current evidence. J Eval Clin Pract 2019;25:66-77.

785. Bright TJ, Wong A, Dhurjati R, Bristow E, Bastian L, Coeytaux RR, et al. Effect of clinical decision-support systems: A systematic review. Ann Intern Med 2012;157:29-43.

786. American Telemedicine Association. About Telemedicine. Available from: http://www.americantelemed.org/main/about/abouttelemedicine/telemedicine-faqs. [Last accessed on 2019 Mar 01].

787. Xu T, Pujara S, Sutton S, et al. Telemedicine in the management of type 1 diabetes. Prev Chronic Dis 2018;15:E13. doi: 10.5888/ pcd15.170168.

788. Lee SW, Chan CK, Chua SS, Chaiyakunapruk N. Comparative effectiveness of telemedicine strategies on type 2 diabetes management: A systematic review and network meta-analysis. Sci Rep 2017;7:12680.

789. Marcolino MS, Maia JX, Alkmim MB, Boersma E, Ribeiro AL. Telemedicine application in the care of diabetes patients: Systematic review and meta-analysis. PLoS One 2013;8:e79246. 
790. Heitkemper EM, Mamykina L, Travers J, Smaldone A. Do health information technology self-management interventions improve glycemic control in medically underserved adults with diabetes? A systematic review and meta-analysis. J Am Med Inform Assoc 2017;24:1024-35.

791. Kesavadev J, Shankar A, Pillai PB, Krishnan G, Jothydev S. Cost-effective use of telemedicine and self-monitoring of blood glucose via Diabetes Tele Management System (DTMS) to achieve target glycosylated hemoglobin values without serious symptomatic hypoglycemia in 1,000 subjects with type 2 diabetes mellitus-a retrospective study. Diabetes Technol Ther 2012;14:772-6.

792. Kesavadev J, Saboo B, Shankar A, Krishnan G, Jothydev S. Telemedicine for diabetes care: An Indian perspective - Feasibility and efficacy. Indian J Endocrinol Metab 2015;19:764-9.

793. Vitale RJ, Pillai PB, Krishnan G, Jothydev S, Kesavadev J. The two levels of care for diabetes in a developing country: Mechanisms for improved intermediate health outcomes. Diabetes Metab Syndr 2016;10:S90-4

794. mHealth: Mobile Technology Poised to Enable a New Era in Health Care. Ernst \& Young; 2012.

795. Shah VN, Garg SK. Managing diabetes in the digital age. Clin Diabetes Endocrinol 2015;1:16.

796. Krošel M, Švegl L, Vidmar L, et al. Empowering Diabetes Patient with Mobile Health Technologies. Mobile Health Technologies-Theories and Applications: InTech; 2016.

797. Healthline Media. The Best Diabetes Apps of; 2018. Available from: https://www.healthline.com/health/diabetes/top-iphone-androidapps\#diabetes. [Last accessed on 2019 Mar 01].

798. Dhatariya K, Levy N, Kilvert A, Watson B, Cousins D, Flanagan D, et al. NHS Diabetes guideline for the perioperative management of the adult patient with diabetes. Diabet Med 2012;29:420-33.

799. Sebranek JJ, Lugli AK, Coursin DB. Glycaemic control in the perioperative period. Br J Anaesth 2013;111 Suppl 1:i18-34.

800. Kuzulugil D, Papeix G, Luu J, Kerridge RK. Recent advances in diabetes treatments and their perioperative implications. Curr Opin Anaesthesiol 2019;32:398-404

801. Lin CS, Chang CC, Lee YW, Liu CC, Yeh CC, Chang YC, et al. Adverse outcomes after major surgeries in patients with diabetes: A multicenter matched study. J Clin Med 2019;8. pii: E100.

802. Dagogo-Jack S, Alberti KG. Management of Diabetes mellitus in surgical patients. Diabetes Spectrum 2002;15:44-8.

803. Gupta S. Preoperative Management of the Patient with Diabetes.

804. Setji TL, Gilmore L, Freeman S. Perioperative diabetes management. Diabetes Manag 2018;8:67-73.

805. Sudhakaran S, Surani SR. Guidelines for perioperative management of the diabetic patient. Surg Res Pract 2015;2015:284063.

806. Leung V, Ragbir-Toolsie K. Perioperative management of patients with diabetes. Health Serv Insights 2017;10:1178632917735075.

807. American Diabetes Association. 15. Diabetes care in the hospital: Standards of medical care in diabetes-2019. Diabetes Care 2019;42:S173-81.

808. Azoulay L, Suissa S. Sulfonylureas and the risks of cardiovascular events and death: A methodological meta-regression analysis of the observational studies. Diabetes Care 2017;40:706-14.

809. Meneghini LF. Perioperative management of diabetes: Translating evidence into practice. Cleve Clin J Med 2009;76 Suppl 4:S53-9.

810. Meyer EJ, Gabb G, Jesudason D. SGLT2 inhibitor-associated euglycemic diabetic ketoacidosis: A South Australian clinical case series and Australian spontaneous adverse event notifications. Diabetes Care 2018;41:e47-9.

811. Burke KR, Schumacher CA, Harpe SE. SGLT2 Inhibitors: A systematic review of diabetic ketoacidosis and related risk factors in the primary literature. Pharmacotherapy 2017;37:187-94.

812. Umpierez GE. How to manage type 2 diabetes in medical and surgical patients in the hospital. Cleve Clin J Med 2011;78:379-84

813. American Diabetes Association. 14. Diabetes care in the hospital: Standards of medical care in diabetes-2018. Diabetes Care 2018;41:S144-51

814. Polderman JA, van Steen SC, Thiel B, Godfried MB, Houweling PL, Hollmann MW, et al. Peri-operative management of patients with type-2 diabetes mellitus undergoing non-cardiac surgery using liraglutide, glucose-insulin-potassium infusion or intravenous insulin bolus regimens: A randomised controlled trial. Anaesthesia 2018;73:332-9.

815. Abuannadi M, Kosiborod M, Riggs L, House JA, Hamburg MS, Kennedy KF, et al. Management of hyperglycemia with the administration of intravenous exenatide to patients in the cardiac intensive care unit. Endocr Pract 2013;19:81-90.

816. Toeller M. alpha-Glucosidase inhibitors in diabetes: Efficacy in NIDDM subjects. Eur J Clin Invest 1994;24 Suppl 3:31-5.

817. Vann MA. Perioperative management of ambulatory surgical patients with diabetes mellitus. Curr Opin Anaesthesiol 2009;22:718-24

818. Dobri GA, Lansang MC. Q: How should we manage insulin therapy before surgery? Cleve Clin J Med 2013;80:702-4.

819. Joshi GP, Chung F, Vann MA, Ahmad S, Gan TJ, Goulson DT, et al. Society for Ambulatory Anesthesia consensus statement on perioperative blood glucose management in diabetic patients undergoing ambulatory surgery. Anesth Analg 2010;111:1378-87.

820. Evers IM, de Valk HW, Visser GH. Risk of complications of pregnancy in women with type 1 diabetes: Nationwide prospective study in the Netherlands. BMJ 2004;328:915.

821. Lawrence JM, Contreras R, Chen W, Sacks DA. Trends in the prevalence of preexisting diabetes and gestational diabetes mellitus among a racially/ethnically diverse population of pregnant women, 1999-2005. Diabetes Care 2008;31:899-904.

822. Renji; SR, Lekshmi ST, Chellamma N. Prevalence of pre-gestational diabetes among the antenatal women attending a tertiary care center. Int J Reprod Contracept Obstret Gynecol 2017;6:797-801.

823. Ramadevi V. Prevalence of pre-gestational diabetes in pregnancy. MedPulse Int J Gynaecol 2017;3:80-3.

824. Wahabi HA, Esmaeil SA, Fayed A, Al-Shaikh G, Alzeidan RA. Pre-existing diabetes mellitus and adverse pregnancy outcomes. BMC Res Notes 2012;5:496.

825. Negrato CA, Mattar R, Gomes MB. Adverse pregnancy outcomes in women with diabetes. Diabetol Metab Syndr 2012;4:41.

826. Yu L, Zeng XL, Cheng ML, et al. Quantitative assessment of the effect of pre-gestational diabetes and risk of adverse maternal, perinatal and neonatal outcomes. Oncotarget 2017;8:61048-56.

827. Kalra B, Gupta Y, Kalra S. Pre-conception management of diabetes. J Pak Med Assoc 2015;65:1242-3.

828. Yehuda I. Implementation of preconception care for women with diabetes. Diabetes spectrum: A publication of the American Diabetes Association 2016;29:105-14.

829. American Diabetes Association. Preconception care of women with diabetes. Diabetes Care 2004;27 Suppl 1:S76-8

830. American Diabetes Association. 14. Management of diabetes in pregnancy: Standards of medical care in diabetes-2019. Diabetes Care 2019;42:S165-72.

831. National Institue for Health Care and Excellence. Diabetes in pregnancy: Management from Preconception to the Postnatal Period; 2015. Available from: https://www.nice.org.uk/guidance/ng3. [Last accessed on 19 Aug 20]

832. Kitzmiller JL, Block JM, Brown FM, Catalano PM, Conway DL, Coustan DR, et al. Managing preexisting diabetes for pregnancy: Summary of evidence and consensus recommendations for care. Diabetes Care 2008;31:1060-79.

833. Buschur ES, Barbour LA. Diabetes in Pregnancy. Diabetes in Pregnancy: Endotext. Available from: https://www.ncbi.nlm.nih.gov/ books/NBK279010/. [Last accessed on 2019 Aug 10, Last updated 2018 Jan].

834. ACOG Practice Bulletin No. 201: Pregestational diabetes mellitus. Obstet Gynecol 2018;132:e228-48.

835. Fong DS, Aiello L, Gardner TW, King GL, Blankenship G, Cavallerano JD, et al. Retinopathy in diabetes. Diabetes Care 2004;27 Suppl 1:S84-7.

836. Young EC, Pires ML, Marques LP, de Oliveira JE, Zajdenverg L. Effects of pregnancy on the onset and progression of diabetic nephropathy and of diabetic nephropathy on pregnancy outcomes. Diabetes Metab Syndr 2011;5:137-42.

837. Blumer I, Hadar E, Hadden DR, Jovanovič L, Mestman JH, Murad MH, 
et al. Diabetes and pregnancy: An endocrine society clinical practice guideline. J Clin Endocrinol Metab 2013;98:4227-49.

838. Bryant SN, Herrera CL, Nelson DB, Cunningham FG. Diabetic ketoacidosis complicating pregnancy. J Neonatal Perinatal Med 2017; 10:17-23.

839. Magee LA, von Dadelszen P, Rey E, Ross S, Asztalos E, Murphy KE, et al. Less-tight versus tight control of hypertension in pregnancy. $\mathrm{N}$ Engl J Med 2015;372:407-17.

840. Wild R, Weedin EA, Gill EA. Women's health considerations for lipid management. Cardiol Clin 2015;33:217-31.

841. Lambert CT, Sandesara P, Isiadinso I; Gongora MC, Eapen D, Bhatia N, et al. Current Treatment of Familial Hypercholesterolaemia. Eur Cardiol 2014;9:76-81.

842. Thorogood M, Seed M, De Mott K; Guideline Development Group. Management of fertility in women with familial hypercholesterolaemia: Summary of NICE guidance. BJOG 2009;116:478-9.

843. Correa A, Gilboa SM, Botto LD, Moore CA, Hobbs CA, Cleves MA, et al. Lack of periconceptional vitamins or supplements that contain folic acid and diabetes mellitus-associated birth defects. Am J Obstet Gynecol 2012;206:218.e1-13.

844. Rahman D, Shahida SM, Hussain T, Begum A. Orlistat (the lipase inhibitor) therapy in overweight and obese subfertile women. Mymensingh Med J 2017;26:432-8.

845. Behl S, Misra A. Management of obesity in adult Asian Indians. Indian Heart J 2017;69:539-44.

846. Kalra P, Anakal M. Peripartum management of diabetes. Indian J Endocrinol Metab 2013;17:S72-6.

847. Barrett HL, Morris J, McElduff A. Watchful waiting: A management protocol for maternal glycaemia in the peripartum period. Aust N Z J Obstet Gynaecol 2009;49:162-7.

848. Mitanchez D, Yzydorczyk C, Simeoni U. What neonatal complications should the pediatrician be aware of in case of maternal gestational diabetes? World J Diabetes 2015;6:734-43.

849. Parks C, Peipert JF. Eliminating health disparities in unintended pregnancy with long-acting reversible contraception (LARC). Am J Obstet Gynecol 2016;214:681-8.

850. Dharmalingam M. Glycemic control in Intensive Care Unit. Indian J Endocrinol Metab 2016;20:415-7.

851. Boord JB, Graber AL, Christman JW, Powers AC. Practical management of diabetes in critically ill patients. Am J Respir Crit Care Med 2001;164:1763-7.

852. Prieto-Sanchez LM. Hyperglycemia in-hospital management. Ther Adv Endocrinol Metab 2011;2:3-7.

853. Bajwa SJ, Baruah MP, Kalra S, Kapoor MC. Interdisciplinary position statement on management of hyperglycemia in peri-operative and intensive care. J Anaesthesiol Clin Pharmacol 2015;31:155-64.

854. Moghissi ES, Korytkowski MT, DiNardo M, Einhorn D, Hellman R, Hirsch IB, et al. American Association of Clinical Endocrinologists and American Diabetes Association consensus statement on inpatient glycemic control. Diabetes Care 2009;32:1119-31.

855. Qaseem A, Chou R, Humphrey LL, Shekelle P; Clinical Guidelines Committee of the American College of Physicians. Inpatient glycemic control: Best practice advice from the Clinical Guidelines Committee of the American College of Physicians. Am J Med Qual 2014;29:95-8.

856. Gardner AJ. The benefits of tight glycemic control in critical illness: Sweeter than assumed? Indian J Crit Care Med 2014;18:807-13.

857. NICE-SUGAR Study Investigators, Finfer S, Chittock DR, Su SY, Blair D, Foster D, et al. Intensive versus conventional glucose control in critically ill patients. N Engl J Med 2009;360:1283-97.

858. Clement S, Braithwaite SS, Magee MF, Ahmann A, Smith EP, Schafer RG, et al. Management of diabetes and hyperglycemia in hospitals. Diabetes Care 2004;27:553-91.

859. Krinsley JS, Chase JG, Gunst J, Martensson J, Schultz MJ, Taccone FS, et al. Continuous glucose monitoring in the ICU: Clinical considerations and consensus. Crit Care 2017;21:197.

860. Umpierrez GE, Klonoff DC. Diabetes technology update: Use of insulin pumps and continuous glucose monitoring in the hospital. Diabetes Care 2018;41:1579-89.

861. Narayan KM, Fagot-Campagna A, Imperatore G. Type 2 diabetes in children: A problem lurking for India? Indian Pediatr 2001;38:701-4.
862. Bhatia V, Arya V, Dabadghao P, Balasubramanian K, Sharma K, Verghese $\mathrm{N}$, et al. Etiology and outcome of childhood and adolescent diabetes mellitus in North India. J Pediatr Endocrinol Metab 2004;17:993-9.

863. Mohan V, Deepa M, Deepa R, Shanthirani CS, Farooq S, Ganesan A, et al. Secular trends in the prevalence of diabetes and impaired glucose tolerance in urban South India-the Chennai Urban Rural Epidemiology Study (CURES-17). Diabetologia 2006;49:1175-8.

864. Mohan V, Sandeep S, Deepa R, Shah B, Varghese C. Epidemiology of type 2 diabetes: Indian scenario. Indian J Med Res 2007;125:217-30.

865. Ramachandran A, Mohan V, Snehalatha C, Bharani G, Chinnikrishnudu M, Mohan R, et al. Clinical features of diabetes in the young as seen at a diabetes centre in south India. Diabetes Res Clin Pract 1988;4:117-25.

866. Ramachandran A, Snehalatha C, Kapur A, Vijay V, Mohan V, Das AK, et al. High prevalence of diabetes and impaired glucose tolerance in India: National Urban Diabetes Survey. Diabetologia 2001;44:1094-101

867. Ramachandran A, Snehalatha C, Satyavani K, Sivasankari S, Vijay V. Type 2 diabetes in Asian-Indian urban children. Diabetes Care 2003;26:1022-5.

868. Deepa R, Sandeep S, Mohan V. Abdominal Obesity Viceral Fat and Type 2 Diabetes-Asian Indian Phenotype; 2007.

869. Ramachandran A, Snehalatha C, Dharmaraj D, Viswanathan M. Prevalence of glucose intolerance in Asian Indians. Urban-rural difference and significance of upper body adiposity. Diabetes Care 1992;15:1348-55.

870. Ramachandran A, Snehalatha C, Latha E, Manoharan M, Vijay V. Impacts of urbanisation on the lifestyle and on the prevalence of diabetes in native Asian Indian population. Diabetes Res Clin Pract 1999;44:207-13

871. Yajnik CS. The insulin resistance epidemic in India: Fetal origins, later lifestyle, or both? Nutr Rev 2001;59:1-9.

872. Yajnik CS, Fall CH, Coyaji KJ, Hirve SS, Rao S, Barker DJ, et al. Neonatal anthropometry: The thin-fat Indian baby. The pune maternal nutrition study. Int J Obes Relat Metab Disord 2003;27:173-80.

873. Lascar N, Brown J, Pattison H, Barnett AH, Bailey CJ, Bellary S. Type 2 diabetes in adolescents and young adults. Lancet Diabetes Endocrinol 2018;6:69-80.

874. TODAY Study Group. Effects of metformin, metformin plus rosiglitazone, and metformin plus lifestyle on insulin sensitivity and $\beta$-cell function in TODAY. Diabetes Care 2013;36:1749-57.

875. Reinehr T. Type 2 diabetes mellitus in children and adolescents. World J Diabetes 2013;4:270-81.

876. Screening for type 2 diabetes in children and adolescents. AAP Grand Rounds 2017:37:35-5.

877. Wittmeier KD, Wicklow BA, Sellers EA, et al. Success with lifestyle monotherapy in youth with new-onset type 2 diabetes. Paediat Child Health 2012;17:129-32.

878. Kautiainen S, Koivusilta L, Lintonen T, Virtanen SM, Rimpelä A. Use of information and communication technology and prevalence of overweight and obesity among adolescents. Int $\mathrm{J}$ Obes (Lond) 2005;29:925-33.

879. TODAY Study Group, Zeitler P, Hirst K, Pyle L, Linder B, Copeland K, et al. A clinical trial to maintain glycemic control in youth with type 2 diabetes. N Engl J Med 2012;366:2247-56.

880. Mingrone G. Pros and cons of bariatric surgery in adolescents. Lancet Diabetes Endocrinol 2017;5:152-4.

881. Persynaki A, Karras S, Pichard C. Unraveling the metabolic health benefits of fasting related to religious beliefs: A narrative review. Nutrition 2017;35:14-20.

882. Julka S, Sachan A, Bajaj S, Sahay R, Chawla R, Agrawal N, et al. Glycemic management during Jain fasts. Indian J Endocrinol Metab 2017;21:238-41.

883. Al-Arouj M, Assaad-Khalil S, Buse J, Fahdil I, Fahmy M, Hafez S, et al. Recommendations for management of diabetes during Ramadan: Update 2010. Diabetes Care 2010;33:1895-902.

884. Bashir MI, Pathan MF, Raza SA, Ahmad J, Khan AK, Ishtiaq O, et al. Role of oral hypoglycemic agents in the management of type 2 diabetes mellitus during Ramadan. Indian J Endocrinol Metab 2012;16:503-7.7. 
885. Bajaj S. Newer antidiabetic drugs in Ramadan. J Pak Med Assoc 2015;65:S40-3.

886. Hassanein M, Al-Arouj M, Hamdy O, Bebakar WM, Jabbar A, AlMadani A, et al. Diabetes and Ramadan: Practical guidelines. Diabetes Res Clin Pract 2017;126:303-16.

887. Diabetes and Ramadan: Practical Guidelines. International Diabetes Federation; 2016. Available from: https://www.idf.org/e-library/ guidelines/87-diabetes-and-ramadan-practical-25. [Last accessed on 2016 Jun 19]

888. Kalra S, Bajaj S, Gupta Y, Agarwal P, Singh SK, Julka S, et al. Fasts, feasts and festivals in diabetes-1: Glycemic management during Hindu fasts. Indian J Endocrinol Metab 2015;19:198-203.

889. Latt T, SK. Managing diabetes during fasting-A focus on Buddhist Lent. Diabetes Voice 2012;57:42-5.

890. Zainudin SB, Ang DY, Soh AW. Knowledge of diabetes mellitus and safe practices during Ramadan fasting among Muslim patients with diabetes mellitus in Singapore. Singapore Med J 2017;58:246-52.

891. Norouzy A, Mohajeri SM, Shakeri S, Yari F, Sabery M, Philippou E, et al. Effect of Ramadan fasting on glycemic control in patients with Type 2 diabetes. J Endocrinol Invest 2012;35:766-71.

892. Almansour HA, Chaar B, Saini B. Fasting, diabetes, and optimizing health outcomes for Ramadan observers: A literature review. Diabetes Ther 2017;8:227-49.

893. Jabbar A. Glucose monitoring during Ramadan. J Pak Med Assoc 2015;65:S51-3.

894. McEwen LN, Ibrahim M, Ali NM, Assaad-Khalil SH, Tantawi HR, Nasr G, et al. Impact of an individualized type 2 diabetes education program on clinical outcomes during Ramadan. BMJ Open Diabetes Res Care 2015;3:e00111.

895. Buchmann M, Wermeling M, Lucius-Hoene G, Himmel W. Experiences of food abstinence in patients with type 2 diabetes: A qualitative study. BMJ Open 2016;6:e008907.

896. Ali S, Davies MJ, Brady EM, Gray LJ, Khunti K, Beshyah SA, et al. Guidelines for managing diabetes in Ramadan. Diabet Med 2016;33:1315-29.

897. Almalki MH, Alshahrani F. Options for controlling type 2 diabetes during Ramadan. Front Endocrinol (Lausanne) 2016;7:32

898. Niazi AK, Kalra S. Patient centred care in diabetology: An Islamic perspective from South Asia. J Diabetes Metab Disord 2012;11:30.

899. Zargar AH, Siraj M, Jawa AA, Hasan M, Mahtab H. Maintenance of glycaemic control with the evening administration of a long acting sulphonylurea in male type 2 diabetic patients undertaking the Ramadan fast. Int J Clin Pract 2010;64:1090-4.

900. Gray LJ, Dales J, Brady EM, Khunti K, Hanif W, Davies MJ. Safety and effectiveness of non-insulin glucose-lowering agents in the treatment of people with type 2 diabetes who observe Ramadan: A systematic review and meta-analysis. Diabetes Obes Metab 2015;17:639-48.

901. Aravind SR, Ismail SB, Balamurugan R, Gupta JB, Wadhwa T, Loh SM, et al. Hypoglycemia in patients with type 2 diabetes from India and Malaysia treated with sitagliptin or a sulfonylurea during Ramadan: A randomized, pragmatic study. Curr Med Res Opin 2012;28:1289-96.

902. Brady EM, Davies MJ, Gray LJ, Saeed MA, Smith D, Hanif W, et al. A randomized controlled trial comparing the GLP-1 receptor agonist liraglutide to a sulphonylurea as add on to metformin in patients with established type 2 diabetes during Ramadan: The Treat 4 Ramadan Trial. Diabetes Obes Metab 2014;16:527-36.

903. Azar ST, Echtay A, Wan Bebakar WM, Al Araj S, Berrah A, Omar M, et al. Efficacy and safety of liraglutide compared to sulphonylurea during Ramadan in patients with type 2 diabetes (LIRA-Ramadan): A randomized trial. Diabetes Obes Metab 2016;18:1025-33.

904. Wan Seman WJ, Kori N, Rajoo S, Othman H, Mohd Noor N, Wahab NA, et al. Switching from sulphonylurea to a sodium-glucose cotransporter2 inhibitor in the fasting month of Ramadan is associated with a reduction in hypoglycaemia. Diabetes Obes Metab 2016;18:628-32.

905. Beshyah SA, Chatterjee S, MJ. Use of SGLT2 inhibitors during Ramadan: A survey of physicians' views and practical guidance $\mathrm{Br} \mathrm{J}$ Diabetes 2016;16:20-24

906. Kalra S, Jawad F. Insulin in Ramadan. J Pak Med Assoc 2015;65:S44-6.
907. Chrvala CA, Sherr D, Lipman RD. Diabetes self-management education for adults with type 2 diabetes mellitus: A systematic review of the effect on glycemic control. Patient Educ Couns 2016;99:926-43.

908. Scain SF, Friedman R, Gross JL. A structured educational program improves metabolic control in patients with type 2 diabetes: A randomized controlled trial. Diabetes Educ 2009;35:603-11.

909. Horigan G, Davies M, Findlay-White F, Chaney D, Coates V. Reasons why patients referred to diabetes education programmes choose not to attend: A systematic review. Diabet Med 2017;34:14-26.

910. Mulcahy K, Maryniuk M, Peeples M, Peyrot M, Tomky D, Weaver T, et al. Diabetes self-management education core outcomes measures. Diabetes Educ 2003;29:768-70, 773-84, 787-8 passim.

911. Summary of Revisions: Standards of medical care in diabetes-2018. Diabetes Care 2018;41:S4-6.

912. Deepa M, Bhansali A, Anjana RM, Pradeepa R, Joshi SR, Joshi PP, et al. Knowledge and awareness of diabetes in urban and rural India: The Indian Council of Medical Research India Diabetes Study (Phase I): Indian Council of Medical Research India Diabetes 4. Indian J Endocrinol Metab 2014;18:379-85.

913. Ramachandran A, Snehalatha C, Baskar AD, Mary S, Kumar CK, Selvam S, et al. Temporal changes in prevalence of diabetes and impaired glucose tolerance associated with lifestyle transition occurring in the rural population in India. Diabetologia 2004;47:860-5.

914. Singh DK, Tari V. Structured diabetes care (Freedom $365^{*}$ ) provides better glycemic control than routine medical care in type 2 diabetes: Proof of concept observational study. Int J Diabetes Dev Ctries 2015;35:289-96

915. Jankowska-Polańska B, Fal AM, Uchmanowicz I. Influence of organized diabetic education on self-control and quality of life of patients with type 2 diabetes. Int J Diabetes Dev Ctries 2015;35:79-87.

916. Verma R, Khanna P, Mehta B. National programme on prevention and control of diabetes in India: Need to focus. Australas Med J 2012;5:310-5.

917. Hussain R, Rajesh B, Giridhar A, Gopalakrishnan M, Sadasivan S, James $\mathrm{J}$, et al. Knowledge and awareness about diabetes mellitus and diabetic retinopathy in suburban population of a South Indian state and its practice among the patients with diabetes mellitus: A population-based study. Indian J Ophthalmol 2016;64:272-6.

918. Devi R, Kapoor B, Singh MM. Effectiveness of self-learning module on the knowledge and practices regarding foot care among type II diabetes patients in East Delhi, India. Int J Community Med Public Health 2017;3:2133-41.

919. Kide S, Rangari A, Shiral R. Knowledge and awareness of diabetes amongst diabetes patients in Wardha region. Int J Diabetes Dev Ctries 2014;34:232-2

920. Limaye TY, Wagle SS, Kumaran K. Lack of knowledge about diabetes in Pune-the city of knowledge! Int J Diabetes Dev Ctries 2016;36:26370

921. Long P, Long KN, Kedia A. A cross-sectional study of diabetic knowledge in West Bengal, India: An analysis based on access to health care. Int J Diabetes Dev Ctries 2015;35:614-9.

922. Mathew AC, Jacob N, Jose S. Knowledge about risk factors, symptoms and complications of diabetes among adults in South India. Int J Med Sci Public Health 2014;3:1086-93.

923. Muninarayana C, Balachandra G, Hiremath SG, Iyengar K, Anil NS. Prevalence and awareness regarding diabetes mellitus in rural Tamaka, Kolar. Int J Diabetes Dev Ctries 2010;30:18-21.

924. Saurabh S, Sarkar S, Selvaraj K, Kar SS, Kumar SG, Roy G. Effectiveness of foot care education among people with type 2 diabetes in rural Puducherry, India. Indian J Endocrinol Metab 2014;18:106-10.

925. Singh AK, Mani K, Krishnan A, Aggarwal P, Gupta SK. Prevalence, awareness, treatment and control of diabetes among elderly persons in an urban slum of delhi. Indian J Community Med 2012;37:236-9.

926. Jaiswal K, Moghe N, Mehta M, et al. Knowledge, attitude \& practices of type II diabetes mellitus patients in a tertiary care teaching institute of central India. J Diabetes Metab Dis Control 2019;6:1-4.

927. Sharma S, Jha J, Varshney A, et al. Awareness of various aspects of diabetes among people visiting tertiary eye care institute in north India. Clin Epidemiol Global Health 2019.

928. Bansal C, Kaushik R. Awareness of diabetic nephropathy in patients 
with type 2 diabetes mellitus: The Indian scenario. J Nephropharmacol 2018;7:90-7.

929. Krishnan A, Gupta V, Ritvik , Nongkynrih B, Thakur J. How to Effectively Monitor and Evaluate NCD Programmes in India. Indian J Community Med 2011;36:S57-62.

930. Mahajan PB. Role of medical colleges in prevention and control of diabetes in India: A ten point approach. Int J Diabetes Dev Ctries 2011;31:41-2.

931. Venkataraman K, Kannan AT, Mohan V. Challenges in diabetes management with particular reference to India. Int J Diabetes Dev Ctries 2009;29:103-9.

932. Basu S, Sharma N. Diabetes self-care in primary health facilities in India - challenges and the way forward. World $\mathrm{J}$ Diabetes 2019; 10:341-9.

933. Gupta K, Gupta S. Barriers to insulin therapy. J Diabetes Educ 2013b;3:17-23.

934. Bhojani U, Kolsteren P, Criel B, de Henauw S, Beerenahally TS, Verstraeten $\mathrm{R}$, et al. Intervening in the local health system to improve diabetes care: Lessons from a health service experiment in a poor urban neighborhood in India. Glob Health Action 2015;8:28762.

935. Basu S, Garg S. The barriers and challenges toward addressing the social and cultural factors influencing diabetes self-management in Indian populations. J Soc Health Diabetes 2017;5:071-6

936. Babu MS, Gowdappa HB, Kalpana T, Vidyalaxmi K, Nikhil B, Chakravarthy T. Knowledge, attitude and practices of diabetic patients - A cross sectional study in a tertiary care hospital in Mysore. J Assoc Physicians India 2015;63:96.

937. Chandalia HB, Modi S. Counseling strategies (Dr. Vinod Dhurandhar Oration at AIAAROCON-Pune on $9^{\text {th }}$ February, 2013). J Obes Metab Res 2014:1:43.

938. Hasan H, Zodpey S, Saraf A. Diabetes care in India: Assessing the need for Evidence-Based education. South-East Asian J Med Educ 2011;5:15-8.

939. Madhu S, Lalitha K. Education needs of Diabetic Patients with Low Socio-Economic and Literacy Levels, $15^{\text {th }}$ International Diabetes Federation (IDF) Congress. Kobe, Japan: Published in Scientific Supplement; 1994. p. 137.

940. Siaw MYL, Ko Y, Malone DC, Tsou KYK, Lew YJ, Foo D, et al. Impact of pharmacist-involved collaborative care on the clinical, humanistic and cost outcomes of high-risk patients with type 2 diabetes (IMPACT): A randomized controlled trial. J Clin Pharm Ther 2017;42:475-82.

941. Gonzalvo JD, Hamm J, Eaves S, et al. A practical approach to mental health for the diabetes educator. AADE Pract 2019;7:29-44.

942. Ciechanowski PS, Katon WJ, Russo JE. Depression and diabetes: Impact of depressive symptoms on adherence, function, and costs. Arch Intern Med 2000;160:3278-85.

943. Kalra S, Jena BN, Yeravdekar R. Emotional and psychological needs of people with diabetes. Indian J Endocrinol Metab 2018;22:696-704.

944. Pouwer F, Beekman AT, Nijpels G, Dekker JM, Snoek FJ, Kostense PJ, et al. Rates and risks for co-morbid depression in patients with Type 2 diabetes mellitus: Results from a community-based study. Diabetologia 2003:46:892-8

945. Schram MT, Baan CA, Pouwer F. Depression and quality of life in patients with diabetes: A systematic review from the European depression in diabetes (EDID) research consortium. Curr Diabetes Rev 2009:5:112-9.

946. Madhu K. Psychological and Psychiatric Aspects of Diabetes. Springer; 2015

947. Petrak F, Herpertz S, Albus C, Hirsch A, Kulzer B, Kruse J. Psychosocial factors and diabetes mellitus: Evidence-based treatment guidelines. Curr Diabetes Rev 2005;1:255-70.

948. Sridhar G, Madhu K. Psychosocial and cultural issues in diabetes mellitus. Current Science. 2002:1556-64

949. Kalra S, Balhara YPS, Bathla M. Euthymia in diabetes. Eur Endocrinol 2018;14:18-9.

950. Kalra S, Das AK, Baruah MP, Unnikrishnan AG, Dasgupta A, Shah P, et al. Euthymia in diabetes: Clinical evidence and practice-based opinion from an international expert group. Diabetes Ther 2019:10:791-804.
951. American Diabetes Association. 5. Lifestyle management: Standards of medical care in diabetes-2019. Diabetes Care 2019;42:S46-60.

952. Peyrot M, Rubin RR, Lauritzen T, Snoek FJ, Matthews DR, Skovlund SE. Psychosocial problems and barriers to improved diabetes management: Results of the Cross-National Diabetes Attitudes, Wishes and Needs (DAWN) Study. Diabet Med 2005;22:1379-85.

953. Powers MA, Bardsley J, Cypress M, et al. Diabetes self-management education and support in type 2 diabetes: A joint position statement of the American Diabetes Association, the American Association of Diabetes Educators, and the Academy of Nutrition and Dietetics. Diabetes Educ 2017;43:40-53

954. Bhardwaj S, Misra A. Obesity, diabetes and the Asian phenotype. World Rev Nutr Diet 2015;111:116-22.

955. Handelsman Y, Mechanick JI, Blonde L, Grunberger G, Bloomgarden ZT, Bray GA, et al. American Association of Clinical Endocrinologists Medical Guidelines for clinical practice for developing a diabetes mellitus comprehensive care plan: Executive summary. Endocr Pract 2011;17:287-302.

956. Bonsignore M, Barkow K, Jessen F, Heun R. Validity of the five-item WHO Well-Being Index (WHO-5) in an elderly population. Eur Arch Psychiatry Clin Neurosci 2001;251 Suppl 2:II27-31.

957. Kalra S, Sridhar GR, Balhara YP, Sahay RK, Bantwal G, Baruah MP, etal. National recommendations: Psychosocial management of diabetes in India. Indian J Endocrinol Metab 2013;17:376-95.

958. Anderson RJ, Freedland KE, Clouse RE, Lustman PJ. The prevalence of comorbid depression in adults with diabetes: A meta-analysis. Diabetes Care 2001;24:1069-78

959. Mezuk B, Eaton WW, Albrecht S, Golden SH. Depression and type 2 diabetes over the lifespan: A meta-analysis. Diabetes Care 2008;31:2383-90.

960. Young-Hyman DL, Davis CL. Disordered eating behavior in individuals with diabetes: Importance of context, evaluation, and classification. Diabetes Care 2010;33:683-9.

961. Khaledi M, Haghighatdoost F, Feizi A, Aminorroaya A. The prevalence of comorbid depression in patients with type 2 diabetes: An updated systematic review and meta-analysis on huge number of observational studies. Acta Diabetol 2019;56:631-50.

962. Egede LE, Zheng D, Simpson K. Comorbid depression is associated with increased health care use and expenditures in individuals with diabetes. Diabetes Care 2002;25:464-70.

963. Lin EH, Katon W, Von Korff M, Rutter C, Simon GE, Oliver M, et al. Relationship of depression and diabetes self-care, medication adherence, and preventive care. Diabetes Care 2004;27:2154-60.

964. Fisher L, Hessler D, Glasgow RE, Arean PA, Masharani U, Naranjo D, et al. REDEEM: A pragmatic trial to reduce diabetes distress. Diabetes Care 2013;36:2551-8.

965. Huang Y, Wei X, Wu T, Chen R, Guo A. Collaborative care for patients with depression and diabetes mellitus: A systematic review and meta-analysis. BMC Psychiatry 2013;13:260.

966. Ismail K, Winkley K, Rabe-Hesketh S. Systematic review and meta-analysis of randomised controlled trials of psychological interventions to improve glycaemic control in patients with type 2 diabetes. Lancet 2004:363:1589-97.

967. Lustman PJ, Griffith LS, Freedland KE, Kissel SS, Clouse RE. Cognitive behavior therapy for depression in type 2 diabetes mellitus. A randomized, controlled trial. Ann Intern Med 1998;129:613-21.

968. Snoek FJ, Skinner TC. Psychological counselling in problematic diabetes: Does it help? Diabet Med 2002;19:265-73.

969. Birdee GS, Yeh G. Complementary and alternative medicine therapies for diabetes: A clinical review. Clin Diabetes 2010;28:147-55.

970. Bhalerao MS, Bolshete PM, Swar BD, Bangera TA, Kolhe VR, Tambe MJ, et al. Use of and satisfaction with complementary and alternative medicine in four chronic diseases: A cross-sectional study from India. Natl Med J India 2013;26:75-8.

971. Kumar D, Bajaj S, Mehrotra R. Knowledge, attitude and practice of complementary and alternative medicines for diabetes. Public Health 2006;120:705-11.

972. Vishnu N, Mini GK, Thankappan KR. Complementary and alternative medicine use by diabetes patients in Kerala, India. Glob Health Epidemiol Genom 2017;2:e6. 
973. Fuangchan A, Sonthisombat P, Seubnukarn T, Chanouan R, Chotchaisuwat P, Sirigulsatien V, et al. Hypoglycemic effect of bitter melon compared with metformin in newly diagnosed type 2 diabetes patients. J Ethnopharmacol 2011;134:422-8.

974. Nakanekar A, Kohli K, Tatke P. Ayurvedic polyherbal combination (PDBT) for prediabetes: A randomized double blind placebo controlled study. J Ayurveda Integr Med 2019;10:284-9.

975. Sharma RD, Raghuram TC, Rao NS. Effect of fenugreek seeds on blood glucose and serum lipids in type I diabetes. Eur J Clin Nutr 1990;44:301-6.

976. Pandey A, Tripathi P, Pandey R, Srivatava R, Goswami S. Alternative therapies useful in the management of diabetes: A systematic review. J Pharm Bioallied Sci 2011;3:504-12.

977. Gupta RC, Chang D, Nammi S, Bensoussan A, Bilinski K, Roufogalis BD. Interactions between antidiabetic drugs and herbs: An overview of mechanisms of action and clinical implications. Diabetol Metab Syndr 2017;9:59.

978. Mills SY. Regulation in complementary and alternative medicine. BMJ 2001;322:158-60.

979. Myers SP, Cheras PA. The other side of the coin: Safety of complementary and alternative medicine. Med J Aust 2004;181:222-5.

980. Zhang, Yang L, Shergis J, Zhang L, Zhang AL, Guo X, et al. Chinese herbal medicine for diabetic kidney disease: A systematic review and meta-analysis of randomised placebo-controlled trials. BMJ Open 2019;9:e25653.

981. Ernst E. Toxic heavy metals and undeclared drugs in Asian herbal medicines. Trends Pharmacol Sci 2002;23:136-9.

982. Singh J, Singh R, Gautam CS. Self-medication with herbal remedies amongst patients of type 2 diabetes mellitus: A preliminary study. Indian J Endocrinol Metab 2012;16:662-3.

983. Nagarathna R, Usharani MR, Rao AR, et al. Efficacy of yoga based life style modification program on medication score and lipid profile in type 2 diabetes-a randomized control study. Int J Diabetes Dev Ctries 2012;32:122-30.

984. Aljasir B, Bryson M, Al-Shehri B. Yoga practice for the management of type II diabetes mellitus in adults: A systematic review. Evid Based Complement Alternat Med 2010;7:399-408.

985. Innes KE, Vincent HK. The influence of yoga-based programs on risk profiles in adults with type 2 diabetes mellitus: A systematic review. Evid Based Complement Alternat Med 2007;4:469-86.

986. Jyotsna VP, Joshi A, Ambekar S, Kumar N, Dhawan A, Sreenivas V. Comprehensive yogic breathing program improves quality of life in patients with diabetes. Indian J Endocrinol Metab 2012;16:423-8. 


\section{ANNEXURES}

\section{Screening/Early detection of diabetes}

\section{Annexure 1: The Indian Diabetes Risk Score (IDRS) ${ }^{[1-6]}$}

- The tool encompasses four parameters: age, abdominal obesity, family history of diabetes, and physical activity to detect T2DM and also helps to distinguish T2DM from non-T2DM.

- A maximum score of 100 is given for these categories combined as shown in the Table.

- Subjects with an IDRS of $<30$ are categorized under low risk, 30-50 as medium risk and those with $>60$ as high risk for diabetes.

- Similarly $\mathrm{WC} \geq 90 \mathrm{~cm}$, sedentary lifestyle and family history of diabetes are indicators for high risk of diabetes.

- Limiting the blood sugar testing to those with an IDRS score of 50 and above could identify more than $90 \%$ of Indians with diabetes and prediabetes.

\section{Indian Diabetes Risk Score}

\begin{tabular}{|c|c|}
\hline Parameter & Score \\
\hline \multicolumn{2}{|l|}{ Age (years) } \\
\hline$<35$ & 0 \\
\hline $35-49$ & 20 \\
\hline$\geq 50$ & 30 \\
\hline \multicolumn{2}{|l|}{$\mathrm{WC}$} \\
\hline Waist $<80 \mathrm{~cm}$ (female), $<90 \mathrm{~cm}$ (male) & 0 \\
\hline Waist $\geq 80-89 \mathrm{~cm}$ (female), $\geq 90-99 \mathrm{~cm}$ (male) & 10 \\
\hline Waist $\geq 90 \mathrm{~cm}$ (female), $\geq 100 \mathrm{~cm}$ (male) & 20 \\
\hline \multicolumn{2}{|l|}{ Physical activity } \\
\hline $\begin{array}{l}\text { Regular vigorous exercise or strenuous (manual) activity at } \\
\text { home/work }\end{array}$ & 0 \\
\hline $\begin{array}{l}\text { Regular moderate exercise or moderate physical activity at } \\
\text { home/work }\end{array}$ & 10 \\
\hline Regular mild exercise or mild physical activity at home/work & 20 \\
\hline No exercise and/or sedentary activities at home/work & 30 \\
\hline \multicolumn{2}{|l|}{ Family history of diabetes } \\
\hline No diabetes in parents & 0 \\
\hline One parent is diabetic & 10 \\
\hline Both parents are diabetic & 20 \\
\hline
\end{tabular}

\section{REFERENCES}

1. Mohan V, Sandeep S, Deepa M, Gokulakrishnan K, Datta M, Deepa R. A diabetes risk score helps identify metabolic syndrome and cardiovascular risk in Indians - The Chennai Urban Rural Epidemiology Study (CURES-38). Diabetes Obes Metab 2007;9:337-43.

2. Mohan V, Deepa M, Farooq S, Narayan KM, Datta M, Deepa R. Anthropometric cut points for identification of cardiometabolic risk factors in an urban Asian Indian population. Metabolism 2007;56:961-8.

3. Mohan V, Deepa M, Farooq S, Prabhakaran D, Reddy KS. Surveillance for risk factors of cardiovascular disease among an industrial population in southern India. Natl Med J India 2008;21:8-13.

4. Mohan V, Deepa M, Anjana RM, Lanthorn H, Deepa R. Incidence of diabetes and pre-diabetes in a selected urban south Indian population (CUPS-19). J Assoc Physicians India 2008;56:152-7.

5. Mohan V, Deepa R, Deepa M, Somannavar S, Datta M. A simplified Indian Diabetes Risk Score for screening for undiagnosed diabetic subjects. J Assoc Physicians India 2005;53:759-63.

6. Sharma KM, Ranjani H, Nguyen H, Shetty S, Datta M, Narayan KM, et al. Indian Diabetes Risk Score helps to distinguish type 2 from non-type 2 diabetes mellitus (GDRC-3). J Diabetes Sci Technol 2011;5:419-25. 
Annexure 2: Dr. A. Ramachandran's Diabetes Risk Score for Indians can be used to identify people having undetected diabetes. The likelihood of detecting diabetes in people with a score of 21 or more is high and require testing with an oral glucose tolerance test[1]

\begin{tabular}{lc}
\hline Variables & Risk score \\
\hline Age (years) & \\
$30-44$ & 10 \\
$45-59$ & 18 \\
$>59$ & 19 \\
Family history of diabetes & 7 \\
BMI $(\geq 25) \mathrm{kg} / \mathrm{m}^{2}$ & 7 \\
Waist $(\mathrm{M} \geq 85, \mathrm{~W} \geq 80) \mathrm{cm}$ & 5 \\
Sedentary physical activity & 4 \\
Maximum score & 42 \\
\hline
\end{tabular}

BMI: Body mass index

\section{REFERENCE}

1. Ramachandran A, Snehalatha C, Vijay V, Wareham NJ, Colagiuri S. Derivation and validation of diabetes risk score for urban Asian Indians. Diabetes Res Clin Pract 2005;70:63-70.

Annexure 3: Diabetic neuropathy symptom score (DNS) ${ }^{[1]}$

\section{DNS questionnaire}

1. Are you suffering of unsteadiness in walking?

Need for visual control, increase in dark, walk like a drunken man, and lack of contact with floor

2. Do you have a burning, aching pain or tenderness at your legs or feet? Occurring at rest or at night, not related to exercise, exclude intermittent claudication

3. Do you have prickling sensations at your legs and feet

Occurring at rest or at night, distal > proximal, stocking glove distribution

4. Do you have places of numbness on your legs or feet?

Distal $>$ proximal, stocking glove distribution

The questions were answered either "Yes" (positive: 1 point) if symptom has occurred during the last 2 weeks or 'No' (negative: 0 point) if it did not.

Maximum score is 4 and minimum 0 . DNS: Diabetic neuropathy symptom

\section{Reference}

1. Meijer JW, Smit AJ, Sonderen EV, Groothoff JW, Eisma WH, Links TP. Symptom scoring systems to diagnose distal polyneuropathy in diabetes: the Diabetic Neuropathy Symptom score. Diabet Med 2002;19:962-5.

\section{Annexure 4: Modified Neuropathy Disability Score (NDS) ${ }^{[1]}$}

\begin{tabular}{|c|c|}
\hline \multicolumn{2}{|l|}{ NDS } \\
\hline Vibration perception threshold & Normal $=0$ \\
\hline $\begin{array}{l}\text { 128-Hz tuning fork; apex of big toe: } \\
\text { Normal=Can distinguish vibrating/not vibrating }\end{array}$ & Abnormal $=1$ \\
\hline \multicolumn{2}{|l|}{ Temperature perception on dorsum of the foot } \\
\hline \multicolumn{2}{|l|}{ Use tuning fork with beaker of ice/warm water } \\
\hline \multicolumn{2}{|l|}{ Pin-prick } \\
\hline \multicolumn{2}{|l|}{$\begin{array}{l}\text { Apply pin proximal to big toe nail just enough } \\
\text { to deform the skin: trial pair=Sharp blunt: } \\
\text { Normal=Can distinguish sharp/not sharp }\end{array}$} \\
\hline \multirow[t]{4}{*}{ Achilles reflex } & Present $=0$ \\
\hline & $\begin{array}{l}\text { Present with } \\
\text { reinforcement }=1\end{array}$ \\
\hline & Absent $=2$ \\
\hline & NDS total out of 10 \\
\hline
\end{tabular}

NDS: Neuropathy disability score

\section{Reference}

1. Boulton AJ. Management of diabetic peripheral neuropathy.Clin Diabetes 2005;23:9-15. 
Annexure 5: Diabetic foot care ${ }^{[1]}$

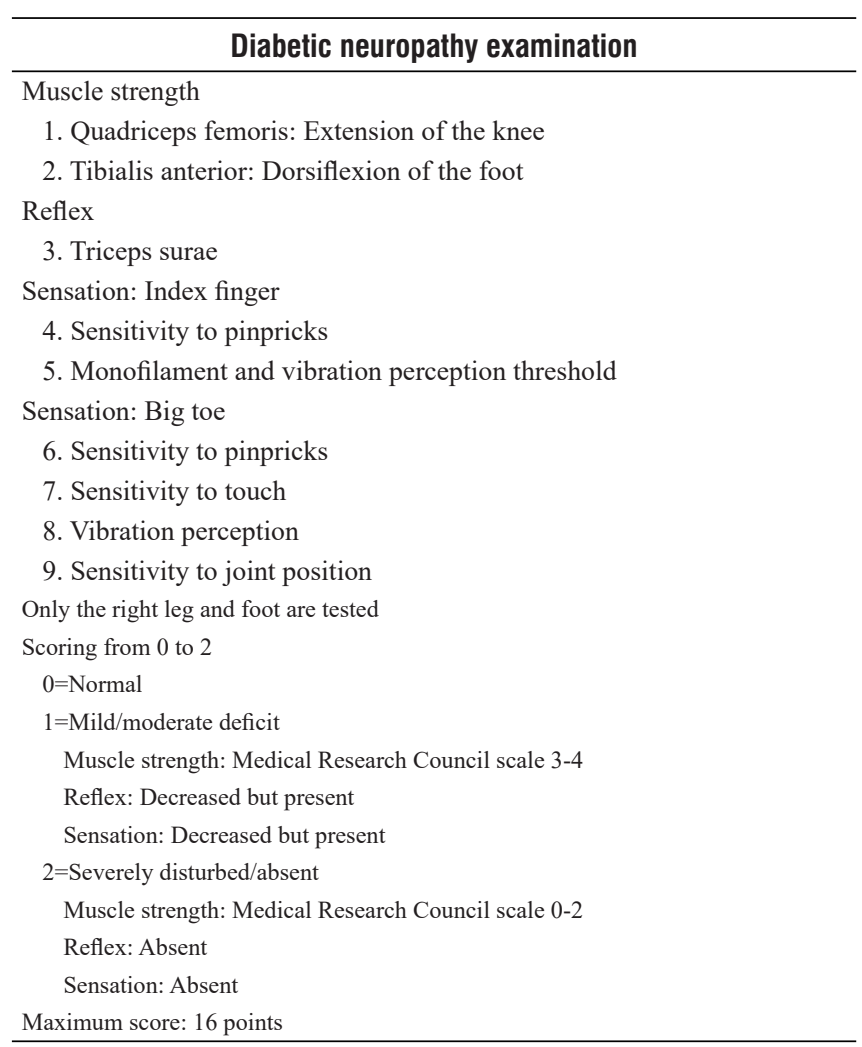

\section{REFERENCE}

1. Yang Z, Chen R, Zhang Y, Huang Y, Hong T, Sun F, Ji L, Zhan S. Scoring Systems to Screen for Diabetic Peripheral Neuropathy. Cochrane Database Syst Rev. 2014; 3: CD010974. DOI: 10.1002/14651858.CD010974.

\section{Diabetes and CV risk}

\section{Annexure 6: Statin treatment in patients with diabetes}

\begin{tabular}{lcl}
\hline Risk & Age (years) & Statins \\
\hline ASCVD risk & $<40$ & Moderate**/high intensity statins \\
factors* & $40-75$ & High intensity statins \\
& $>75$ & Moderate/high intensity statins \\
ASCVD & $<40$ & High intensity statins \\
& $40-75$ & High intensity statins \\
& $>75$ & High intensity statins \\
\hline *ASCVD ris facto
\end{tabular}

*ASCVD risk factors include LDL-C $\geq 100 \mathrm{mg} / \mathrm{dL}(2.6 \mathrm{mmol} / \mathrm{L})$, high

$\mathrm{BP}$, smoking, overweight and obesity, and family history of premature ASCVD, **Moderate intensity statins (OD): Atorvastatin 10-20 mg, rosuvastatin 5-10 mg, simvastatin $20-40 \mathrm{mg}$, pravastatin $40-80 \mathrm{mg}$, lovastatin $40 \mathrm{mg}$, fluvastatin XL $80 \mathrm{mg}$, pitavastatin 2-4 mg, ${ }^{\dagger} \mathrm{High}$ intensity statins (once daily): Atorvastatin 40-80 mg, rosuvastatin 20-40 mg. ASCVD: Atherosclerotic cardiovascular disease, BP: Blood pressure, LDL-C: Low-density lipoprotein-cholestrol, OD: Once daily 
Infections and Vaccinations

Annexure 7: Recommended vaccines for diabetic patients ${ }^{[1]}$

\begin{tabular}{|c|c|}
\hline Vaccines & Dosage \\
\hline $\begin{array}{l}\text { Pneumococcal } \\
\text { (polysaccharide) }\end{array}$ & 1 or 2 doses \\
\hline Influenza & 1 dose TIV annually \\
\hline $\begin{array}{l}\text { Tetanus diphtheria } \\
\text { pertussis (Td/Tdap) }\end{array}$ & $\begin{array}{l}\text { Substitute one time dose of Tdap; then boost with } \\
\text { Td every } 10 \text { years }\end{array}$ \\
\hline MMR & 1 or 2 doses, 4 weeks interval \\
\hline Varicella & 2 doses, at least 4 weeks apart \\
\hline Zoster & 1 dose, 60 years \\
\hline Hepatitis A & 2 doses at least 6 months apart \\
\hline Hepatitis B & 3 doses \\
\hline \multicolumn{2}{|l|}{ HPV } \\
\hline \multirow[t]{3}{*}{ Female } & 3 doses through age 26 years \\
\hline & $\begin{array}{l}\text { The second dose should be administered 1-2 } \\
\text { months after the first dose }\end{array}$ \\
\hline & $\begin{array}{l}\text { Third dose should be administered } 6 \text { months after } \\
\text { the first dose (at least } 24 \text { weeks after the first dose) }\end{array}$ \\
\hline Male & 3 doses through age 21 years \\
\hline
\end{tabular}

\section{REFERENCE}

1. Kesavadev J, Misra A, Das AK, Saboo B, Basu D, Thomas N, et al. Suggested use of vaccines in diabetes. Indian J Endocrinol Metab 2012;16:886-93.

\section{Fasting and Diabetes}

Annexure 8: Categories of risk in patients with T1DM or T2DM who fast during Ramadan

\begin{tabular}{|c|c|c|c|c|}
\hline Category & Parameter & I [very high risk] & II [high risk] & III [low/moderate risk] \\
\hline \multirow[t]{3}{*}{$\begin{array}{l}\text { Personal } \\
\text { characteristics }\end{array}$} & Life stage & $\begin{array}{l}\text { Childhood/adolescence/pregnancy/ } \\
\text { lactation/elderly }\end{array}$ & Late mid age & Healthy adulthood \\
\hline & Life style & $\begin{array}{l}\text { Intense physical labour; potential } \\
\text { public health impact of hypoglycaemia, } \\
\text { e.g., in commercial drivers }\end{array}$ & Variable duties, e.g., shiftworkers & Routine life style \\
\hline & Overall health & $\begin{array}{l}\text { Infirm; cognitive dysfunction; severe } \\
\text { acute illness }\end{array}$ & $\begin{array}{l}\text { Risk of dehydration; on } \\
\text { concomitant steroid therapy }\end{array}$ & Stable \\
\hline \multirow[t]{3}{*}{$\begin{array}{l}\text { Diabetes related } \\
\text { characteristics }\end{array}$} & Type of diabetes & $\begin{array}{l}\text { Brittle diabetes; T1DM, poorly } \\
\text { controlled }\end{array}$ & $\begin{array}{l}\text { T2DM, poorly controlled } \\
\text { T1DM, well controlled }\end{array}$ & T2DM, well controlled \\
\hline & Acute complications & $\begin{array}{l}\text { History of severe hypoglycaemia/ } \\
\text { DKA/HHNKC within } 3 \text { months prior } \\
\text { to Ramadan; history of recurrent } \\
\text { hypoglycaemia }\end{array}$ & None & None \\
\hline & $\begin{array}{l}\text { Chronic } \\
\text { complications }\end{array}$ & $\begin{array}{l}\text { History of hypoglycaemia } \\
\text { unawareness; CKD stage } 4 / 5 \text {; } \\
\text { advanced macrovascular }\end{array}$ & $\begin{array}{l}\text { CKD stage } 3 \text {, stable macrovascular } \\
\text { complications }\end{array}$ & No complication \\
\hline \multirow{2}{*}{$\begin{array}{l}\text { Therapeutic } \\
\text { characteristics }\end{array}$} & Noninsulin therapy & Conventional sulfonylurea & TID regimes & All other therapy \\
\hline & Insulin therapy & Basal bolus regimes & $\begin{array}{l}\text { TID regimes: Basal-plus; premixed } \\
\text { TDS; rapid-rapid-premix; } \\
\text { premix-rapid-premix }\end{array}$ & $\begin{array}{l}\text { Once or BID regimes: } \\
\text { Basal; premixed } \\
\text { analogues }\end{array}$ \\
\hline \multirow{2}{*}{$\begin{array}{l}\text { Medico-religious } \\
\text { advice }\end{array}$} & Religious suggestion & \multicolumn{3}{|c|}{ Listen to medical advice. Do not fast in health is endangered. Be prepared to break the fast if ill health occurs } \\
\hline & $\begin{array}{l}\text { Medical } \\
\text { management }\end{array}$ & \multicolumn{3}{|c|}{ Structured education; SMBG; Dose titration. Watch for complications, and manage appropriately } \\
\hline
\end{tabular}

T1DM: Type 1 diabetes, T2DM: Type 2 diabetes, DKA: diabetes ketoacidosis, HHNKC: Hyperosmolar hyperglycaemic nonketotic coma, CKD: Chronic kidney disease, TID: Thrice daily 


\section{Annexure 9: Dose adjustment/modifications for the management of T2DM during Ramadan fast ${ }^{[1-4]}$}

\begin{tabular}{|c|c|c|}
\hline Anti-diabetic agents & Current regimen & Recommended dose modification during Ramadan \\
\hline \multirow[t]{3}{*}{ Metformin } & OD & Take at iftar \\
\hline & BID & Take at iftar and suhur \\
\hline & TID & Take $2 / 3$ of the total daily dose at the iftar and the other $1 / 3$ at the suhur \\
\hline \multirow{2}{*}{ Sulfonylureas* } & OD & Take at iftar \\
\hline & BID & Take $1 / 2$ of usual evening dose with the suhur and the usual morning dose with the Iftar \\
\hline Glinides & \multicolumn{2}{|c|}{ The daily dose may be $\downarrow$ or divided to 2 doses according to meal size and should be taken at iftar and suhur } \\
\hline TZD & \multicolumn{2}{|c|}{ No dose adjustments is required } \\
\hline DPP-4 inhibitors & \multicolumn{2}{|c|}{ No dose adjustments required } \\
\hline Acarbose & \multicolumn{2}{|c|}{ No dose adjustments is required } \\
\hline SGLT-2 inhibitors ${ }^{\dagger}$ & \multicolumn{2}{|c|}{ No dose adjustment is required and the dose be taken with iftar } \\
\hline GLP-1 receptor agonists & \multicolumn{2}{|c|}{ The dose should be titrated 6 weeks prior to Ramadan and no dose adjustment is required } \\
\hline AGIs & \multicolumn{2}{|c|}{ No dose modification is required } \\
\hline \multirow[t]{2}{*}{ Long acting insulin } & OD & $\downarrow$ Dose by $15 \%-30 \%$ and take at iftar \\
\hline & BID & Take usual morning dose at iftar $\downarrow$ Evening dose by $50 \%$ and take at suhur \\
\hline \multirow[t]{2}{*}{ Short acting insulin } & & Take normal dose at iftar andlunch-time dose at dinner \\
\hline & & $\downarrow$ Suhur dose by $50 \%$ \\
\hline \multirow[t]{4}{*}{ Premixed insulin } & OD & Take normal dose at iftar \\
\hline & BID & Take $1 / 2$ of usual evening dose with the suhur and the usual morning dose with the Iftar \\
\hline & TID & Omit afternoon dose and adjust iftar and suhur doses \\
\hline & & Carry out dose titration every 3 days \\
\hline \multirow[t]{3}{*}{ Insulin pump } & Basal rate & $\downarrow$ Dose by $20 \%-40 \%$ in the last $3-4 \mathrm{~h}$ of fasting \\
\hline & & $\uparrow$ Dose by $0 \%-30 \%$ early after iftar \\
\hline & Bolus rate & Normal carbohydrate counting and insulin sensitivity principles apply \\
\hline
\end{tabular}

*Gliclazide and glimepiride should be preferred among all other sulphonylureas, ${ }^{\dagger}$ Elderly patients, patients with renal impairment, hypotensive individuals, those at risk of dehydration or those taking diuretics should not be treated with SGLT2 inhibitors. BID: Twice daily, TID: Thrice daily, OD: Once daily, AGIs: Alpha glucosidase inhibitors, DPP-4: Dipeptidyl peptidase-4, SGLT-2: Sodium-glucose co-transporter-2, TZD: Thiazolidinedione, GLP-1: Glucagon-like peptide 1

\section{ReFERENCES}

1. Kalra S, Aamir AH, Raza A, Das AK, Azad Khan AK, Shrestha D, et al. Place of sulfonylureas in the management of type 2 diabetes mellitus in South Asia: A consensus statement. Indian J Endocrinol Metab 2015;19:577-96.

2. Ibrahim M, Abu Al Magd M, Annabi FA, Assaad-Khalil S, Ba-Essa EM, Fahdil I, et al. Recommendations for management of diabetes during Ramadan: update 2015. BMJ Open Diabetes Res Care 2015;3:e00108.

3. Hassanein M, Al-Arouj M, Hamdy O, Bebakar WM, Jabbar A, Al-Madani A, et al. Diabetes and Ramadan: Practical guidelines. Diabetes Res Clin Pract 2017:126:303-16.

4. Al-Arouj M, Bouguerra R, Buse J, Hafez S, Hassanein M, Ibrahim MA, et al. Recommendations for management of diabetes during Ramadan. Diabetes Care 2005;28:2305-11. 